\title{
Health Disparities by Sexual Orientation Components in the United States
}

Julia Kay Wolf

West Virginia University, jkwolf@mix.wvu.edu

Follow this and additional works at: https://researchrepository.wvu.edu/etd

Part of the Demography, Population, and Ecology Commons, Gender and Sexuality Commons, and the Medicine and Health Commons

\section{Recommended Citation}

Wolf, Julia Kay, "Health Disparities by Sexual Orientation Components in the United States" (2021). Graduate Theses, Dissertations, and Problem Reports. 8132.

https://researchrepository.wvu.edu/etd/8132

This Dissertation is protected by copyright and/or related rights. It has been brought to you by the The Research Repository @ WVU with permission from the rights-holder(s). You are free to use this Dissertation in any way that is permitted by the copyright and related rights legislation that applies to your use. For other uses you must obtain permission from the rights-holder(s) directly, unless additional rights are indicated by a Creative Commons license in the record and/ or on the work itself. This Dissertation has been accepted for inclusion in WVU Graduate Theses, Dissertations, and Problem Reports collection by an authorized administrator of The Research Repository @ WVU.

For more information, please contact researchrepository@mail.wvu.edu. 
Health Disparities by Sexual Orientation Components in the United States

\author{
Julia Kay Wolf
}

Dissertation submitted

to the Eberly College of Arts and Sciences

at West Virginia University

in partial fulfillment of the requirements for the degree of

Doctor of Philosophy in

Sociology

\author{
Jeralynn S. Cossman, Ph.D., Co-Chair \\ Christopher P. Scheitle, Ph.D., Co-Chair \\ Christiaan G. Abildso, Ph.D. \\ Robert M. Bossarte, Ph.D. \\ Department of Sociology and Anthropology
}

Morgantown, West Virginia

2021

Keywords: fundamental causes, health disparities, minority stress, sexual minority health Copyright 2021 Julia Kay Wolf 


\begin{abstract}
Health Disparities by Sexual Orientation Components in the United States
\end{abstract}

Julia Kay Wolf

Fundamental cause theory encourages researchers to consider broad social conditions that put people at risk of individual-level health-related risks that can lead to health disparities between social groups. Stigma has recently been proposed as a fundamental cause of health disparities as it influences multiple disease outcomes, affects access to resources, and is consistently related to health inequities across historical and geographical contexts. Minority stress theory describes how sexual minorities endure excess stressors in the form of prejudice and discrimination due to their stigmatized status. Considering both frameworks, I explore how stigmatized sexual orientation minority respondents compare to their sexual majority counterparts on a holistic subjective measure of health, an objective measure of health, and a measure regarding access to health care. Sexual minority health research has grown rapidly in recent years, but data and methodology limitations have restricted analyses and subsequent topic knowledge at a national level. Using nine years of nationally representative data from the National Survey of Family Growth (NSFG; 2011-2019), I explore three health-related variables - self-rated health (SRH), body mass index (BMI), and access to a usual source of health care (USOC) - by three components of sexual orientation - sexual orientation identity, sexual attraction, and sexual behavior. I provide summary statistics on these elements and other demographic and socioeconomic factors as well as report logistic and multinomial regression results on the healthrelated variables by each sexual orientation component. Chapter one analyzes the entire NSFG sample except for respondents who were pregnant, under age 25, and/or were missing responses for any variables used $(\mathrm{N}=23,567)$. Chapter two splits the qualified sample into females $(\mathrm{N}=12,895)$ and males $(\mathrm{N}=10,672)$ and Chapter three splits the qualified sample into two age groups (25-34 years old, $\mathrm{N}=13,038 ; 35-44$ years old, $\mathrm{N}=10,529)$ - both report the results of the same analyses on the split samples. Only one result was consistently found across chaptersthose who have had sexual experiences with someone of the same sex have lower odds of reporting excellent, very good, or good SRH compared to those who have had no same-sex sexual experience. All other significant differences vary by group analyzed; the male sample had the highest number of significant differences in health outcomes by sexual orientation components and the 35-44 age group sample had the least number of significant differences. We must use an intersectional perspective that considers other statuses such as sex and age to properly investigate and address health-related issues for sexual orientation minorities. 


\section{TABLE OF CONTENTS}

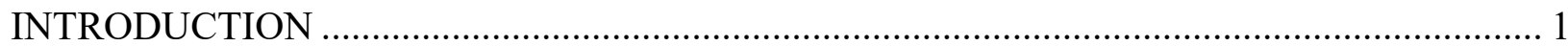

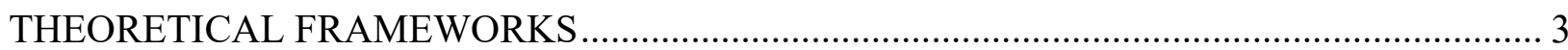

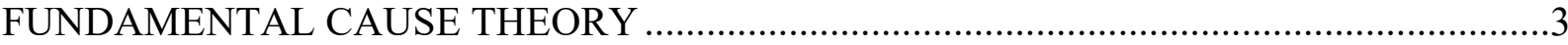

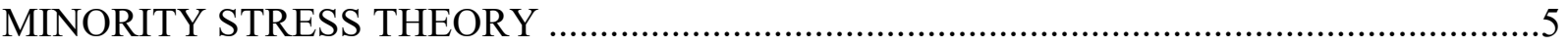

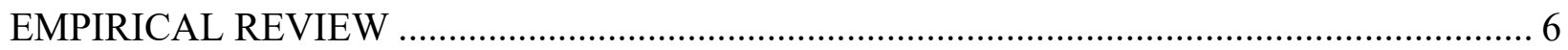

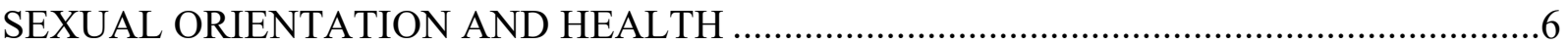

CONTRIBUTION TO THE LITERATURE ......................................................................... 9

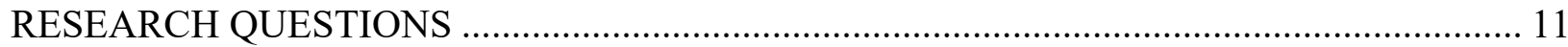

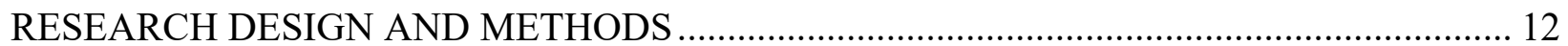

DATA

Dataset: National Survey of Family Growth (NSFG; 2011-2019) ...................................... 12

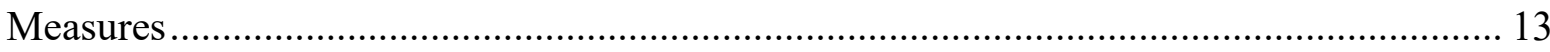

CHAPTER 1 - Health Disparities by Sexual Attraction, Behavior, and Identity in the United

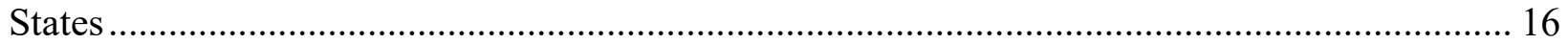

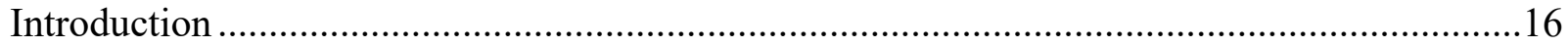

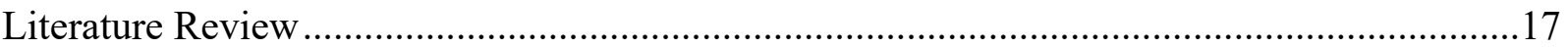

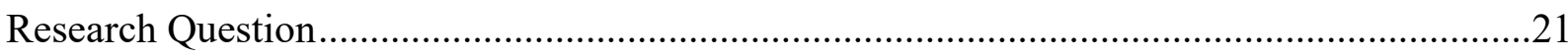

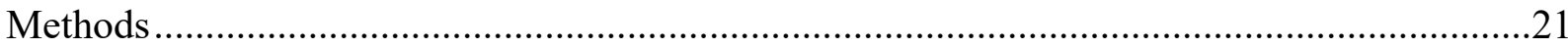

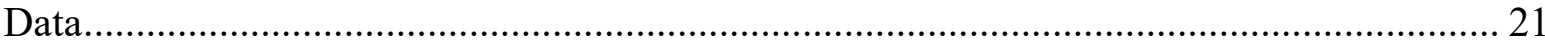

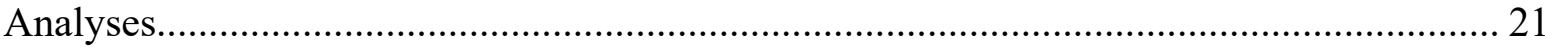

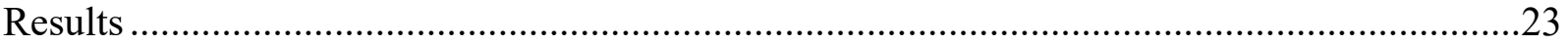

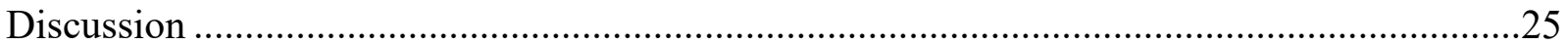

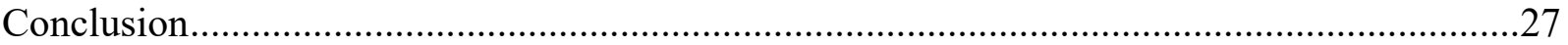


CHAPTER 2 - Health Disparities by Sexual Orientation Components among a Nationally Representative Sample of Males and Females in the United States ...................................... 28

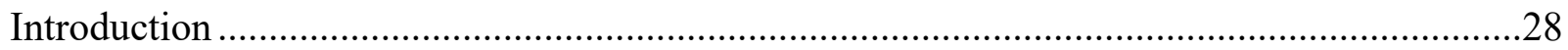

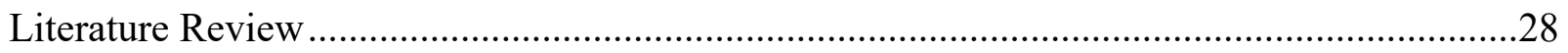

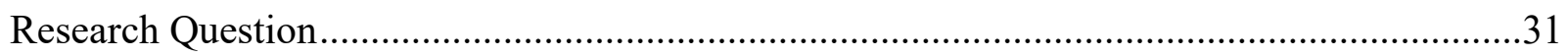

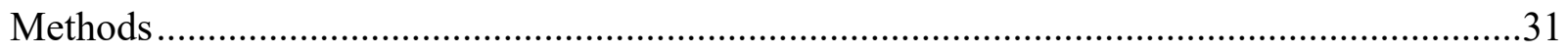

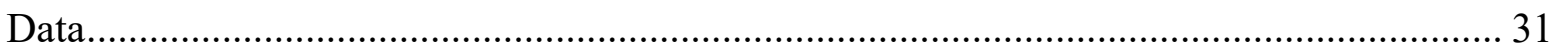

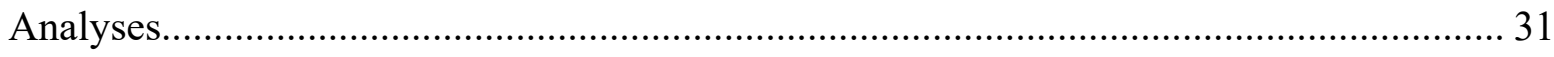

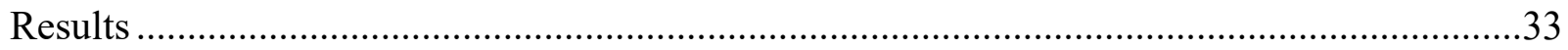

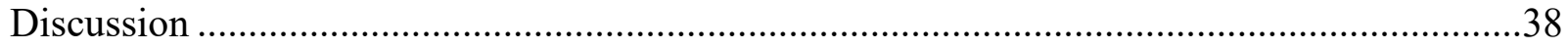

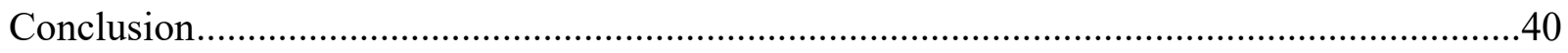

CHAPTER 3 - Exploring Health Disparities by Sexual Attraction, Behavior, and Identity by Age

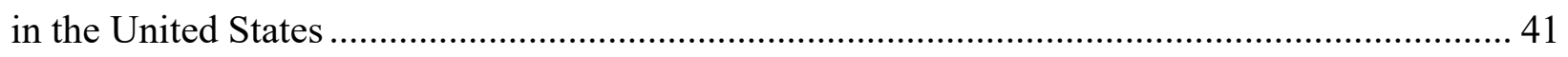

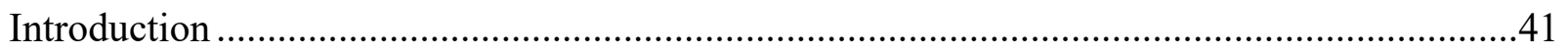

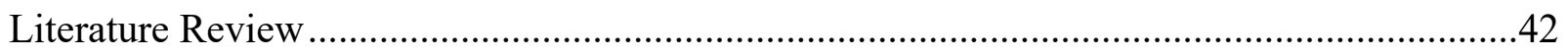

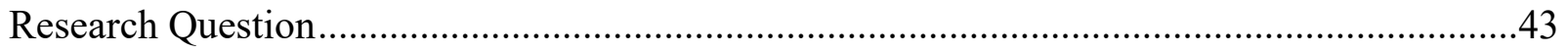

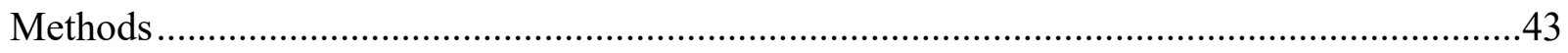

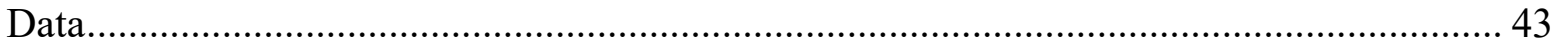

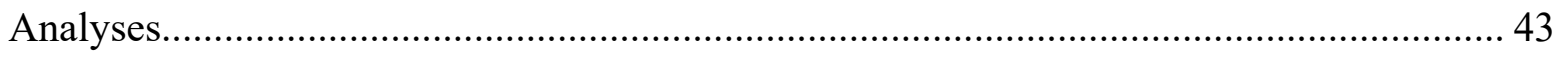

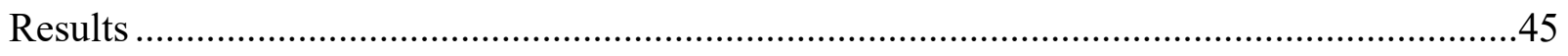

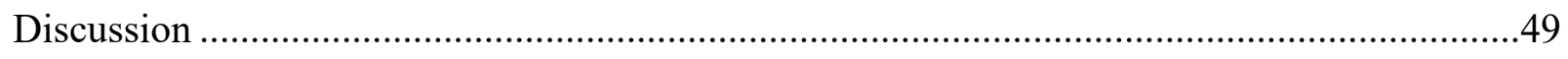

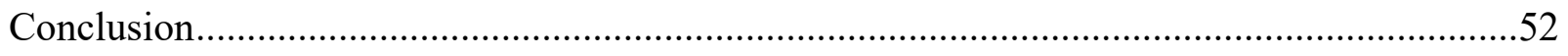

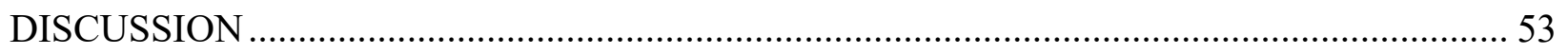

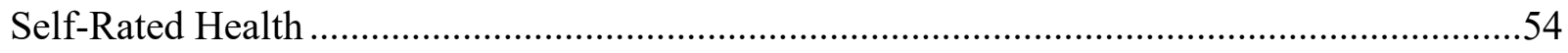

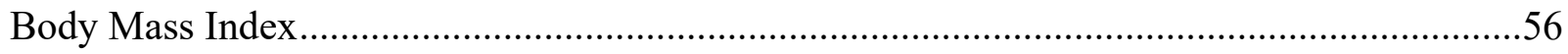

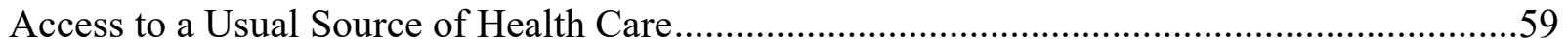




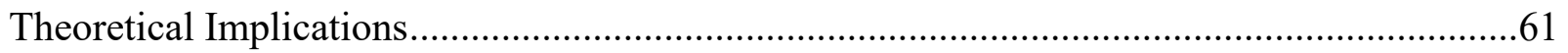

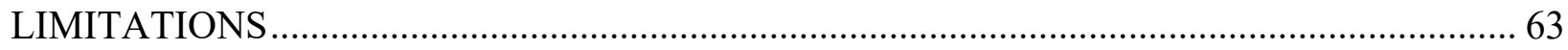

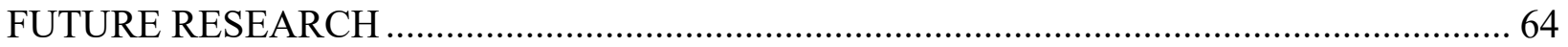

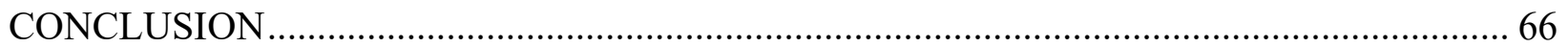

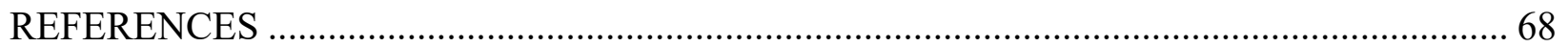

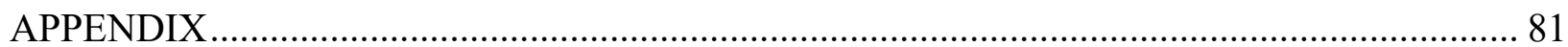




\section{LIST OF TABLES}

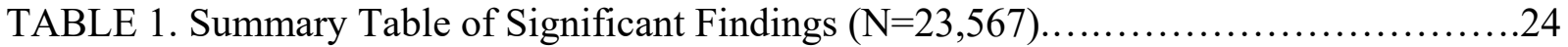

TABLE 2.1. Summary Table of Significant Findings from Female Sample $(\mathrm{N}=12,895) \ldots \ldots .37$

TABLE 2.2. Summary Table of Significant Findings from Male Sample $(N=10,672) \ldots \ldots \ldots . .37$

TABLE 3.1. Summary Table of Significant Findings from Sample Aged 25-34 (N=13,038)..49

TABLE 3.2. Summary Table of Significant Findings from Sample Aged 35-44 (N=10,529)..49

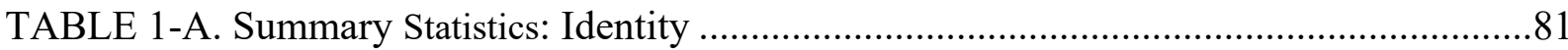

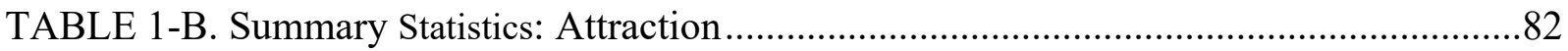

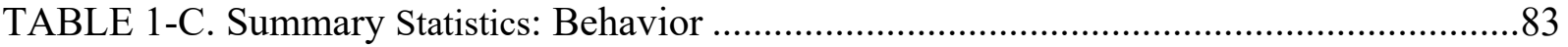

TABLE 1-D. Summary Statistics: Components and Outcomes..................................................84

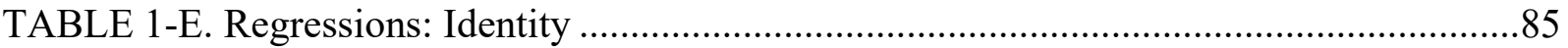

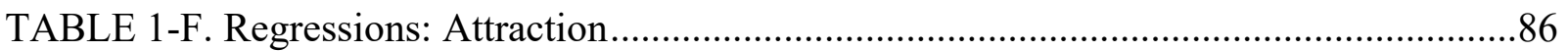

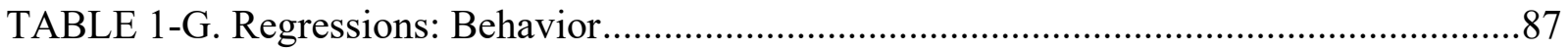

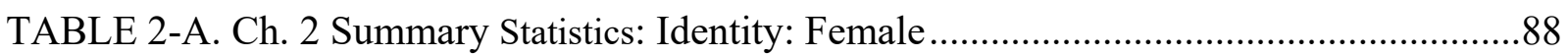

TABLE 2-B. Ch. 2 Summary Statistics: Attraction: Female ……………………………..........89

TABLE 2-C. Ch. 2 Summary Statistics: Behavior: Female ………………………………….....90

TABLE 2-D. Ch. 2 Summary Statistics: Components and Outcomes: Female ...........................91

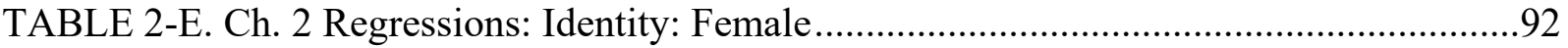

TABLE 2-F. Ch. 2 Regressions: Attraction: Female ……………………………………......93

TABLE 2-G. Ch. 2 Regressions: Behavior: Female …………................................................

TABLE 2-H. Ch. 2 Summary Statistics: Identity: Male …………………………………........95

TABLE 2-I. Ch. 2 Summary Statistics: Attraction: Male ………………………………….......96

TABLE 2-J. Ch. 2 Summary Statistics: Behavior: Male...............................................................97

TABLE 2-K. Ch. 2 Summary Statistics: Components and Outcomes: Male.................................98

TABLE 2-L. Ch. 2 Regressions: Identity: Male ………………...............................................99 


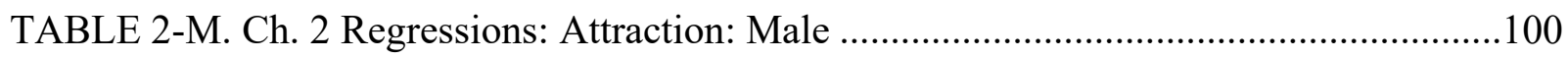

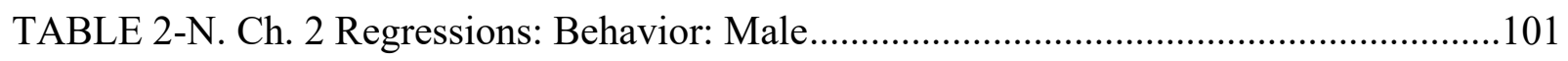

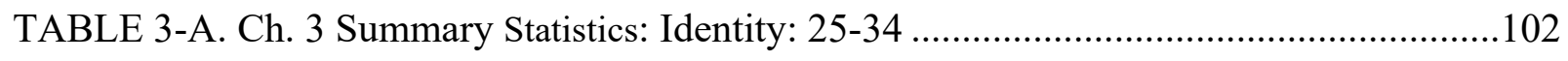

TABLE 3-B. Ch. 3 Summary Statistics: Attraction: 25-34 .............................................103

TABLE 3-C. Ch. 3 Summary Statistics: Behavior: 25-34 „................................................104

TABLE 3-D. Ch. 3 Summary Statistics: Components and Outcomes: 25-34_......................105

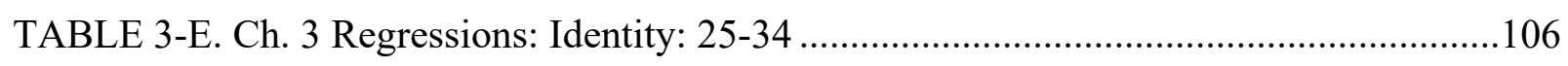

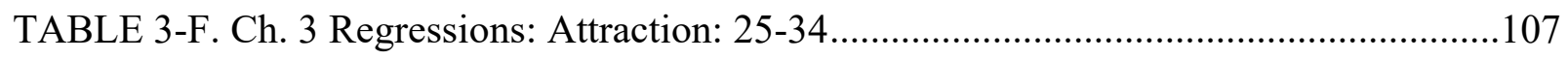

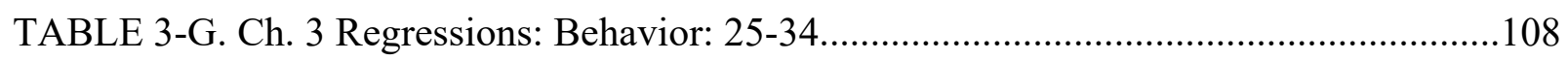

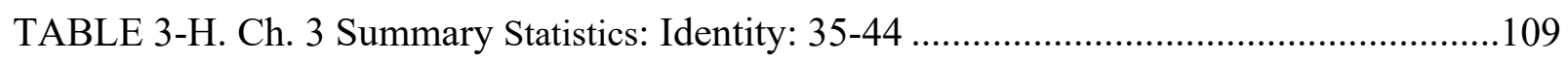

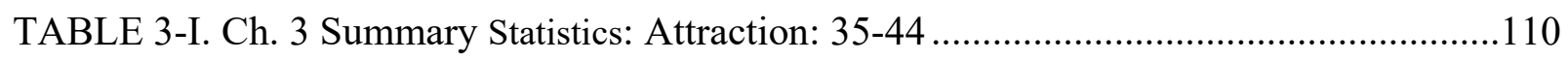

TABLE 3-J. Ch. 3 Summary Statistics: Behavior: 35-44 ...............................................111

TABLE 3-K. Ch. 3 Summary Statistics: Components and Outcomes: $35-44 \ldots \ldots \ldots \ldots \ldots \ldots \ldots \ldots \ldots . . . . . . . .112$

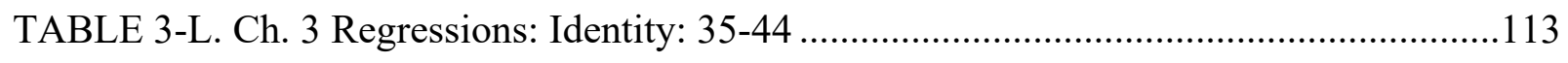

TABLE 3-M. Ch. 3 Regressions: Attraction: 35-44 …....................................................114

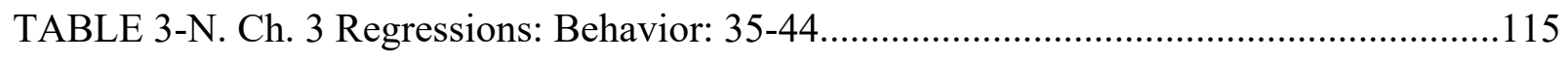




\section{LIST OF ABBREVIATIONS}

Body mass index (BMI)

Confidence interval (CI)

Metropolitan statistical area (MSA)

Multinomial logistic regression (MLR)

National Survey of Family Growth (NSFG)

Odds ratio (OR)

Relative risk ratio (RRR)

Self-rated health (SRH)

Usual source of (health) care (USOC) 


\section{INTRODUCTION}

Blatant health disparities by social group membership have existed across the United States for decades. Differences in socioeconomic status (SES) have been a basic and enduring social condition contributing to these disparities. As such, Link and Phelan (1995) argue that SES should be considered a fundamental cause of disease. A clear gradient can be found between SES (i.e., occupation, income, and education) and health — as SES increases, health behaviors and outcomes typically improve. While individual risk factors, such as smoking or physical activity levels, contribute to health disparities, SES influences these and other individual health behaviors by determining access to valuable resources such as "money, power, prestige, and/or social connectedness" that provide knowledge or tools for maximizing health (Link and Phelan 1995:87). Those without access to these resources are at an inequitable disadvantage. Minority group members are at risk of stigmatization which can result in lower SES and they may experience minority stress which can also negatively affect their health (Meyer 2003). While this dynamic has been often considered regarding social statuses like gender and race/ethnicity, which were also considered fundamental causes of disease by Link and Phelan (1995), it has only recently been considered regarding sexual orientation.

We can see disparities by sexual orientation across a variety of health behaviors and outcomes (Institute of Medicine 2011). These disparities are not unexpected, as those who are non-heterosexual individuals have been stigmatized in the United States. Minority stress theory posits that members of stigmatized groups are more likely to face daily stressors (e.g., microaggressions), prejudice, and discrimination that majority group members will not face; such additional stress can lead to mental and physical health problems (Meyer 2003). Akin to gender and racial/ethnic minorities, sexual orientation minorities also do not have the same level of access to the resources their group-majority counterparts have. For example, same-sex couples just earned the right to marry only five years ago in the United States (Anon 2015), meaning they were not privy to financial or social benefits that come with legal marriages. The US Supreme Court ruled that employers cannot discriminate based on sexual orientation just a few months ago (Rushe 2020). Previous studies had found evidence of workplace discrimination, including lower income for gay men compared to heterosexual men, though lesbians tended to earn more than heterosexual women but less than men (Badgett et al. 2009). However, research does suggest that "same-sex couples are more vulnerable to poverty in general than are different-sex 
couples" (Badgett, Durso, and Schneebaum 2013:1). Although there are mixed findings on whether or not sexual minorities tend to have higher educational attainment than their heterosexual or different-sex couple counterparts (Gates and Newport 2012; Hasenbush et al. 2014), they are still subject to more bullying in school (Kosciw et al. 2020). Thus, it makes sense to consider sexual orientation as a fundamental cause of disease as it affects access to each of the three elements that generally comprise SES.

It is a complex task to accurately measure those who are sexual orientation minorities and, subsequently, their health (Gates 2011). Sexual orientation is generally thought to consist of three components: attraction, behavior, and identity (Igartua et al. 2009). However, these components do not necessarily align for everyone. For example, one may be attracted to males/men and females/women, but to fit into society's idea of appropriate sexual behavior and identity, they may refrain from acting on their attractions to the same-sex/gender and commit to a heterosexual identity. Additionally, one's identity may be fluid and change over time (Scheitle and Wolf 2018), particularly as people converse about experiences and new identities emerge with definitions that may more closely align with one's feelings. Further, those who identify as a sexual orientation minority, but are able to "pass" as heterosexual in public or in other dangerous situations may be able to avoid overt discrimination or ridicule, though such concealment of one's identity could negatively affect their well-being too (Funders for Lesbian and Gay Issues 2004; Riggle et al. 2017). Studies that have considered all three components have found health differences amongst (e.g., between attraction and behavior) and within (e.g., between gays/lesbians and bisexuals) them (Wolff et al. 2017). Therefore, considering multiple measures of sexual orientation are important for understanding health disparities.

In addition to considering three components of sexual orientation, comprehensive studies on sexual minority health need to examine the influences of other social statuses contributing to health disparities. Using an intersectional perspective allows researchers to see how multiple identity affiliations affect health behaviors and outcomes. For example, differences in health outcomes vary by both sexual orientation and gender/sex as sexual minority women may face double (or more) stigmatization from being in at least two minority groups (Szymanski 2005). Health disparities may also exist by age groups as different life course milestones are reached or expected. Although a few studies have explored differences in sexual orientation by age (e.g., Mosher, Chandra, and Jones 2005; Savin-Williams and Vrangalova 2013), research is needed to 
study potential differences in regards to age and sexual minority health.

These dissertation chapters investigate each of the three components of sexual orientation - attraction, behavior, and identity — and their associations with self-rated health (SRH), body mass index (BMI), and access to a usual source of health care (USOC). Chapter 1 explores these relationships broadly while Chapter 2 disentangles differences by sex and Chapter 3 examines the differences between two age groups. I use the National Survey of Family Growth (NSFG) surveys from 2011-2013, 2013-2015, 2015-2017, and 2017-2019 to complete all analyses. The following sections discuss the theoretical frameworks employed throughout each chapter, a general overview of the empirical research on sexual minority health, how my research contributes to the literature, and my research questions. The subsequent section covers the NSFG datasets and variables I use since all three chapters use the same data. Each chapter is designed as an article with an introduction, specific literature review, recap of the data, analyses, results, discussion, and conclusion. An overall discussion section follows all three chapters as well as limitations, future research, and a final conclusion. Tables are provided in the appendix after the reference section.

\section{THEORETICAL FRAMEWORKS}

\section{FUNDAMENTAL CAUSE THEORY}

Fundamental cause theory was proposed to explain why group-level disparities in disease can be found throughout history, despite advancements in health care, medication, technology, and public sanitation. While general trends show improvements in health over time (e.g., longer life expectancies, eradications of infectious diseases like polio), there are still discernable gaps between groups of people with some benefiting greatly and others at higher risk of disease and death. Link and Phelan (1995) offer an explanation for these still present variations in health outcomes: some social factors are so crucial to accessing resources to prevent or mitigate adverse health outcomes that they should be considered fundamental causes of disease.

At the time of Link and Phelan's (1995) writing, epidemiological research had been focusing on individual-level factors that caused disease such as diet, cholesterol, and exercise. While noteworthy in making these connections, Link and Phelan (1995) suggest that they are too fixated on proximal (i.e., immediate) causes of disease and are missing the larger picture of how people become at risk of these risk factors. That is, they are ignoring the social conditions or contexts that are putting groups of people at risk of poor diets, high cholesterol, and inadequate 
exercise in the first place. The proximal individual-level factors are considered to be "downstream" in relation to disease causation while fundamental cause theory is meant to encourage an "upstream" approach to see what distal social factors are driving differences in individual-level factors.

Link and Phelan (1995) consider socioeconomic status (SES) to be a fundamental cause of disease. They came to this conclusion by first addressing the research pertaining to the causal direction between these two variables and saying it demonstrates that SES has a greater effect on disease (i.e., social causation) rather than disease affecting SES (i.e., social selection), though both undoubtedly happen. Second, they point out that SES continues to affect health outcomes even if intervening mechanisms (i.e., proximal individual-level factors) are addressed. They note that new intervening mechanisms will just continue to take the place of old ones, keeping the connection between SES and disease constant. For example, they note that advancements in sanitation and immunization helped improve health, but then other risk factors like smoking and exercise took their place to link SES and disease, thus leading to continuing disparities in health.

In explaining why SES is such a powerful driver of health outcomes, Link and Phelan (1995) highlight its tie to accessing resources. Those with high SES have better access to resources such as "money, knowledge, power, prestige, and the kinds of interpersonal resources embodied in the concepts of social support and social network" (Link and Phelan 1995:87). Access to these assets allow people to take steps to minimize their exposures to risk factors or consequences of any exposure and therefore remain healthier than those who do not have access to the same resources. With an unequal distribution of resources, particularly in the United States, health disparities will continue to exist.

Other social conditions can be considered fundamental causes of disease or health disparities. Link and Phelan (1995) briefly mention gender, race/ethnicity, social support, social networks, and stigmatization as "potential fundamental causes" due to their close relationship with access to resources (87). Gender minorities, racial/ethnic minorities, and other stigmatized groups live in a society that tends to cater towards those in power-majority group members. Thus, minority group members are often restricted in their access to knowledge, tools, or other people that can help improve their health. Minority group members also face additional stressors - minority stress - that can negatively affect their health. Hatzenbuehler, Phelan, and Link (2013) recently mentioned another status where minority group members are often 
stigmatized resulting in less access to resources and poorer health-sexual orientation. MINORITY STRESS THEORY

Stress can lead to poor health behaviors (e.g., coping with stress by drinking alcohol or using illicit drugs) and adverse health outcomes (e.g., psychological problems and physical illness) (Thoits 1995). However, stress is not always distributed randomly across a population (Aneshensel 1992). Systemic stressors - stressors that are tied to one's social status or group membership — present a clear threat to some people and not to others (Aneshensel 1992). In the US, members of minority social groups face systemic stressors due to prejudice and discrimination (e.g., sexism, racism, and ableism) that members of the majority social groups do not have to endure.

Minority stress theory conceptualizes the process whereby members of a stigmatized minority group experience prejudice and discrimination which "create a stressful social environment" and consequent mental health problems (Meyer 2003:674-675). Lesbian, gay, bisexual, queer/questioning individuals, and those who use sexual orientation identities that are not heterosexual (LGBQ+) are members of the sexual orientation minority group. LGBQ+ individuals can be subject to a variety of mistreatments broadly stemming from homophobia 1 "the fear and hatred of homosexuality or perceived homosexuality" — and heterosexism — "the belief that heterosexuality is the best and only acceptable way of living" (Elia 1993:178). Minority stress theory states that in addition to the daily stressors and stressful life events that humans face, minority group members experience these and other types of stress due to their stigmatized status.

Meyer (2003) emphasizes the additional stress that they face is multifaceted: ...minority stress is (a) unique - that is, minority stress is additive to general stressors that are experienced by all people, and therefore, stigmatized people are required an adaptation effort above that required of similar others who are not stigmatized; (b) chronic - that is, minority stress is related to relatively stable underlying social and cultural structures; and (c) socially based - that is, it stems from social processes, institutions, and structures beyond the individual rather than individual events or conditions that characterize general stressors or biological, genetic, or other nonsocial characteristics of the person or group. (P. 676)

Minority stress theory is also constructed on the assumption of social causation (Meyer 2003). Link and Phelan (1995) found more support for social causation over social selection for

\footnotetext{
${ }^{1}$ Phobias for specific identities such as biphobia against bisexuals and aphobia against asexuals also exist.
} 
fundamental cause theory, and similarly Lick, Durso, and Johnson (2013) found relevant literature supporting social causation over social selection in regards to minority stress and physical health among sexual minorities. In other words, minority stress tends to more often lead to poorer health rather than poorer health leading to minority stress.

There are a variety of different stressors that sexual minorities may face. Stressors, can appear in the form of "traumatic events, eventful life stressors, chronic stress, and roles strains, as well as daily hassles and even nonevents as varied components of stress" that can lead to adverse health outcomes (Meyer 2003:675). For example, sexual minorities report a higher prevalence of stressful childhood experiences than their heterosexual counterparts (Schneeberger et al. 2014), experience bullying in school (Kosciw et al. 2020), and face discrimination in the workplace (Badgett et al. 2009). Sexual orientation itself is not what leads to more adverse health outcomes, but rather the stress, discrimination, and victimization faced by sexual minorities due to a stigmatized identity that leads to it.

Stress can be "taxing to individuals and exceed their capacity to endure, therefore having potential to induce mental or somatic illness" (Meyer 2003:675). Thus, it follows that LGBQ+ individuals would have higher rates of mental or somatic illnesses than their sexual majority counterparts who do not experience additional minority stress. Sexual orientation minorities have been documented to experience higher levels of stress and, consequently, higher levels of negative health behaviors and outcomes than their heterosexual counterparts as discussed in the following section. Although Meyer (2003) focused on mental health outcomes, he briefly mentions physical health ailments influenced by minority stress. Recent work has found support for applying the framework to physical health outcomes among sexual minorities (Strutz, Herring, and Halpern 2015). My dissertation focuses on physical health (i.e., BMI) and a holistic subjective measure of health (i.e., SRH) in an attempt to bolster this lesser studied part of the literature.

\section{EMPIRICAL REVIEW}

\section{SEXUAL ORIENTATION AND HEALTH}

Ample research examining sexual orientation minorities, particularly among those who are lesbian, gay, or bisexual (LGB) have found relationships between their sexual orientation identities and poorer health compared to their heterosexual counterparts (Boehmer, Bowen, and Bauer 2007; Bowen, Balsam, and Ender 2008; Diamant et al. 2000; Fish and Pasley 2015; 
Hatzenbuehler 2009; Kelleher 2009; Lick et al. 2013; Meyer 2003; Schneeberger et al. 2014; Ward et al. 2014). These disparities can be found across the life course for sexual minorities from higher rates of depression and suicide among sexual minority youth (Hafeez et al. 2017; Marshal et al. 2011) to social isolation among sexual minority elderly (Fredriksen-Goldsen, Kim, et al. 2013; Grant 2010). LGB adults report more lifetime and daily experiences of discrimination than their heterosexual counterparts (Mays and Cochran 2001), are more likely to be current smokers (Blosnich et al. 2014), and research shows "elevated risks for depression, anxiety, suicide attempts or suicides, and substance-related problems for SM [sexual minority] men and women" (Plöderl and Tremblay 2015:367). However, sexual minorities are not a homogeneous group. It is important to investigate differences within sexual minority groups too.

Recent research has been able to analyze health outcomes between sexual orientation identities beyond just that of "heterosexual" vs. "LGB" and found variations across groups (Blosnich et al. 2014; Boehmer et al. 2007; Bowen et al. 2008; Chaudhry and Reisner 2019; Fish 2019). For example, bisexual adults are more likely to experience major depressive episodes and substance abuse or dependence than other sexual minority adults or heterosexual adults (Chaudhry and Reisner 2019). Bisexuals tend to be at greater risk of poor health behaviors and outcomes than heterosexuals and even their gay and lesbian counterparts (Gorman et al. 2015). They are at risk of experiencing biphobia - "negative attitudes about bisexuality and bisexual individuals" (Bennett 1992; as cited by Mulick and Wright, Jr. 2002:47)—from both heterosexuals for not being straight and from lesbians and gay men for not being gay enough. However, evidence of their greater health risks is somewhat equivocal, particularly when explored by gender or sex (Boehmer et al. 2007; Lee, Griffin, and Melvin 2009).

Differences exist between males and females as well as between sexual orientation identities. Dilley and colleagues (2010) found both sexual minority men and women to be more likely to smoke and to have poorer mental health than their heterosexual counterparts, but only sexual minority women were more likely to drink excess alcohol, have asthma and poorer physical health, and be overweight while sexual minority men were more likely to have healthlimited activities than their heterosexual counterparts. Blosnich and colleagues (2014) found a variety of differences in health-related outcomes depending on the sexual minority group stratified by gender (i.e., lesbian women, bisexual women, gay men, bisexual men) in question. Sexual minority men and women differ in their perceptions on body image as well which can 
affect how they eat, exercise, or view themselves (Herrick and Duncan 2018). In their paper examining sexual orientation and gender in regards to health, Gorman and colleagues (2015) advocate for an intersectional perspective as they contend it has "developed separately from dominant paradigms in health research, including fundamental cause theory and social ecology" (1379). Gender differences in health-related outcomes by sexual orientation cannot be ignored.

Due to the nature of the population in question, many of these studies regarding sexual minorities suffer from data and methodological limitations. Simply defining who is considered a sexual minority is a complex feat (Gates 2011). There are different ways to word questions when asking about sexual orientation, and those constructing surveys need to consider their target population age and race/ethnicity because they can differ in their understandings of terms (Durso and Gates 2013). For example, older adults may not know what the terms "gay" or "heterosexual" mean and the term "two-spirit" has typically been used within Native American culture (Durso and Gates 2013). Sexual orientation identity questions may also provide response options such as "something else," "don't know," "refused," "unsure," or "other" and respondents selecting them have typically been dropped from analyses (Barnes et al. 2014; Blosnich and Bossarte 2012; Chaudhry and Reisner 2019; Scheitle and Wolf 2018). Sexual orientation identity is also only one of three components of sexual orientation. Sexuality research finds that attraction and behavior are two more components of sexual orientation that should be considered (Gates 2011; Institute of Medicine 2011). There are differences across surveys and even inconsistencies in question construction of individual surveys over time (Gates 2011).

Problems with sampling are also prevalent in sexual minority literature. Meyer (2003) points out issues such as using a convenience (non-probability) sample, splitting samples into heterosexual vs. sexual minority as opposed to exploring sexual identities separately, and measuring sexual minority status using one variable despite sexual orientation being composed of three components. Studies using convenience samples (e.g., Bird, Kuhns, and Garofalo 2012; Frost, Meyer, and Schwartz 2016; Mahaffy 1996; McGregor et al. 2001) are less generalizable to the population as a whole and may consist of respondents who are biasedly more open about their sexual orientation and/or mental health. Splitting samples into heterosexual vs. sexual minority (e.g., Barnes et al. 2014; Boehmer et al. 2007; Bostwick, Hughes, and Everett 2015; Garofalo et al. 1998; Mays and Cochran 2001) inaccurately assumes homogeneity within the groups being compared (Institute of Medicine 2011). Measuring sexual orientation by identity 
ignores exploring how attraction or behavior plays a role in health outcomes. Some probability based samples are limited geographically (e.g., Blosnich et al. 2014 used population-based data from only ten US states and none were from the South) and cannot be generalized to different contexts. Healthy People 2020 (2020) notes that only six national data systems collected sexual orientation data prior to 2010 and just two more were added in 2013 and 2015. While there are studies that have been able to overcome some of these limitations (e.g., Blosnich and Bossarte 2012; Chaudhry and Reisner 2019; Everett 2015), more data and research is needed to comprehensively understand sexual minority health disparities across the United States.

\section{CONTRIBUTION TO THE LITERATURE}

My dissertation continues the synthesis of the sexual orientation-health literature with the fundamental cause framework as recommended by Hatzenbuehler, Phelan, and Link (2013). Those who identify as a sexual orientation minority are at greater risk of multiple adverse health behaviors and outcomes than their heterosexual counterparts. Research shows lower access to health-related resources for sexual orientation minorities and particular laws in the US, until recently, explicitly denied them access to resources such as financial stability of marriage and employment without discrimination. Sexual orientation is still not included as a protected status in the 1964 Civil Rights Act that prohibits discrimination against them in various public arenas (Stracqualursi 2021). Stigma has also inhibited access to social support such as the tangible and intangible benefits of an accepting family or tolerant school environment. Being a sexual orientation minority puts one at risk of risks that can negatively affect their mental and physical health.

One study has already acknowledged this hypothesis and tested it. Bränström and colleagues (2016) investigate whether or not fundamental cause theory can explain health disparities by sexual orientation. By exploring differences in several health outcome variables ranked by preventability, they found "an increased prevalence of high-preventable diseases among sexual minority individuals [those who identified as homosexual or bisexual] as compared with heterosexuals and no sexual orientation identity differences with respect to lowpreventable diseases" (Bränström et al. 2016:1113). Since fundamental cause theory posits that disease disparities arise between groups with inequitable access to resources to prevent or mitigate disease, these findings support fundamental cause theory as access to a greater amount of resources still cannot help with regards to low-preventable diseases where "causes and 
cures...are unknown" (Phelan, Link, and Tehranifar 2010:S31). Finding no differences between the heterosexual and homosexual/bisexual groups for low-preventable diseases while finding differences for high-preventable diseases suggests unequal access to important health-related resources.

Another relevant paper suggests that stigma should be considered a fundamental cause of disease disparities at the population level (Hatzenbuehler et al. 2013). This idea gets at the root reason of why sexual orientation status (i.e., a stigmatized status) can be considered a fundamental cause of health disparities in the United States. Minority stress theory also aligns closely with this notion as it too points out how stigmatized minority statuses endure greater stressors leading to poor health behaviors and outcomes. The authors of fundamental cause theory suggest stigma as a fundamental cause of population health disparities because of its broad scope that then encompasses other stigmatized statuses such as race/ethnicity, disability, and sexual orientation (Hatzenbuehler et al. 2013).

To be considered a fundamental cause of disease, a social condition must 1) affect multiple health/disease outcomes via multiple pathways; 2) affect access to resources that can be used towards health improvement/maintenance and/or disease treatment; and 3) be consistently related to such health disparities over time and in different contexts (Hatzenbuehler et al. 2013; Link and Phelan 1995). This dissertation contributes to the literature by examining two healthrelated outcomes and access to a health-related resource via a nationally representative sample of the United States over a nine-year period by sexual orientation groups based on attraction, behavior, and identity. It also explores how sex and age play a role. In particular, the following chapters explore how self-rated health (SRH), BMI, and access to a usual source of health care, vary by: 1) sexual orientation component; 2) sexual orientation component and sex; and 3) sexual orientation component and age group.

Self-rated health is "one of the most frequently employed health indicators in sociological health research since the 1950s" (Jylhä 2009:307). Although it is a subjective measure, it has been found to be highly predictive of mortality (Schnittker and Bacak 2014; Stoddard et al. 2019) and disability (Farmer and Ferraro 1997; Ferraro, Farmer, and Wybraniec 1997; Ferraro and $\mathrm{Su}$ 2000; Idler and Kasl 1995). It has also been found to be influenced by perceived racial discrimination (Chen and Yang 2014), so this dissertation is a first step towards considering how another stigmatized group might report SRH. Previous research on young adult sexual minority 
health by sexual orientation component has used this measure dichotomously as I use here (Strutz et al. 2015). Using SRH as an outcome covers a more holistic picture of health than any one particular disease measure, so it serves as a good starting point in synthesizing the aforementioned literatures.

The role of BMI is a bit more complex. In health literature, obesity has typically been studied as a health outcome (Austin et al. 2009, 2013; Barefoot, Warren, and Smalley 2015; Bauer and Jairam 2008; Boehmer and Bowen 2009; Cohen et al. 2017; Fredriksen-Goldsen, Kim, et al. 2013). However, obesity is also a risk factor for other morbidities and mortality (Daniels et al. 2005; Fredriksen-Goldsen, Emlet, et al. 2013; Solomon and Manson 1997). Hatzenbuehler, Phelan, and Link (2013) consider obesity a stigmatized characteristic that might also fundamentally contribute to population health disparities. In this dissertation, I explore obesity as a health outcome, setting myself up for future research considering its role on the causal pathway to self-rated health.

Similarly, access to a USOC has also been considered a health outcome in studies (Blosnich et al. 2014; Platt, Wolf, and Scheitle 2018), but in the fundamental cause framework, it can be considered a mediator to population level disease disparities since it is a resource hypothesized to affect health outcomes while access to it is determined by fundamental causes. I explore access to a USOC when one is sick or needs advice about health as a health outcome in this dissertation, and then suggest considering its role on the causal pathway to self-rated health in future research.

\section{RESEARCH QUESTIONS}

These are my research questions:

1. Overall: How does health vary by sexual orientation in the United States?

2. To what extent do self-rated health, body mass index, and access to a usual source of health care vary by the three components of sexual orientation - attraction, behavior, and identity - in the United States?

3. To what extent do self-rated health, body mass index, and access to a usual source of health care vary by the three components of sexual orientation - attraction, behavior, and identity - in the United States for males and females separately?

4. To what extent do self-rated health, body mass index, and access to a usual source of health care vary by age as well as the three components of sexual orientation-attraction, 
behavior, and identity - in the United States?

\section{RESEARCH DESIGN AND METHODS}

All three articles of this dissertation use quantitative research methods on nationally representative secondary data. Methods include descriptive statistics as well as logistic and multinomial regressions.

DATA

In this section I describe the dataset I use for my dissertation. I note the purpose of the survey, the survey design, the survey conductors, which files I use, specific key questions and responses, and the strengths and weaknesses of the data source. To avoid redundancy, each chapter's Methods section refers back to this section, but I discuss analyses in greater detail within each chapter.

Dataset: National Survey of Family Growth (NSFG; 2011-2019)

The National Survey of Family Growth (NSFG) is a repeated cross-sectional survey that collects data on the "family life, marriage and divorce, pregnancy, infertility, use of contraception, and general and reproductive health" of noninstitutionalized civilians (National Center for Health Statistics 2019). It is a "multi-stage probability based, nationally representative sample of the household population aged 15-44" (National Center for Health Statistics 2016) and expanded to include ages 15-49 in 2015 (National Center for Health Statistics 2019). Conducted by the Centers for Disease Control and Prevention's (CDC) National Center for Health Statistics (NCHS), the survey began in 1973 and was repeated periodically until 2006 when it changed to continuous interviewing over the course of the years in each corresponding survey (National Center for Health Statistics 2019). The in-person survey is conducted via computer assisted personal interviews (CAPI), but more sensitive questions, such as those about sexual orientation or sexual experiences, are completed via audio computer-assisted self-interviews (ACASI) (National Center for Health Statistics 2019; National Center for Health Statistics (NCHS) 2014, 2016, 2018, 2020). I use the 2011-2013, 2013-2015, 2015-2017, and 2017-2019 NSFG public use data files for males and females along with the corresponding files of sampling weights and survey design variables. ${ }^{2}$ I set up all files and conduct all analyses using Stata 14.2. ${ }^{3}$

This data is appropriate for this study because it has information on respondents' sexual

\footnotetext{
${ }^{2}$ Available for download here: ftp://ftp.cdc.gov/pub/Health_Statistics/NCHS/Datasets/NSFG

${ }^{3}$ Set up files available for download here: ftp://ftp.cdc.gov/pub/Health_Statistics/NCHS/Datasets/NSFG/stata/
} 
orientation identity, sexual attraction, and sexual behavior, as well as on the health outcomes of interest: self-rated health (SRH), body mass index (BMI), and access to a usual source of health care (USOC). All quotations and variables explained below were found in each Webdoc interactive codebook for their corresponding surveys; however, NCHS noted that as of December 31, 2020 all interactive Webdoc codebooks would be removed from the internet, so I made PDF copies of every page for variables that I use. ${ }^{4}$ Codebooks, documentation, and detailed information about the NSFG is available via their home page (https://www.cdc.gov/nchs/nsfg/index.htm).

\section{Measures}

Regarding sexual orientation identity, surveys 2011-2013, 2013-2015, and half of a random sample of 2015-2017 and 2017-2019 ask respondents: "Do you think of yourself as ..." (a) Heterosexual or straight, (b) Homosexual, gay, or lesbian, ${ }^{5}$ (c) Bisexual, (d) Not ascertained, (e) Refused, and (f) Don't know. This variable is called ORIENT for 2011-2015 and ORIENTA for 2017-2019. The other half of the random samples from 2015-2017 and 2017-2019 were asked: "Which of the following best represents how you think of yourself?" (a) Lesbian or gay; ${ }^{6}$ (b) Straight, that is, not lesbian or gay, ${ }^{7}$ (c) Bisexual, (d) Something else, (e) Not ascertained, (f) Refused, and (g) Don't know. This variable is called ORIENTB. To handle these question differences, I tested the distribution of sexual orientation identities between the two samples and, finding few differences, I recoded and combined the two samples. ${ }^{8}$

Regarding sexual attraction, each of the four surveys asks: "People are different in their sexual attraction to other people. Which best describes your feelings? Are you..." (a) Only attracted to males, ${ }^{9}$ (b) Mostly attracted to males, (c) Equally attracted to males and females, (d) Mostly attracted to females, (e) Only attracted to females, (f) Not sure, (g) Not ascertained, (h) Refused, and (i) Don't know. This variable is called ATTRACT.

\footnotetext{
${ }^{4}$ Access to Webdoc, the NSFG's interactive codebooks, was available on the following webpages, but do to their expected removal, I made PDF copies of each variable's page that are available upon request; all other NSFG codebooks and documentation are still available at these links: https://www.cdc.gov/nchs/nsfg/nsfg_2011 2013 puf.htm; https://www.cdc.gov/nchs/nsfg/nsfg 2013 2015 puf.htm; https://www.cdc.gov/nchs/nsfg/nsfg_2015_2017_puf.htm; https://www.cdc.gov/nchs/nsfg/nsfg_2017_2019 puf.htm

${ }^{5}$ For females; for males this response was "Homosexual or gay."

${ }^{6}$ For females; for males this response was "Gay."

${ }^{7}$ For females; for males this response was "Straight, that is, not gay."

${ }^{8}$ Analyses and results available upon request.

${ }^{9}$ For females; for males responses (a)-(e) were reversed.
} 
Regarding sexual behavior, each of the four female surveys has a computed variable of: "Whether R [respondent] has had any sexual experience with a female ${ }^{10}$ partner" (a) Yes, (b) No, (c) Not ascertained. It is composed from the answers to three other questions about same-sex sexual activity. This question was only applicable for female respondents who "gave answers other than 'don't know' or 'refused' to all of the questions on same-sex activity." Each of the four male surveys has a computed variable of: "Ever had oral or anal sex with a male" (a) Yes, (b) No, and (c) Not ascertained. It is composed from the answers to four other questions regarding same-sex activity for 2011-2013, 2013-2015, and 2015-2017; for 2017-2019 it is composed of five other questions regarding same-sex activity (the fifth question asks "Have you ever had any other sexual experience of any kind with another male?"). This question was only applicable for male respondents who "gave answers other than 'don't know' or 'refused' to all of the questions on same-sex activity." 11 This variable is called SAMESEXANY. For consistency across samples, I created a variable using the male 2017-2019 survey that only considered the same four questions considered by the previous surveys which I then used in subsequent analyses.

Regarding body mass index (BMI), the 2011-2013, 2013-2015, and 2015-2017 surveys provides a calculated body mass index (BMI) for respondents who had reported their height and weight. This variable is called BMI. However, this variable only reports whole number BMI values. According to the Centers for Disease Control and Prevention (2020) BMI of less than 18.5 is considered underweight, 18.5 to 24.9 is considered normal weight, 25.0-29.9 is considered overweight, and 30+ is considered obese. The variable BMI was constructed from the variables RWEIGHT and INCHES, so I created a new BMI variable that included one decimal place by following the CDC BMI formula (Centers for Disease Control and Prevention 2019). As such, I was able to combine the previous survey responses with the 2017-2019 data. The 2017-2019 public use survey reports BMI in categorical form as (a) Underweight, (b) Normal Weight, (c) Overweight, (d) Obese, and (e) Undefined BMI. For each of the four surveys, there are also missing values of "inapplicable." This variable is called BMICAT (U.S. Department of Health and Human Services 2020). For females, this measure is only applicable for those "older than 19 years and not currently pregnant." For males, this measure is only applicable for those 20

\footnotetext{
${ }^{10}$ For females; for males this computation is "Ever had oral or anal sex with a male."

11 Ibid.
} 
years or older.

Regarding access to a usual source of health care (USOC), each of the four surveys asks: "Is there a place you usually go to when you are sick or need advice about health?" (a) Yes, (b) No, (c) Refused, and (d) Don't know. This variable is called USUALCAR.

Regarding self-rated health (SRH), each of the four surveys asks: "In general, how is your health? Would you say it is ...” (a) Excellent, (b) Very good, (c) Good, (d) Fair, (e) Poor, (f) Not ascertained, (g) Refused, and (h) Don't know. This variable is called GENHEALT.

Inspired by the study by Boehmer and colleagues (2007), I also use controls for survey cycle, age, race/ethnicity, residence, foreign nativity, education, insurance, poverty, and sex. ${ }^{12}$ Parity was not included due to the inclusion of male respondents in the sample. I attempted to include smoking behavior in the analyses, but the male surveys for 2011-2013 and 2013-2015 neglected to ask questions regarding this important health behavior. Survey cycle is a categorical variable depicting the cycle (e.g., 2011-2013) in which the respondents participated. Age is a categorical variable with two levels (age 25-34 and age 35-44) to compare across chapters; Chapter 3 splits the sample into these two age groups to investigate age's effect on the healthrelated outcomes. Race/ethnicity is a four-level categorical variable: 1) Non-Hispanic White, 2) Hispanic, 3) Non-Hispanic Black, and 4) Non-Hispanic Other. Residence is a three-level categorical variable for those living in a principal city of a metropolitan statistical area (MSA), those in another area of an MSA, and those not in an MSA. Nativity is a dichotomous variable of US-born or foreign-born respondents. Education has four levels: 1) some high school or less, 2) high school diploma or GED, 3) some college, and 4) college or graduate degree. Insurance has four levels: 1) private or Medi-Gap, 2) Medicaid, CHIP, or state-sponsored insurance, 3) Medicare, military, or government insurance, and 4) single-service, Indian Health Service, or not covered by insurance. Poverty is measured by household income percent of the federal poverty level as $0-99 \%, 100-299 \%$, or $>300 \%$. Finally, sex is whether or not the NSFG included them in the male or female respondent data.

These datasets have several strengths to note. They allow me to examine each of the three elements of sexual orientation as opposed to just one. It is nationally representative with a large sample of sexual orientation minority respondents that furthers allows for separation of identities

\footnotetext{
${ }^{12}$ Boehmer and colleagues (2007) use these control variables, except for sex, in their study investigating overweight and obesity outcomes by sexual orientation identity among non-pregnant females.
} 
(e.g., instead of examining all sexual minorities grouped together versus heterosexuals, I can explore differences within the sexual minority group) for analyses. The surveys are recent (20112019) so information is more relevant. However, there are some weaknesses of these datasets as well. Respondents from the years before and after same-sex marriage legalization in 2015 are combined into one sample. Respondents are already separated by male and female with no nonbinary sex or gender reporting options. Population health scientists have also demonstrated the importance of context in determining health behaviors and outcomes, but these surveys do not have publicly available information on respondents' social or built environments. Despite these limitations, the following analyses still provide important country-level information about sexual minority health.

\section{CHAPTER 1 - HEALTH DISPARITIES BY SEXUAL ATTRACTION, BEHAVIOR, AND IDENTITY IN THE UNITED STATES \\ INTRODUCTION}

Ample research has found connections between fundamental social conditions - such as race, gender, and socioeconomic status - and health behaviors or health outcomes. These relationships remain consistent over time despite advances in information, medicine, and technology because minority groups (e.g., women) within each social condition (e.g., gender) often have less access to these and other health-promoting resources while enduring more stressors compared to majority group members. A growing body of quantitative research has also been showing a connection between the social condition of sexual orientation and health-related factors. Minority stress theory typically drives these studies by postulating that sexual minorities experience additional stressors due to stigma, prejudice, and discrimination that create stressful contexts leading to mental health issues. Both lines of theorizing work together to explain the higher rates of poor health often reported by sexual minorities compared to their heterosexual counterparts.

Sexual minority health research has often focused on connections to mental health and/or substance use/abuse and has been hampered by methodological issues such as convenience samples, small sample sizes, and surveys ignoring questions about sexual orientation altogether. To help remedy the need for nationally representative information on sexual minority health, I use the National Survey of Family Growth (NSFG; 2011-2019) which asks about three components of sexual orientation along with a variety of demographic, socioeconomic, and 
health-related questions over the course of four cross-sectional survey waves. I explore how sexual orientation identity, sexual attraction, and sexual behavior are associated with self-rated health (SRH), body mass index (BMI), and access to a usual source of health care (USOC). The findings in this dissertation chapter add to the sexual minority health literature by considering fundamental cause theory and minority stress theory as both would suggest significant differences in the health-related outcomes between stigmatized and non-stigmatized groups.

\section{LITERATURE REVIEW}

Link and Phelan's (1995) fundamental cause theory examines the pervasive connections between fundamental social conditions and health disparities. While noting it has been helpful to uncover individual risk factors and behaviors such as poor diet, lack of exercise, and smoking that lead to poor health, fundamental cause theory refocuses the conversation to consider "what puts people at risk of risks" that lead to poor health (Link and Phelan 1995:80). In other words, they focus on the importance of environments or contextual elements in helping or hindering people from engaging in healthful behaviors. They briefly discuss the potential for social statuses such as race and gender to be fundamental social conditions since research shows racial and gendered gaps in health outcomes, but their paper spotlights socioeconomic status since they can plainly illustrate how this social factor affects access to various resources.

A key characteristic of a fundamental cause of health disparities is that it affects access to resources that can limit exposure to risk factors of disease (e.g., money to live in a clean house, knowledge to avoid using tobacco) and provide better means of coping with disease if contracted (e.g., social networks with doctors to offer more personalized help). Briefly mentioned in their original paper and elaborated on in a later paper, stigmatization can also be considered a fundamental cause (Hatzenbuehler et al. 2013; Link and Phelan 1995). Stigmatization affects access to health-related resources and it does so consistently "across time and place" by influencing "multiple disease outcomes through multiple risk factors among a substantial number of people" (Hatzenbuehler et al. 2013:813). Therefore, and as suggested by Hatzenbuehler et al. (2013), other stigmatized statuses such as sexual orientation can be considered a fundamental cause of disease if they also meet these criteria.

A growing body of quantitative research demonstrates a relationship between sexual orientation and health behaviors and outcomes. However, sexual minority health research has often focused on connections to mental health (e.g., Barnes et al. 2014; Mays and Cochran 2001; 
Meyer 2003; Plöderl and Tremblay 2015) and/or substance use/abuse (e.g., Bauer, Jairam, and Baidoobonso 2010; Brewster and Tillman 2011; Chaudhry and Reisner 2019; Paschen-Wolff et al. 2019) using a minority stress model perspective. Meyer (2003) proposed minority stress theory as a framework for understanding the higher levels of mental distress often found within sexual minority groups compared to their heterosexual counterparts. This theory elaborates on the excess stress stigmatized minority groups endure that lead to mental distress and disorders by referring to minority stress as unique (i.e., applies only to stigmatized people in addition to general stressors), chronic (i.e., "related to relatively stable underlying social and cultural structures"), and socially based (i.e., "stems from social processes, institutions, and structures beyond the individual") (Meyer 2003:676). While the original article on this conceptual framework briefly mentions somatic illness as a consequence of stress as well, the overall focus has been on mental health outcomes.

A review article published a decade later explored burgeoning evidence of poorer physical and general health among sexual minorities compared to their heterosexual counterparts (Lick et al. 2013). Lick et al. (2013) proposed using a minority stress framework to explain such findings. More recent work found partial support for "the hypothesized negative effects of minority stress on the physical health of sexual minorities [emphasis added]," but notes the need for more research in this area (Frost, Lehavot, and Meyer 2015:7). Flenar, Tucker, and Williams (2017) examined a sample of sexual minorities guided by the minority stress model finding sexual minority stress was negatively related to their engagement in a health-promoting lifestyle and positively related to their number of physical health problems. However, these analyses were not conducted on a nationally representative study and did not include a heterosexual comparison group like this study does.

Both fundamental cause theory and minority stress theory discuss the importance of resources when it comes to managing health, the importance of understanding context as it applies to risk exposure, and stigma as it relates to each. Fundamental cause theory specifically states the "money, knowledge, power, prestige, and the kinds of interpersonal resources embodied in the concepts of social support and social network" are what make certain social causes of disease so fundamental (Link and Phelan 1995:87). Different socially based structures are what allow for group disparities in access to these resources. "The situation of being stigmatized depletes many of these same resources" (Hatzenbuehler et al. 2013:814) while 
minority stress can "overwhelm coping resources and compromise well-being" (Frost et al. 2015:528). Meyer's (2003) model starts with situating minority stress in the general environment and highlights the importance of considering other factors (e.g., socioeconomic status, minority status) that would affect "exposure to stress and coping resources" (678); fundamental cause theory calls for researchers to “contextualize risk factors by asking what it is about people's life circumstances that shapes their exposure to such risk factors" (Link and Phelan 1995:85). In their suggestions for future research, Hatzenbuehler et al. (2013) name datasets that have information on stigma and social environment context noting the need to evaluate their interactions. Although this dissertation cannot comprehensively explore location-specific contextual elements, it considers socioeconomic status and various components of sexual orientation as they relate to multiple health-related factors and outcomes.

Health disparities exist between sexual orientation minorities and their heterosexual counterparts (Dilley et al. 2010). However, many studies examining these relationships have been inconsistent and incomplete in their operationalizations of sexual minorities and hampered by convenience samples, small sample sizes, and surveys ignoring questions about sexual orientation altogether (Gates 2011; Gonzales, Przedworski, and Henning-Smith 2016; Hottes et al. 2016; Meyer 2003). Nascent analyses included multiple sexual minority identities together in one sexual minority category (usually gay, lesbian, and bisexual), though recent work has shown there are differences in health-related behaviors and outcomes between these groups. Research also suggests that sexual orientation is composed of three components - attraction, behavior, and identity - and that simply measuring the sexual minority community varies by each. For example, estimates of same-sex attraction and behavior are generally higher than estimates of a sexual minority identity (Brewster and Tillman 2011; Gates 2011). Each component should be considered when exploring their associations with health factors. Here I provide an overview of how each of these three components are related to three health-related outcomes - self-rated health, BMI, and access to a usual source of health care.

Early research into sexual minority health often grouped non-heterosexual identities together, often due to data sample size limitations. For example, Garofalo and colleagues (1998) took advantage of the 1995 Massachusetts Youth Risk Behavior Survey (YRBS) conducted by the Centers for Disease Control and Prevention (CDC) which was the first time a question about sexual orientation was used in this standardized survey. Only $2.2 \%(n=104)$ of the sample 
responded as lesbian, gay, or bisexual (LGB) so they were grouped together and race and gender interactions were not included in the $\chi^{2}$ analyses. Even early work with large nationally representative samples were hampered by the need to compare one LGB group to heterosexuals as seen in Mays and Cochran's (2001) study which used the National Survey of Midlife Development in the United States (MIDUS). While useful in demonstrating health differences due to stigmatization of sexual orientation as a whole, the sexual minority group is not homogeneous (Institute of Medicine 2011).

Differences between sexual minority groups are important. For instance, bisexual adults are also at risk of biphobia which is "negative attitudes about bisexuality and bisexual individuals" (Bennett 1992; as cited by Mulick and Wright, Jr. 2002:47) from both heterosexuals and from lesbians and gay men within the sexual minority community (Mulick and Wright, Jr. 2002). This type of "double discrimination" (Ochs 1996; as cited by Mulick and Wright, Jr. 2002:47) means that while lesbians and gay men may be able to find support and coping resources within the sexual minority community, bisexuals may not. This additional stigma and discrimination can further influence negative health outcomes.

To compensate for nationally representative surveys that did not ask respondents about sexual orientation identity, scholars have looked at sexual behavior (e.g., Faulkner and Cranston 1998) or cohabitation for guidance (e.g., Smith and Gates 2001). Health disparities are also apparent by sexual behavior differences displaying negative health outcomes for those participating in same-sex behavior (Cochran and Mays 2014; DuRant, Krowchuk, and Sinal 1998; Faulkner and Cranston 1998). The few studies that have differentiated between sexual orientation attraction, behavior, and identities have found disparities between component prevalence within a sample and subsequent health outcomes (Wolff et al. 2017). For example, Brewster and Tillman (2011) found more respondents reported same-sex attraction and behavior than identities as lesbian, gay, or bisexual. Their analyses also found differences in the association between each component and substance use (Brewster and Tillman 2011). PaschenWolff and colleagues (2019) explored trends in health disparities such as substance use and sexual risk behaviors among women and found associations between each sexual orientation component and the health outcomes were different and changed over time. To my knowledge, no study has considered all three components and the specific health outcomes of SRH, BMI, and access to a usual source of health care. This chapter addresses that gap in the literature. 


\section{RESEARCH QUESTION}

1. To what extent do self-rated health, body mass index, and access to a usual source of health care vary by the three components of sexual orientation - attraction, behavior, and identity — in the United States?

\section{METHODS}

Data

Detailed descriptions of the datasets and measures used from the National Survey of Family Growth (NSFG; 2011-2019) are available in the Data section of this document. I used the same data throughout this dissertation, but the analyses differ in each chapter.

Analyses

I produced descriptive statistics by each of the three sexual orientation components. The proportions were weighted to create estimates for the US population of noninstitutionalized men and non-pregnant women aged 25-44 years. I include the $p$-values determined by the "Pearson $\chi^{2}$ test with the Rao and Scott $(1981,1984)$ second-order correction" or the $F$ test (the default output from the Stata svy tabulate command) in these tables that indicate independence between groups (stata.com n.d.:11). I also produced an unweighted descriptive table for the adult sample broken up by sexual orientation component and each of the three health-related outcomes. It includes $p$-values determined by Pearson $\chi^{2}$ tests (and Fisher's exact two-tailed tests for $2 \times 2$ tables) comparing subgroups within each status. Percentages are provided for each category for SRH (excellent; very good; good; fair; poor), BMI (underweight, $<18.5$; normal weight, 18.5-24.9; overweight, 25.0-29.9; obese, >30.0), and USOC (yes; no), but the statistical tests use the dichotomous SRH (excellent, very good, and good; fair and poor) and multinomial BMI (underweight and normal weight, $<24.9$; overweight, 25.0-29.9; obese, >30.0) outcome categorizations also used in the regressions due to small cell sizes and examples from pervious literature (e.g., Boehmer et al. 2007; Strutz et al. 2015).

I performed nine logistic regressions that regressed SRH (binomial), BMI (multinomial), and access to a USOC (binomial) on sexual orientation identity, sexual attraction, and sexual behavior separately. Dichotomizing SRH has produced similar results to other methods of 
analyses (Manor, Matthews, and Power 2000) ${ }^{13}$ and has been used in recent similar work (Solazzo, Gorman, and Denney 2020; Strutz et al. 2015). The article which inspired this dissertation analyzed BMI via multinomial logistic regression by combining those who are underweight $(<18.5)$ and normal weight (18.5-24.9) into one category, those who are overweight (25.0-29.9) into another category, and those who are obese (30.0+) into a third category-I took this same approach in my analyses (Boehmer et al. 2007). The question asking respondents whether they had access to a USOC or not only had two non-missing responses (yes and no), so I analyzed it via logistic regression. I controlled for demographic, socioeconomic, and healthrelated variables.

To prepare the data for analyses, I appended the female respondent data from 2011-2013, 2013-2015, 2015-2017, and 2017-2019 together as well as the male data over the same years to produce two datasets for the nine-year period. I then appended the two sets of data together so it contained all four survey waves for females and all four for males. Since the NSFG uses a complex sampling design, I also downloaded the corresponding weight files containing the appropriate sample weights and survey design variables. I used the Stata svyset command with the appropriate weight variable and the corresponding strata and primary sampling unit (psu) variables. Detailed instructions on combining NSFG data can be found here. ${ }^{14}$

To compare the same samples across tests, I dropped those missing any sexual orientation measures or those who responded "refused," "don't know," "not sure," or "something else" as is common in the literature (Barnes et al. 2014; Blosnich and Bossarte 2012; Chaudhry and Reisner 2019; Scheitle and Wolf 2018). Since I used BMI as a control variable, I dropped females who were pregnant because "BMI is not considered to be appropriate to measure obesity and overweight" for them (Boehmer et al. 2007). I also dropped those under age 25 to appropriately consider the control variable on highest education completed. Weights were not assigned across all four surveys to those who were older than age 45, so anyone over 45 was also dropped. All analyses were conducted in Stata 14.2 and all descriptive and regression tables can be found in the Appendix.

\footnotetext{
${ }^{13}$ However, the response they dichotomized was from a SRH variable with four potential values (combined poor and fair, and combined excellent and good).

${ }^{14} \mathrm{https} / / /$ www-cdc-gov.www.libproxy.wvu.edu/nchs/nsfg/nsfg_combining_data.htm
} 


\section{RESULTS}

Weighted descriptive statistics are presented in Tables 1-A to 1-C in the Appendix. According to Table 1-A, the sexual orientation identity groups significantly differ from one another in their sexual attraction, sexual behavior, sex, age, residence, nativity, insurance, household income, SRH, BMI, and survey cycle year. There are no significant differences in sexual orientation identity groups by race/ethnicity, education, or USOC. According to Table 1$\mathrm{B}$, the sexual attraction groups significantly differ from one another in every demographic, socioeconomic, and health-related measure. The same is true for those who have or have not had same-sex sexual experiences according to Table 1-C.

There are more males than females who report either a heterosexual $(51.8 \%$. $\mathrm{CI}=50.8$, $52.8)$ or gay/lesbian $(53.2 \%, \mathrm{CI}=46.6,59.6)$ identity, but the vast majority of bisexuals report being female $(82.8 \%, \mathrm{CI}=78.8,81.2)$. Age is fairly evenly split between $25-34$ and 35-44 years old for heterosexuals, but the majority of gay/lesbian $(58.6 \%, \mathrm{CI}=52.2,64.8)$ and bisexual $(65.3 \%, \mathrm{CI}=61.3,69.2)$ are in the younger age group. Those reporting attraction to mostly the opposite sex or equally to males and females were much more likely to be female $(78.1 \%$, $\mathrm{CI}=75.5,80.4 ; 83.0 \%, \mathrm{CI}=77.0,87.7$, respectively) while those reporting attraction to only the same or opposite sex were slightly more likely to be male $(54.4 \%, \mathrm{CI}=46.9,61.8 ; 54.3, \mathrm{CI}=53.3$, 55.4 , respectively). Those reporting attraction to mostly the opposite sex $(62.4 \%, \mathrm{CI}=59.4,65.4)$, equal attraction $(67.9 \%, \mathrm{CI}=62.8,72.6)$, and only attracted to the same sex $(60.7 \%, \mathrm{CI}=54.0$, 66.9) were more likely to be younger. Females were more likely to report having a same-sex experience $(74.6 \%, \mathrm{CI}=72.2,76.9)$ than not $(45.7 \%, \mathrm{CI}=44.7,46.7)$ while males were more likely not to engage in same-sex behavior $(54.3 \%, \mathrm{CI}=53.3,55.3)$ than to engage $(25.4 \%$, $\mathrm{CI}=23.1,27.8)$. Younger participants were more likely to report having a same-sex experience $(56.9 \%, \mathrm{CI}=54.3,59.4)$ than not $(50.7 \%, \mathrm{CI}=49.5,51.8)$ while older respondents were more likely not to engage in same-sex behavior $(49.3 \%, \mathrm{CI}=48.2,50.5)$ than to engage $(43.1 \%$, $\mathrm{CI}=40.6,45.7)$.

The unweighted descriptive table (Table 1-D) shows significant variation in each healthrelated outcome by sexual orientation component, sex, and age. Using the dichotomized version of SRH, heterosexual or straight (hereafter: heterosexual) respondents significantly differ from both homosexual, gay, or lesbian (hereafter: gay/lesbian) and bisexual respondents. However, those who are equally attracted to males and females differ from those who are only or mostly 
attracted to the opposite sex or only attracted to the same sex. Using the combined underweight and normal categories compared to the separate overweight and obese categories, bisexual respondents differ from both heterosexual and gay/lesbian respondents; a similar pattern is seen in the sexual attraction categories. Access to a USOC only differs between heterosexual and gay/lesbian respondents. Those only attracted to the opposite sex differ from both "mostly" categories and those equally attracted to males and females, both "mostly" categories differ from one another, and those only attracted to the same sex differ from those mostly attracted to the same sex. There are significant differences in SRH, BMI, and USOC between those who have and have not had same-sex sexual experiences and between the sex and age groups.

In the full models (Tables 1-E to 1-G), results show that compared to their heterosexual counterparts (a) bisexuals have significantly lower odds of reporting good, very good, or excellent (vs. poor or fair) $\mathrm{SRH}(\mathrm{OR}=0.65, \mathrm{CI}=0.51,0.83)$; (b) those equally attracted to males and females have significantly lower odds of reporting good, very good, or excellent SRH $(\mathrm{OR}=0.62, \mathrm{CI}=0.47,0.82)$; (c) those who have had sexual experience with a same-sex partner have significantly lower odds of reporting good, very good, or excellent SRH $(\mathrm{OR}=0.70$, $\mathrm{CI}=0.58,0.85$ ). Results show that compared to their heterosexual counterparts (a) bisexuals are significantly at greater relative risk of being obese $(\mathrm{RRR}=1.28, \mathrm{CI}=1.02,1.62)$; (b) there are no significant differences in relative risk of being overweight or obese by sexual attraction or sexual behavior. There are no significant differences in odds of having access to a USOC by any of the sexual orientation components.

Table 1. Summary Table of Significant Findings $(\mathrm{N}=\mathbf{2 3 , 5 6 7})$

\begin{tabular}{|c|c|c|c|c|c|c|c|c|c|c|}
\hline \multirow{3}{*}{ Health Factor } & \multicolumn{10}{|c|}{ Sexual Orientation Component } \\
\hline & \multicolumn{5}{|c|}{ Attraction } & \multicolumn{2}{|c|}{ Behavior } & \multicolumn{3}{|c|}{ Identity } \\
\hline & $\mathbf{O}$ & MO & $\mathbf{E}$ & MS & $\mathbf{S}$ & $\mathbf{N}$ & $\mathbf{Y}$ & $\mathbf{H}$ & $\mathbf{L} / \mathbf{G}$ & $\mathbf{B}$ \\
\hline SRH (excellent-good) & & & - & & & & - & & & - \\
\hline BMI (overweight) & & & & & & & & & & \\
\hline BMI (obese) & & & & & & & & & & + \\
\hline USOC (yes) & & & & & & & & & & \\
\hline
\end{tabular}

Note: $+=$ significant positive association; - = significant negative association; $\mathrm{O}=$ only attracted to the opposite sex; $\mathrm{MO}=$ mostly attracted to the opposite sex; $\mathrm{E}=$ equally attracted to males and females; $\mathrm{MS}=$ mostly attracted to the same sex; $\mathrm{S}=$ only attracted to the same sex; $\mathrm{N}=$ no same-sex behavior; $\mathrm{Y}=\mathrm{yes}$, same-sex behavior; $\mathrm{H}=$ heterosexual or straight; $\mathrm{L} / \mathrm{G}=$ lesbian or gay; $\mathrm{B}=$ bisexual; $\mathrm{SRH}=$ self-rated health (excellent, very good, or good); $\mathrm{BMI}=$ body mass index; $\mathrm{USOC}=$ access to usual source of care. 


\section{DISCUSSION}

While the lack of significant differences in access to a USOC and most BMI outcomes is encouraging, the disparities in SRH are disconcerting due to the SRH's strong connection with morbidities and mortality (Ferraro et al. 1997; Jylhä 2009). Somewhat unexpectedly, my results show that gay and lesbian respondents did not have a significant difference in SRH from their heterosexual counterparts. However, these findings are not unheard of in this literature. Gorman and colleagues (2015) used probability data from the Behavioral Risk Factor Surveillance survey (BRFSS) across seven U.S. states, and perhaps even more counterintuitively found that gay men and lesbians reported lower rates of poor SRH than heterosexuals. Also, similar to my results, Gorman and colleagues (2015) found bisexual men and women reporting significantly poorer SRH compared to their heterosexual counterparts. They state that these outcomes were mainly accounted for by poorer socioeconomic conditions among bisexuals (Gorman et al. 2015) which can also be seen in my descriptive tables where bisexuals are at a disadvantage in household income and insurance, though not necessarily in educational attainment.

That only one significant difference in BMI was found is not too unpredictable given the mixed findings in the literature, particularly by gender (Blosnich et al. 2014). For example, Conron, Mimiaga, and Landers (2010) found gay/lesbian respondents less likely to be overweight compared to heterosexuals, but when broken down by sex, lesbian women were no longer significantly different from their heterosexual counterparts while gay men were still less likely to be overweight. They also found gay men were less likely to be obese while lesbian women were more likely to be obese compared to their heterosexual counterparts which negated any significant differences considering them together, and that bisexuals did not differ from heterosexuals for either weight class when considered together or separately by sex (Conron et al. 2010). Another study found no differences between sexual minority orientation identities of being overweight/obese (i.e., $\mathrm{BMI} \geq 25$ ), but when looking at obesity specifically (i.e., BMI $\geq 30$ ), multiple differences emerged by subgroups differentiated by gender and identity (Warren, Smalley, and Barefoot 2016). Newlin Lew, Dorsen, and Long (2018) found gay men were less likely to be obese than straight men, but found no differences between bisexual men and straight men. Boehmer et al. (2007) used an earlier version of the NSFG and found that compared to heterosexual women, only lesbians had higher odds of being overweight and obese while bisexual women did not. That these analyses found no differences between gay/lesbian 
respondents and heterosexuals in weight status might be reflected in the mixed findings of higher weight or no weight difference between sexual minority women and their sexual majority counterparts (e.g., Bowen et al. 2008) and lower weight in sexual minority men (e.g., Blosnich et al. 2014); the significant finding of higher relative risk of obesity in bisexuals is interesting due to the lack of findings in the literature (e.g., Blosnich et al. 2014) and perhaps is a reflection of biphobia. It is probable that a more detailed exploration of these NSFG surveys by sex would reveal significant differences in BMI by sexual orientation components.

It was somewhat unexpected to find that no element of sexual orientation was associated with the odds of having a USOC. Previous research has found some disparities (Blosnich et al. 2014, 2014; Conron et al. 2010; Dilley et al. 2010), though often the findings are mixed by gender or sexual orientation identity and do not utilize nationally representative data. For example, Blosnich and colleagues (2014) found differences in gender by their likelihood of having a routine check-up compared to their heterosexual counterparts. Conron and colleagues (2010) found differences in sexual orientation regarding their odds of not having a regular provider. Dilley and colleagues (2010) found differences by gender and sexual orientation exploring the likelihood of having a personal doctor or receiving more specialized care. Additionally, the NSFG question about a USOC did not ask about the quality of health care available and the question itself is vague enough that respondents might consider the internet to be a "place" they can go to for "advice about health." However, it should be noted that every other control variable explored had differences within respondents' values with regards to access to a USOC. Further exploration within the NSFG datasets by sex could shine light on why there were no significant differences in this analyses.

The analyses in this chapter have several strengths for the sexual minority health literature. To my knowledge, this is the first study to use a nationally representative samples of males and females that analyzes all three elements of sexual orientation: attraction, behavior, and identity and their association with self-rated health, BMI, and access to a usual source of health care. Whereas similar studies focus on substance use/abuse or health risk behaviors (Bauer et al. 2010; Boehmer et al. 2007; Brewster and Tillman 2011; Paschen-Wolff et al. 2019); my study focuses on a more holistic measure of health via self-rated health, a physical health outcome via BMI, and a health resource-related outcome via access to a USOC. Exploring all three components of sexual orientation also adds to the sexual minority health literature by examining 
health disparities from multiple perspectives.

While informative, these analyses also have limitations that should be considered and addressed in future research. For example, the public datasets from NSFG have extremely limited contextual data; thus, I cannot examine how social or built environments are also affecting self-rated health. Extensive research shows it is important to consider the connections between cultural, historical, physical, political, and social contexts and health outcomes (Bernard et al. 2007; Cummins et al. 2007; Link and Phelan 1995), but these relationships cannot be explored with the current public use data. This analyses also examines males and females together, only controlling for sex. Research has shown there are differences between males and females by sexual orientation regarding health-related outcomes (Chaudhry and Reisner 2019; Plöderl and Tremblay 2015).

\section{CONCLUSION}

Health disparities exist by sexual orientation. Fundamental cause theory and minority stress theory are two frameworks for understanding why this is - stigma resulting in excessive minority stress. Using nationally representative data to explore the relationships between selfrated health (SRH), body mass index (BMI), and access to a usual source of health care (USOC) by sexual orientation components (i.e., attraction, behavior, and identity) reveals significantly poorer SRH across various sexual minority statuses, only one significant difference in regards to BMI (bisexuals have higher odds of being obese), and no disparities in access to a USOC by any component. However, the sexual minority health literature suggests that there may be further differences when considering minority groups by sex/gender. Chapter 2 disentangles the roles sex plays in the relationships between sexual orientation and SRH, BMI, and access to a USOC. 
CHAPTER 2 - HEALTH DISPARITIES BY SEXUAL ORIENTATION COMPONENTS AMONG A NATIONALLY REPRESENTATIVE SAMPLE OF MALES AND FEMALES IN THE UNITED STATES

INTRODUCTION

Fundamental social conditions — such as race, gender, and socioeconomic status - have consistently influenced health behaviors and outcomes because they affect access to a variety of resources that can improve or maintain health or can mitigate disease effects. Sexual minority health literature considers how sexual orientation affects access to health-related resources and has recently been involved in discussions naming stigma as a fundamental social condition. Minority stress theory highlights the excessive amount of stressors that sexual minorities endure due to their stigmatized group status which results in prejudice and discrimination against them. These extra stressors can then manifest in health problems. It is important to keep both theories in mind when examining sexual minority health.

Research on sexual minority health has found not only disparities between sexual orientations, but within them as well, particularly by sex and gender. Sexual minority men and women can face different types of prejudice and discrimination and the literature is quite sparse regarding those who do not identify within the sex and/or gender binary. Differences by sex can be hidden in analyses that consider sexual minorities as a homogenous group. This chapter digs deeper into the previous chapter's findings by exploring how each health outcome-self-rated health (SRH), body mass index (BMI), and access to a usual source of health care (USOC) vary for females and males by sexual orientation components - sexual attraction, sexual behavior, and sexual orientation identity. Using the National Survey of Family Growth (NSFG; 2011-2019) again, this chapter runs the same analyses, but keeps the male and female datasets separate. The results show only one same significant relationship, several same non-significant relationships, and a few differences in significant relationships between females and males which supports the need to consider sex and sexual orientation when studying sexual minority health.

\section{LITERATURE REVIEW}

It is important to consider the intersectionality of statuses or characteristics when exploring health disparities. Attitudes, and subsequent discrimination, toward sexual minorities can vary by the subgroup's sexual orientation and gender under consideration such as lesbians, gay men, bisexual men, and bisexual women (Worthen 2013). In addition to homophobia or 
heterosexism, sexual minority women have to contend with sexism and misogyny which can contribute to adverse health outcomes (Szymanski 2005). Sexual minority men and women may also differ in the way they internalize heterosexism due to differences in gender socialization (c.f., Mayfield 2001; Szymanski and Chung 2001; as cited by Szymanski, Kashubeck-West, and Meyer 2008). Internalized heterosexism - "or the internalization of negative messages about homosexuality by lesbian, gay, and bisexual (LGB) people"-is significantly correlated with fewer social supports and poorer mental and psychosocial health, and the few available studies suggest a link to physical health as well (Szymanski et al. 2008:525). Sexual minority health research has found that coping and other health-related behaviors and outcomes vary by sexual orientation and sex.

Previous studies exploring sexual minority health by sex have focused on mental health and substance use. Chaudhry and Reisner (2019) used a national probability study to explore major depressive episodes and substance abuse or dependence. Overall, they found that bisexual adults fared worse than their other sexual minority and heterosexual counterparts, females had a higher prevalence of major depressive episodes than males, and most males (except bisexual females, demonstrating their double burden) had higher prevalences of alcohol and illicit drug abuse or dependence compared to their female sexual orientation counterparts (Chaudhry and Reisner 2019). However, a review article on mental health among sexual minorities reported mixed results regarding increased alcohol use among sexual minority men, but did note "elevated risks for depression, anxiety, suicide attempts or suicides, and substance-related problems for SM [sexual minority] men and women" (Plöderl and Tremblay 2015:367). Fish (2019) explored differences in high-intensity binge drinking by sexual attraction, behavior, and identity. She found that for sexual minority women, they were more likely to engage in high-intensity binge drinking regardless of sexual orientation component while the outcome varied for sexual minority men based on sexual orientation component. A review paper on tobacco use among sexual minorities also reported mixed results regarding the prevalence of smoking among bisexual men, but found other sexual minority men and women are more likely to smoke than their heterosexual peers (Lee et al. 2009). Cochran and Mays (2014) considered differences in sexual behavior by men and women and found greater suicide mortality risk for women who have sex with women compared to presumptively heterosexual women, but found no difference between like groups for men. These studies highlight the continued need to consider differences 
between sexual minority groups and sex differences within them.

Blosnich and colleagues (2014) found that all sexual minority groups stratified by gender (i.e., lesbian women, bisexual women, gay men, bisexual men) were more likely to be current smokers than their heterosexual counterparts, but other health-related differences were found only by one group compared to their heterosexual peers depending on the outcome in question. For example, pertinent to this chapter, gay men were statistically significantly less likely to be overweight or obese compared to their heterosexual counterparts, but no significant differences in these weight categories were found between bisexual men, lesbians, or bisexual women and their heterosexual counterparts. However, their findings were based on surveys from ten US states. Boehmer and colleagues (2007) used a nationally representative survey (females only) and found that lesbians, but not bisexuals or those reporting "something else," were more likely to be overweight or obese than their heterosexual peers. A systematic review of research on sexual minority women and obesity by Bowen et al. (2008) found nine studies reporting lesbians were more often overweight or obese than their heterosexual counterparts, five studies finding no differences between them, and four studies with no comparison group; though, none of the studies examined were population-based.

Regarding the other health outcomes explored in this chapter, recent research has explored self-rated health (SRH) among sexual minorities by gender. Using the Behavioral Risk Factor Surveillance survey (BRFSS) across seven U.S. states, Gorman and colleagues (2015) found bisexual men and women reported the highest rates of poor SRH which were mainly accounted for by their poor socioeconomic conditions. Conversely, gay men and lesbians reported lower rates of poor SRH than heterosexuals (Gorman et al. 2015). Predicted probabilities of SRH differed significantly between gay men and bisexual men and between lesbian women and bisexual women, but they did not differ between gay men and lesbians nor between bisexual men and bisexual women (Gorman et al. 2015). However, an earlier study with a smaller sample found gay men, lesbians, and bisexual women each had higher odds of reporting poor physical and mental health than their heterosexual counterparts; bisexual men had higher odds of reporting poorer mental health than their heterosexual counterparts (Dilley et al. 2010). Using the 2013-2014 National Health Interview Surveys (NHIS), Gonzales et al. (2016) found lesbians had higher odds of reporting fair or poor SRH than their heterosexual counterparts, but no differences between gay men, bisexual men, or bisexual women and their 
heterosexual counterparts. This study adds to the mixed findings by using the nationally representative NSFG to further explore SRH among sexual minorities by sex.

The literature is also composed of mixed findings regarding having a usual source of health care. Previous research has found disparities dependent on gender and sexual orientation, though often not from nationally representative data (e.g., Blosnich et al. 2014, 2014; Conron et al. 2010; Dilley et al. 2010). For example, Blosnich and colleagues (2014) found lesbian and bisexual women less likely to have a routine check-up compared to their heterosexual counterparts, but no differences between sexual minority men and their heterosexual counterparts. Analyses by Conron and colleagues (2010) found bisexuals at greater odds of not having a regular provider while gay men and lesbians did not differ from their heterosexual counterparts. Dilley and colleagues (2010) found gay men as more likely to have a personal doctor than their heterosexual counterparts while lesbians and bisexuals did not differ; however, they did find some differences in having health insurance or receiving more specialized care.

Recent research shows that health disparities vary by sexual orientation components for males and females, but more work is needed to contribute to this growing area of interest. This chapter addresses both associations between the three components of sexual orientation and how these associations differ by sex for SRH, BMI, and access to a USOC.

\section{RESEARCH QUESTION}

1. To what extent do self-rated health, body mass index, and access to a usual source of health care vary by the three components of sexual orientation - attraction, behavior, and identity — in the United States for males and females separately?

\section{METHODS}

Data

Detailed descriptions of the datasets and measures used from the National Survey of Family Growth (NSFG; 2011-2019) are available in the Data section of this document. I used the same data throughout this dissertation, but the analyses differ in each chapter. Analyses

I produced descriptive statistics by each of the three sexual orientation components separately for females and males. The proportions were weighted to create estimates for the US population of noninstitutionalized men and non-pregnant women aged 25-44 years. I include the 
$p$-values determined by the "Pearson $\chi^{2}$ test with the Rao and Scott $(1981,1984)$ second-order correction" or the $F$ test (the default output from the Stata svy tabulate command) in these tables that indicate independence between groups (stata.com n.d.:11). I also produced an unweighted descriptive table for the adult sample broken up by sexual orientation component and each of the three health-related outcomes. It includes $p$-values determined by Pearson $\chi^{2}$ tests (and Fisher's exact two-tailed tests for $2 \times 2$ tables) comparing subgroups within each status. Percentages are provided for each category for SRH (excellent; very good; good; fair; poor), BMI (underweight, <18.5; normal weight, 18.5-24.9; overweight, 25.0-29.9; obese, >30.0), and USOC (yes; no), but the statistical tests use the dichotomous SRH (excellent, very good, and good; fair and poor) and multinomial BMI (underweight and normal weight, $<24.9$; overweight, 25.0-29.9; obese, >30.0) outcome categorizations also used in the regressions due to small cell sizes $^{15}$ and examples from pervious literature (e.g., Boehmer et al. 2007; Strutz et al. 2015).

I performed nine logistic regressions that regressed SRH (binomial), BMI (multinomial), and access to a USOC (binomial) on sexual orientation identity, sexual attraction, and sexual behavior separately for females and for males. Dichotomizing SRH has produced similar results to other methods of analyses (Manor et al. 2000) ${ }^{16}$ and has been used in recent similar work (Solazzo et al. 2020; Strutz et al. 2015). The article which inspired this dissertation analyzed BMI via multinomial logistic regression by combining those who are underweight $(<18.5)$ and normal weight (18.5-24.9) into one category, those who are overweight (25.0-29.9) into another category, and those who are obese $(30.0+)$ into a third category - I took this same approach in my analyses (Boehmer et al. 2007). The question asking respondents whether they had access to a USOC or not only had two non-missing responses (yes and no), so I analyzed it via logistic regression. I controlled for demographic, socioeconomic, and health-related variables.

To prepare the data for analyses, I appended the female respondent data from 2011-2013, 2013-2015, 2015-2017, and 2017-2019 together and appended the male data over the same years together to produce two datasets, one of female respondents and one of male respondents, for the nine-year period. Since the NSFG uses a complex sampling design, I also downloaded the

\footnotetext{
${ }^{15}$ Only three males equally attracted to males and females reported Medicare, military, or government insurance; only three males mostly attracted to the same sex reported less than a high school education. All other cell sizes for each sexual orientation component and variables used in analyses were $\geq 5$.

${ }^{16}$ However, the response they dichotomized was from a SRH variable with four potential values (combined poor and fair, and combined excellent and good).
} 
corresponding weight files containing the appropriate sample weights and survey design variables. I used the Stata svyset command with the appropriate weight variable and the corresponding strata and primary sampling unit (psu) variables. Detailed instructions on combining NSFG data can be found here. ${ }^{17}$

To compare the same samples across tests, I dropped those missing any sexual orientation measures or those who responded "refused," "don't know," "not sure," or "something else" as is common in the literature (Barnes et al. 2014; Blosnich and Bossarte 2012; Chaudhry and Reisner 2019; Scheitle and Wolf 2018). Since I used BMI as a control variable, I dropped females who were pregnant because "BMI is not considered to be appropriate to measure obesity and overweight" for them (Boehmer et al. 2007). I also dropped those under age 25 to appropriately consider the control variable on highest education completed. Weights were not assigned across all four surveys to those who were older than age 45 , so anyone over 45 was also dropped. All analyses were conducted in Stata 14.2 and all descriptive and regression tables can be found in the Appendix.

\section{RESULTS}

Weighted descriptive statistics are presented for females in Tables 2-A to 2-C and for males in Tables 2-H to 2-J in the Appendix. According to Table 2-A, the sexual orientation identity groups for females significantly differ from one another in their sexual attraction, sexual behavior, age, race/ethnicity, nativity, insurance, household income, SRH, USOC, and survey cycle year. There are no significant differences in sexual orientation identity groups by residence, education, or BMI. According to Table 2-B, the sexual attraction groups significantly differ from one another in every demographic, socioeconomic, and SRH, but not for BMI or USOC. The same is true for those who have or have not had same-sex sexual experiences according to Table 2-C.

According to Table $2-\mathrm{H}$, the sexual orientation identity groups for males significantly differ from one another in their sexual attraction, sexual behavior, residence, education, insurance, household income, BMI, USOC, and survey cycle year. There are no significant differences in sexual orientation identity groups by age, race/ethnicity, nativity, or SRH. According to Table 2-I, the male sexual attraction groups significantly differ from one another in sexual identity, sexual behavior, age, residence, education, BMI, USOC, and survey cycle year.

\footnotetext{
${ }^{17} \mathrm{https} / / /$ www-cdc-gov.www.libproxy.wvu.edu/nchs/nsfg/nsfg_combining_data.htm
} 
Males who have or have not had same-sex sexual experiences differ in sexual identity, sexual attraction, race/ethnicity, residence, nativity, education, and all three health-related outcomes according to Table 2-J.

Regarding sexual orientation identity, insurance, household income, USOC, and cycle year are the only non-sexual orientation variables that significantly differ among both females and males; females differ on SRH while males differ on BMI. Age, residence, education, and cycle year are the only non-sexual orientation variables that differ among both females and males when considering sexual attraction. While females differ on SRH, males differ on BMI and USOC. Regarding sexual behavior, both females and males differ on sexual identity, sexual attraction, race/ethnicity, residence, nativity, education, and SRH; males differ on BMI and USOC, but females do not differ on either. Both sets of non-significantly different variables among males and females are significant among the other sex in each corresponding table; that is, there are no variables that are not significant for both sexes in respective tables, they are all either significant in one or both.

There are more males than females in the sample who report either a heterosexual $(95.7 \%$ vs. $91.4 \%$ ) or gay/lesbian (2.5\% vs. $2.2 \%)$ identity, but a greater prevalence of female respondents report being bisexual (6.4\% vs. 1.8\%). Over 10\% $(11.8, \mathrm{CI}=10.8,12.9)$ of heterosexual females report being mostly attracted to males while nearly $100 \%(96.7 \%, \mathrm{CI}=$ $96.2,97.1$ ) of heterosexual males report being only attracted to females; a similar pattern can be seen regarding sexual behavior where $12.7 \%(\mathrm{CI}=11.7,13.7)$ of heterosexual females report same-sex sexual experiences but only $2.8 \%(\mathrm{CI}=2.43 .2)$ of males do. Almost half $(49.4 \%, \mathrm{CI}=$ $44.5,54.4)$ of bisexual females report equal attraction to males and females, but only one-third $(33.7 \%, \mathrm{CI}=24.5,44.3)$ of male bisexuals do. Similarly, over half $(51.2 \%, \mathrm{CI}=47.2,55.1)$ of the females mostly attracted to males report a same-sex sexual experience while only one-third $(32.5 \%, \mathrm{CI}=27.0,38.6)$ of corresponding males do. Of those who have had a sexual experience with a same-sex partner, the majority identify as heterosexual $(64.8 \%, \mathrm{CI}=61.7,67.7$ for females; $44.9 \%, \mathrm{CI}=39.7,50.4$ for males $)$, but more males identify as gay $(34.2 \%, \mathrm{CI}=28.8$, $40.1)$ than bisexual $(20.9 \%, \mathrm{CI}=17.0,25.3)$ while the reverse is seen for females $(10.0 \%, \mathrm{CI}=$ $8.3,12.0$ for gay/lesbian; $25.2 \%, \mathrm{CI}=22.8,27.7$ for bisexual). Only about $10 \%$ of females who have engaged in same-sex behavior are attracted to only or mostly other females while over onethird of males are attracted to only or mostly other males. While attraction to only the opposite 
sex is similar for both sexes among those who have had same-sex sexual experiences, nearly twice as many females report being mostly attracted to males $(37.5 \%, \mathrm{CI}=34.8,40.3)$ than males reporting being mostly attracted to females $(19.7 \%, \mathrm{CI}=15.9,24.1)$.

The younger group has more lesbian/gay and bisexual respondents than the older group for both males and females, but nearly two-thirds $(64.0 \%, \mathrm{CI}=55.5,71.7)$ of female gay/lesbian respondents are in the 25-34 year-old age group while slightly more than half $(53.9 \%, \mathrm{CI}=44.6$, 62.9) of gay males are in the younger group. There appears to be no difference in age groups for heterosexual females, but slightly more heterosexual males in the younger age group. A slightly greater percent of females only attracted to males are in the older group $(52.7 \%, \mathrm{CI}=51.4,54.0$ vs. $47.3 \%, \mathrm{CI}=46.0,48.6$ ), but a much greater percent who are mostly attracted to males are in the younger group $(63.9 \%, \mathrm{CI}=60.5,67.1 \mathrm{vs.} 36.1 \%, \mathrm{CI}=32.9,39.5)$. A larger percent of females mostly attracted to the same sex are in the older group $(52.8 \%, \mathrm{CI}=40.1,65.1 \mathrm{vs}$. $47.2 \%, \mathrm{CI}=34.9,59.9)$, but the opposite is observed for males in this attraction category $(25.8 \%, \mathrm{CI}=16.0,38.9$ vs. $74.2 \%, \mathrm{CI}=61.1,84.0)$. Of those who have engaged in same-sex sexual behavior, more than half are in the younger age group for both males $(53.8 \%, \mathrm{CI}=48.7$, $58.9)$ and females $(57.9 \%, \mathrm{CI}=54.9,60.9)$.

The unweighted descriptive table (Table 2-D) shows significant variation in each healthrelated outcome by sexual orientation component and age for females. Using the dichotomized version of SRH, heterosexual or straight (hereafter: heterosexual) female respondents significantly differ from both homosexual, gay, or lesbian (hereafter: gay/lesbian) and bisexual respondents, as did the full NSFG sample. However, this time this finding is also reflected by sexual attraction. Females who are equally attracted to males and females and those only attracted to the same sex differ from those who are only or mostly attracted to the opposite sex. Comparing the combined underweight and normal categories to the separate overweight and obese categories shows bisexual respondents differ from only heterosexual respondents; females attracted to both sexes differ from all other categories except those attracted to only other females. Access to a USOC only differs between female heterosexual and bisexual respondents, and only attracted to the opposite sex differ from those mostly attracted to males. There are significant differences in SRH and USOC between those who have and have not engaged in same-sex behavior and between the age groups, but only differences in BMI for age group and not by sexual behavior. 
The unweighted descriptive table (Table 2-K) shows significant variation in each healthrelated outcome by sexual orientation component and age for males. There are no differences in the dichotomized version of SRH by sexual orientation identity or by attraction. ${ }^{18}$ Males who identify as gay differ from heterosexual and bisexual males on both SRH and USOC. Those mostly attracted to the same sex and only attracted to the same sex each differ from the other three attraction categories on BMI. Males mostly attracted to the same sex differ from the other categories on USOC and those only attracted to the same sex differ from those only and mostly attracted to the opposite sex. Like the female sample, there are significant differences in SRH and USOC between those who have and have not engaged in same-sex behavior and between the age groups, but only differences in BMI for age group and not by sexual behavior.

In the full models (Tables 2-E to 2-G and 2-L to 2-N) only one set of results shows a significant relationship for both females and males: compared to those who have not had a samesex sexual experience, those who have had sexual experience with a same-sex partner have significantly lower odds of reporting good, very good, or excellent SRH (females OR=0.73, $\mathrm{CI}=0.58,0.91$; males $\mathrm{OR}=0.62, \mathrm{CI}=0.43,0.89$ ). The rest of the results varied by sex.

For females the full model results also show that (a) bisexuals have significantly lower odds of reporting good, very good, or excellent (vs. poor or fair) self-rated health (SRH) ( $\mathrm{OR}=0.62, \mathrm{CI}=0.47,0.82)$ compared to their heterosexual counterparts; and (b) those equally attracted to males and females have significantly lower odds of reporting good, very good, or excellent SRH (OR=0.59, $\mathrm{CI}=0.44,0.81)$ compared to females who are only attracted to the opposite sex. There are no significant differences in relative risks of being overweight or obese by any sexual orientation component for females. Those who are mostly attracted to the opposite sex have significantly lower odds of reporting access to a USOC (OR=0.81, CI $=0.67,0.99$ ) compared to females who are only attracted to the opposite sex.

For males the full model results also show that there are no differences in the odds of reporting good, very good, or excellent SRH by sexual orientation identity or sexual attraction. Compared to males who are heterosexual, those who are gay have significantly lower relative risk of reporting being overweight $(\mathrm{RRR}=0.47, \mathrm{CI}=0.31,0.73)$ or being obese $(\mathrm{RRR}=0.63$, $\mathrm{CI}=0.42,0.95$ ); compared to males only attracted to the opposite sex, those only attracted to the

\footnotetext{
${ }^{18}$ Those mostly attracted to males and equally attracted to males and females are not significantly different according to the Pearson $\chi^{2}$ test or Fisher's exact two-tailed test, but they do differ by the Fisher's exact one-tailed test.
} 
same sex and those mostly attracted to the same sex have significantly lower relative risk of reporting being overweight $(\mathrm{RRR}=0.51, \mathrm{CI}=0.31,0.83 ; \mathrm{RRR}=0.41, \mathrm{CI}=0.20,0.82$, respectively). There are no significant differences in relative risks of being overweight or obese by sexual behavior for males. Compared to heterosexuals, those who are gay or bisexual have significantly higher odds of reporting access to a $\mathrm{USOC}(\mathrm{OR}=2.26, \mathrm{CI}=1.44,3.55 ; \mathrm{OR}=1.64, \mathrm{CI}=1.09,2.47$, respectively); compared to those only attracted to the opposite sex, those who are only attracted to the same sex and those who are mostly attracted to the same sex have significantly higher odds of reporting access to a $\mathrm{USOC}(\mathrm{OR}=1.76, \mathrm{CI}=1.04,2.97 ; \mathrm{OR}=5.37, \mathrm{CI}=2.39,12.08$, respectively); compared to males with no same sex experience, those who have had same sex experiences have significantly higher odds of reporting access to a USOC $(\mathrm{OR}=1.51, \mathrm{CI}=1.14$, 2.01).

Table 2.1. Summary Table of Significant Findings from Female Sample $(\mathrm{N}=12,895)$

\begin{tabular}{|c|c|c|c|c|c|c|c|c|c|c|}
\hline \multirow{3}{*}{ Health Factor } & \multicolumn{10}{|c|}{ Sexual Orientation Component } \\
\hline & \multicolumn{5}{|c|}{ Attraction } & \multicolumn{2}{|c|}{ Behavior } & \multicolumn{3}{|c|}{ Identity } \\
\hline & $\mathbf{O}$ & MO & $\mathbf{E}$ & MS & $\mathbf{S}$ & $\mathbf{N}$ & $\mathbf{Y}$ & $\mathbf{H}$ & $\mathbf{L} / \mathbf{G}$ & $\mathbf{B}$ \\
\hline SRH (excellent-good) & & & - & & & & - & & & - \\
\hline BMI (overweight) & & & & & & & & & & \\
\hline BMI (obese) & & & & & & & & & & \\
\hline USOC (yes) & & - & & & & & & & & \\
\hline
\end{tabular}

Note: $+=$ significant positive association; - = significant negative association; $\mathrm{O}=$ only attracted to the opposite sex; $\mathrm{MO}=$ mostly attracted to the opposite sex; $\mathrm{E}=$ equally attracted to males and females; $\mathrm{MS}=$ mostly attracted to the same sex; $\mathrm{S}=$ only attracted to the same sex; $\mathrm{N}=$ no same-sex behavior; $\mathrm{Y}=\mathrm{yes}$, same-sex behavior; $\mathrm{H}=$ heterosexual or straight; $\mathrm{L} / \mathrm{G}=$ lesbian or gay; $\mathrm{B}=$ bisexual; $\mathrm{SRH}=$ self-rated health (excellent, very good, or good); $\mathrm{BMI}=$ body mass index; USOC=access to usual source of care.

Table 2.2. Summary Table of Significant Findings from Male Sample $(\mathrm{N}=10,672)$

\begin{tabular}{|c|c|c|c|c|c|c|c|c|c|c|}
\hline \multirow{3}{*}{ Health Factor } & \multicolumn{10}{|c|}{ Sexual Orientation Component } \\
\hline & \multicolumn{5}{|c|}{ Attraction } & \multicolumn{2}{|c|}{ Behavior } & \multicolumn{3}{|c|}{ Identity } \\
\hline & $\mathbf{O}$ & MO & $\mathbf{E}$ & MS & $\mathbf{S}$ & $\mathbf{N}$ & $\mathbf{Y}$ & $\mathbf{H}$ & $\mathbf{G}$ & B \\
\hline SRH (excellent-good) & & & & & & & - & & & \\
\hline BMI (overweight) & & & & - & - & & & & - & \\
\hline BMI (obese) & & & & & & & & & - & \\
\hline USOC (yes) & & & & + & + & & + & & + & + \\
\hline
\end{tabular}

Note: $+=$ significant positive association; - = significant negative association; $\mathrm{O}=$ only attracted to the opposite sex; $\mathrm{MO}=$ mostly attracted to the opposite sex; $\mathrm{E}=$ equally attracted to males and females; $\mathrm{MS}=$ mostly attracted to the same sex; $\mathrm{S}=$ only attracted to the same sex; $\mathrm{N}=$ no same-sex behavior; $\mathrm{Y}=\mathrm{yes}$, same-sex behavior; $\mathrm{H}=$ heterosexual or straight; $\mathrm{L} / \mathrm{G}=$ lesbian or gay; $\mathrm{B}=$ bisexual; $\mathrm{SRH}=$ self-rated health (excellent, very good, or good); $\mathrm{BMI}=$ body mass index; $\mathrm{USOC}=$ access to usual source of care. 


\section{DISCUSSION}

These analyses take a finer look at the role sex plays in the relationship between sexual orientation components and SRH, BMI, and access to a usual source of health care. Sexual minority individuals can experience prejudice and discrimination from other statuses such as their sex that can affect health outcomes and access to health-related resources. While this study cannot attest to what types of prejudice and/or discrimination were driving these different results, it does provide more evidence from a nationally representative dataset for the need to further explore them.

Only one set of full model results shows the same significant relationship for both females and males. Males and females who have had sexual experience with a same-sex partner have significantly lower odds of reporting good, very good, or excellent SRH compared to those who have not had a same-sex sexual experience. Sexual attraction and identity also show significant differences in SRH for females, but not for males. The results for females partially align with the findings by Gorman and colleagues (2015) who found bisexuals reporting the highest rates of poor SRH; however, they also found gay men and women reporting the lowest rates of poor SRH while this chapter found no differences for either compared to their heterosexual counterparts. Similarly, Dilley and colleagues (2010) found bisexual women reporting higher odds or poor physical and mental health, but they also found gay men and lesbians reporting the same way and bisexual men reporting higher odds of poor mental health than their heterosexual counterparts. The results in this chapter concur with the findings of Gonzales and colleagues (2016) whereas gay and bisexual men did not differ in the SRH compared to their heterosexual counterparts, but clash with their findings of higher odds of reporting fair or pair SRH among lesbians and no differences in SRH between bisexual women and their heterosexual counterparts.

There were no significant relationships for overweight or obesity by any sexual orientation component for females. This finding is in agreement with one part of the mixed literature on sexual minority female BMI (e.g., Conron et al. 2010), and is particularly helpful as it uses a nationally representative dataset which was not considered in the systematic review by Bowen et al. (2008). However, Boehmer et al. (2007) used the same dataset, albeit in an earlier wave (2002), to find significant differences between lesbians and heterosexual females, but not between bisexuals and heterosexual females. For males, those who are attracted to only or mostly 
the same sex and those who identify as gay had lesser relative risk of being overweight (and obese for gay men) compared to their heterosexual counterparts. These results also follow the literature findings of gay men as statistically significantly less likely to be overweight or obese compared to their heterosexual counterparts with no significant differences between bisexual men and their heterosexual counterparts (Blosnich et al. 2014; Conron et al. 2010).

A review article by Herrick and Duncan (2018) explores the gender difference in physical activity as well as body norms supported by sexual minorities. Mainly focused on gay men and lesbians, it recounts sexual minority men's focus on thinness and muscularity as well as a "fat" stigma which may explain their lower odds of being overweight or obese compared to their sexual majority counterparts found here as well as sexual minority women's acceptance of diverse body shapes that may help explain the non-significant findings here or the significantly higher odds of overweight/obesity seen in other studies (Herrick and Duncan 2018). In a note about bisexual individuals, they point out that the few studies that did consider them found bisexual women at greater risk of "unhealthy physical activity and weight-control behaviors" while bisexual men "did not belong to any profile associated with high levels of physical activity" (Herrick and Duncan 2018:230). Both are consistent with my findings.

Regarding access to a usual source of health care, only females who were mostly attracted to the opposite sex had significantly different (lower) odds of having access compared to their heterosexual counterparts; however, for males all three components of sexual orientation had categories reporting significantly different (all higher) access to care. These results are relatively opposite of what Blosnich and colleagues (2014) found regarding sex and effect direction, but their outcome of interest was actually having a routine check-up. The results of lesbians not differing from their heterosexual counterparts aligns with Conron and colleagues' (2010) findings, but their findings regarding bisexuals (greater odds of no regular provider) and gay men (no difference) is at odds with these results. Dilley and colleagues (2010) also found lesbians and bisexual women not differing from their heterosexual counterparts and found gay men more likely to have a personal doctor than their heterosexual counterparts. They found bisexual men not to differ from their heterosexual counterparts while this chapter found differences by identity, but not by sexual attraction equally to males and females.

Despite providing a more detailed understanding of how sexual orientation and sex intersect and relate to health-related outcomes, this chapter has several weaknesses that need to 
be addressed. First, the NSFG acts within the sex binary and only provides datasets for males and females. It does not ask about gender, so sexual orientation and health variations in sex and gender identity cannot be explored. This limitation forces these analyses to convolute any health outcomes resulting from unique stressors experienced by transgender and non-binary individuals (Matsuno 2019). Future studies should also consider partnership or union status along with sexual orientation and gender, as this can affect SRH differently across groups (Reczek, Liu, and Spiker 2017). A more detailed exploration of racial groups should be conducted as well. Hsieh and Ruther (2016) considered the intersection of sexual identity, race, and gender and found that "conditional on sociodemographic factors, all sexual, gender, and racial minority groups, except straight white women, gay white men, and bisexual non-white men, reported worse self-rated health than straight white men" (746). Another weakness of this chapter is analyzing adults from age 25 to 44 together even though there may be differences in outcomes by age group. Boehmer and colleagues (2007) propose that further investigations into BMI and sexual orientation should consider different age groups which is what Chapter 3 seeks to investigate.

CONCLUSION

Chapter 2 revealed consistent results regarding SRH for females as Chapter 1 did for the whole NSFG sample, but results for males were unique across each health-related outcome. The literature shows that sexual minority males face different pressures when it comes to body image and composition than sexual minority females; these contrasting stressors appear in the BMI results indicating significantly lower relative risk of overweight and obese for gay males. Sexual minority men also have been found to seek various types of health care more often than their heterosexual counterparts, and this was also supported in the NSFG data showing their higher odds of having access to a USOC. Do these findings apply to sexual minorities similarly across a twenty-year age range? Chapter 3 disentangles the role age plays in the relationships between sexual orientation and SRH, BMI, and access to a USOC, and for males and females. 


\section{CHAPTER 3 - EXPLORING HEALTH DISPARITIES BY SEXUAL ATTRACTION, BEHAVIOR, AND IDENTITY BY AGE IN THE UNITED STATES INTRODUCTION}

Fundamental cause theory posits that social conditions - such as race, gender, and socioeconomic status - should be considered when studying health inequities as these factors influence more individually-based health behaviors. These social conditions can be viewed as fundamental causes of health disparities since they put people at risk of other health-related risks. To be designated a fundamental cause, a social factor must influence a variety of health outcomes, affect access to health-related resources, and be associated with health disparities throughout history and across environments. Recent work has proposed stigma as a fundamental cause of health disparities because it shares these characteristics. Minority stress theory discusses the additional stress burden experienced by sexual minorities in various forms of prejudice and discrimination due to their stigmatized group status. Stress alone can lead to health problems, but this excessive stress can help explain the disproportionate rate of health problems experienced by sexual minorities compared to their heterosexual counterparts. Using both of these frameworks, I consider how different components of sexual orientation are associated with two health-related outcomes and access to health-related resources.

I have already explored disparities between sexual orientation components and within them by sex; this chapter considers how age interacts with these relationships. The literature is sparse when it comes to directly comparing age differences in sexual minority health research. Studies have controlled for age, focused on a specific age group, considered how childhood experiences affect later life outcomes, and compared health between different generations, but rarely have they considered age group differences in one study. This chapter uses the National Survey of Family Growth (NSFG; 2011-2019) to expand on the first two chapters and analyze self-rated health (SRH), body mass index (BMI), and access to a usual source of health care (USOC) by two age groups-25-34 year-olds and 35-44 year olds. Comparing 25-34 year-olds with 35-44 year-olds finds only one significant relationship across the two age groups (i.e., lower odds of good SRH for those who have had same sex sexual experiences) and a few different significant relationships between the two groups. The results support the need to consider age and sexual orientation when researching sexual minority health. 


\section{LITERATURE REVIEW}

Age is an important aspect to consider when examining sexual orientation and health. Sexual attraction, behavior, and identity may emerge and evolve at different times across one's life course. While relevant studies often control for age in their analyses, few have exclusively considered age differences in health outcomes for sexual attraction, identity and behavior. SavinWilliams and Vrangalova (2013) explored sexual attraction and identity by age groups (18-24, 25-34, and 34-44) of respondents of the 2002 and 2006-2008 National Survey of Family Growth (NSFG), but did not include any analyses of how they are related to health. Mosher, Chandra, and Jones (2005) explored sexual behaviors by several 5-year age groups with the 2002 NSFG, but did not consider health outcomes by all sexual orientation components and age together, which is what I do here. Exploring age differences in sexual orientation components and their associations with self-rated health, BMI, and access to a usual source of health care provides new insight into the LGB health literature.

Studies that compare multiple age groups are sparse. Research on LGB health tends to focus on either adolescents (e.g., Almeida et al. 2009; Austin et al. 2009; Bontempo and D’Augelli 2002; Coker, Austin, and Schuster 2010; Russell, Driscoll, and Truong 2002), adolescents and young adults (e.g., Brewster and Tillman 2011; $\mathrm{Hu}, \mathrm{Xu}$, and Tornello 2015; Katz-Wise et al. 2014), adults (e.g., Hottes et al. 2016; Solazzo, Gorman, and Denney 2020), or older adults separately (e.g., Brennan-Ing et al. 2014; Fredriksen-Goldsen, Kim, et al. 2013). Recent innovative work by Meyer is looking at differences between generations of sexual minorities. Meyer's project called the Generations study “explores identity, stress, health outcomes, and health care services utilization among LGBs [lesbians, gay men, and bisexuals] in three generations of adults who came of age at different historical contexts" (Anon 2020). Generations differ in their sexual minority identification, with younger generations more often identifying as a sexual minority and using a bisexual identity than older generations (Jones 2021), potentially as a result of decreasing stigma around sexual minorities in general (Gates 2013). However, studies focused on age groups where specific social milestones such as marriage or attending graduate school might be being met are sparse.

More prevalent are studies comparing health effects across the life course. For example, Schneeberger and colleagues (2014) examined studies on stressful childhood experiences (SCE) and their later detrimental health effects on LGBT populations finding a higher prevalence of 
SCE among sexual minorities. Fish and Pasley (2015) used a nationally representative longitudinal survey to explore how attraction, behavior, and identity worked to predict later alcohol use and poor mental health outcomes as adolescents transitioned into adulthood. However, they also note that sexual maturation may happen at different times for different people, particularly for sexual minority youth and that there are some differences by sex (SavinWilliams and Cohen 2007). Sexual maturity and understanding of one's sexual orientation may differ by the two age groups I am examining.

I am once again building off of Boehmer and colleagues' (2007) work which used the 2002 NSFG to explore overweight and obesity via body mass index (BMI) in females. Boehmer and colleagues (2007) included in their article a discussion about the importance of examining age group differences. I use more waves of recent NSFG data of both male and female respondents and am therefore able to stratify my analyses by age group. Although the NSFG does not have data on older adults $(45+)$ for these combined years, exploring potential differences between 25-34 year-olds and 35-44 year-olds still adds new information to the sexual minority health literature.

\section{RESEARCH QUESTION}

1. To what extent do self-rated health, body mass index, and access to a usual source of health care vary by age as well as the three components of sexual orientation-attraction, behavior, and identity—in the United States?

\section{METHODS}

Data

Detailed descriptions of the datasets and measures used from the National Survey of Family Growth (NSFG; 2011-2019) are available in the Data section of this document. Analyses

I produced descriptive statistics by each of the three sexual orientation components separately for those aged 25-34 and those aged 35-44 at the time of the survey. The proportions were weighted to create estimates for the US population of noninstitutionalized men and nonpregnant women aged 25-44 years. I include the $p$-values determined by the "Pearson $\chi^{2}$ test with the Rao and Scott $(1981,1984)$ second-order correction" or the $F$ test (the default output from the Stata svy tabulate command) in these tables that indicate independence between groups 
(stata.com n.d.:11). I also produced an unweighted descriptive table for the adult sample broken up by sexual orientation component and each of the three health-related outcomes. It includes $p$ values determined by Pearson $\chi^{2}$ tests (and Fisher's exact two-tailed tests for $2 \times 2$ tables) comparing subgroups within each status. Percentages are provided for each category for SRH (excellent; very good; good; fair; poor), BMI (underweight, <18.5; normal weight, 18.5-24.9; overweight, 25.0-29.9; obese, >30.0), and USOC (yes; no), but the statistical tests use the dichotomous SRH (excellent, very good, and good; fair and poor) and multinomial BMI (underweight and normal weight, <24.9; overweight, 25.0-29.9; obese, >30.0) outcome categorizations also used in the regressions due to small cell sizes ${ }^{19}$ and examples from pervious literature (e.g., Boehmer et al. 2007; Strutz et al. 2015).

I performed nine logistic regressions that regressed SRH (binomial), BMI (multinomial), and access to a USOC (binomial) on sexual orientation identity, sexual attraction, and sexual behavior separately for those aged 25-34 years and 35-44 years (18 models). Dichotomizing SRH has produced similar results to other methods of analyses (Manor et al. 2000) ${ }^{20}$ and has been used in recent similar work (Solazzo et al. 2020; Strutz et al. 2015). The article which inspired this dissertation analyzed BMI via multinomial logistic regression by combining those who are underweight $(<18.5)$ and normal weight (18.5-24.9) into one category, those who are overweight (25.0-29.9) into another category, and those who are obese (30.0+) into a third category - I took this same approach in my analyses (Boehmer et al. 2007). The question asking respondents whether they had access to a USOC or not only had two non-missing responses (yes and no), so I analyzed it via logistic regression. I controlled for demographic, socioeconomic, and health-related variables.

To prepare the data for analyses, I appended the female respondent data from 2011-2013, 2013-2015, 2015-2017, and 2017-2019 together and appended the male data over the same years together to produce two datasets, one of female respondents and one of male respondents, for the nine-year period. I then appended both sets to each other. Since the NSFG uses a complex sampling design, I also downloaded the corresponding weight files containing the appropriate sample weights and survey design variables. I used the Stata svyset command with the

\footnotetext{
${ }^{19}$ Only 35-44 year-old respondents attracted mostly to the same sex reported less than a high school education. All other cell sizes for each sexual orientation component and variables used in analyses were $\geq 5$.

${ }^{20}$ However, the response they dichotomized was from a SRH variable with four potential values (combined poor and fair, and combined excellent and good).
} 
appropriate weight variable and the corresponding strata and primary sampling unit (psu) variables. Detailed instructions on combining NSFG data can be found at the CDC. ${ }^{21}$

To compare the same samples across tests, I dropped those missing any sexual orientation measures and those who responded "refused," "don't know," "not sure," or "something else" as is common in the literature (Barnes et al. 2014; Blosnich and Bossarte 2012; Chaudhry and Reisner 2019; Scheitle and Wolf 2018). Since I used BMI as a control variable, I dropped females who were pregnant because "BMI is not considered to be appropriate to measure obesity and overweight" for them (Boehmer et al. 2007). I also dropped those under age 25 to appropriately consider the control variable on highest education completed. Weights were not assigned across all four surveys to those who were older than age 45 , so anyone over 45 was also dropped. All analyses were conducted in Stata 14.2 and all descriptive and regression tables can be found in the Appendix. ${ }^{22}$

\section{RESULTS}

Weighted descriptive statistics are presented for 25-34 year-olds in Tables 3-A to 3-C and for 35-44 year-olds in Tables 3-H to 3-J in the Appendix. According to Table 3-A, the sexual orientation identity groups for the younger age group significantly differ from one another in their sexual attraction, sexual behavior, sex, race/ethnicity, nativity, insurance, household income, SRH, BMI, USOC, and survey cycle year-much like the female sample with the exception of BMI. There are no significant differences in sexual orientation identity groups by residence or education. According to Table 3-B, the sexual attraction groups significantly differ from one another in every demographic, socioeconomic, and health-related outcome except residence and household income. Those who have or have not had same-sex sexual experiences differ in every variable except the survey cycle year (Table 3-C).

According to Table 3-H, the sexual orientation identity groups for the older age group significantly differ from one another in their sexual attraction, sexual behavior, sex, residence, nativity, insurance, household income, and SRH. They do not differ by race/ethnicity, education, BMI, USOC, and survey cycle year. According to Table 3-I, the sexual attraction groups significantly differ from one another in every measure except USOC and survey cycle year, and according to Table 3-J those who have or have not had same-sex sexual experiences differ in

\footnotetext{
${ }^{21} \mathrm{https}: / /$ www.cdc.gov/nchs/nsfg/nsfg_combining_data.htm

${ }^{22}$ I have preliminary supplementary analyses by race/ethnicity as well as by both age and sex available upon request; however, there are issues with cell sizes, particular when considering the five categories of sexual attraction.
} 
every variable except residence.

Regarding sexual orientation identity, sex, nativity, insurance, household income, and SRH are the only non-sexual orientation variables that significantly differ among both age groups; only 25-34 year-olds differ on BMI and USOC as well. Sex, race/ethnicity, nativity, education, insurance, SRH, and BMI are the non-sexual orientation variables that differ among both age groups regarding sexual attraction; only the younger group differs by USOC. Regarding sexual behavior, both age groups differ on every variable except residence among the older age group and survey cycle year among the younger age group. Unlike in Chapter two where there are no variables that are not significant for both sexes in corresponding tables, education is not significantly different among either age group by sexual orientation identity, though it does differ for both by sexual attraction and sexual behavior.

A greater percent of respondents in the older sample report a heterosexual identity (94.4\% vs. 92.5\%), but a greater prevalence of younger respondents report being bisexual (5.2\% vs. $3.2 \%) ; 2.3 \%$ of each sample report a gay/lesbian identity. Heterosexual respondents in the younger group are reporting slightly more attraction to mostly the opposite sex $(8.9 \%, \mathrm{CI}=8.1$, $9.8)$ and are less so in the older group $(5.6 \%, \mathrm{CI}=5.0,6.4)$, but they are more similar in reporting same-sex sexual behavior $(8.0 \%, \mathrm{CI}=7.3,8.7$ for $25-34$ year-olds; $7.1 \%, \mathrm{CI}=6.3,7.9$ for 35-44 year-olds). A greater proportion of bisexuals in the older group $(87.1 \%, \mathrm{CI}=81.8$, 91.1) report engaging in same-sex behavior than in the younger group $(80.5 \%, 74.8,85.2)$; however, of those who have engaged in same-sex behavior more report a bisexual identity among the younger group $(26.9 \%, 24.3,29.7)$ than the older group $(20.4 \%, \mathrm{CI}=17.5,23.6)$. Those reporting equal attraction to males and females in the younger group $(76.3 \%, \mathrm{CI}=69.5$, 82.1) have a smaller proportion reporting same-sex sexual experience than in the older group $(84.7 \%, \mathrm{CI}=76.9,90.2)$. Of the $35-44$ year-olds mostly attracted to the opposite sex, they are about split regarding same-sex sexual experiences, but in the 25-34 year-old group, there is about a 10 percentage points greater difference in reporting no sexual experience with a same-sex partner $(54.8 \%, \mathrm{CI}=50.9,58.6$ vs. $45.2 \%, \mathrm{CI}=41.4,49.1)$. In the older group $42.2 \%(\mathrm{CI}=38.7$, 45.9) of them who have engaged in same-sex behavior are attracted to only the opposite sex while only $31.5 \%(\mathrm{CI}=28.4,34.7)$ of respondents in the younger group report that attraction.

Both age groups have roughly the same proportion of male and female bisexual respondents, but there are a greater proportion of females reporting a gay/lesbian identity in the 
younger group $(51.2 \%, \mathrm{CI}=42.0,60.2$ females $)$ and a lesser proportion in the older group $(40.8 \%, \mathrm{CI}=32.4,49.7$ females). Sex distribution is about split in the older group among heterosexual respondents, but there are more male $(53.2 \%, \mathrm{CI}=51.8,54.5)$ than female heterosexuals in the younger group. In the older age group, there are $68.4 \%(\mathrm{CI}=55.8,78.8)$ of those mostly attracted to the same sex who are female, but only $40.3 \%(\mathrm{CI}=27.8,54.2)$ in the younger age group. In the older age group, only $31.2 \%(\mathrm{CI}=23.6,40.0)$ of those only attracted to the same sex are female, but $54.9 \%(\mathrm{CI}=44.5,64.9)$ in the younger age group are female. Of those who have had a sexual experience with a same-sex partner, there is a greater gap between males and females in the younger group $(76.0 \%, \mathrm{CI}=72.8,78.9$ female vs. $24.0, \mathrm{CI}=21.1,27.2$ male) than in the older group $(72.8 \%, \mathrm{CI}=69.1,76.3$ female vs. $27.2, \mathrm{CI}=23.7,30.9$ male $)$.

The unweighted descriptive table (Table 3-D) shows significant variation in each healthrelated outcome by sexual orientation component and sex for the younger 25-34 year-old age group. Bisexual respondents significantly differ from both gay/lesbian and heterosexual respondents on the dichotomized version of SRH. This finding is reflected by sexual attraction where those equally attracted to males and females differ from those only or mostly attracted to the opposite sex and those only attracted to the same sex. Comparing the combined underweight and normal categories to the separate overweight and obese categories again shows bisexual respondents differ from gay/lesbian and heterosexual respondents; respondents attracted to both sexes differ from all other categories. Those mostly attracted to the opposite sex also differ from those only attracted to the same sex on all three health-related outcomes. Heterosexual respondents differ from both gay/lesbian and bisexual respondents on access to a USOC. Those only attracted to the opposite sex differ from all other categories except those only attracted to the same sex. Those mostly attracted to the same sex differ from all other categories except those equally attracted to males and females. There are significant differences in SRH, BMI, and USOC between those who have and have not engaged in same-sex behavior and between males and females.

The unweighted descriptive table (Table 3-K) shows significant variation in each healthrelated outcome by sexual orientation component and sex for the older 35-44 year-old age group. SRH is the only health-related outcome that has differences by sexual orientation identity (heterosexual respondents differ from both gay/lesbian and bisexual respondents). As expected, those only attracted to the opposite sex then differ in SRH between those equally attracted to 
males and females and those only attracted to the same sex. Those mostly attracted to the opposite sex also differ in SRH from those equally attracted to males and females. Those equally attracted to males and females differ from those only attracted to the opposite or same sex in BMI. Those only attracted to the opposite sex differ from those mostly attracted to the opposite sex in BMI and are the only two attraction groups different in USOC. Like the younger sample, there are significant differences in SRH, BMI, and USOC between those who have and have not engaged in same-sex behavior and between males and females.

In the full models, only one set of results shows a significant relationship for both those aged 25-34 years and those aged 35-44 years: compared to those who have not had a same-sex sexual experience, those who have had sexual experience with a same-sex partner have significantly lower odds of reporting good, very good, or excellent $\mathrm{SRH}(\mathrm{OR}=0.65, \mathrm{CI}=0.50$, $0.84 ; \mathrm{OR}=0.74, \mathrm{CI}=0.55,0.99$, respectively). The rest of the results varied by age group.

For the 25-34 age group the full model results also show that (a) bisexuals have significantly lower odds of reporting good, very good, or excellent (vs. poor or fair) self-rated health $(\mathrm{SRH})(\mathrm{OR}=0.56, \mathrm{CI}=0.42,0.76)$ compared to their heterosexual counterparts; and (b) those equally attracted to males and females have significantly lower odds of reporting good, very good, or excellent $\mathrm{SRH}(\mathrm{OR}=0.54, \mathrm{CI}=0.39,0.74)$ compared to those who are only attracted to the opposite sex. There are no significant differences in relative risks of being overweight by any sexual orientation component. Bisexuals have higher odds of reporting being obese $(\mathrm{OR}=1.41, \mathrm{CI}=1.06,1.87)$ compared to their heterosexual counterparts. Compared to heterosexuals, lesbian/gay respondents have significantly higher odds of reporting access to a USOC (OR=1.57, $\mathrm{CI}=1.06,2.34)$; compared to those only attracted to the opposite sex, those who are mostly attracted to the same sex have significantly higher odds of reporting access to a $\mathrm{USOC}(\mathrm{OR}=3.20, \mathrm{CI}=1.44,7.11)$.

For the 35-44 age group, only one other full model has significant results. Those mostly attracted to the opposite sex have significantly lower relative risk of being overweight compared to those only attracted to the opposite sex $(\mathrm{OR}=0.76, \mathrm{CI}=0.55,0.94)$. 
Table 3.1. Summary Table of Significant Findings from Sample Aged 25-34 (N=13,038)

\begin{tabular}{|c|c|c|c|c|c|c|c|c|c|c|}
\hline \multirow{3}{*}{ Health Factor } & \multicolumn{10}{|c|}{ Sexual Orientation Component } \\
\hline & \multicolumn{5}{|c|}{ Attraction } & \multicolumn{2}{|c|}{ Behavior } & \multicolumn{3}{|c|}{ Identity } \\
\hline & $\mathbf{O}$ & MO & $\mathbf{E}$ & MS & $\mathbf{S}$ & $\mathbf{N}$ & $\mathbf{Y}$ & $\mathbf{H}$ & $\mathbf{L} / \mathbf{G}$ & B \\
\hline SRH (excellent-good) & & & - & & & & - & & & - \\
\hline BMI (overweight) & & & & & & & & & & \\
\hline BMI (obese) & & & & & & & & & & + \\
\hline USOC (yes) & & & & + & & & & & + & \\
\hline
\end{tabular}

Note: $+=$ significant positive association; - = significant negative association; $\mathrm{O}=$ only attracted to the opposite sex; $\mathrm{MO}=$ mostly attracted to the opposite sex; $\mathrm{E}=$ equally attracted to males and females; $\mathrm{MS}=$ mostly attracted to the same sex; $\mathrm{S}=$ only attracted to the same sex; $\mathrm{N}=$ no same-sex behavior; $\mathrm{Y}=\mathrm{yes}$, same-sex behavior; $\mathrm{H}=$ heterosexual or straight; $\mathrm{L} / \mathrm{G}=$ lesbian or gay; $\mathrm{B}=$ bisexual; $\mathrm{SRH}=$ self-rated health (excellent, very good, or good); $\mathrm{BMI}=$ body mass index; $\mathrm{USOC}=$ access to usual source of care.

Table 3.2. Summary Table of Significant Findings from Sample Aged 35-44 (N=10,529)

\begin{tabular}{|l|c|c|c|c|c|c|c|c|c|c|}
\hline \multirow{2}{*}{ Health Factor } & \multicolumn{9}{c|}{ Attraction } & \multicolumn{3}{c|}{ Bexual Orientation Component } \\
\hline & O & MO & E & MS & S & N & Y & H & G/L & B \\
\hline SRH (excellent-good) & & & & & & & - & & & \\
\hline BMI (overweight) & & - & & & & & & & & \\
\hline BMI (obese) & & & & & & & & & & \\
\hline USOC (yes) & & & & & & & & & & \\
\hline
\end{tabular}

Note: $+=$ significant positive association; - = significant negative association; $\mathrm{O}=$ only attracted to the opposite sex; $\mathrm{MO}=$ mostly attracted to the opposite sex; $\mathrm{E}=$ equally attracted to males and females; $\mathrm{MS}=$ mostly attracted to the same sex; $\mathrm{S}=$ only attracted to the same sex; $\mathrm{N}=$ no same-sex behavior; $\mathrm{Y}=\mathrm{yes}$, same-sex behavior; $\mathrm{H}=$ heterosexual or straight; $\mathrm{L} / \mathrm{G}=$ lesbian or gay; $\mathrm{B}=$ bisexual; $\mathrm{SRH}=$ self-rated health (excellent, very good, or good); $\mathrm{BMI}=$ body mass index; USOC=access to usual source of care.

\section{DISCUSSION}

These analyses separate adults by age groups (25-34 and 35-44 years-old) to look at the role age plays in the relationship between sexual orientation and SRH, BMI, and access to a USOC. The article inspiring this dissertation (i.e., Boehmer et al. 2007) called for scholars to produce studies that could focus on age differences in BMI since their sample was too small and previous related research used samples with older average ages. While this study remains within roughly the same age-range as Boehmer and colleagues (2007) since it uses the same survey, albeit with more recent data over a longer period of time, I am able to separate the respondents into two age groups for analyses. Doing so has provided results showing differences in significance on several outcomes depending on age group.

According to summary tables in Chapter 1, of the respondents who identified as bisexual, $65.3 \%(\mathrm{CI}=61.3,69.2)$ of them were aged $25-34$ while only $34.7 \%(\mathrm{CI}=30.8,38.7)$ were aged 35-44 years-old. Other sexual minority health studies have found a large portion of their bisexual 
respondents to have poorer health despite being younger compared to other identities (Conron et al. 2010; Dilley et al. 2010; Gorman et al. 2015). Recent results from a Gallup poll show that over $70 \%$ of Generation Z (born 1997-2002) adults who identify as a sexual minority identify as bisexual while only about half of Millennials (born 1981-1996) do; there is no discernible difference between identifying as bisexual or gay/lesbian in older generations (Jones 2021). Though only Millennials and Generation X (born 1965-1980) are considered in these chapters' analyses (i.e., age 25-44 between 2011 and 2019), there is a clear pattern of increased bisexual identification with younger respondents. One potential explanation is that stigma is declining around the bisexual identity so more people feel comfortable using it (Gates 2013). Despite potential biphobia from both heterosexuals and other sexual minorities (Callis 2013) which may be negatively affecting health, bisexuality has been gaining traction in both media (Townsend 2019) and identity use (Jones 2021).

Only one set of full model results shows the same significant relationship for both age groups. The 25-34 year-old age group and 35-44 year-old age group respondents who have had sexual experience with a same-sex partner have significantly lower odds of reporting good, very good, or excellent SRH compared to those who have not had a same-sex sexual experience. Only one other response category across the three sexual orientation components showed a significant difference from their majority group counterparts in the older age group, and that was those who were mostly attracted to the opposite sex had a lower relative risk of being overweight compared to those only attracted to the opposite sex. More differences emerged across sexual orientation component and outcome in the younger age group.

Sexual behavior was the only sexual orientation component among the older group that had significant differences in SRH, but in the younger age group those who identified as bisexual and those who were attracted equally to females and males had lower odds of reporting good, very good, or excellent SRH compared to their heterosexual and only opposite sex attracted counterparts. The results from the younger group are similar to the findings of Conron and colleagues (2010) of bisexuals having higher odds of reporting poor/fair SRH in their 18-64 year-old sample. Strutz and colleagues (2015) examined a 24-32 year-old sample and found a difference in SRH by gender; a greater percent of sexual minority women reported poor/fair SRH while there were no differences between sexual minority men and their heterosexual counterparts. Fredriksen-Goldsen and colleagues (2013) examined a sample of adults aged 50 
and over from a 2003-2010 Washington State BRFSS and found higher odds of poor mental health for sexual minority men and women and higher odds of poor physical health for sexual minority men compared to their heterosexual counterparts. The authors suggested their findings show persistent poorer health behaviors and outcomes for sexual minorities as seen in other studies of younger samples, but the 35-44 year-olds in this chapter do not follow this pattern.

The only differences that emerged related to BMI were that those who identified as bisexual had a higher relative risk of being obese than their heterosexual counterparts in the younger group and those who were mostly attracted to the opposite sex had a lower relative risk of being overweight than their counterparts only attracted to the opposite sex in the older group. These findings add to the mixed literature on overweight and obesity among sexual minorities; however, the non-significant findings might be masking some gender differences in outcomes. For example, Fredriksen-Goldsen and colleagues (2013) found higher odds of obesity for sexual minority women, and lower odds of obesity for sexual minority men as did Conron and colleagues (2010) for lesbians and gay men, respectively. Herrick and Duncan (2018) point out age differences in perceptions of diverse body shapes among sexual minority women. They found younger sexual minority women were more accepting of diverse body types, however as they aged they began to more deeply consider the adverse health effects of being overweight or obese (Herrick and Duncan 2018). This could be reflected in my supplementary findings of higher relative risk of obesity among 25-34 year-old bisexual females and no significant difference between 35-44 year-old bisexual females and their heterosexual counterparts.

Fredriksen-Goldsen and colleagues (2013) did have one finding align with this chapter's results from the older group - they found no differences on access to health care. There were no differences among any sexual orientation status in the older group compared to their sexual orientation majority counterparts. In the young group, however, those who were mostly attracted to the same sex or identified as gay/lesbian had higher odds of having access to a USOC. In my supplementary analyses, it appears it is the younger males driving these results. Gay men have been found to utilized mental health care services more often than their heterosexual male counterparts (Platt et al. 2018) as well as participating in some more preventive health behavior and screening tests, but there is no difference between them and getting a routine check-up (Blosnich et al. 2014). Since the USOC question in the NSFG asks if respondents have a place they usually go to when sick or needing advice about health, male respondents may be referring 
more towards use of mental health or preventative services in their answers than routine checkups.

Several limitations of these analyses must be noted as well. These two 10-year age groups (25-34 years-old and 35-44 years-old) may not be the best way to group ages together (e.g., 5-year age groups may be more appropriate). Another limitation is that two age groups compared here are restricted by the age of the population the survey targets. Boehmer and colleagues (2007) point out a 2001 Surgeon General's report (US Department of Health \& Human Services 2001) that cites Eberhardt, Ingram, and Makuc (2001) saying overweight and obesity can increase until age 60 before a decrease can be seen; however, a more recent piece from the Office of the Surgeon General (US) (2010) says "At any stage of life, increased consumption of excess calories from fats and added sugars in foods that are energy dense, such as fast food, is associated with obesity" (4). While I could have included younger participants (to age 15), the CDC (2020) notes that BMI is not interpreted the same way for those under 20 years-old. Finally, these analyses do not account for period or cohort effects. Future research can address these limitations and contribute to the discussion on age and sexual minority health. CONCLUSION

Chapter 3 revealed consistent results regarding SRH and for 25-34 year-olds as Chapter 1 did for the whole NSFG sample and Chapter 2 did for females, but results for those age 35-44 years were mostly insignificant sexual orientation measures. Bisexuals in the younger group also had higher relative risk of being obese while lesbian/gay respondents and those who were mostly attracted to the same sex had higher odds of having access to a USOC. Those who were mostly attracted to the opposite sex in the older group had significantly lower relative risk of being overweight than their counterparts only attracted to the opposite sex. The literature specifically comparing age differences in sexual minority health is quite nascent, so these results add a unique perspective to the overall narrative while using a nationally representative sample. Future research can use the findings from these three chapters to explore other intersectional statuses, such as race/ethnicity as I did in preliminary supplemental analyses, in greater detail and with contextual variables to more clearly elucidate these results. 


\section{DISCUSSION}

Most previous sexual minority health research has focused on mental health and substance use. The three health-related variables explored in this dissertation represent a subjective holistic health outcome (i.e., SRH), an objective physical health outcome (i.e., BMI) and access to a health-related resource (i.e., access to a USOC). Below I discuss the findings pertaining to each health-related outcome, how they function within the overall sample and each subsample, and how the results fit with the current sexual minority health literature. I also consider how they correspond to the fundamental cause and minority stress frameworks and why lack of contextual data may have contributed to some of the more enigmatic results.

One main goal of this dissertation was to consider how different group statuses intersected with sexual orientation. Chapter 2 explored how health-related outcomes were affected by sexual orientation components and sex, Chapter 3 explored them by sexual orientation components and age, and preliminary supplemental analyses explored them by sexual orientation components and race as well as by sex and age together. Intersectionality research of this kind is fairly new due to sampling and other methodological limitations of previous surveys (Tuthill, Denney, and Gorman 2020). This study uses a large sample of recent (i.e., 2011-2019) nationally representative data with multiple questions about sexual orientation and health to add to the sexual minority health literature.

When considering the intersectionality of these social statuses on health-related outcomes, it is important to note that it is not the statuses themselves that are driving health disparities, but rather the structural and cultural response to members of these social groups. Being a sexual, gender, or racial/ethnic minority in and of itself does not necessarily cause poor health, but rather the experiences of prejudice and discrimination by other people and institutions/systems leads to excess stress and consequently poorer health (Meyer 2003). Stigmatized groups are at increased risk of risks with less access to resources to improve or maintain health or to mitigate disease (Hatzenbuehler et al. 2013; Link and Phelan 1995). Heterosexism, homophobia, biphobia, sexism, ageism, and racism are all examples of prejudice and discrimination against historically oppressed minority groups that can negatively affect their health, creating gaps in their quality of life between them and their corresponding majority group members. 


\section{SELF-RATED HEALTH}

I chose to explore variations in SRH because of its close association with morbidities (Farmer and Ferraro 1997; Ferraro et al. 1997; Ferraro and Su 2000; Idler and Kasl 1995) and mortality (Jylhä 2009; Schnittker and Bacak 2014; Stoddard et al. 2019) as well as its prevalence of use in studying the sociology of health (Jylhä 2009). SRH has also been used in sexual minority health literature, so I can compare my findings across studies (Conron et al. 2010; Gorman et al. 2015; Strutz et al. 2015). I found negative reports of SRH in at least one sexual minority orientation component status within the main sample and every subsample. This troubling trend shows that sexual minorities have higher odds of perceiving their own health as poor or very poor. Although the analyses of BMI and access to a USOC yielded mainly nonfindings and even some potentially favorable results, the consistently negative SRH is alarming due to its holistic attribute and close relation to other morbidities and mortality. Persistent poor SRH indicates an overall negative health experience that could indicate those who may need some type of health-related or medical intervention.

In the overall NSFG sample, those who are equally attracted to males and females, those who have had same sex experiences, and those who identify as bisexual all have lower odds of reporting very good, good, or fair health compared to their sexual majority counterparts. No subsample reports the same for gay or lesbian respondents or those who are mostly or only attracted to the same sex. ${ }^{23}$ Every subsample reports poorer SRH for those who have had same sex experiences, and about half of the subsamples report poorer SRH for those who are equally attracted to males and females. No subsample sexual orientation component value reports higher SRH compared to their heterosexual counterpart. These findings illustrate the need to further investigate what is driving poor SRH among bisexuals and those who have same sex experiences.

A likely explanation for higher poor SRH among bisexuals would be their experiences of biphobia. They are at risk of experiencing biphobia from both heterosexuals and within the sexual minority community. Attitudes towards gay men, lesbians, and bisexuals has been found to differ with bisexuals bearing a greater prejudice from others for being "hypersexual" or accused of using a temporary identity until one picks either gay or straight (Callis 2013; Worthen

\footnotetext{
${ }^{23}$ My preliminary supplementary analyses found females aged 35-44 years only attracted to the same sex also had poorer SRH, but should be considered with caution due to multiple small cell sizes.
} 
2013). Previously discussed literature has shown that bisexuals tend to be at greater risk of poor health behaviors and outcomes than their heterosexual and their gay and lesbian counterparts (Gorman et al. 2015). Their article suggested poorer socioeconomic status among bisexuals was driving their poorer SRH (Gorman et al. 2015), a notion also found in other studies (Conron et al. 2010; Dilley et al. 2010) and partially supported here as well.

The lack of significant difference in SRH between gay and lesbian respondents and their heterosexual counterparts appears counterintuitive, but recent research using BRFSS data from seven states has shown similar results. After adjusting for a host of sociodemographic, social network, and health behavior variables lesbians had a significantly lower predicted probability of poor SRH compared to heterosexual men and no difference compared to heterosexual women; gay men had no difference compared to heterosexual men or women (Gorman et al. 2015). Strutz and colleagues (2015) examined the National Longitudinal Study of Adolescent to Adult Health (Add Health) data when respondents were 25-35 years-old and also found no differences in SRH between various measures of sexual minority men and their heterosexual counterparts, but found sexual minority women had worse SRH than their heterosexual counterparts. Data from the 2013-2014 National Health Interview Surveys (NHIS) also showed lesbians had higher odds of reporting fair or poor SRH than their heterosexual counterparts, but no differences between gay men, bisexual men, or bisexual women and their heterosexual counterparts (Gonzales et al. 2016). Continued research in this area, with an additional focus on context, is needed to understand these varied results.

Differences in SRH between sexes by sexual orientation are also avenues ripe for exploration. In their adjusted models, Gorman and colleagues (2015) found heterosexual men had a significantly higher predicted probability of poor SRH compared to heterosexual women, but no gender differences were found between gay men and lesbians or between bisexual men and bisexual women. Data from a single-state BRFSS found gay men, lesbians, and bisexual women each had higher odds of reporting poor physical and mental health than their heterosexual counterparts while bisexual men had higher odds of only reporting poorer mental health than their heterosexual counterparts (Dilley et al. 2010). While I did not directly compare gay men to lesbians and bisexual men to bisexual women, I found that within the female sample the same significant poorer SRH among bisexual women compared to their heterosexual counterparts, but no difference in SRH between bisexual men and their heterosexual 
counterparts. Perhaps bisexual men are giving more weight to their physical health than their mental health in responding to a question about general health.

I found the same pattern in the 25-34 year-old group as I did in the overall sample and female sample, and found the same pattern in the 35-44 year-old group as I did in the male sample. A potential explanation for the discrepancies may be because the younger group was made up of more respondents who identified as bisexual. This trend follows a national Gallup poll which found younger generations more likely to use a bisexual identity than older ones (Jones 2021). Of the respondents who identified as bisexual in the full NSFG sample, 77\% $(\mathrm{CI}=73.8,86.2)$ are female while $22.3 \%(\mathrm{CI}=18.8,26.2)$ are male. This finding also aligns with the recent Gallup Poll (Jones 2021). Poor SRH in younger bisexual females may be what is showing through in the national SRH patterns.

\section{BODY MASS INDEX}

Body mass index (BMI) results were the least consistent across samples. Few significant differences emerged across any of the samples by sexual orientation component. The results from Chapter 1 show that bisexuals have higher relative risk of reporting a BMI that categorizes them as obese compared to their heterosexual counterparts. However, when examined in Chapter 2 and 3 subsamples, only the 25-34 year-old age group produced the same results. No other minority sexual orientation component status had higher relative risk of overweight or obesity compared to their majority counterparts. ${ }^{24}$ In the Chapter 2 male subsample I found those who were attracted to mostly the same sex or only the same sex had lower relative risk of reporting being overweight compared to their counterparts only attracted to the opposite sex. Those who identified as gay had lower relative risk of reporting overweight and obesity compared to their heterosexual counterparts. Curiously, those who reported attraction mostly to the opposite sex in the 35-44 year-old age group also had lower relative risk of being overweight compared to their counterparts. These findings add to the mixed literature on the topic for women and concur with the patterns typically seen among men.

While some studies have found evidence of higher BMI among sexual minority women, others have found no differences between them and their sexual majority counterparts. A review article on obesity issues in sexual minority women says that nine of 19 relevant studies found

\footnotetext{
${ }^{24}$ My preliminary supplementary analyses reveals higher relative risk of obesity and overweight for some groups, but should be considered with caution due to multiple small cell sizes.
} 
evidence of "higher weight or obesity rates among lesbians than control heterosexual samples," but five of the studies found no differences and the remaining four did not have comparison groups (Bowen et al. 2008:226). While they also noted that none of the studies were nationally representative and that the verdict on exercise levels between sexual minority and majority women was uncertain, they still concluded that obesity was an issue for sexual minority women (Bowen et al. 2008). Two later studies, one using 2002 NSFG data and the other using 20012006 Massachusetts BRFSS data, found lesbians were more likely to be overweight and obese compared to their heterosexual counterparts, but did not find differences between bisexual women and their heterosexual counterparts (Boehmer et al. 2007; Conron, Mimiaga, and Landers 2008). Kinsky (2015) lists several other state-level studies that found lesbians had higher odds of overweight or obesity compared to their heterosexual counterparts. On the other hand, Katz-Wise and colleagues (2014) used data from the US National Longitudinal Study of Adolescent Health (ages 11-34 years) to find that Latina and White bisexual women had higher BMIs than their heterosexual counterparts across all ages analyzed, but no differences for lesbians. Race/ethnicity should be further investigated when considering sexual minority health as Molina and colleagues (2013) found African American lesbians and bisexual women had a higher average BMI than White lesbians and bisexual women, though both averages were greater than the normal BMI range.

There are several ideas to consider when trying to account for these discrepancies. Bowen and colleagues (2008) note that there were flaws with the methodologies of the studies they reviewed (e.g., some lacked heterosexual control groups, most used convenience samples) and there was an overall inconsistency in the measurements of sexual orientation across studies. These studies have used samples from different locations and the ages of the samples also vary. While the findings are not completely consistent, I have not come across any studies finding sexual minority women as being less likely to be overweight or obese than their sexual majority counterparts. Healthy People 2020 (2020) note elevated overweight and obesity among lesbians and bisexuals as a health disparity that needs to be addressed by health care and public health professionals. Weight is a factor that should continue to be investigated among sexual minority women, especially given obesity's tie to multiple adverse health outcomes (Daniels et al. 2005; Fredriksen-Goldsen, Emlet, et al. 2013; Solomon and Manson 1997), but it should not be the only measurement explored as there are better methods to measure body fat (Nuttall 2015). 
Several studies contend that lesbians are at greater risk of being overweight or obese compared to heterosexual women and seek to understand why. For example, Mereish (2014) found among a nonprobability sample of lesbians with an average BMI in the overweight category that those who had experienced heterosexist discrimination had higher odds of being overweight and obese compared to lesbians with normal BMIs. This finding aligns with minority stress theory and considering stigma as a fundamental cause of health disparities. Conversely, Molina and colleagues (2013) found no relationship between internalized sexism and BMI among lesbian and bisexual women, but did find it associated with lower levels of physical activity. They suggest that cultural norms negatively view participation in exercise or athletics as masculine, thus women with internalized sexism may decide not to engage in such activities (Molina et al. 2013). Relationship status, depressive symptoms, and binge drinking may also play a role in overweight and obesity among partnered lesbians (Mason and Lewis 2015). Qualitative work using focus groups has found a lack of consensus among lesbians as to their attitudes towards weight and BMI (Roberts, Stuart-Shor, and Oppenheimer 2010). As more nationally representative and contextual data become available, researchers should continue to investigate the prevalence of overweight and obesity among sexual minority women and potential driving mechanisms.

Research exploring the weight status of gay men has been more consistent in finding them less likely to be overweight or obese than their heterosexual counterparts, though findings for bisexual men have varied. For example, Newlin Lew and colleagues (2018) used the Behavioral Risk Factor Surveillance System (BRFSS) data from 19 states in the US to find gay men had lower odds for obesity; however, they did find bisexual men had higher odds of diabetes than their heterosexual counterparts. Katz-Wise and colleagues (2014) found heterosexual male respondents had greater one-year gains in BMI than gay males across all three races/ethnicities analyzed, but non-Latino White male bisexuals had greater one-year BMI gains than their heterosexual counterparts. Conron, Mimiaga, and Landers (2008) found gay men were less likely to be overweight or obese compared to their heterosexual counterparts, but found no differences between bisexual men and their heterosexual counterparts. Deputy and Boehmer (2010) found when grouped together, gay and bisexual men had lower BMIs compared to their heterosexual counterparts, though their sample had three times as many gay men than bisexual men. 
These patterns may reflect literature finding gay men may put greater emphasis on physical appearance in attempting to attract a male partner, similar to the way heterosexual women may do (Wood 2004) as well as the media-driven existence of a "certain ideal body type" that is "'fit", 'masculine' and 'muscular"' (Brennan et al. 2013). Compared to heterosexual men, gay men report more body shape dissatisfaction which for them was also more strongly correlated with eating disorder symptomatology, drive for thinness, and self-esteem (Yean et al. 2013). Deputy and Boehmer (2010) did not find evidence of diet and exercise mediating the relationship between sexual orientation and BMI using the population-based 2006 California Health Interview Survey, instead, they found that sexual orientation, diet, and exercise each had an independent effect on BMI. While research may find sexual minority men less likely to be overweight or obese than their sexual majority counterparts, it does not necessarily mean they are healthier - public health and health care professionals should consider the negative coping behaviors and mental toll they may experience trying to achieve idealized body types.

Overall, I find BMI is generally higher for bisexuals and is lower for gay men and males attracted to the same or mostly same sex. Obesity among bisexuals may be a result of poorer socioeconomic statuses and excess stigmatization and discrimination from heterosexuals, gay men, and lesbians. Lower BMI among sexual minority men may be driven by a cultural body type ideal that promotes a thinner muscular body composition; however, striving to reach this ideal may come at the expense of their mental health. Although obesity is associated with a variety of adverse health outcomes, BMI is not necessarily the optimal measurement for body fat, so future surveys need to gather data on multiple physical health indicators. Studies should also consider mental and physical health together to gain a better understanding of sexual minority health in general.

\section{ACCESS TO A USUAL SOURCE OF HEALTH CARE}

The NSFG's question about access to a USOC asks if there is a place that the respondent usually goes to when they are sick or need advice about health. When examining the whole NSFG sample, no significant differences in access to a USOC emerges across any sexual orientation status. However, when broken down by sex, we see higher odds of access to a USOC among most male sexual orientation minority statuses. Among females, those who are mostly attracted to the opposite sex actually have lower odds compared to those only attracted to the opposite sex of having a USOC. When separated by age, younger participants who are mostly 
attracted to the same sex or identify as gay/lesbian have higher odds of access to a USOC while no differences are found in the older age group. Overall, findings appear encouraging, but given previous literature around access to health care for sexual minorities, I will discuss why this should be interpreted cautiously.

Higher odds of USOC for males who are attracted to mostly and only the same sex, who have had same sex sexual experiences, and who identify as either gay or bisexual compared to their sexual orientation majority counterparts is not an unexpected finding. For example, Platt, Wolf, and Scheitle (2018) found gay and bisexual men more likely to have seen a mental health professional in the past year than their heterosexual counterparts. Blosnich and colleagues (2014) found gay men had higher odds of participating in some more preventive health behavior and screening tests, but no difference between them and getting a routine check-up; they also found bisexual men only more likely to get one type of screening test (at a lower odds than gay men) compared to their heterosexual counterparts. Considering the wording of the NSFG question on USOC, it is possible that male respondents may be reflecting on their use of mental health or preventative services in their answers instead of annual exams.

The lack of significant differences in access to a USOC among females is a bit more complex. Blosnich and colleagues (2014) found no differences between lesbians or bisexual women and their heterosexual counterparts regarding any preventative health behaviors or screening tests when controlling for other factors, but did find lesbians had lower odds of a routine check-up and bisexual women had higher odds of not seeking care due to cost compared to their heterosexual counterparts. These findings align with mine that show no differences in access to a USOC and potentially bisexuals' higher rates of poor SES. They also lend support to the notion that respondents were not thinking of a routine exam when answering this question. However, Platt and colleagues (2018) found sexual minority women more likely to have seen a mental health professional recently than their heterosexual counterparts, but they did not differ from their male sexual minority counterparts in utilization. The USOC question may not be evoking thoughts of being "sick" mentally and seeking professional help, but rather conjuring up ideas of physical sickness or prevention.

When explored by age, only the younger group reported any significant differences in access to a USOC. Those who were mostly attracted to the same sex or identified as gay/lesbian had higher odds of a USOC. The lack of differences in the older group is somewhat unexpected, 
particularly because the average ages of the respondents in Blosnich and colleagues' (2014) paper would have put them in that age category. The mean age of the respondents in Platt and colleagues' (2018) article was almost 47 years-old.

The USOC question itself does not ask about the quality of health care received, so it is possible there are qualitative differences in the experiences of sexual minorities and sexual majorities at their USOC locations. It is also possible that some respondents considered the internet as a "place" they could go to for "advice about health" which may explain the generally high response rate across samples saying they did have a USOC. More specific questions about quality of health care experiences, reasons for visits, and frequency of visits could provide a clearer picture as to the health care access of sexual minorities.

\section{THEORETICAL IMPLICATIONS}

Although not every minority social orientation status reported poorer health outcomes compared to their majority group counterparts, these analyses are only a piece of the puzzle and do not suggest that fundamental cause theory and/or minority stress theory are not at work. A nationally representative study is helpful for seeing patterns across the country, but it does not reveal patterns within the county—particularly in a country where regions, divisions, states, counties, rurality/urbanicity, and culturally shaped areas such as the "Bible Belt" have different effects on citizens' health and well-being (e.g., Barton 2010; Cossman et al. 2007; Farina et al. 2021; Garreau 1981; Hasenbush et al. 2014; James, Wolf, and Cossman 2020; Meyer et al. 2019; Montez 2020; Murray et al. 2006; Patterson et al. 2004; Woodard 2011). This dissertation is a first step in exploring health-related outcomes by sexual orientation across the US as a whole, and while non-significant findings between some minority groups and their majorities counterparts is good news, it is not a confirmation that prejudice or discrimination is not affecting the health of sexual minority group members.

Research has demonstrated relationships between sexual minority status and adverse mental health outcomes. Negative cultural attitudes and social/legal factors have been found to contribute to these issues (Barton 2010; Hatzenbuehler 2010). The stigma influencing such policies and the minority stress generated from them adversely affect the health of sexual minorities (Hatzenbuehler and Pachankis 2016; Meyer 2003). Positive public policy such as legalizing same-sex marriage can have beneficial effects for sexual minority health (Buffie 2011). In order for sexual orientation to be a strong negative driver of health, it needs to be in an 
area where it is stigmatized or perceived to be stigmatized. While reducing sexual orientation stigmatization is absolutely what we want to see and is occurring nationally over time, it opens the door to more demographically diverse people using a sexual minority identity and adds further complexities to the stigma-health relationship (Gates 2013). Thus, we can still consider stigma a fundamental cause of health disparities (Hatzenbuehler et al. 2013). Future research needs to consider the specific context in which sexual minorities are living, the environment in which they grew up, and continue to use an intersectional perspective monitoring the effects of other intertwined stigmatized statuses.

Interestingly, other social conditions typically considered fundamental causes of disease, such as household income measured as percent of federal poverty level and educational attainment, were strongly associated with higher odds of good SRH but were less so with the other two health-related outcomes in this study. In the full sample, both social conditions showed a strong gradient of increasingly higher odds of good SRH as income and education increased compared to their reference groups. Household income was only significantly associated with lower relative risk of obesity for those at $300 \%$ or greater of the poverty level compared to those at $0-99 \%$ of the poverty level. Neither level (100-299\% and $>300 \%)$ differed from the reference category regarding overweight. Regarding educational attainment, only those with a college degree or more had lower relative risk of overweight or obesity compared to their less than high school counterparts. Only those with household incomes at $300 \%$ or greater than the federal poverty level had higher odds of access to a USOC. Those with some college or a college degree or more had higher odds of access to a USOC while those with a high school diploma or GED had no significant difference from those with less than a high school degree. As such, perhaps different variables regarding body fat or health care access should have been assessed.

In some analyses, a sexual minority status was significantly related to overweight, obesity, or access to a USOC in the unadjusted models, but once adjusted, the significance disappeared. However, despite adjustments, sexual orientation continued to be a significant predictor of poor SRH throughout models on the full sample. Significance only disappeared regarding SRH for two statuses (i.e., bisexual, equally attracted to males and females) among those in the older age group sample and for one status (i.e., only attracted to the same sex) in the female sample once fuller models were considered. 


\section{LIMITATIONS}

While these chapters provide multiple new views into different health factors by sexual orientation, they are not without their limitations. These three chapters give a detailed overview of the relationships between three components of sexual orientation and self-rated health (SRH), body mass index (BMI), and access to a usual source of health care while accounting for sociodemographic/economic factors, but they do not account for contextual factors (e.g., cultural, social, political, or physical environments) due to the restricted nature of the publicly available data. Health literature shows that contextual factors such as location (e.g., Woodell 2018), local laws (e.g., Meyer et al. 2019), religious environments (e.g., Hatzenbuehler, Pachankis, and Wolff 2012), and partnership status (e.g., Reczek et al. 2017) are also associated with health outcomes by sexual orientation and in general. Contextual data would also help illuminate where stigma around sexual orientation was particularly salient and potentially more likely to affect healthrelated outcomes. Lack of publicly available environmental data makes it difficult to explore how people become "at risk of risks" as discussed by Link and Phelan (1995) who advocate for contextualizing individually-based risk factors. Although examining national patterns in social statuses and health outcomes is informative, it would be even more insightful to investigate where these relationships are heightened or lessened by other contextual factors to concentrate efforts and resources to effect positive change.

Similarly, respondents from the years before and after same-sex marriage legalization in 2015 (Anon 2015) were combined together for analyses. Marriage is beneficial to health by providing financial and social support (Buffie 2011; Umberson and Montez 2010). The combined sample over nine years is also subject to other historical/contextual differences that are unaccounted for in these analyses (Gates 2013); however, I control for survey cycle to attempt to circumvent this limitation. Due to the cross-sectional nature of the data, these chapters also cannot explore changes in sexual orientation (e.g., Mock and Eibach 2012) or health across the life course. Sexual minority youth are at greater risk of exposure to adverse or stressful childhood experiences which can negatively affect later life outcomes (Felitti et al. 1998; Schneeberger et al. 2014; Soleimanpour, Geierstanger, and Brindis 2017). More people are reporting a sexual minority identity today as well (Jones 2021), most likely indicating greater acceptance of doing so rather than simply more sexual minority people existing (Gates 2013). This finding and another poll on growing attitudes supporting same-sex marriage lend support to 
a decrease in stigma nationally around sexual orientation (McCarthy 2018). Consideration of historical context and life course changes would provide a better picture of the mechanisms driving health outcomes.

Another limitation of this data is that the NSFG splits its samples into male and female, completely ignoring the gender and sex spectrums. While convenient for situating these analyses in the literature that mainly focuses on two genders and/or sexes, this methodological shortcoming erases the experiences of those who live outside the gender and sex binary (Matsuno 2019). Likewise, for ease of comparison across much of the literature, these chapters do not account for those who responded "refused," "don't know," "not sure," or "something else." While some researchers have included them (e.g., Boehmer et al. 2007; Fish, Hughes, and Russell 2018), it was beyond the scope of analyses at this time for this study.

The results regarding respondents' BMI are to also be interpreted with caution. BMI is a measure that has been questioned for its accuracy in assessing health (Nuttall 2015), though underweight and obesity have been found to be associated with higher levels of mortality (Flegal et al. 2005) and obesity in particular puts adults at an increased risk of a variety of other health problems (Office of the Surgeon General (US) 2010). The NSFG did not consistently ask about diet or exercise patterns which could have provided more insight into respondents' BMI categorization and health. The small size of the NSFG sample respondents who reported an underweight BMI also prohibited analyses further exploring this weight category.

Finally, these chapters only consider one aspect of health care access. I do not investigate measures such as frequency of specific types of health care use (e.g., screenings and tests) as other studies have (e.g., Conron et al. 2010; Fredriksen-Goldsen, Kim, et al. 2013; Strutz et al. 2015). Quality of available care was also not assessed which is important when considering sexual minority health; research has found health care establishments as places of discrimination among sexual minorities (Hafeez et al. 2017; Matsuno 2019). While limited, these current chapters do provide a descriptive blueprint for future research.

\section{FUTURE RESEARCH}

Acknowledging these chapters' limitations helps guide the path for future related research. The NSFG does have contextual data, but it is restricted and requires approved access to it. Obtaining this data would allow for a more nuanced exploration of the role environmental factors play in the sexual orientation-health relationship. Additionally, more health-related 
behaviors and outcomes such as those related to reproductive health or coping mechanisms (e.g., alcohol or substance use/abuse) are available in the NSFG and information on mental and physical health are available in other datasets such as the National Longitudinal Study of Adolescent to Adult Health (Add Health). Using Add Health would allow for a longitudinal examination of sexual orientation and health outcomes as well while accounting for contextual factors with their restricted-use data.

Future research can more deeply examine racial and ethnic differences of sexual orientation components on health-related factors (Worthen 2013) or how sexual orientation is related to health for different education attainment groups. Chen and Yang (2014) found support for a framework that showed racial discrimination as directly influencing self-rated health and as indirectly influencing it via health behaviors and neighborhood social capital. The same framework could be applied to studies on sexual minority discrimination and health outcomes. I can also explore how health-related outcomes may vary by inconsistent sexual orientation components (e.g., a female attracted to both males and females who only has sex with males and identifies as heterosexual). Experienced discrimination and prejudice may then vary if others do not perceive them as sexual minorities. Data on perceived discrimination by social status (e.g., race/ethnicity, sexual orientation, and sex or gender) would also be helpful in illuminating what types of stressors are driving health-related outcomes perhaps more strongly than others.

Age, period, and cohort analyses would provide greater understanding of the health of sexual minorities. My dissertation looked at age differences in health by sexual orientation component, but as noted in the limitations, it does not consider context or period effects on health nor does it fully consider the cohort effects of growing up in different eras. The Generations Study was created to explore different birth cohorts who "came of age during distinctly different historical contexts" under the guide of minority stress theory which maintains that the legal and social environments in which different children grew up would convey different amounts of stigma and stress for sexual minorities (Krueger et al. 2020:4; Meyer 2003). Due to the quickly changing intensity of stigma surrounding sexual orientation in the United States over the past few decades (Twenge, Sherman, and Wells 2016), make this a research avenue ripe to pursue while also considering how stigma can be a fundamental cause of health disparities as it evolves dynamically across age, periods, and cohorts (Hatzenbuehler and Pachankis 2016; Hatzenbuehler et al. 2013). Twenge and colleagues (2016) have already 
observed marked increases in same-sex sexual behaviors, which they find mainly attributable to time period, and Jones (2021) illustrates the increase in sexual minority identity over time and the higher prevalence in younger cohorts. Further investigation of age-period-cohort effects on health disparities by sexual orientation, particularly by smaller contextual regions and in the US overall would provide greater insight into drivers of such inequities as well as ideas of what to target to reduce them.

Several datasets currently exist asking about sexual orientation that would be advantageous to use to contribute to the sexual minority health literature: Behavioral Risk Factor Surveillance System (BRFSS); General Social Survey (GSS); National Epidemiologic Survey on Alcohol and Related Conditions (NESARC); National Longitudinal Study of Adolescent to Adult Health (Add Health); National Survey of Midlife Development in the United States (MIDUS); The Generations Study. As more data becomes available (e.g., particularly longitudinal data) over time, I can continue to address the limitations of these chapters and contribute to this growing field of sexual minority health.

\section{CONCLUSION}

National exploration into sexual minority health disparities is still in its infancy. Lack of data and inconsistencies in sexual orientation measurement have limited the ability to accurately assess health-related outcomes; however, available research has generally found poorer health behaviors, coping mechanisms, health care utilization/experiences, mental health, and physical health among sexual orientation minorities compared to their heterosexual counterparts. Stigma and minority stress are often cited as the root causes of these health inequities. This dissertation operates under the assumption of equal stigma and stress across the nation that differs solely by social statuses due to its lack of including restricted-access contextual data. Some areas of the United States are steeped in greater volumes of stigma and consequential anti-LGBTQ+ policies and cultural environments that adversely affect sexual minority health. Without this data, it is impossible to tell where health disparities are more or less prevalent. Despite this limitation, national patterns of poorer self-rated health among those who have had same-sex sexual experiences and of mainly younger and female bisexuals have emerged.

Significantly lower odds of having good, very good, or excellent self-rated health is particularly troubling due to this subjective assessment's close connection to morbidities and mortality. Finding this result at the national-level in a sample combined over a nine-year period 
including the legalization of same-sex marriage and growing acceptance of sexual minorities is disquieting. Bisexuals in the overall sample, but more specifically among those 25-34 years-old, were also at higher odds of reporting obesity than their heterosexual counterparts. Those who identify as bisexual are at greater risk of prejudice and discrimination from both heterosexuals, gay men, and lesbians. This excess stress and lack of sexual minority community support may be driving their sustained poor health outcomes. Future research should pay particular attention to this group's experiences of stigma and stress, especially as use of this identity continues to increase, as well as keep in mind other intersectional identities such as sex and age.

On the other hand, not all findings from these analyses appear negative. Most sexual minority males across all component statuses had significantly higher odds of having a usual source of healthcare as did those in the younger age group who identify as gay/lesbian and those mostly attracted to the same sex. These results may be indications of taking more preventive care measures among these groups. Additionally, males who identify as gay have lower odds of being overweight or obese compared to their heterosexual counterparts. While this finding might seem like a sign of good physical health, it may be a reflection of poorer mental health among this population as literature reveals more body shape dissatisfaction among sexual minority men. Future studies should consider mental and physical health together among sexual minorities.

Research with contextual measures of stigma and respondents' perceived discrimination and stress can more firmly unite fundamental cause and minority stress theories beyond only operating under their frameworks. This dissertation has revealed significant country-level relationships between sexual orientation components and self-rated health, body mass index, and access to a usual source of health care and examined how sex and age intersect with these associations, but it is only a foundation for more nuanced explorations into sexual minority health. As more data on sexual minorities becomes available scholars can investigate and compare health-related trends across the United States and work to eliminate health disparities. 


\section{REFERENCES}

Almeida, Joanna, Renee M. Johnson, Heather L. Corliss, Beth E. Molnar, and Deborah Azrael. 2009. "Emotional Distress Among LGBT Youth: The Influence of Perceived Discrimination Based on Sexual Orientation." Journal of Youth \& Adolescence 38(7):1001-14. doi: 10.1007/s10964-0099397-9.

Aneshensel, Carol S. 1992. "Social Stress: Theory and Research." Annual Review of Sociology 18:15-38.

Anon. 2015. Obergefell v. Hodges.

Anon. 2020. "Generations.” Generations. Retrieved March 27, 2020 (http://www.generations-study.com).

Austin, S. Bryn, Lauren A. Nelson, Michelle A. Birkett, Jerel P. Calzo, and Bethany Everett. 2013. "Eating Disorder Symptoms and Obesity at the Intersections of Gender, Ethnicity, and Sexual Orientation in US High School Students." American Journal Of Public Health 103(2):e16-22. doi: 10.2105/AJPH.2012.301150.

Austin, S. Bryn, Najat J. Ziyadeh, Heather L. Corliss, Jess Haines, Helaine R. Rockett, David Wypij, and Alison E. Field. 2009. "Sexual Orientation Disparities in Weight Status in Adolescence: Findings From a Prospective Study." Obesity 17(9):1776-82. doi: 10.1038/oby.2009.72.

Badgett, M. V. Lee, Laura E. Durso, and Alyssa Schneebaum. 2013. New Patterns of Poverty in the Lesbian, Gay, and Bisexual Community. Los Angeles, CA: The Williams Institute.

Badgett, M. V. Lee, Brad Sears, Holning Lau, and Deborah Ho. 2009. "Bias in the Workplace: Consistent Evidence of Sexual Orientation and Gender Identity Discrimination 1998-2008 Symposium: The Evolution of Academic Discourse on Sexual Orientation and the Law: A Festschrift in Honor of Jeffrey Sherman." Chicago-Kent Law Review 84(2):559-96.

Barefoot, K. Nikki, Jacob C. Warren, and K. Bryant Smalley. 2015. "An Examination of Past and Current Influences of Rurality on Lesbians' Overweight/Obesity Risks.” LGBT Health 2(2):154-61. doi: 10.1089/lgbt.2014.0112.

Barnes, David M., Mark L. Hatzenbuehler, Ava D. Hamilton, and Katherine M. Keyes. 2014. "Sexual Orientation Disparities in Mental Health: The Moderating Role of Educational Attainment." Social Psychiatry and Psychiatric Epidemiology 49(9):1447-54. doi: 10.1007/s00127-014-08495.

Barton, Bernadette. 2010. “'Abomination'-Life as a Bible Belt Gay.” Journal of Homosexuality 57(4):465-84. doi: 10.1080/00918361003608558.

Bauer, Greta R., and Jennifer A. Jairam. 2008. "Are Lesbians Really Women Who Have Sex with Women (WSW)? Methodological Concerns in Measuring Sexual Orientation in Health Research." Women \& Health 48(4):383-408. doi: 10.1080/03630240802575120.

Bauer, Greta R., Jennifer A. Jairam, and Shamara M. Baidoobonso. 2010. "Sexual Health, Risk Behaviors, and Substance Use in Heterosexual-Identified Women With Female Sex Partners: 2002 US National Survey of Family Growth." Sexually Transmitted Diseases 37(9):531-37. doi: 10.1097/OLQ.0b013e3181d785f4. 
Bennett, K. 1992. "Feminist Bisexuality.” Pp. 205-31 in Close to Home: Bisexuality and Feminism, edited by E. R. Weise. Seattle, WA: The Seal Press.

Bernard, Paul, Rana Charafeddine, Katherine L. Frohlich, Mark Daniel, Yan Kestens, and Louise Potvin. 2007. "Health Inequalities and Place: A Theoretical Conception of Neighbourhood." Social Science \& Medicine 65(9):1839-52. doi: 10.1016/j.socscimed.2007.05.037.

Bird, Jason D. P., Lisa Kuhns, and Robert Garofalo. 2012. "The Impact of Role Models on Health Outcomes for Lesbian, Gay, Bisexual, and Transgender Youth.” Journal of Adolescent Health 50(4):353-57. doi: 10.1016/j.jadohealth.2011.08.006.

Blosnich, John, and Robert Bossarte. 2012. "Drivers of Disparity: Differences in Socially Based Risk Factors of Self-Injurious and Suicidal Behaviors Among Sexual Minority College Students." Journal of American College Health 60(2):141-49. doi: 10.1080/07448481.2011.623332.

Blosnich, John R., Grant W. Farmer, Joseph G. L. Lee, Vincent M. B. Silenzio, and Deborah J. Bowen. 2014. "Health Inequalities Among Sexual Minority Adults: Evidence from Ten U.S. States, 2010." American Journal of Preventive Medicine 46(4):337-49. doi: 10.1016/j.amepre.2013.11.010.

Boehmer, Ulrike, and Deborah J. Bowen. 2009. "Examining Factors Linked to Overweight and Obesity in Women of Different Sexual Orientations.” Preventive Medicine 48(4):357-61. doi: 10.1016/j.ypmed.2009.02.003.

Boehmer, Ulrike, Deborah J. Bowen, and Greta R. Bauer. 2007. "Overweight and Obesity in SexualMinority Women: Evidence From Population-Based Data.” American Journal of Public Health 97(6):1134-40. doi: 10.2105/AJPH.2006.088419.

Bontempo, Daniel E., and Anthony R. D’Augelli. 2002. "Effects of At-School Victimization and Sexual Orientation on Lesbian, Gay, or Bisexual Youths' Health Risk Behavior." Journal of Adolescent Health 30(5):364-74. doi: 10.1016/S1054-139X(01)00415-3.

Bostwick, Wendy B., Tonda L. Hughes, and Bethany Everett. 2015. "Health Behavior, Status, and Outcomes Among a Community-Based Sample of Lesbian and Bisexual Women." LGBT Health 2(2):121-26. doi: 10.1089/lgbt.2014.0074.

Bowen, Deborah J., Kimberly F. Balsam, and Samantha R. Ender. 2008. "A Review of Obesity Issues in Sexual Minority Women.” Obesity 16(2):221-28. doi: 10.1038/oby.2007.34.

Bränström, Richard, Mark L. Hatzenbuehler, John E. Pachankis, and Bruce G. Link. 2016. "Sexual Orientation Disparities in Preventable Disease: A Fundamental Cause Perspective.” American Journal of Public Health 106(6):1109-15. doi: 10.2105/AJPH.2016.303051.

Brennan, David J., Kenta Asakura, Clemon George, Peter A. Newman, Sulaimon Giwa, Trevor A. Hart, Rusty Souleymanov, and Gerardo Betancourt. 2013. “Never Reflected Anywhere': Body Image Among Ethnoracialized Gay and Bisexual Men." Body Image 10(3):389-98. doi: 10.1016/j.bodyim.2013.03.006.

Brennan-Ing, Mark, Liz Seidel, Britta Larson, and Stephen E. Karpiak. 2014. "Social Care Networks and Older LGBT Adults: Challenges for the Future.” Journal of Homosexuality 61(1):21-52. doi: 10.1080/00918369.2013.835235. 
Brewster, Karin L., and Kathryn Harker Tillman. 2011. "Sexual Orientation and Substance Use Among Adolescents and Young Adults." American Journal of Public Health 102(6):1168-76. doi: 10.2105/AJPH.2011.300261.

Buffie, William C. 2011. "Public Health Implications of Same-Sex Marriage.” American Journal of Public Health 101(6):986-90. doi: 10.2105/AJPH.2010.300112.

Callis, April Scarlette. 2013. "The Black Sheep of the Pink Flock: Labels, Stigma, and Bisexual Identity." Journal of Bisexuality 13:82-105. doi: 10.1080/15299716.2013.755730.

CDC. 2020. "All About Adult BMI." Centers for Disease Control and Prevention. Retrieved December 29, 2020 (https://www.cdc.gov/healthyweight/assessing/bmi/adult_bmi/index.html).

Centers for Disease Control and Prevention. 2019. "Calculating BMI Using the English System - BMI for Age Training Course.” Retrieved December 29, 2020

(http://www.cdc.gov/nccdphp/dnpao/growthcharts/training/bmiage/page5_2.html).

Centers for Disease Control and Prevention. 2020. "Defining Adult Overweight and Obesity." Retrieved November 5, 2020 (https://www.cdc.gov/obesity/adult/defining.html).

Chaudhry, Aeysha B., and Sari L. Reisner. 2019. "Disparities by Sexual Orientation Persist for Major Depressive Episode and Substance Abuse or Dependence: Findings from a National Probability Study of Adults in the United States." LGBT Health 6(5):261-66. doi: 10.1089/lgbt.2018.0207.

Chen, Danhong, and Tse-Chuan Yang. 2014. "The Pathways from Perceived Discrimination to Self-Rated Health: An Investigation of the Roles of Distrust, Social Capital, and Health Behaviors." Social Science \& Medicine 104:64-73. doi: 10.1016/j.socscimed.2013.12.021.

Cochran, Susan D., and Vickie M. Mays. 2014. "Mortality Risks Among Persons Reporting Same-Sex Sexual Partners: Evidence From the 2008 General Social Survey-National Death Index Data Set." American Journal of Public Health 105(2):358-64. doi: 10.2105/AJPH.2014.301974.

Cohen, Steven A., Sarah K. Cook, Lauren Kelley, Julia D. Foutz, and Trisha A. Sando. 2017. “A Closer Look at Rural-Urban Health Disparities: Associations Between Obesity and Rurality Vary by Geospatial and Sociodemographic Factors." The Journal of Rural Health 33(2):167-79. doi: 10.1111/jrh.12207.

Coker, Tumaini R., S. Bryn Austin, and Mark A. Schuster. 2010. "The Health and Health Care of Lesbian, Gay, and Bisexual Adolescents." Annual Review of Public Health 31(1):457-77. doi: 10.1146/annurev.publhealth.012809.103636.

Conron, Keith J., Matthew J. Mimiaga, and Stewart J. Landers. 2008. “A Health Profile of Massachusetts Adults by Sexual Orientation Identity: Results from the 2001-2006 Behavioral Risk Factor Surveillance System Surveys." 1-30.

Conron, Kerith J., Matthew J. Mimiaga, and Stewart J. Landers. 2010. “A Population-Based Study of Sexual Orientation Identity and Gender Differences in Adult Health." American Journal of Public Health 100(10):1953-60. doi: 10.2105/AJPH.2009.174169. 
Cossman, Jeralynn Sittig, Ronald E. Cossman, Wesley L. James, Carol R. Campbell, Troy C. Blanchard, and Arthur G. Cosby. 2007. "Persistent Clusters of Mortality in the United States." American Journal of Public Health 97(12):2148-50. doi: 10.2105/AJPH.2006.093112.

Cummins, Steven, Sarah Curtis, Ana V. Diez-Roux, and Sally Macintyre. 2007. "Understanding and Representing 'Place' in Health Research: A Relational Approach." Social Science \& Medicine 65(9):1825-38. doi: 10.1016/j.socscimed.2007.05.036.

Daniels, Stephen R., Donna K. Arnett, Robert H. Eckel, Samuel S. Gidding, Laura L. Hayman, Shiriki Kumanyika, Thomas N. Robinson, Barbara J. Scott, Sachiko St Jeor, and Christine L. Williams. 2005. "Overweight in Children and Adolescents Pathophysiology, Consequences, Prevention, and Treatment." Circulation 111(15):1999-2012. doi: 10.1161/01.CIR.0000161369.71722.10.

Deputy, Nicholas P., and Ulrike Boehmer. 2010. "Determinants of Body Weight Among Men of Different Sexual Orientation." Preventive Medicine 51(2):129-31. doi: 10.1016/j.ypmed.2010.05.010.

Diamant, Allison L., Cheryl Wold, Karen Spritzer, and Lillian Gelberg. 2000. "Health Behaviors, Health Status, and Access to and Use of Health Care: A Population-Based Study of Lesbian, Bisexual, and Heterosexual Women." Archives of Family Medicine 9(10):1043-51.

Dilley, Julia A., Katrina Wynkoop Simmons, Michael J. Boysun, Barbara A. Pizacani, and Mike J. Stark. 2010. "Demonstrating the Importance and Feasibility of Including Sexual Orientation in Public Health Surveys: Health Disparities in the Pacific Northwest." American Journal of Public Health 100(3):460-67. doi: 10.2105/AJPH.2007.130336.

DuRant, Robert H., Daniel P. Krowchuk, and Sara H. Sinal. 1998. "Victimization, Use of Violence, and Drug Use at School among Male Adolescents Who Engage in Same-Sex Sexual Behavior." The Journal of Pediatrics 133(1):113-18. doi: 10.1016/S0022-3476(98)70189-1.

Durso, Laura E., and Gary J. Gates. 2013. "Best Practices: Collecting and Analyzing Data on Sexual Minorities.” Pp. 21-42 in International Handbook on the Demography of Sexuality, International Handbooks of Population, edited by A. K. Baumle. Springer Netherlands.

Eberhardt, MS, DD Ingram, and DM Makuc. 2001. Urban and Rural Health Chartbook. Health, United States, 2001. Hyattsville, MD: National Center for Health Statistics.

Elia, John P. 1993. "Homophobia in the High School: A Problem in Need of a Resolution." The High School Journal 77(1/2):177-85.

Everett, Bethany. 2015. "Sexual Orientation Identity Change and Depressive Symptoms A Longitudinal Analysis." Journal of Health and Social Behavior 56(1):37-58. doi: 10.1177/0022146514568349.

Farina, Mateo P., Anna Zajacova, Jennifer Karas Montez, and Mark D. Hayward. 2021. "US State Disparities in Life Expectancy, Disability-Free Life Expectancy, and Disabled Life Expectancy Among Adults Aged 25 to 89 Years." American Journal of Public Health e1-10. doi: 10.2105/AJPH.2020.306064.

Farmer, Melissa M., and Kenneth F. Ferraro. 1997. "Distress and Perceived Health: Mechanisms of Health Decline.” Journal of Health and Social Behavior 38(3):298-311. doi: 10.2307/2955372. 
Faulkner, A. H., and K. Cranston. 1998. "Correlates of Same-Sex Sexual Behavior in a Random Sample of Massachusetts High School Students." American Journal of Public Health 88(2):262-66. doi: 10.2105/AJPH.88.2.262.

Felitti, Vincent J., Robert F. Anda, Dale Nordenberg, David F. Williamson, Alison M. Spitz, Valerie Edwards, Mary P. Koss, and James S. Marks. 1998. "Relationship of Childhood Abuse and Household Dysfunction to Many of the Leading Causes of Death in Adults: The Adverse Childhood Experiences (ACE) Study." American Journal of Preventive Medicine 14(4):245-58. doi: 10.1016/S0749-3797(98)00017-8.

Ferraro, Kenneth F., Melissa M. Farmer, and John A. Wybraniec. 1997. "Health Trajectories: Long-Term Dynamics Among Black and White Adults." Journal of Health and Social Behavior 38(1):38-54. doi: $10.2307 / 2955360$.

Ferraro, Kenneth F., and Ya-ping Su. 2000. "Physician-Evaluated and Self-Reported Morbidity for Predicting Disability." American Journal of Public Health 90(1):103-8.

Fish, Jessica N. 2019. "Sexual Orientation-Related Disparities in High-Intensity Binge Drinking: Findings from a Nationally Representative Sample.” LGBT Health 6(5):242-49. doi: 10.1089/lgbt.2018.0244.

Fish, Jessica N., Tonda L. Hughes, and Stephen T. Russell. 2018. "Sexual Identity Differences in HighIntensity Binge Drinking: Findings from a US National Sample.” Addiction 113(4):749-58. doi: 10.1111/add.14041.

Fish, Jessica N., and Kay Pasley. 2015. "Sexual (Minority) Trajectories, Mental Health, and Alcohol Use: A Longitudinal Study of Youth as They Transition to Adulthood." Journal of Youth and Adolescence 44(8):1508-27. doi: http://dx.doi.org.www.libproxy.wvu.edu/10.1007/s10964-0150280-6.

Flegal, Katherine M., Barry I. Graubard, David F. Williamson, and Mitchell H. Gail. 2005. "Excess Deaths Associated With Underweight, Overweight, and Obesity." JAMA 293(15):1861-67. doi: 10.1001/jama.293.15.1861.

Flenar, Delphia J., Carolyn M. Tucker, and Jaime L. Williams. 2017. "Sexual Minority Stress, Coping, and Physical Health Indicators." Journal of Clinical Psychology in Medical Settings 24(3):22333. doi: 10.1007/s10880-017-9504-0.

Fredriksen-Goldsen, Karen I., Charles A. Emlet, Hyun-Jun Kim, Anna Muraco, Elena A. Erosheva, Jayn Goldsen, and Charles P. Hoy-Ellis. 2013. "The Physical and Mental Health of Lesbian, Gay Male, and Bisexual (LGB) Older Adults: The Role of Key Health Indicators and Risk and Protective Factors.” The Gerontologist 53(4):664-75. doi: 10.1093/geront/gns123.

Fredriksen-Goldsen, Karen I., Hyun-Jun Kim, Susan E. Barkan, Anna Muraco, and Charles P. Hoy-Ellis. 2013. "Health Disparities Among Lesbian, Gay, and Bisexual Older Adults: Results From a Population-Based Study." American Journal of Public Health 103(10):1802-9. doi: 10.2105/AJPH.2012.301110.

Frost, David M., Keren Lehavot, and Ilan H. Meyer. 2015. "Minority Stress and Physical Health Among Sexual Minority Individuals." Journal of Behavioral Medicine 38(1):1-8. doi: 10.1007/s10865013-9523-8. 
Frost, David M., Ilan H. Meyer, and Sharon Schwartz. 2016. "Social Support Networks among Diverse Sexual Minority Populations.” American Journal of Orthopsychiatry 86(1):91-102. doi: 10.1037/ort0000117.

Funders for Lesbian and Gay Issues. 2004. Aging In Equity: LGBT Elders in America.

Garofalo, Robert, R. Cameron Wolf, Shari Kessel, Judith Palfrey, and Robert H. DuRant. 1998. "The Association Between Health Risk Behaviors and Sexual Orientation Among a School-Based Sample of Adolescents." Pediatrics 101(5):895-902. doi: 10.1542/peds.101.5.895.

Garreau, Joel. 1981. The Nine Nations of North America. Boston: Houghton Mifflin.

Gates, Gary J. 2011. How Many People Are Lesbian, Gay, Bisexual, and Transgender? The Williams Institute.

Gates, Gary J. 2013. "Demographics and LGBT Health.” Journal of Health and Social Behavior 54(1):72-74. doi: 10.1177/0022146512474429.

Gates, Gary J., and Frank Newport. 2012. Special Report: 3.4\% of U.S. Adults Identify as LGBT. Gallup.

Gonzales, Gilbert, Julia Przedworski, and Carrie Henning-Smith. 2016. "Comparison of Health and Health Risk Factors Between Lesbian, Gay, and Bisexual Adults and Heterosexual Adults in the United States: Results From the National Health Interview Survey.” JAMA Internal Medicine 176(9):1344-51. doi: 10.1001/jamainternmed.2016.3432.

Gorman, Bridget K., Justin T. Denney, Hilary Dowdy, and Rose Anne Medeiros. 2015. "A New Piece of the Puzzle: Sexual Orientation, Gender, and Physical Health Status." Demography 52(4):135782. doi: 10.1007/s13524-015-0406-1.

Grant, Jaime M. 2010. Outing Age 2010: Public Policy Issues Affecting Lesbian, Gay, Bisexual, and Transgender Elders. Washington, DC: National Gay and Lesbian Task Force Policy Institute.

Hafeez, Hudaisa, Muhammad Zeshan, Muhammad A. Tahir, Nusrat Jahan, and Sadiq Naveed. 2017. "Health Care Disparities Among Lesbian, Gay, Bisexual, and Transgender Youth: A Literature Review." Cureus 9(4):e1184. doi: 10.7759/cureus.1184.

Hasenbush, Amira, Andrew R. Flores, Angeliki Kastanis, Brad Sears, and Gary J. Gates. 2014. The LGBT Divide: A Data Portrait of LGBT People in the Midwestern, Mountain \& Southern States. The Williams Institute.

Hatzenbuehler, Mark L. 2009. "How Does Sexual Minority Stigma 'Get Under the Skin'? A Psychological Mediation Framework.” Psychological Bulletin 135(5):707-30. doi: 10.1037/a0016441.

Hatzenbuehler, Mark L. 2010. "Social Factors as Determinants of Mental Health Disparities in LGB Populations: Implications for Public Policy.” Journal of Social Issues 4(1):31-62. doi: 10.1111/j.1751-2409.2010.01017.x@10.1111/(ISSN)15404560.TOWARDANUNDERSTANDINGOFTHEORLANDOMASSACRE.

Hatzenbuehler, Mark L., and John E. Pachankis. 2016. "Stigma and Minority Stress as Social Determinants of Health Among Lesbian, Gay, Bisexual, and Transgender Youth: Research 
Evidence and Clinical Implications.” Pediatric Clinics 63(6):985-97. doi:

10.1016/j.pcl.2016.07.003.

Hatzenbuehler, Mark L., John E. Pachankis, and Joshua Wolff. 2012. "Religious Climate and Health Risk Behaviors in Sexual Minority Youths: A Population-Based Study." American Journal of Public Health 102(4):657-63. doi: 10.2105/AJPH.2011.300517.

Hatzenbuehler, Mark L., Jo C. Phelan, and Bruce G. Link. 2013. "Stigma as a Fundamental Cause of Population Health Inequalities." American Journal of Public Health 103(5):813-21. doi: 10.2105/AJPH.2012.301069.

Healthy People 2020. 2020. "Lesbian, Gay, Bisexual, and Transgender Health.” Retrieved February 22, 2021 (https://www.healthypeople.gov/2020/topics-objectives/topic/lesbian-gay-bisexual-andtransgender-health).

Herrick, Shannon S. C., and Lindsay R. Duncan. 2018. "A Systematic Scoping Review of Engagement in Physical Activity Among LGBTQ+ Adults." Journal of Physical Activity \& Health 15(3):226-32.

Hottes, Travis Salway, Laura Bogaert, Anne E. Rhodes, David J. Brennan, and Dionne Gesink. 2016. "Lifetime Prevalence of Suicide Attempts Among Sexual Minority Adults by Study Sampling Strategies: A Systematic Review and Meta-Analysis." American Journal of Public Health 106(5):e1-12. doi: 10.2105/AJPH.2016.303088.

Hsieh, Ning, and Matt Ruther. 2016. "Sexual Minority Health and Health Risk Factors: Intersection Effects of Gender, Race, and Sexual Identity." American Journal of Preventive Medicine 50(6):746-55. doi: 10.1016/j.amepre.2015.11.016.

$\mathrm{Hu}$, Yueqin, Yishan Xu, and Samantha L. Tornello. 2015. "Stability of Self-Reported Same-Sex and Both-Sex Attraction from Adolescence to Young Adulthood." Archives of Sexual Behavior 45(3):651-59. doi: 10.1007/s10508-015-0541-1.

Idler, Ellen L., and Stanislav V. Kasl. 1995. "Self-Ratings of Health: Do They Also Predict Change in Functional Ability?" The Journals of Gerontology: Series B 50B(6):S344-53. doi: 10.1093/geronb/50B.6.S344.

Igartua, Karine, Brett D. Thombs, Giovani Burgos, and Richard Montoro. 2009. "Concordance and Discrepancy in Sexual Identity, Attraction, and Behavior Among Adolescents." Journal of Adolescent Health 45(6):602-8. doi: 10.1016/j.jadohealth.2009.03.019.

Institute of Medicine. 2011. The Health of Lesbian, Gay, Bisexual, and Transgender People: Building a Foundation for Better Understanding. Washington, DC: The National Academies Press.

James, Wesley, Julia Kay Wolf, and Jeralynn Cossman. 2020. “Characteristics of Persistently Unhealthy Counties: A Comparison of White and Black Mortality Over Time.” The Social Science Journal 57(1):115-19. doi: 10.1016/j.soscij.2019.01.005.

Jones, Jeffrey M. 2021. "LGBT Identification Rises to 5.6\% in Latest U.S. Estimate." Gallup.Com. Retrieved February 25, 2021 (https://news.gallup.com/poll/329708/lgbt-identification-rises-latestestimate.aspx). 
Jylhä, M. 2009. "What Is Self-Rated Health and Why Does It Predict Mortality? Towards a Unified Conceptual Model.” Social Science \& Medicine 69:307-16.

Katz-Wise, Sabra L., Emily A. Blood, Carly E. Milliren, Jerel P. Calzo, Tracy K. Richmond, Holly C. Gooding, and S. Bryn Austin. 2014. "Sexual Orientation Disparities in BMI among US Adolescents and Young Adults in Three Race/Ethnicity Groups." Journal of Obesity 2014:e537242. doi: 10.1155/2014/537242.

Kelleher, C. 2009. "Minority Stress and Health: Implications for Lesbian, Gay, Bisexual, Transgender, and Questioning (LGBTQ) Young People.” Counselling Psychology Quarterly 22(4):373-79. doi: $10.1080 / 09515070903334995$.

Kinsky, Suzanne M. 2015. "Exploring Lesbian Health Disparities: Social and Structural Predictors of Adiposity and the Metabolic Syndrome." Dissertation, University of Pittsburgh, Pittsburgh, PA.

Kosciw, Joseph G., Caitlin M. Clark, Nhan L. Truong, and Adrian D. Zongrone. 2020. The 2019 National School Climate Survey: The Experiences of Lesbian, Gay, Bisexual, Transgender, and Queer Youth in Our Nation's Schools. New York: GLSEN.

Krueger, Evan A., Andy Lin, Krystal R. Kittle, and Ilan H. Meyer. 2020. Generations: A Study of the Life and Health of LGB People in a Changing Society: Methodology and Technical Notes Gallup Quantitative Survey.

Lee, J. G. L., G. K. Griffin, and C. L. Melvin. 2009. "Tobacco Use Among Sexual Minorities in the USA, 1987 to May 2007: A Systematic Review." Tobacco Control 18(4):275-82. doi:

$10.1136 /$ tc.2008.028241.

Lick, David J., Laura E. Durso, and Kerri L. Johnson. 2013. "Minority Stress and Physical Health Among Sexual Minorities." Perspectives on Psychological Science 8(5):521-48. doi: $10.1177 / 1745691613497965$.

Link, Bruce G., and Jo C. Phelan. 1995. "Social Conditions as Fundamental Causes of Disease." Journal of Health and Social Behavior 35(Extra Issue: Forty Years of Medical Sociology: The State of the Art and Directions for the Future):80-94.

Mahaffy, Kimberly A. 1996. "Cognitive Dissonance and Its Resolution: A Study of Lesbian Christians." Journal for the Scientific Study of Religion 35(4):392-402. doi: 10.2307/1386414.

Manor, Orly, Sharon Matthews, and Chris Power. 2000. "Dichotomous or Categorical Response? Analysing Self-Rated Health and Lifetime Social Class.” International Journal of Epidemiology 29(1):149-57. doi: 10.1093/ije/29.1.149.

Marshal, Michael P., Laura J. Dietz, Mark S. Friedman, Ron Stall, Helen A. Smith, James McGinley, Brian C. Thoma, Pamela J. Murray, Anthony R. D'Augelli, and David A. Brent. 2011. "Suicidality and Depression Disparities Between Sexual Minority and Heterosexual Youth: A Meta-Analytic Review." Journal of Adolescent Health 49(2):115-23. doi: 10.1016/j.jadohealth.2011.02.005.

Mason, Tyler B., and Robin J. Lewis. 2015. "Minority Stress, Depression, Relationship Quality, and Alcohol Use: Associations with Overweight and Obesity Among Partnered Young Adult Lesbians." LGBT Health 2(4):333-40. doi: 10.1089/lgbt.2014.0053. 
Matsuno, Emmie. 2019. "Nonbinary-Affirming Psychological Interventions." Cognitive and Behavioral Practice 26(4):617-28. doi: 10.1016/j.cbpra.2018.09.003.

Mayfield, Wayne. 2001. "The Development of an Internalized Homonegativity Inventory for Gay Men." Journal of Homosexuality 41(2):53-76. doi: 10.1300/J082v41n02_04.

Mays, Vickie M., and Susan D. Cochran. 2001. "Mental Health Correlates of Perceived Discrimination Among Lesbian, Gay, and Bisexual Adults in the United States." American Journal of Public Health 91(11):1869-76. doi: 10.2105/AJPH.91.11.1869.

McCarthy, Justin. 2018. "Two in Three Americans Support Same-Sex Marriage.” Gallup.Com. Retrieved February 25, 2021 (https://news.gallup.com/poll/234866/two-three-americans-support-sexmarriage.aspx).

McGregor, Bonnie A., Charles S. Carver, Michael H. Antoni, Sharlene Weiss, Susan E. Yount, and Gail Ironson. 2001. "Distress and Internalized Homophobia Among Lesbian Women Treated for Early Stage Breast Cancer." Psychology of Women Quarterly 25(1):1-9. doi: 10.1111/14716402.00001 .

Mereish, Ethan H. 2014. "The Weight of Discrimination: The Relationship Between Heterosexist Discrimination and Obesity Among Lesbian Women." Psychology of Sexual Orientation and Gender Diversity No Pagination Specified. doi: 10.1037/sgd0000056.

Meyer, Ilan H. 2003. "Prejudice, Social Stress, and Mental Health in Lesbian, Gay, and Bisexual Populations." Psychological Bulletin 129(5):674-97. doi: 10.1037/0033-2909.129.5.674.

Meyer, Ilan H., Feijun Luo, Bianca D. M. Wilson, and Deborah M. Stone. 2019. "Sexual Orientation Enumeration in State Antibullying Statutes in the United States: Associations with Bullying, Suicidal Ideation, and Suicide Attempts Among Youth." LGBT Health 6(1):9-14. doi: 10.1089/lgbt.2018.0194.

Mock, Steven E., and Richard P. Eibach. 2012. "Stability and Change in Sexual Orientation Identity Over a 10-Year Period in Adulthood." Archives of Sexual Behavior 41(3):641-48. doi: 10.1007/s10508-011-9761-1.

Molina, Yamile, Keren Lehavot, Blair Beadnell, and Jane Simoni. 2013. "Racial Disparities in Health Behaviors and Conditions Among Lesbian and Bisexual Women: The Role of Internalized Stigma." LGBT Health 1(2):131-39. doi: 10.1089/lgbt.2013.0007.

Montez, Jennifer Karas. 2020. "US State Polarization, Policymaking Power, and Population Health." The Milbank Quarterly 98(4):1033-52. doi: 10.1111/1468-0009.12482.

Mosher, William D., Anjani Chandra, and Jo Jones. 2005. "Sexual Behavior and Selected Health Measures: Men and Women 15-44 Years of Age, United States, 2002."

Mulick, Patrick S., and Lester W. Wright, Jr. 2002. "Examining the Existence of Biphobia in the Heterosexual and Homosexual Populations." Journal of Bisexuality 2(4):45-64. doi: 10.1300/J159v02n04_03.

Murray, Christopher J. L., Sandeep C. Kulkarni, Catherine Michaud, Niels Tomijima, Maria T. Bulzacchelli, Terrell J. Iandiorio, and Majid Ezzati. 2006. "Eight Americas: Investigating 
Mortality Disparities across Races, Counties, and Race-Counties in the United States." PLoS Med 3(9):e260. doi: 10.1371/journal.pmed.0030260.

National Center for Health Statistics. 2016. 2013-2015 NSFG USER'S GUIDE Appendix 6: Frequently Asked Questions (FAQ) about the NSFG. Hyattsville, MD.

National Center for Health Statistics. 2019. "About the National Survey of Family Growth.” Retrieved June 27, 2020 (https://www.cdc.gov/nchs/nsfg/about_nsfg.htm).

National Center for Health Statistics (NCHS). 2014. 2011-2013 National Survey of Family Growth Public-Use Data and Documentation. Hyattsville, MD: CDC National Center for Health Statistics.

National Center for Health Statistics (NCHS). 2016. 2013-2015 National Survey of Family Growth Public Use Data and Documentation. Hyattsville, MD: CDC National Center for Health Statistics.

National Center for Health Statistics (NCHS). 2018. 2015-2017 National Survey of Family Growth Public-Use Data and Documentation. Hyattsville, MD: CDC National Center for Health Statistics.

National Center for Health Statistics (NCHS). 2020. 2017-2019 National Survey of Family Growth Public-Use Data and Documentation. Hyattsville, MD: CDC National Center for Health Statistics.

Newlin Lew, Kelley, Caroline Dorsen, and Thomas Long. 2018. "Prevalence of Obesity, Prediabetes, and Diabetes in Sexual Minority Men: Results From the 2014 Behavioral Risk Factor Surveillance System.” The Diabetes Educator 44(1):83-93. doi: 10.1177/0145721717749943.

Nuttall, Frank Q. 2015. "Body Mass Index.” Nutrition Today 50(3):117-28. doi: 10.1097/NT.0000000000000092.

Ochs, Robyn. 1996. "Biphobia: It Goes More than Two Ways.” Pp. 217-39 in Bisexuality: The psychology and politics of an invisible minority. Thousand Oaks, CA, US: Sage Publications, Inc.

Office of the Surgeon General (US). 2010. "Background on Obesity." in The Surgeon General's Vision for a Healthy and Fit Nation. Rockville (MD): Office of the Surgeon General (US).

Paschen-Wolff, Margaret M., Elizabeth Kelvin, Brooke E. Wells, Aimee N. C. Campbell, Nicholas A. Grosskopf, and Christian Grov. 2019. "Changing Trends in Substance Use and Sexual Risk Disparities among Sexual Minority Women as a Function of Sexual Identity, Behavior, and Attraction: Findings from the National Survey of Family Growth, 2002-2015." Archives of Sexual Behavior 48(4):1137-58. doi: 10.1007/s10508-018-1333-1.

Patterson, Paul Daniel, Charity G. Moore, Janice C. Probst, and Judith Ann Shinogle. 2004. "Obesity and Physical Inactivity in Rural America." The Journal of Rural Health 20(2):151-59. doi:

10.1111/j.1748-0361.2004.tb00022.x.

Phelan, Jo C., Bruce G. Link, and Parisa Tehranifar. 2010. "Social Conditions as Fundamental Causes of Health Inequalities: Theory, Evidence, and Policy Implications.” Journal of Health and Social Behavior 51(1_suppl):S28-40. doi: 10.1177/0022146510383498. 
Platt, Lisa F., Julia Kay Wolf, and Christopher P. Scheitle. 2018. "Patterns of Mental Health Care Utilization Among Sexual Orientation Minority Groups." Journal of Homosexuality 65(2):13553. doi: 10.1080/00918369.2017.1311552.

Plöderl, Martin, and Pierre Tremblay. 2015. "Mental Health of Sexual Minorities. A Systematic Review." International Review of Psychiatry 27(5):367-85. doi: 10.3109/09540261.2015.1083949.

Rao, J. N. K., and A. J. Scott. 1981. "The Analysis of Categorical Data from Complex Sample Surveys: Chi-Squared Tests for Goodness of Fit and Independence in Two-Way Tables." Journal of the American Statistical Association 76:221-30.

Rao, J. N. K., and A. J. Scott. 1984. “On Chi-Squared Tests for Multiway Contingency Tables with Cell Proportions Estimated from Survey Data." Annals of Statistics 12:46-60.

Reczek, Corinne, Hui Liu, and Russell Spiker. 2017. "Self-Rated Health at the Intersection of Sexual Identity and Union Status." Social Science Research 63:242-52. doi:

10.1016/j.ssresearch.2016.09.013.

Riggle, Ellen D. B., Sharon S. Rostosky, Whitney W. Black, and Danielle E. Rosenkrantz. 2017. "Outness, Concealment, and Authenticity: Associations with LGB Individuals' Psychological Distress and Well-Being." Psychology of Sexual Orientation and Gender Diversity 4(1):54-62. doi: $10.1037 /$ sgd0000202.

Roberts, Susan J., Eileen M. Stuart-Shor, and Rachel A. Oppenheimer. 2010. “Lesbians' Attitudes and Beliefs Regarding Overweight and Weight Reduction.” Journal of Clinical Nursing 19(1314):1986-94. doi: 10.1111/j.1365-2702.2009.03182.x.

Rushe, Dominic. 2020. "US Supreme Court Rules Employers Cannot Discriminate Against LGBTQ+ Workers." The Guardian. Retrieved October 12, 2020 (http://www.theguardian.com/law/2020/jun/15/supreme-court-civil-rights-law-lgbtq).

Russell, Stephen T., Anne K. Driscoll, and Nhan Truong. 2002. “Adolescent Same-Sex Romantic Attractions and Relationships: Implications for Substance Use and Abuse." American Journal of Public Health 92(2):198-202. doi: 10.2105/AJPH.92.2.198.

Savin-Williams, Ritch C., and Kenneth M. Cohen. 2007. "Development of Same-Sex Attracted Youth." Pp. 27-47 in The Health of Sexual Minorities: Public Health Perspectives on Lesbian, Gay, Bisexual and Transgender Populations, edited by I. H. Meyer and M. E. Northridge. Boston, MA: Springer US.

Savin-Williams, Ritch C., and Zhana Vrangalova. 2013. "Mostly Heterosexual As a Distinct Sexual Orientation Group: A Systematic Review of the Empirical Evidence." Developmental Review 33(1):58-88. doi: 10.1016/j.dr.2013.01.001.

Scheitle, Christopher P., and Julia Kay Wolf. 2018. "Religion and Sexual Identity Fluidity in a National Three-Wave Panel of U.S. Adults." Archives of Sexual Behavior 47(4):1085-94. doi: 10.1007/s10508-017-0979-4.

Schneeberger, Andres R., Michael F. Dietl, Kristina H. Muenzenmaier, Christian G. Huber, and Undine E. Lang. 2014. "Stressful Childhood Experiences and Health Outcomes in Sexual Minority 
Populations: A Systematic Review." Social Psychiatry and Psychiatric Epidemiology 49(9):1427-45. doi: 10.1007/s00127-014-0854-8.

Schnittker, Jason, and Valerio Bacak. 2014. "The Increasing Predictive Validity of Self-Rated Health." PLOS ONE 9(1):e84933. doi: 10.1371/journal.pone.0084933.

Smith, David M., and Gary J. Gates. 2001. Gay and Lesbian Families in the United States: Same-Sex Unmarried Partner Households. A Preliminary Analysis of 2000 United States Census Data. Human Rights Campaign, 919 18th St.

Solazzo, Alexa, Bridget Gorman, and Justin Denney. 2020. "Does Sexual Orientation Complicate the Relationship Between Marital Status and Gender With Self-Rated Health and Cardiovascular Disease?" Demography; Silver Spring 57(2):599-626. doi: http://dx.doi.org.www.libproxy.wvu.edu/10.1007/s13524-020-00857-9.

Soleimanpour, Samira, Sara Geierstanger, and Claire D. Brindis. 2017. "Adverse Childhood Experiences and Resilience: Addressing the Unique Needs of Adolescents." Academic Pediatrics 17(7):S10814. doi: 10.1016/j.acap.2017.01.008.

Solomon, C. G., and J. E. Manson. 1997. "Obesity and Mortality: A Review of the Epidemiologic Data." The American Journal of Clinical Nutrition 66(4):1044S-1050S. doi: 10.1093/ajen/66.4.1044S.

stata.com. n.d. Svy: Tabulate Twoway - Two-Way Tables for Survey Data.

Stoddard, Sarah A., Jennifer Pierce, Noelle M. Hurd, José A. Bauermeister, and Marc A. Zimmerman. 2019. "Purpose and Mastery as Predictors of Perceived Health and Substance Use Problems." Journal of Community Psychology 47:1514-29. doi: 10.1002/jcop.22200.

Stracqualursi, Veronica. 2021. "Equality Act: Two GOP Lawmakers Who Supported the Legislation in 2019 Changed Their Votes This Time." CNN Politics. Retrieved February 26, 2021 (https://www.cnn.com/2021/02/26/politics/gop-lawmakers-equality-act/index.html).

Strutz, Kelly L., Amy H. Herring, and Carolyn Tucker Halpern. 2015. "Health Disparities Among Young Adult Sexual Minorities in the U.S." American Journal of Preventive Medicine 48(1):76-88. doi: 10.1016/j.amepre.2014.07.038.

Szymanski, Dawn M. 2005. "Heterosexism and Sexism as Correlates of Psychological Distress in Lesbians." Journal of Counseling \& Development 83(3):355-60. doi: 10.1002/j.15566678.2005.tb00355.x.

Szymanski, Dawn M., and Y. Barry Chung. 2001. "The Lesbian Internalized Homophobia Scale." Journal of Homosexuality 41(2):37-52. doi: 10.1300/J082v41n02_03.

Szymanski, Dawn M., Susan Kashubeck-West, and Jill Meyer. 2008. “Internalized Heterosexism Measurement, Psychosocial Correlates, and Research Directions.” The Counseling Psychologist 36(4):525-74. doi: 10.1177/0011000007309489.

Thoits, Peggy A. 1995. "Stress, Coping, and Social Support Processes: Where Are We? What Next?" Journal of Health and Social Behavior (Extra Issue: Forty Years of Medical Sociology: The State of the Art and Directions for the Future):53-79. 
Townsend, Megan. 2019. “GLAAD's 'Where We Are on Tv' Report Shows Tv Is Telling More LGBTQ Stories Than Ever." GLAAD. Retrieved February 25, 2021 (https:/www.glaad.org/blog/glaadswhere-we-are-tv-report-shows-tv-telling-more-lgbtq-stories-ever).

Tuthill, Zelma, Justin T. Denney, and Bridget Gorman. 2020. "Racial Disparities in Health and Health Behaviors Among Gay, Lesbian, Bisexual and Heterosexual Men and Women in the BRFSSSOP.” Ethnicity \& Health 25(2):177-88. doi: 10.1080/13557858.2017.1414157.

Twenge, Jean M., Ryne A. Sherman, and Brooke E. Wells. 2016. "Changes in American Adults' Reported Same-Sex Sexual Experiences and Attitudes, 1973-2014." Archives of Sexual Behavior 45(7):1713-30. doi: 10.1007/s10508-016-0769-4.

Umberson, Debra, and Jennifer Karas Montez. 2010. "Social Relationships and Health: A Flashpoint for Health Policy." Journal of Health and Social Behavior 51(1 Suppl):S54-66. doi: $10.1177 / 0022146510383501$.

US Department of Health \& Human Services. 2001. "The Surgeon General's Call To Action To Prevent and Decrease Overweight and Obesity 2001."

U.S. Department of Health and Human Services. 2020. 2017-2019 NSFG User's Guide Appendix 7c: Further Details on Variables Modified or Created for the Public-Use Files. Hyattsville, MD: Centers for Disease Control and Prevention.

Ward, Brian W., James M. Dahlhamer, Adena M. Galinsky, and Sarah S. Joestl. 2014. Sexual Orientation and Health Among U.S. Adults: National Health Interview Survey, 2013. 77. Hyattsville, MD: National Center for Health Statistics.

Warren, Jacob C., K. Bryant Smalley, and K. Nikki Barefoot. 2016. "Differences in Psychosocial Predictors of Obesity Among LGBT Subgroups." LGBT Health 3(4):283-91. doi: 10.1089/lgbt.2015.0076.

Wolff, Margaret, Brooke Wells, Christina Ventura-DiPersia, Audrey Renson, and Christian Grov. 2017. "Measuring Sexual Orientation: A Review and Critique of U.S. Data Collection Efforts and Implications for Health Policy." The Journal of Sex Research 54(4-5):507-31. doi: 10.1080/00224499.2016.1255872.

Wood, Mitchell J. 2004. “The Gay Male Gaze.” Journal of Gay \& Lesbian Social Services 17(2):43-62. doi: 10.1300/J041v17n02_03.

Woodard, Colin. 2011. American Nations: A History of the Eleven Rival Regional Cultures of North America. New York: Viking.

Woodell, Brandi. 2018. "Understanding Sexual Minority Health Disparities in Rural Areas." Sociology Compass 12(2):e12553. doi: https://doi.org/10.1111/soc4.12553.

Worthen, Meredith G. F. 2013. “An Argument for Separate Analyses of Attitudes Toward Lesbian, Gay, Bisexual Men, Bisexual Women, MtF and FtM Transgender Individuals.” Sex Roles 68:703-23.

Yean, Chetra, Erik Benau, Antonios Dakanalis, Julia M. Hormes, Julie Perone, and Alix Timko. 2013. "The Relationship of Sex and Sexual Orientation to Self-Esteem, Body Shape Satisfaction, and Eating Disorder Symptomatology." Frontiers in Psychology 4. doi: 10.3389/fpsyg.2013.00887. 


\section{APPENDIX}

TABLE 1-A. SUMMARY STATISTICS: IDENTITY

Table 1-A. Characteristics of US Adults Aged 25-45 Years, by Sexual Identity 2011-2019 (N=23,567)

\begin{tabular}{|c|c|c|c|c|c|c|}
\hline & \multicolumn{6}{|c|}{ Sexual Identity } \\
\hline & \multicolumn{2}{|c|}{$\begin{array}{l}\text { Heterosexual or straight } \\
\quad(\mathrm{n}=21,998 ; 93.3 \%)^{\mathrm{a}}\end{array}$} & \multicolumn{2}{|c|}{$\begin{array}{c}\text { Homosexual, gay, or } \\
\text { lesbian } \\
(\mathrm{n}=552 ; 2.3 \%)^{\mathrm{a}}\end{array}$} & \multicolumn{2}{|c|}{$\begin{array}{c}\text { Bisexual } \\
(\mathrm{n}=1,017 ; 4.3 \%)^{\mathrm{a}}\end{array}$} \\
\hline & Mean \% & $95 \%$ CI & Mean \% & $95 \% \mathrm{CI}$ & Mean \% & $95 \%$ CI \\
\hline \multicolumn{7}{|l|}{ Sexual Attraction*** } \\
\hline Only attracted to opposite sex & 92.2 & $(91.6,92.8)$ & 3.5 & $(1.7,6.9)$ & 5.1 & $(3.5,7.5)$ \\
\hline Mostly attracted to opposite sex & 7.3 & $(6.7,8.0)$ & 0.5 & $(0.2,1.3)$ & 41.8 & $(37.2,46.5)$ \\
\hline Equally attracted to males \& females & 0.4 & $(0.3,0.5)$ & 2.0 & $(1.1,3.5)$ & 45.9 & $(41.5,50.4)$ \\
\hline Mostly attracted to same sex & 0.0 & $(0.0,0.0)$ & 27.6 & $(22.0,34.0)$ & 6.9 & $(5.1,9.4)$ \\
\hline Only attracted to same sex & 0.1 & $(0.0,0.1)$ & 66.5 & $(60.1,72.3)$ & 0.2 & $\left(\begin{array}{ll}0.1 & 0.9\end{array}\right)$ \\
\hline \multicolumn{7}{|l|}{ Sexual Behavior**** } \\
\hline Sexual experience with same-sex partner & 7.5 & $(7.0,8.1)$ & 96.0 & $(93.3,97.7)$ & 82.8 & $(78.8,86.2)$ \\
\hline No sexual experience with same-sex partner & 92.5 & $(91.9,93.0)$ & 4.0 & $(2.4,6.7)$ & 17.2 & $(13.8,21.3)$ \\
\hline \multicolumn{7}{|l|}{ Sex $* * *$} \\
\hline Female & 48.2 & $(47.2,49.2)$ & 46.9 & $(40.4,53.4)$ & 77.7 & $(73.8,81.2)$ \\
\hline Male & 51.8 & $(50.8,52.8)$ & 53.2 & $(46.6,59.6)$ & 22.3 & $(18.8,26.2)$ \\
\hline \multicolumn{7}{|l|}{ Age $^{* * *}$} \\
\hline 25-34 & 50.8 & $(49.7,51.9)$ & 58.6 & $(52.2,64.8)$ & 65.3 & $(61.3,69.2)$ \\
\hline $35-44$ & 49.2 & $(48.1,50.4)$ & 41.4 & $(35.3,47.8)$ & 34.7 & $(30.8,38.7)$ \\
\hline \multicolumn{7}{|l|}{ Race/ethnicity } \\
\hline White, non-Hispanic & 57.9 & $(55.8,60.0)$ & 55.6 & $(48.7,62.4)$ & 63.3 & $(58.7,67.8)$ \\
\hline Hispanic & 19.8 & $(18.0,21.8)$ & 20.9 & $(15.1,28.1)$ & 14.8 & $(11.9,18.3)$ \\
\hline Black, non-Hispanic & 12.2 & $(11.0,13.6)$ & 11.2 & $(8.0,15.5)$ & 12.1 & $(9.7,15.0)$ \\
\hline Other, non-Hispanic & 10.0 & $(9.0,11.1)$ & 12.3 & $(8.9,16.6)$ & 9.7 & $(6.7,13.8)$ \\
\hline \multicolumn{7}{|l|}{ Residence** $^{* *}$} \\
\hline Principal MSA city & 33.7 & $(30.9,36.6)$ & 44.6 & $(37.3,52.2)$ & 39.4 & $(34.6,44.5)$ \\
\hline Other MSA & 50.5 & $(47.4,53.6)$ & 42.8 & $(35.2,50.8)$ & 47.3 & $(42.6,52.2)$ \\
\hline Not MSA & 15.9 & $(12.9,19.4)$ & 12.6 & $(8.4,18.4)$ & 13.2 & $(9.4,18.3)$ \\
\hline \multicolumn{7}{|l|}{ Nativity*** } \\
\hline US-born & 81.6 & $(80.3,82.8)$ & 86.4 & $(81.2,90.4)$ & 91.9 & $(89.3,93.9)$ \\
\hline Foreign-born & 18.4 & $(17.2,19.8)$ & 13.6 & $(9.6,18.9)$ & 8.1 & $(6.1,10.7)$ \\
\hline \multicolumn{7}{|l|}{ Education } \\
\hline Some high school or less & 10.2 & $(9.4,11.1)$ & 6.7 & $(4.3,10.3)$ & 9.1 & $(7.1,11.7)$ \\
\hline High school diploma or GED & 25.3 & $(24.2,26.5)$ & 23.3 & $(18.4,29.2)$ & 25.6 & $(21.7,30.1)$ \\
\hline Some college & 19.5 & $(18.6,20.5)$ & 20.6 & $(16.2,25.9)$ & 22.6 & $(19.2,26.5)$ \\
\hline College or graduate degree & 44.9 & $(43.0,46.8)$ & 49.3 & $(43.5,55.2)$ & 42.6 & $(37.8,47.6)$ \\
\hline \multicolumn{7}{|l|}{ Insurance $* * *$} \\
\hline Private or Medi-Gap & 64.8 & $(63.2,66.4)$ & 65.3 & $(59.3,70.8)$ & 52.8 & $(48.6,57.1)$ \\
\hline Medicaid, CHIP, State-sponsored & 11.8 & $(10.9,12.8)$ & 12.8 & $(9.6,17.0)$ & 21.6 & $(18.6,25.0)$ \\
\hline Medicare, military, government & 4.7 & $(3.9,5.5)$ & 5.0 & $(3.2,7.8)$ & 4.4 & $(3.1,6.3)$ \\
\hline Single-service, Indian Health Service, not covered & 18.7 & $(17.5,19.9)$ & 16.9 & $(12.6,22.2)$ & 21.1 & $(17.7,25.0)$ \\
\hline \multicolumn{7}{|l|}{ Household income, \% of federal poverty level $* * *$} \\
\hline $0-99$ & 17.7 & $(16.7,18.8)$ & 19.7 & $(15.0,25.5)$ & 23.8 & $(20.6,27.4)$ \\
\hline 100-299 & 36.5 & $(35.2,37.9)$ & 32.3 & $(26.8,38.4)$ & 42.4 & $(38.2,46.6)$ \\
\hline$>\mathbf{3 0 0} \%$ & 45.8 & $(44.0,47.6)$ & 48.0 & $(41.0,55.0)$ & 33.8 & $(29.5,38.5)$ \\
\hline Self-rated health $* * *$ & & & & & & \\
\hline Poor & 1.1 & $(0.9,1.3)$ & 3.3 & $(1.4,7.9)$ & 1.8 & $(1.2,2.7)$ \\
\hline Fair & 6.6 & $(6.1,7.1)$ & 6.3 & $(4.4,8.8)$ & 10.7 & $(8.6,13.3)$ \\
\hline Good & 25.5 & $(24.6,26.4)$ & 25.3 & $(20.8,30.4)$ & 34.5 & $(30.2,39.0)$ \\
\hline Very good & 39.5 & $(38.5,40.5)$ & 34.1 & $(28.6,40.1)$ & 32.0 & $(27.9,36.4)$ \\
\hline Excellent & 27.3 & $(26.4,28.3)$ & 30.9 & $(25.5,36.9)$ & 21.1 & $(17.8,24.7)$ \\
\hline BMI* & & & & & & \\
\hline <18.5 (Underweight) & 1.1 & $(0.9,1.4)$ & 0.7 & $(0.3,1.7)$ & 0.7 & $(0.3,1.3)$ \\
\hline 18.5-24.9 (Normal) & 32.7 & $(31.6,33.7)$ & 38.7 & $(32.8,45.0)$ & 33.2 & $(28.9,37.9)$ \\
\hline 25.0-29.9 (Overweight) & 33.9 & $(32.9,34.8)$ & 28.7 & $(23.4,34.7)$ & 28.5 & $(24.6,32.7)$ \\
\hline$>30.0$ (Obese) & 32.4 & $(31.3,33.5)$ & 31.9 & $(26.9,37.3)$ & 37.7 & $(33.4,42.1)$ \\
\hline Access to Usual Source of Care & & & & & & \\
\hline Yes & 78.7 & $(77.7,79.7)$ & 84.0 & $(78.7,88.3)$ & 78.9 & $(77.4,84.5)$ \\
\hline No & 21.3 & $(20.3,22.3)$ & 16.0 & $(11.8,21.3)$ & 21.1 & $(15.5,22.6)$ \\
\hline Cycle*** & & & & & & \\
\hline 2011-2013 & 25.3 & $(23.3,27.4)$ & 18.3 & $(13.7,24.0)$ & 22.3 & $(18.6,26.6)$ \\
\hline 2013-2015 & 26.7 & $(24.5,29.0)$ & 21.1 & $(16.6,26.4)$ & 22.4 & $(18.6,26.8)$ \\
\hline 2015-2017 & 24.3 & $(22.2,26.5)$ & 33.7 & $(25.1,43.5)$ & 23.3 & $(19.7,27.4)$ \\
\hline 2017-2019 & 23.7 & $(21.6,26.0)$ & 27.0 & $(21.2,33.6)$ & 31.9 & $(27.1,37.2)$ \\
\hline
\end{tabular}

${ }^{\mathrm{a}} \mathrm{n}=$ sample number and percent without using svy command; Mean and $95 \%$ CI were conducted using svy command.

${ }^{*} \mathrm{p}<0.05, * * \mathrm{p}<0.01,{ }^{* * *} \mathrm{p}<0.001 ; p$-values were determined using the "Pearson $\chi 2$ test with the Rao and Scott $(1981,1984)$ second-order correction" or the $F$ test (the default output from the Stata svy tabulate command) (Stata.com n.d.:11).

Note. Sample does not include pregnant females or those missing values listwise on these variables. MSA = metropolitan statistical area; $\mathrm{BMI}=$ body mass index; not all strata included in each cell if missing subpopulation members. 
TABLE 1-B. SUMMARY STATISTICS: ATTRACTION

Table 1-B. Characteristics of US Adults Aged 25-45 Years, by Sexual Attraction 2011-2019 (N=23,567)

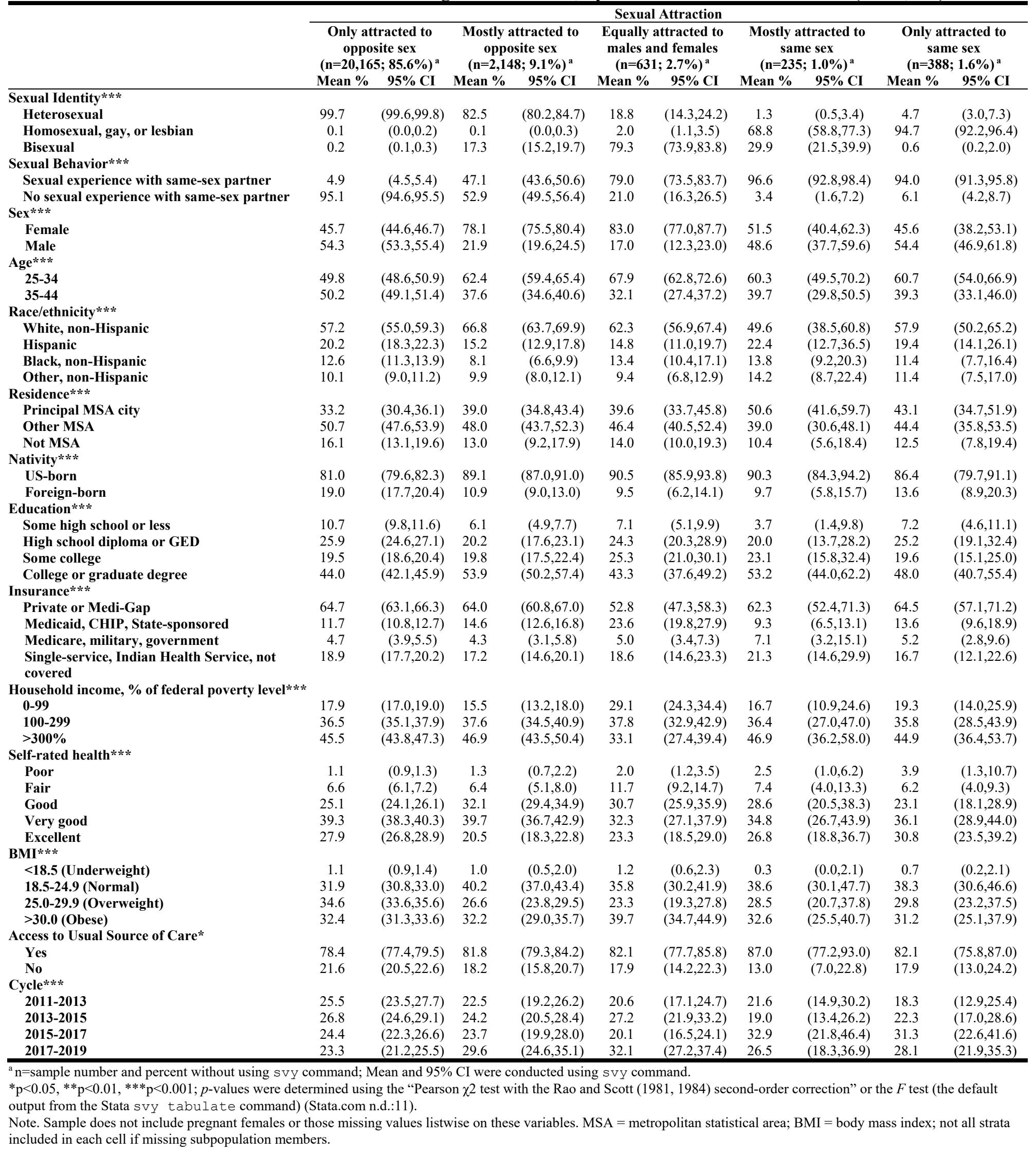


TABLE 1-C. SUMMARY STATISTICS: BEHAVIOR

Table 1-C. Characteristics of US Adults Aged 25-45 Years, by Sexual Behavior 2011-2019 (N=23,567)

\begin{tabular}{|c|c|c|c|c|}
\hline & \multicolumn{4}{|c|}{ Sexual Behavior } \\
\hline & \multicolumn{2}{|c|}{$\begin{array}{c}\text { Sexual experience } \\
\text { with same sex } \\
\text { partner } \\
(\mathbf{n}=\mathbf{3}, \mathbf{2 8 2} ; \mathbf{1 3 . 9 \%})^{\mathrm{a}}\end{array}$} & \multicolumn{2}{|c|}{$\begin{array}{c}\text { No sexual experience } \\
\text { with same sex } \\
\text { partner } \\
(\mathbf{n}=\mathbf{2 0 , 2 8 5} ; \mathbf{8 6 . 1 \%})^{\mathrm{a}}\end{array}$} \\
\hline & Mean \% & $95 \%$ CI & Mean \% & $95 \%$ CI \\
\hline \multicolumn{5}{|l|}{ Sexual Identity*** } \\
\hline Heterosexual & 59.8 & $(57.1,62.3)$ & 99.2 & $(99.1,99.4)$ \\
\hline Homosexual, gay, or lesbian & 16.2 & $(14.0,18.5)$ & 0.1 & $(0.1,0.2)$ \\
\hline Bisexual & 24.1 & $(22.1,26.2)$ & 0.7 & $(0.5,0.9)$ \\
\hline \multicolumn{5}{|l|}{ Sexual Attraction $* * *$} \\
\hline Only attracted to opposite sex & 36.1 & $(33.7,38.6)$ & 94.4 & $(93.7,94.9)$ \\
\hline Mostly attracted to opposite sex & 33.0 & $(30.7,35.4)$ & 5.0 & $(4.5,5.6)$ \\
\hline Equally attracted to males \& females & 13.3 & $(11.8,15.0)$ & 0.5 & $(0.4,0.6)$ \\
\hline Mostly attracted to same sex & 6.5 & $(5.3,8.0)$ & 0.0 & $(0.0,0.1)$ \\
\hline Only attracted to same sex & 11.1 & $(9.5,12.9)$ & 0.1 & $(0.1,0.1)$ \\
\hline \multicolumn{5}{|l|}{ Sex $* * *$} \\
\hline Female & 74.6 & $(72.2,76.9)$ & 45.7 & $(44.7,46.7)$ \\
\hline Male & 25.4 & $(23.1,27.8)$ & 54.3 & $(53.3,55.3)$ \\
\hline \multicolumn{5}{|l|}{$\operatorname{Age}^{* * *}$} \\
\hline $25-34$ & 56.9 & $(54.3,59.4)$ & 50.7 & $(49.5,51.8)$ \\
\hline $35-44$ & 43.1 & $(40.6,45.7)$ & 49.3 & $(48.2,50.5)$ \\
\hline \multicolumn{5}{|l|}{ Race/ethnicity*** } \\
\hline White, non-Hispanic & 63.8 & $(61.0,66.5)$ & 57.3 & $(55.1,59.4)$ \\
\hline Hispanic & 15.3 & $(13.2,17.6)$ & 20.3 & $(18.3,22.4)$ \\
\hline Black, non-Hispanic & 12.3 & $(10.5,14.3)$ & 12.2 & $(11.0,13.5)$ \\
\hline Other, non-Hispanic & 8.6 & $(7.3,10.2)$ & 10.3 & $(9.2,11.5)$ \\
\hline \multicolumn{5}{|l|}{ Residence** } \\
\hline Principal MSA city & 38.8 & $(35.1,42.7)$ & 33.4 & $(30.6,36.3)$ \\
\hline Other MSA & 46.0 & $(42.1,49.9)$ & 50.8 & $(47.7,53.9)$ \\
\hline Not MSA & 15.2 & $(11.5,19.8)$ & 15.8 & $(12.8,19.3)$ \\
\hline \multicolumn{5}{|l|}{ Nativity*** } \\
\hline US-born & 92.3 & $(90.8,93.6)$ & 80.6 & $(79.2,82.0)$ \\
\hline Foreign-born & 7.7 & $(6.4,9.2)$ & 19.4 & $(18.0,20.8)$ \\
\hline \multicolumn{5}{|l|}{ Education $* * * *$} \\
\hline Some high school or less & 6.6 & $(5.6,7.8)$ & 10.6 & $(9.7,11.5)$ \\
\hline High school diploma or GED & 24.5 & $(22.1,27.1)$ & 25.4 & $(24.2,26.7)$ \\
\hline Some college & 24.0 & $(21.8,26.3)$ & 19.1 & $(18.2,20.0)$ \\
\hline College or graduate degree & 44.9 & $(41.8,48.1)$ & 44.9 & $(43.0,46.8)$ \\
\hline \multicolumn{5}{|l|}{ Insurance $* * *$} \\
\hline Private or Medi-Gap & 58.2 & $(55.3,61.1)$ & 65.3 & $(63.6,66.8)$ \\
\hline Medicaid, CHIP, State-sponsored & 18.1 & $(16.2,20.1)$ & 11.4 & $(10.5,12.4)$ \\
\hline Medicare, military, government & 5.1 & $(4.1,6.4)$ & 4.6 & $(3.8,5.5)$ \\
\hline Single-service, Indian Health Service, not covered & 18.6 & $(16.5,20.9)$ & 18.8 & $(17.5,20.0)$ \\
\hline \multicolumn{5}{|l|}{ Household income, $\%$ of federal poverty level $* * *$} \\
\hline 0-99 & 21.6 & $(19.5,23.9)$ & 17.5 & $(16.5,18.5)$ \\
\hline 100-299 & 38.6 & $(36.0,41.3)$ & 36.3 & $(35.0,37.7)$ \\
\hline$>\mathbf{3 0 0} \%$ & 39.7 & $(36.9,42.6)$ & 46.2 & $(44.4,48.0)$ \\
\hline \multicolumn{5}{|l|}{ Self-rated health*** } \\
\hline Poor & 2.1 & $(1.5,3.0)$ & 1.0 & $(0.8,1.2)$ \\
\hline Fair & 9.0 & $(7.8,10.4)$ & 6.4 & $(5.9,7.0)$ \\
\hline Good & 31.5 & $(29.2,33.9)$ & 25.0 & $(24.1,26.0)$ \\
\hline Very good & 35.2 & $(32.9,37.5)$ & 39.7 & $(38.7,40.7)$ \\
\hline Excellent & 22.2 & $(20.2,24.4)$ & 27.9 & $(26.8,28.9)$ \\
\hline \multicolumn{5}{|l|}{ BMI**** } \\
\hline$<18.5$ (Underweight) & 0.8 & $(0.5,1.3)$ & 1.1 & $(0.9,1.4)$ \\
\hline 18.5-24.9 (Normal) & 35.6 & $(33 \cdot 0,38.3)$ & 32.4 & $(31.3,33.5)$ \\
\hline 25.0-29.9 (Overweight) & 28.3 & $(26.1,30.7)$ & 34.3 & $(33.3,35.3)$ \\
\hline$>30.0$ (Obese) & 35.2 & $(32.8,37.7)$ & 32.2 & $(31.1,33.3)$ \\
\hline Access to Usual Source of Care $* * *$ & & & & \\
\hline Yes & 84.2 & $(82.3,85.9)$ & 78.2 & $(77.1,79.3)$ \\
\hline No & 15.8 & $(14.1,17.7)$ & 21.8 & $(20.7,22.9)$ \\
\hline Cycle* & & & & \\
\hline 2011-2013 & 22.9 & $(20.3,25.7)$ & 25.3 & $(23.2,27.6)$ \\
\hline 2013-2015 & 24.9 & $(22.1,27.9)$ & 26.6 & $(24.4,29.0)$ \\
\hline 2015-2017 & 24.8 & $(21.8,28.1)$ & 24.4 & $(22.2,26.7)$ \\
\hline 2017-2019 & 27.4 & $(24.1,30.9)$ & 23.6 & $(21.4,26.0)$ \\
\hline
\end{tabular}

${ }^{a} \mathrm{n}=$ sample number and percent without using svy command; Mean and $95 \% \mathrm{CI}$ were conducted using svy command. ${ }^{*} \mathrm{p}<0.05,{ }^{* *} \mathrm{p}<0.01,{ }^{* * *} \mathrm{p}<0.001 ; p$-values were determined using the "Pearson $\chi 2$ test with the Rao and Scott (1981, 1984) second-order correction" or the $F$ test (the default output from the Stata svy tabulate command) (Stata.com n.d.:11).

Note. Sample does not include pregnant females or those missing values listwise on these variables. MSA = metropolitan statistical area; BMI = body mass index; not all strata included in each cell if missing subpopulation members. 
Table 1-D. Sample Characteristics of US Adults by Sexual Identity Components and Health Outcomes 2011-2019 (N=23,567)

\begin{tabular}{|c|c|c|c|c|c|c|c|c|}
\hline & \multirow[b]{2}{*}{$\begin{array}{l}\text { Number in } \\
\text { thousands }\end{array}$} & \multicolumn{7}{|c|}{ Self-Rated Health } \\
\hline & & $\begin{array}{c}\% \text { of Total } \\
\text { Sample }\end{array}$ & Poor & Fair & Good & Very Good & Excellent & $\begin{array}{c}* \text { Outcome sig. } \\
\text { differences }\end{array}$ \\
\hline Total Adult Sample & 23,567 & 100.0 & $1.4 \%$ & $7.6 \%$ & $26.6 \%$ & $38.1 \%$ & $26.3 \%$ & \\
\hline \multicolumn{9}{|l|}{ Sexual Identity } \\
\hline Heterosexual $^{\mathrm{A}}$ & 21,998 & 93.3 & $1.3 \%$ & $7.4 \%$ & $26.2 \%$ & $38.5 \%$ & $26.5 \%$ & $\mathrm{~B}, \mathrm{C}$ \\
\hline Homosexual, gay, or lesbian ${ }^{B}$ & 552 & 2.3 & $2.7 \%$ & $8.9 \%$ & $28.1 \%$ & $34.6 \%$ & $25.7 \%$ & A \\
\hline Bisexual $^{\mathrm{C}}$ & 1,017 & 4.3 & $2.9 \%$ & $12.2 \%$ & $33.5 \%$ & $30.7 \%$ & $20.8 \%$ & A \\
\hline \multicolumn{9}{|l|}{ Sexual Behavior } \\
\hline No sexual experience with same-sex partner ${ }^{A}$ & 20,285 & 86.1 & $1.2 \%$ & $7.2 \%$ & $25.7 \%$ & $38.7 \%$ & $27.2 \%$ & $\mathrm{~B}$ \\
\hline Sexual experience with same-sex partner $^{B}$ & 3,282 & 13.9 & $2.5 \%$ & $10.5 \%$ & $31.8 \%$ & $34.6 \%$ & $20.5 \%$ & A \\
\hline \multicolumn{9}{|l|}{ Sexual Attraction } \\
\hline Only attracted to opposite $\operatorname{sex}^{\mathrm{A}}$ & 20,165 & 85.6 & $1.3 \%$ & $7.4 \%$ & $25.7 \%$ & $38.4 \%$ & $27.2 \%$ & $\mathrm{C}$ \\
\hline Mostly attracted to opposite $\operatorname{sex}^{B}$ & 2,148 & 9.1 & $1.4 \%$ & $8.2 \%$ & $31.8 \%$ & $38.3 \%$ & $20.3 \%$ & $\mathrm{C}$ \\
\hline Equally attracted to males \& females ${ }^{\mathrm{C}}$ & 631 & 2.7 & $2.7 \%$ & $13.6 \%$ & $34.4 \%$ & $30.0 \%$ & $19.3 \%$ & $\mathrm{~A}, \mathrm{~B}, \mathrm{E}$ \\
\hline Mostly attracted to same $\operatorname{sex}^{D}$ & 235 & 1.0 & $3.4 \%$ & $7.7 \%$ & $29.8 \%$ & $34.9 \%$ & $24.3 \%$ & \\
\hline Only attracted to same $\operatorname{sex}^{\mathrm{E}}$ & 388 & 1.6 & $2.6 \%$ & $8.8 \%$ & $26.8 \%$ & $36.6 \%$ & $25.3 \%$ & $\mathrm{C}$ \\
\hline \multicolumn{9}{|l|}{ Sex } \\
\hline Female $^{\mathrm{A}}$ & 12,895 & 54.7 & $1.6 \%$ & $8.6 \%$ & $27.3 \%$ & $36.8 \%$ & $25.8 \%$ & $\mathrm{~B}$ \\
\hline Male $^{B}$ & 10,672 & 45.3 & $1.1 \%$ & $6.5 \%$ & $25.8 \%$ & $39.7 \%$ & $26.9 \%$ & A \\
\hline \multicolumn{9}{|l|}{ Age Group } \\
\hline $25-34^{\mathrm{A}}$ & 13,038 & 55.3 & $1.0 \%$ & $6.3 \%$ & $25.6 \%$ & $38.4 \%$ & $28.6 \%$ & B \\
\hline \multirow[t]{3}{*}{$35-44^{B}$} & 10,529 & 44.7 & $1.9 \%$ & $9.3 \%$ & $27.8 \%$ & $37.7 \%$ & $23.4 \%$ & A \\
\hline & & \multicolumn{7}{|c|}{ Body Mass Index (BMI) } \\
\hline & $\begin{array}{l}\text { Number in } \\
\text { thousands }\end{array}$ & $\begin{array}{l}\text { \% of Total } \\
\text { Sample }\end{array}$ & $\begin{array}{c}<18.5 \\
\text { (Underweight) }\end{array}$ & $\begin{array}{c}20-24 \\
\text { (Normal) }\end{array}$ & $\begin{array}{c}25.0-29.9 \\
\text { (Overweight) }\end{array}$ & $\begin{array}{c}30-50 \\
\text { (Obese) }\end{array}$ & & $\begin{array}{c}\text { *Outcome sig. } \\
\text { differences }\end{array}$ \\
\hline Total Adult Sample & 23,567 & 100.0 & $1.1 \%$ & $32.1 \%$ & $31.2 \%$ & $35.0 \%$ & & \\
\hline \multicolumn{9}{|l|}{ Sexual Identity } \\
\hline Heterosexual $^{\mathrm{A}}$ & 21,998 & 93.3 & $1.1 \%$ & $32.1 \%$ & $32.0 \%$ & $34.7 \%$ & & $\mathrm{C}$ \\
\hline Homosexual, gay, or lesbian ${ }^{B}$ & 552 & 2.3 & $1.1 \%$ & $35.5 \%$ & $29.4 \%$ & $34.1 \%$ & & $\mathrm{C}$ \\
\hline Bisexual $^{\mathrm{C}}$ & 1,017 & 4.3 & $1.0 \%$ & $30.3 \%$ & $27.5 \%$ & $41.2 \%$ & & $\mathrm{~A}, \mathrm{~B}$ \\
\hline \multicolumn{9}{|l|}{ Sexual Behavior } \\
\hline No sexual experience with same-sex partner ${ }^{A}$ & 20,285 & 86.1 & $1.1 \%$ & $32.0 \%$ & $32.4 \%$ & $34.5 \%$ & & B \\
\hline Sexual experience with same-sex partner ${ }^{B}$ & 3,282 & 13.9 & $1.1 \%$ & $33.0 \%$ & $28.1 \%$ & $37.8 \%$ & & A \\
\hline Sexual Attraction & & & & & & & & \\
\hline Only attracted to opposite $\operatorname{sex}^{\mathrm{A}}$ & 20,165 & 85.6 & $1.1 \%$ & $31.7 \%$ & $32.7 \%$ & $34.5 \%$ & & $\mathrm{~B}, \mathrm{C}$ \\
\hline Mostly attracted to opposite $\operatorname{sex}^{B}$ & 2,148 & 9.1 & $1.2 \%$ & $35.7 \%$ & $27.0 \%$ & $36.2 \%$ & & $\mathrm{~A}, \mathrm{C}$ \\
\hline Equally attracted to males \& females ${ }^{C}$ & 631 & 2.7 & $1.6 \%$ & $30.0 \%$ & $23.0 \%$ & $45.5 \%$ & & $\mathrm{~A}, \mathrm{~B}, \mathrm{D}, \mathrm{E}$ \\
\hline Mostly attracted to same $\operatorname{sex}^{D}$ & 235 & 1.0 & $0.4 \%$ & $34.9 \%$ & $30.2 \%$ & $34.5 \%$ & & $\mathrm{C}$ \\
\hline Only attracted to same $\operatorname{sex}^{\mathrm{E}}$ & 388 & 1.6 & $1.0 \%$ & $36.6 \%$ & $28.9 \%$ & $33.5 \%$ & & $\mathrm{C}$ \\
\hline Sex & & & & & & & & \\
\hline Female $^{\mathrm{A}}$ & 12,895 & 54.7 & $1.4 \%$ & $35.2 \%$ & $25.7 \%$ & $37.7 \%$ & & B \\
\hline Male $^{\text {B }}$ & 10,672 & 45.3 & $0.8 \%$ & $28.4 \%$ & $39.2 \%$ & $31.6 \%$ & & A \\
\hline Age Group & & & & & & & & \\
\hline $25-34^{\mathrm{A}}$ & 13,038 & 55.3 & $1.4 \%$ & $35.2 \%$ & $30.8 \%$ & $32.7 \%$ & & $\mathrm{~B}$ \\
\hline $35-44^{B}$ & 10,529 & 44.7 & $0.8 \%$ & $28.3 \%$ & $33.1 \%$ & $37.8 \%$ & & $\mathrm{~A}$ \\
\hline & & & Access to Us & al Source o & Healthcare & & & \\
\hline & $\begin{array}{l}\text { Number in } \\
\text { thousands }\end{array}$ & $\begin{array}{c}\% \text { of Total } \\
\text { Sample }\end{array}$ & Yes & No & & & & $\begin{array}{c}\text { *Outcome sig. } \\
\text { differences }\end{array}$ \\
\hline Total Adult Sample & 23,567 & 100.0 & $78.7 \%$ & $21.3 \%$ & & & & \\
\hline Sexual Identity & & & & & & & & \\
\hline Heterosexual $^{\mathrm{A}}$ & 21,998 & 93.3 & $78.4 \%$ & $21.6 \%$ & & & & B \\
\hline Homosexual, gay, or lesbian ${ }^{B}$ & 552 & 2.3 & $83.0 \%$ & $17.0 \%$ & & & & A \\
\hline Bisexual $^{\mathrm{C}}$ & 1,017 & 4.3 & $80.9 \%$ & $19.1 \%$ & & & & \\
\hline Sexual Behavior & & & & & & & & \\
\hline No sexual experience with same-sex partner ${ }^{A}$ & 20,285 & 86.1 & $78.0 \%$ & $22.0 \%$ & & & & $\mathrm{~B}$ \\
\hline Sexual experience with same-sex partner ${ }^{B}$ & 3,282 & 13.9 & $82.5 \%$ & $17.6 \%$ & & & & A \\
\hline Sexual Attraction & & & & & & & & \\
\hline Only attracted to opposite $\operatorname{sex}^{\mathrm{A}}$ & 20,165 & 85.6 & $78.2 \%$ & $21.8 \%$ & & & & $\mathrm{~B}, \mathrm{C}, \mathrm{D}$ \\
\hline Mostly attracted to opposite $\operatorname{sex}^{B}$ & 2,148 & 9.1 & $80.9 \%$ & $19.1 \%$ & & & & A, D \\
\hline Equally attracted to males \& females ${ }^{\mathrm{C}}$ & 631 & 2.7 & $82.6 \%$ & $17.4 \%$ & & & & A \\
\hline Mostly attracted to same $\operatorname{sex}^{D}$ & 235 & 1.0 & $87.7 \%$ & $12.3 \%$ & & & & $\mathrm{~A}, \mathrm{~B}, \mathrm{E}$ \\
\hline Only attracted to same $\operatorname{sex}^{\mathrm{E}}$ & 388 & 1.6 & $79.6 \%$ & $20.4 \%$ & & & & D \\
\hline Sex & & & & & & & & \\
\hline Female $^{A}$ & 12,895 & 54.7 & $86.1 \%$ & $13.9 \%$ & & & & B \\
\hline Male $^{\text {B }}$ & 10,672 & 45.3 & $69.7 \%$ & $30.3 \%$ & & & & A \\
\hline Age Group & & & & & & & & \\
\hline $25-34^{\mathrm{A}}$ & 13,038 & 55.3 & $75.0 \%$ & $25.0 \%$ & & & & $\mathrm{~B}$ \\
\hline $35-44^{\mathrm{B}}$ & 10,529 & 44.7 & $83.2 \%$ & $16.9 \%$ & & & & A \\
\hline
\end{tabular}

Note. Table presents unweighted data analyses. Row totals may not equal 100.0 due to rounding.

This sample does not include pregnant females or those missing values listwise on these and other variables explored in later regression analyses.

*Items in same greyscale were grouped together in above Pearson $\chi^{2}$ tests due to small cell sizes and prior examples in the literature.

Superscripts denote a Pearson $\chi^{2}$ test of $p<0.05$ between that category and its corresponding superscript. Fisher's exact two-tailed test also run for $2 \times 2$ tables; results are consistent. 
Table 1-E. Regressions of Health Outcomes on Sexual Identity for Adults Age 25-45 (N=23,567)

\begin{tabular}{|c|c|c|c|c|c|c|c|c|}
\hline & \multicolumn{2}{|c|}{ Self-Rated Health } & \multicolumn{2}{|c|}{ Overweight } & \multicolumn{2}{|c|}{ Obese } & \multicolumn{2}{|c|}{ Usual Source of Care } \\
\hline & OR & 95\% CI & RRR & $95 \% \mathrm{CI}$ & RRR & $95 \% \mathrm{CI}$ & OR & $95 \%$ CI \\
\hline Intercept & $14.24 * * *$ & $(9.59,21.15)$ & $0.54 * * *$ & $(0.42,0.69)$ & $0.68 * *$ & $(0.53,0.87)$ & $4.32 * * *$ & $(3.30,5.67)$ \\
\hline Heterosexual (ref) & 1.0 & & 1.0 & & 1.0 & & 1.0 & \\
\hline Homosexual, gay, or lesbian & 0.77 & $(0.49,1.20)$ & 0.73 & $(0.51,1.04)$ & 0.85 & $(0.64,1.13)$ & 1.45 & $(0.98,2.13)$ \\
\hline Bisexual & $0.65 * *$ & $(0.51,0.83)$ & 1.12 & $(0.86,1.46)$ & $1.28 *$ & $(1.02,1.62)$ & 0.97 & $(0.75,1.25)$ \\
\hline 2011-2013 (ref) & 1.0 & & 1.0 & & 1.0 & & 1.0 & \\
\hline 2013-2015 & $0.77 * *$ & $(0.64,0.92)$ & $0.85^{*}$ & $(0.74,0.98)$ & 0.93 & $(0.81,1.06)$ & 0.97 & $(0.82,1.14)$ \\
\hline 2015-2017 & 0.85 & $(0.68,1.06)$ & 0.88 & $(0.77,1.02)$ & 0.97 & $(0.83,1.13)$ & 1.05 & $(0.89,1.25)$ \\
\hline 2017-2019 & $0.79 * *$ & $(0.66,0.94)$ & 1.01 & $(0.88,1.15)$ & 1.11 & $(0.94,1.31)$ & $0.82 *$ & $(0.69,0.99)$ \\
\hline \multicolumn{9}{|l|}{ Sex } \\
\hline Female (ref) & 1.0 & & 1.0 & & 1.0 & & 1.0 & \\
\hline Male & 1.05 & $(0.91,1.22)$ & $2.39 * * *$ & $(2.16,2.65)$ & $1.54 * * *$ & $(1.38,1.72)$ & $0.35 * * *$ & $(0.31,0.39)$ \\
\hline \multicolumn{9}{|l|}{ Age } \\
\hline $35-44$ & $0.54 * * *$ & $(0.47,0.63)$ & $1.43 * * *$ & $(1.30,1.57)$ & $1.68 * * *$ & $(1.52,1.86)$ & $1.63 * * *$ & $(1.46,1.83)$ \\
\hline \multicolumn{9}{|l|}{ Race/ethnicity } \\
\hline White, non-Hispanic (ref) & 1.0 & & 1.0 & & 1.0 & & 1.0 & \\
\hline Hispanic & $0.81 *$ & $(0.66,0.99)$ & $1.48 * * *$ & $(1.28,1.72)$ & $1.85 * * *$ & $(1.59,2.14)$ & 0.96 & $(0.82,1.11)$ \\
\hline Black, non-Hispanic & 1.07 & $(0.85,1.34)$ & $1.32 * * *$ & $(1.14,1.53)$ & $2.17 * * *$ & $(1.89,2.48)$ & $1.24 * *$ & $(1.06,1.46)$ \\
\hline Other, non-Hispanic & 0.84 & $(0.64,1.09)$ & $0.74 * *$ & $(0.63,0.88)$ & 0.88 & $(0.73,1.05)$ & $1.23 *$ & $(1.02,1.47)$ \\
\hline \multicolumn{9}{|l|}{ Nativity } \\
\hline US-born (ref) & 1.0 & & 1.0 & & 1.0 & & 1.0 & \\
\hline Foreign-born & $1.52 * * *$ & $(1.23,1.86)$ & $0.78 * *$ & $(0.68,0.90)$ & $0.44 * * *$ & $(0.38,0.51)$ & $0.79 * *$ & $(0.68,0.91)$ \\
\hline \multicolumn{9}{|l|}{ Residence } \\
\hline Principal MSA city (ref) & 1.0 & & 1.0 & & 1.0 & & 1.0 & \\
\hline Other MSA & 1.05 & $(0.90,1.22)$ & $1.16^{* *}$ & $(1.06,1.28)$ & $1.20 * *$ & $(1.08,1.34)$ & $1.15^{*}$ & $(1.02,1.29)$ \\
\hline Not MSA & 0.91 & $(0.74,1.12)$ & 1.11 & $(0.98,1.27)$ & $1.38 * * *$ & $(1.16,1.65)$ & $1.44 * * *$ & $(1.19,1.75)$ \\
\hline \multicolumn{9}{|l|}{ Education } \\
\hline Some high school or less (ref) & 1.0 & & 1.0 & & 1.0 & & 1.0 & \\
\hline High school diploma or GED & $1.80 * * *$ & $(1.48,2.19)$ & 1.10 & $(0.91,1.32)$ & 1.09 & $(0.92,1.30)$ & 1.08 & $(0.92,1.28)$ \\
\hline \multicolumn{9}{|l|}{ Insurance } \\
\hline Private or Medi-Gap (ref) & 1.0 & & 1.0 & & 1.0 & & 1.0 & \\
\hline Medicaid, CHIP, State-sponsored & $0.52 * * *$ & $(0.42,0.64)$ & 1.06 & $(0.89,1.27)$ & 1.07 & $(0.91,1.26)$ & 1.01 & $(0.84,1.20)$ \\
\hline Medicare, military, government & $0.40 * * *$ & $(0.30,0.53)$ & 1.06 & $(0.87,1.30)$ & 0.84 & $(0.67,1.06)$ & $1.49 * *$ & $(1.13,1.97)$ \\
\hline Single-service, Indian Health Service, not covered & $0.63 * * *$ & $(0.50,0.78)$ & 0.88 & $(0.77,1.00)$ & 0.90 & $(0.79,1.04)$ & $0.25 * * *$ & $(0.22,0.29)$ \\
\hline \multicolumn{9}{|l|}{ Household income, \% of federal poverty level } \\
\hline $0-99$ (ref) & 1.0 & & 1.0 & & 1.0 & & 1.0 & \\
\hline $100-299$ & $1.49 * * *$ & $(1.25,1.76)$ & 1.06 & $(0.91,1.24)$ & 1.00 & $(0.88,1.14)$ & 1.13 & $(0.99,1.30)$ \\
\hline$>\mathbf{3 0 0} \%$ & $2.25 * * *$ & $(1.77,2.85)$ & 1.08 & $(0.91,1.28)$ & $0.78 * *$ & $(0.67,0.90)$ & $1.32 * *$ & $(1.12,1.58)$ \\
\hline \multicolumn{9}{|l|}{ Access to Usual Source of Care } \\
\hline Yes (ref) & 1.0 & & 1.0 & & 1.0 & & & \\
\hline No & $0.68 * * *$ & $(0.56,0.81)$ & 0.90 & $(0.79,1.01)$ & $0.77 * * *$ & $(0.67,0.88)$ & & \\
\hline \multicolumn{9}{|l|}{ BMI } \\
\hline 18.5-24.9 (Normal) (ref) & 1.0 & & & & & & 1.0 & \\
\hline$<18.5$ (Underweight) & 0.71 & $(0.37,1.33)$ & & & & & 0.91 & $(0.61,1.36)$ \\
\hline 25.0-29.9 (Overweight) & 1.03 & $(0.85,1.25)$ & & & & & 1.09 & $(0.96,1.24)$ \\
\hline$>\mathbf{3 0}($ Obese $)$ & $0.52 * * *$ & $(0.44,0.62)$ & & & & & $1.27 * * *$ & $(1.11,1.45)$ \\
\hline
\end{tabular}

Note. Sample does not include pregnant females or those missing values listwise on these variables.

Self-rated general health $=0$ ) Poor, Fair 1) Good, Very good, Excellent. Logistic regression

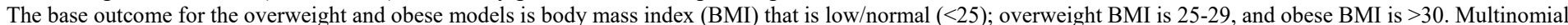
regression.

Access to a usual source of care $=0$ ) No 1) Yes. Logistic regression.

$\mathrm{OR}=$ odds ratio; $\mathrm{RRR}=$ relative risk ratio; $\mathrm{CI}=$ confidence interval; $\mathrm{MSA}=$ metropolitan statistical area.

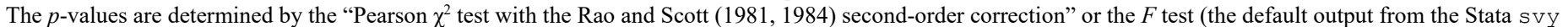

tabulate command); $* p<0.05, * * p<0.01, * * * p<0.001$ 
Table 1-F. Regressions of Health Outcomes on Sexual Attraction for Adults Age 25-45 (N=23,567)

\begin{tabular}{|c|c|c|c|c|c|c|c|c|}
\hline & \multicolumn{2}{|c|}{ Self-Rated Health } & \multicolumn{2}{|c|}{ Overweight } & \multicolumn{2}{|c|}{ Obese } & \multicolumn{2}{|c|}{ Usual Source of Care } \\
\hline & OR & $95 \%$ CI & RRR & $95 \% \mathrm{CI}$ & RRR & $95 \%$ CI & OR & $95 \%$ CI \\
\hline Intercept & $14.32 * * *$ & $(9.63,21.29)$ & $0.55^{* * *}$ & $(0.43,0.71)$ & $0.69 * *$ & $(0.54,0.88)$ & $4.41^{* * *}$ & $(3.39,5.75)$ \\
\hline \multicolumn{9}{|l|}{ Sexual Attraction } \\
\hline Only attracted to opposite sex (ref) & 1.0 & & 1.0 & & 1.0 & & 1.0 & \\
\hline Mostly attracted to opposite sex & 0.89 & $(0.69,1.13)$ & 0.85 & $(0.72,1.02)$ & 0.99 & $(0.83,1.18)$ & 0.89 & $(0.75,1.05)$ \\
\hline Equally attracted to males $\&$ females & $0.62 * *$ & $(0.47,0.82)$ & 0.87 & $(0.63,1.19)$ & 1.25 & $(0.95,1.65)$ & 0.91 & $(0.66,1.27)$ \\
\hline Mostly attracted to same sex & 0.72 & $(0.38,1.36)$ & 0.76 & $(0.44,1.30)$ & 0.88 & $(0.59,1.32)$ & 1.97 & $(0.88,4.44)$ \\
\hline Only attracted to same sex & 0.70 & $(0.41,1.22)$ & 0.75 & $(0.48,1.17)$ & 0.84 & $(0.58,1.21)$ & 1.27 & $(0.84,1.91)$ \\
\hline \multicolumn{9}{|l|}{ Survey Cycle } \\
\hline 2011-2013 (ref) & 1.0 & & 1.0 & & 1.0 & & & \\
\hline 2013-2015 & $0.77 * *$ & $(0.65,0.92)$ & $0.85^{*}$ & $(0.74,0.98)$ & 0.92 & $(0.81,1.06)$ & 0.97 & $(0.82,1.15)$ \\
\hline 2015-2017 & 0.85 & $(0.68,1.06)$ & 0.89 & $(0.77,1.02)$ & 0.97 & $(0.83,1.14)$ & 1.05 & $(0.89,1.25)$ \\
\hline 2017-2019 & $0.79 * *$ & $(0.66,0.94)$ & 1.01 & $(0.89,1.16)$ & 1.11 & $(0.94,1.31)$ & $0.83^{*}$ & $(0.69,0.99)$ \\
\hline \multicolumn{9}{|l|}{ Sex } \\
\hline Female (ref) & 1.0 & & 1.0 & & 1.0 & & 1.0 & \\
\hline Male & 1.05 & $(0.90,1.22)$ & $2.34 * * *$ & $(2.11,2.59)$ & $1.53^{* * *}$ & $(1.37,1.71)$ & $0.35^{* * *}$ & $(0.31,0.39)$ \\
\hline \multicolumn{9}{|l|}{ Age } \\
\hline 25-34 (ref) & 1.0 & & 1.0 & & 1.0 & & 1.0 & \\
\hline $35-44$ & $0.54 * * *$ & $(0.47,0.63)$ & $1.41^{* * *}$ & $(1.29,1.55)$ & $1.68 * * *$ & $(1.51,1.85)$ & $1.63^{* * *}$ & $(1.45,1.82)$ \\
\hline \multicolumn{9}{|l|}{ Race/ethnicity } \\
\hline White, non-Hispanic (ref) & 1.0 & & 1.0 & & 1.0 & & 1.0 & \\
\hline Hispanic & $0.81 *$ & $(0.66,1.00)$ & $1.48^{* * *}$ & $(1.28,1.71)$ & $1.84 * * *$ & $(1.59,2.14)$ & 0.95 & $(0.82,1.11)$ \\
\hline Black, non-Hispanic & 1.07 & $(0.85,1.34)$ & $1.31^{* * *}$ & $(1.13,1.51)$ & $2.16^{* * *}$ & $(1.89,2.48)$ & $1.23 *$ & $(1.05,1.45)$ \\
\hline Other, non-Hispanic & 0.84 & $(0.64,1.09)$ & $0.74 * *$ & $(0.63,0.88)$ & 0.88 & $(0.73,1.05)$ & $1.22 *$ & $(1.02,1.47)$ \\
\hline \multicolumn{9}{|l|}{ Nativity } \\
\hline US-born (ref) & 1.0 & & 1.0 & & 1.0 & & 1.0 & \\
\hline Foreign-born & $1.51 * * *$ & $(1.23,1.86)$ & $0.78 * *$ & $(0.67,0.90)$ & $0.44 * * *$ & $(0.38,0.51)$ & $0.79 * *$ & $(0.68,0.91)$ \\
\hline \multicolumn{9}{|l|}{ Residence } \\
\hline Principal MSA city (ref) & 1.0 & & 1.0 & & 1.0 & & 1.0 & \\
\hline Other MSA & 1.05 & $(0.90,1.21)$ & $1.16^{* *}$ & $(1.05,1.28)$ & $1.20^{* *}$ & $(1.08,1.34)$ & $1.15^{*}$ & $(1.02,1.29)$ \\
\hline Not MSA & 0.91 & $(0.74,1.12)$ & 1.10 & $(0.97,1.26)$ & $1.38 * * *$ & $(1.15,1.65)$ & $1.44^{* * *}$ & $(1.18,1.74)$ \\
\hline \multicolumn{9}{|l|}{ Education } \\
\hline Some high school or less (ref) & 1.0 & & 1.0 & & 1.0 & & 1.0 & \\
\hline High school diploma or GED & $1.81 * * *$ & $(1.49,2.20)$ & 1.10 & $(0.91,1.32)$ & 1.09 & $(0.92,1.30)$ & 1.08 & $(0.92,1.28)$ \\
\hline Some college & $1.95 * * *$ & $(1.53,2.50)$ & 1.08 & $(0.89,1.32)$ & 1.14 & $(0.94,1.38)$ & $1.37^{* *}$ & $(1.12,1.68)$ \\
\hline College or graduate degree & $3.13 * * *$ & $(2.46,3.98)$ & $0.83 *$ & $(0.69,1.00)$ & $0.68^{* * *}$ & $(0.57,0.82)$ & $1.44^{* * *}$ & $(1.18,1.75)$ \\
\hline \multicolumn{9}{|l|}{ Insurance } \\
\hline Private or Medi-Gap (ref) & 1.0 & & 1.0 & & 1.0 & & 1.0 & \\
\hline Medicaid, CHIP, State-sponsored & $0.52 * * *$ & $(0.42,0.64)$ & 1.07 & $(0.89,1.28)$ & 1.08 & $(0.92,1.26)$ & 1.01 & $(0.92,1.28)$ \\
\hline Medicare, military, government & $0.40 * * *$ & $(0.30,0.53)$ & 1.06 & $(0.87,1.30)$ & 0.84 & $(0.67,1.06)$ & $1.49^{* *}$ & $(1.12,1.96)$ \\
\hline Single-service, Indian Health Service, not covered & $0.63 * * *$ & $(0.50,0.78)$ & 0.88 & $(0.77,1.01)$ & 0.90 & $(0.79,1.04)$ & $0.25^{* * *}$ & $(0.22,0.29)$ \\
\hline \multicolumn{9}{|l|}{ Household income, \% of federal poverty level } \\
\hline $0-99($ ref) & 1.0 & & 1.0 & & 1.0 & & 1.0 & \\
\hline $100-299$ & $1.48 * * *$ & $(1.25,1.76)$ & 1.07 & $(0.91,1.24)$ & 1.00 & $(0.88,1.15)$ & 1.13 & $(0.99,1.30)$ \\
\hline$>\mathbf{3 0 0} \%$ & $2.25 * * *$ & $(1.77,2.85)$ & 1.08 & $(0.91,1.28)$ & $0.78 * *$ & $(0.67,0.90)$ & $1.33 * *$ & $(1.12,1.58)$ \\
\hline \multicolumn{9}{|l|}{ Access to Usual Source of Care } \\
\hline Yes (ref) & 1.0 & & 1.0 & & 1.0 & & & \\
\hline No & $0.68 * * *$ & $(0.57,0.81)$ & 0.90 & $(0.79,1.02)$ & $0.77 * * *$ & $(0.67,0.88)$ & & \\
\hline \multicolumn{9}{|l|}{ BMI } \\
\hline 18.5-24.9 (Normal) (ref) & 1.0 & & & & & & 1.0 & \\
\hline$<\mathbf{1 8 . 5}$ (Underweight) & 0.71 & $(0.37,1.33)$ & & & & & 0.91 & $(0.60,1.36)$ \\
\hline 25.0-29.9 (Overweight) & 1.03 & $(0.85,1.24)$ & & & & & 1.09 & $(0.96,1.24)$ \\
\hline$>30.0$ (Obese) & $0.52 * * *$ & $(0.44,0.62)$ & & & & & $1.27^{* * *}$ & $(1.11,1.45)$ \\
\hline
\end{tabular}

Note. Sample does not include pregnant females or those missing values listwise on these variables.

Self-rated general health $=0$ ) Poor, Fair 1) Good, Very good, Excellent. Logistic regression

The base outcome for the overweight and obese models is body mass index (BMI) that is low/normal ( $<25)$; overweight BMI is $25-29$, and obese BMI is $>30$. Multinomial regression.

Access to a usual source of care $=0$ ) No 1) Yes. Logistic regression.

$\mathrm{OR}=$ odds ratio $\mathrm{RRR}=$ relative risk ratio $\mathrm{CI}=$ confidence interval; $\mathrm{MSA}=$ metropolitan statistical area.

The $p$-values are determined by the "Pearson $\chi^{2}$ test with the Rao and Scott $(1981,1984)$ second-order correction" or the $F$ test (the default output from the Stata svy

tabulate command); ${ }^{*} p<0.05, * * p<0.01, * * * p<0.001$ 
Table 1-G. Regressions of Health Outcomes on Sexual Behavior for Adults Age 25-45 (N=23,567)

\begin{tabular}{|c|c|c|c|c|c|c|c|c|}
\hline & \multicolumn{2}{|c|}{ Self-Rated Health } & \multicolumn{2}{|c|}{ Overweight } & \multicolumn{2}{|c|}{ Obese } & \multicolumn{2}{|c|}{ Usual Source of Care } \\
\hline & OR & $95 \%$ CI & RRR & $95 \% \mathrm{CI}$ & RRR & $95 \% \mathrm{CI}$ & OR & $95 \%$ CI \\
\hline Intercept & $14.79 * * *$ & $(9.92,22.04)$ & $0.54^{* * *}$ & $(0.42,0.69)$ & $0.68 * *$ & $(0.53,0.87)$ & $4.24^{* * *}$ & $(3.24,5.56)$ \\
\hline \multicolumn{9}{|l|}{ Sexual Behavior } \\
\hline No sexual experience with same-sex partner (ref) & 1.0 & & 1.0 & & 1.0 & & 1.0 & \\
\hline Sexual experience with same-sex partner & $0.70 * * *$ & $(0.58,0.85)$ & 0.97 & $(0.82,1.13)$ & 1.05 & $(0.92,1.21)$ & 1.14 & $(0.97,1.32)$ \\
\hline \multicolumn{9}{|l|}{ Survey Cycle } \\
\hline 2011-2013 (ref) & 1.0 & & 1.0 & & 1.0 & & 1.0 & \\
\hline 2013-2015 & $0.77 * *$ & $(0.65,0.92)$ & $0.85^{*}$ & $(0.74,0.98)$ & 0.92 & $(0.81,1.05)$ & 0.97 & $(0.82,1.14)$ \\
\hline 2015-2017 & 0.85 & $(0.68,1.06)$ & 0.88 & $(0.77,1.02)$ & 0.97 & $(0.83,1.13)$ & 1.06 & $(0.89,1.25)$ \\
\hline 2017-2019 & $0.79 * *$ & $(0.66,0.94)$ & 1.01 & $(0.88,1.15)$ & 1.11 & $(0.94,1.31)$ & $0.82 *$ & $(0.69,0.98)$ \\
\hline \multicolumn{9}{|l|}{ Sex } \\
\hline Female (ref) & 1.0 & & 1.0 & & 1.0 & & 1.0 & \\
\hline Male & 1.02 & $(0.88,1.19)$ & $2.37 * * *$ & $(2.14,2.62)$ & $1.53 * * *$ & $(1.37,1.71)$ & $0.36 * * *$ & $(0.32,0.40)$ \\
\hline \multicolumn{9}{|l|}{ Age } \\
\hline 25-34 (ref) & 1.0 & & 1.0 & & 1.0 & & 1.0 & \\
\hline $35-44$ & $0.55 * * *$ & $(0.47,0.63)$ & $1.42 * * *$ & $(1.30,1.56)$ & $1.67 * * *$ & $(1.51,1.85)$ & $1.63^{* * *}$ & $(1.46,1.83)$ \\
\hline \multicolumn{9}{|l|}{ Race/ethnicity } \\
\hline White, non-Hispanic (ref) & 1.0 & & 1.0 & & 1.0 & & 1.0 & \\
\hline Hispanic & $0.80 *$ & $(0.65,0.99)$ & $1.48 * * *$ & $(1.28,1.71)$ & $1.84 * * *$ & $(1.58,2.14)$ & 0.96 & $(0.83,1.12)$ \\
\hline Black, non-Hispanic & 1.06 & $(0.84,1.32)$ & $1.31^{* * *}$ & $(1.13,1.52)$ & $2.16^{* * *}$ & $(1.89,2.48)$ & $1.25^{* *}$ & $(1.06,1.47)$ \\
\hline Other, non-Hispanic & 0.84 & $(0.64,1.09)$ & $0.74 * *$ & $(0.63,0.88)$ & 0.88 & $(0.73,1.05)$ & $1.23^{*}$ & $(1.02,1.48)$ \\
\hline \multicolumn{9}{|l|}{ Nativity } \\
\hline US-born (ref) & 1.0 & & 1.0 & & 1.0 & & 1.0 & \\
\hline Foreign-born & $1.48^{* * *}$ & $(1.21,1.82)$ & $0.78 * *$ & $(0.68,0.90)$ & $0.44 * * *$ & $(0.38,0.51)$ & $0.79 * *$ & $(0.69,0.92)$ \\
\hline \multicolumn{9}{|l|}{ Residence } \\
\hline Principal MSA city (ref) & 1.0 & & 1.0 & & 1.0 & & 1.0 & \\
\hline Other MSA & 1.04 & $(0.90,1.21)$ & $1.16^{* *}$ & $(1.06,1.28)$ & $1.21^{* *}$ & $(1.08,1.34)$ & $1.15^{*}$ & $(1.02,1.30)$ \\
\hline Not MSA & 0.90 & $(0.73,1.12)$ & 1.11 & $(0.97,1.27)$ & $1.38 * * *$ & $(1.16,1.65)$ & $1.45^{* * *}$ & $(1.19,1.75)$ \\
\hline \multicolumn{9}{|l|}{ Education } \\
\hline Some high school or less (ref) & 1.0 & & 1.0 & & 1.0 & & 1.0 & \\
\hline High school diploma or GED & $1.81^{* * *}$ & $(1.50,2.20)$ & 1.09 & $(0.91,1.32)$ & 1.09 & $(0.91,1.30)$ & 1.08 & $(0.92,1.28)$ \\
\hline Some college & $1.97 * * *$ & $(1.54,2.51)$ & 1.08 & $(0.89,1.31)$ & 1.13 & $(0.94,1.37)$ & $1.37^{* *}$ & $(1.12,1.67)$ \\
\hline College or graduate degree & $3.14 * * *$ & $(2.47,3.98)$ & $0.82 *$ & $(0.68,0.99)$ & $0.68^{* * *}$ & $(0.57,0.82)$ & $1.43 * * *$ & $(1.18,1.74)$ \\
\hline \multicolumn{9}{|l|}{ Insurance } \\
\hline Private or Medi-Gap (ref) & 1.0 & & 1.0 & & 1.0 & & 1.0 & \\
\hline Medicaid, CHIP, State-sponsored & $0.52 * * *$ & $(0.42,0.65)$ & 1.06 & $(0.89,1.27)$ & 1.08 & $(0.92,1.26)$ & 1.00 & $(0.84,1.20)$ \\
\hline Medicare, military, government & $0.40 * * *$ & $(0.30,0.53)$ & 1.06 & $(0.87,1.30)$ & 0.84 & $(0.67,1.06)$ & $1.48^{* *}$ & $(1.12,1.96)$ \\
\hline Single-service, Indian Health Service, not covered & $0.63 * * *$ & $(0.51,0.79)$ & 0.88 & $(0.77,1.01)$ & 0.90 & $(0.79,1.04)$ & $0.25^{* * *}$ & $(0.22,0.29)$ \\
\hline \multicolumn{9}{|l|}{ Household income, \% of federal poverty level } \\
\hline $0-99$ (ref) & 1.0 & & 1.0 & & 1.0 & & 1.0 & \\
\hline $100-299$ & $1.48^{* * *}$ & $(1.25,1.76)$ & 1.07 & $(0.91,1.24)$ & 1.00 & $(0.88,1.15)$ & 1.13 & $(0.99,1.30)$ \\
\hline$>\mathbf{3 0 0} \%$ & $2.23 * * *$ & $(1.76,2.83)$ & 1.08 & $(0.91,1.28)$ & $0.78 * *$ & $(0.67,0.90)$ & $1.33 * *$ & $(1.12,1.58)$ \\
\hline \multicolumn{9}{|l|}{ Access to Usual Source of Care } \\
\hline Yes (ref) & 1.0 & & 1.0 & & 1.0 & & & \\
\hline No & $0.68 * * *$ & $(0.57,0.81)$ & 0.90 & $(0.79,1.02)$ & $0.77^{* * *}$ & $(0.67,0.88)$ & & \\
\hline \multicolumn{9}{|l|}{ BMI } \\
\hline 18.5-24.9 (Normal) (ref) & 1.0 & & & & & & 1.0 & \\
\hline$<18.5$ (Underweight) & 0.70 & $(0.37,1.32)$ & & & & & 0.91 & $(0.61,1.37)$ \\
\hline 25.0-29.9 (Overweight) & 1.03 & $(0.85,1.25)$ & & & & & 1.09 & $(0.96,1.24)$ \\
\hline$>30.0$ (Obese) & $0.52 * * *$ & $(0.44,0.62)$ & & & & & $1.27 * * *$ & $(1.11,1.44)$ \\
\hline
\end{tabular}

Note. Sample does not include pregnant females or those missing values listwise on these variables.

Self-rated general health $=0$ ) Poor, Fair 1) Good, Very good, Excellent. Logistic regression

The base outcome for the overweight and obese models is body mass index (BMI) that is low/normal ( $<25)$; overweight BMI is $25-29$, and obese BMI is $>30$. Multinomial regression.

Access to a usual source of care $=0$ ) No 1) Yes. Logistic regression.

$\mathrm{OR}=$ odds ratio $; \mathrm{RRR}=$ relative risk ratio $\mathrm{CI}=$ confidence interval $; \mathrm{MSA}=$ metropolitan statistical area.

The $p$-values are determined by the "Pearson $\chi^{2}$ test with the Rao and Scott $(1981,1984)$ second-order correction" or the $F$ test (the default output from the Stata svy tabulate command); ${ }^{*} p<0.05, * * p<0.01, * * * p<0.001$ 
TABLE 2-A. CH. 2 SUMMARY STATISTICS: IDENTITY: FEMALE

Table 2-A. Characteristics of US Females Aged 25-45 Years, by Sexual Identity 2011-2019 (N=12,895)

\begin{tabular}{|c|c|c|c|c|c|c|}
\hline & \multicolumn{6}{|c|}{ Sexual Identity } \\
\hline & \multicolumn{2}{|c|}{$\begin{array}{l}\text { Heterosexual or straight } \\
\qquad(\mathrm{n}=11,790 ; 91.4 \%)^{\mathrm{a}}\end{array}$} & \multicolumn{2}{|c|}{$\begin{array}{c}\text { Homosexual, gay, or } \\
\text { lesbian } \\
(\mathrm{n}=282 ; 2.2 \%)^{\mathrm{a}}\end{array}$} & \multicolumn{2}{|c|}{$\begin{array}{c}\text { Bisexual } \\
(\mathrm{n}=823 ; 6.4 \%)^{\mathrm{a}}\end{array}$} \\
\hline & Mean \% & $95 \%$ CI & Mean \% & $95 \% \mathrm{CI}$ & Mean \% & $95 \%$ CI \\
\hline \multicolumn{7}{|l|}{ Sexual Attraction*** } \\
\hline Only attracted to males & 87.4 & $(86.3,88.4)$ & 3.1 & $(1.1,8.4)$ & 3.3 & $(2.1,5.1)$ \\
\hline Mostly attracted to males & 11.8 & $(10.8,12.9)$ & 1.1 & $(0.4,2.7)$ & 42.0 & $(37.0,47.1)$ \\
\hline Equally attracted to males \& females & 0.7 & $(0.5,0.9)$ & 2.7 & $(1.4,5.3)$ & 49.4 & $(44.5,54.4)$ \\
\hline Mostly attracted to females & 0.0 & $(0.0,0.0)$ & 29.1 & $(22.3,36.9)$ & 5.1 & $(3.2,8.0)$ \\
\hline Only attracted to females & 0.1 & $(0.0,0.1)$ & 64.0 & $(56.2,71.2)$ & 0.2 & $(0.1,1.2)$ \\
\hline \multicolumn{7}{|l|}{ Sexual Behavior $* * * *$} \\
\hline Sexual experience with same-sex partner & 12.7 & $(11.7,13.7)$ & 94.9 & $(89.5,97.6)$ & 83.1 & $(78.5,87.0)$ \\
\hline No sexual experience with same-sex partner & 87.3 & $(86.3,88.3)$ & 5.1 & $(2.4,10.5)$ & 16.9 & $(13.0,21.5)$ \\
\hline \multicolumn{7}{|l|}{$\operatorname{Age}^{* * *}$} \\
\hline $25-34$ & 49.4 & $(48.1,50.7)$ & 64.0 & $(55.5,71.7)$ & 65.7 & $(60.9,70.1)$ \\
\hline $35-44$ & 50.6 & $(49.3,51.9)$ & 36.0 & $(28.3,44.5)$ & 34.3 & $(29.9,39.1)$ \\
\hline \multicolumn{7}{|l|}{ Race/ethnicity* } \\
\hline White, non-Hispanic & 57.3 & $(55.0,59.6)$ & 57.8 & $(48.9,66.2)$ & 63.5 & $(58.0,68.6)$ \\
\hline Hispanic & 19.5 & $(17.5,21.6)$ & 14.1 & $(9.8,19.9)$ & 13.0 & $(9.7,17.2)$ \\
\hline Black, non-Hispanic & 13.1 & $(11.7,14.7)$ & 16.1 & $(10.9,23.2)$ & 13.9 & $(11.0,17.3)$ \\
\hline Other, non-Hispanic & 10.1 & $(8.9,11.4)$ & 11.9 & $(7.5,18.6)$ & 9.7 & $(6.8,13.5)$ \\
\hline \multicolumn{7}{|l|}{ Residence } \\
\hline Principal MSA city & 32.9 & $(30.1,35.8)$ & 40.5 & $(31.3,50.4)$ & 37.3 & $(32.0,43.0)$ \\
\hline Other MSA & 50.4 & $(47.2,53.5)$ & 45.4 & $(36.2,54.9)$ & 48.3 & $(42.7,54.0)$ \\
\hline Not MSA & 16.7 & $(13.7,20.2)$ & 14.1 & (\#.\#,\#.\#) & 14.3 & $(10.0,20.2)$ \\
\hline \multicolumn{7}{|l|}{ Nativity $* * *$} \\
\hline US-born & 82.1 & $(80.5,83.6)$ & 88.9 & $(80.4,93.9)$ & 93.8 & $(91.1,95.7)$ \\
\hline Foreign-born & 17.9 & $(16.4,19.5)$ & 11.1 & $(6.1,19.6)$ & 6.2 & $(4.3,8.9)$ \\
\hline \multicolumn{7}{|l|}{ Education } \\
\hline Some high school or less & 9.4 & $(8.4,10.5)$ & 7.0 & $(3.9,12.4)$ & 8.4 & $(6.3,11.2)$ \\
\hline High school diploma or GED & 22.6 & $(21.2,23.9)$ & 28.2 & $(20.9,36.9)$ & 25.3 & $(20.8,30.4)$ \\
\hline Some college & 19.5 & $(18.3,20.7)$ & 23.2 & $(17.0,30.8)$ & 23.6 & $(19.8,28.0)$ \\
\hline College or graduate degree & 48.5 & $(46.4,50.7)$ & 41.6 & $(33.6,50.0)$ & 42.6 & $(36.9,48.6)$ \\
\hline \multicolumn{7}{|l|}{ Insurance $* * *$} \\
\hline Private or Medi-Gap & 64.2 & $(62.3,66.0)$ & 61.1 & $(52.2,69.3)$ & 51.0 & $(46.0,55.9)$ \\
\hline Medicaid, CHIP, State-sponsored & 15.5 & $(14.2,16.8)$ & 12.5 & $(8.9,17.2)$ & 24.5 & $(20.7,28.8)$ \\
\hline Medicare, military, government & 4.4 & $(3.6,5.3)$ & 6.1 & $(3.4,10.7)$ & 4.9 & $(3.2,7.3)$ \\
\hline Single-service, Indian Health Service, not covered & 16.0 & $(14.7,17.3)$ & 20.3 & $(13.4,29.6)$ & 19.6 & $(15.8,24.2)$ \\
\hline \multicolumn{7}{|l|}{ Household income, $\%$ of federal poverty level $* *$} \\
\hline $0-99$ & 21.7 & $(20.4,23.1)$ & 27.8 & $(20.3,36.8)$ & 24.1 & $(20.7,28.0)$ \\
\hline 100-299 & 37.2 & $(35.6,38.8)$ & 32.7 & $(24.9,41.6)$ & 43.8 & $(39.3,48.4)$ \\
\hline$>300 \%$ & 41.1 & $(39.1,43.2)$ & 39.5 & $(30.3,49.6)$ & 32.1 & $(27.2,37.4)$ \\
\hline \multicolumn{7}{|l|}{ Self-rated health $* * *$} \\
\hline Poor & 1.2 & $(1.0,1.6)$ & 5.7 & $(1.9,15.5)$ & 2.1 & $(1.4,3.3)$ \\
\hline Fair & 7.2 & $(6.6,8.0)$ & 7.6 & $(5.0,11.3)$ & 11.2 & $(8.8,14.3)$ \\
\hline Good & 25.6 & $(24.4,26.8)$ & 30.4 & $(23.8,37.9)$ & 34.0 & $(29.0,39.5)$ \\
\hline Very good & 38.6 & $(37.2,40.0)$ & 27.1 & $(20.3,35.2)$ & 32.1 & $(27.4,37.3)$ \\
\hline Excellent & 27.4 & $(26.0,28.7)$ & 29.3 & $(20.9,39.4)$ & 20.5 & $(16.7,24.9)$ \\
\hline \multicolumn{7}{|l|}{ BMI } \\
\hline <18.5 (Underweight) & 1.5 & $(1.2,1.8)$ & 0.9 & $(0.3,3.0)$ & 0.8 & $(0.4,1.7)$ \\
\hline 18.5-24.9 (Normal) & 39.2 & $(37.7,40.8)$ & 37.7 & $(29.1,47.1)$ & 34.9 & $(30.1,40.0)$ \\
\hline 25.0-29.9 (Overweight) & 25.8 & $(24.7,27.0)$ & 27.9 & $(20.8,36.3)$ & 27.0 & $(22.9,31.6)$ \\
\hline$>30.0$ (Obese) & 33.5 & $(32.0,35.0)$ & 33.5 & $(26.1,41.8)$ & 37.2 & $(32.4,42.3)$ \\
\hline Access to Usual Source of Care* & & & & & & \\
\hline Yes & 87.5 & $(86.5,88.5)$ & 82.7 & $(74.0,88.9)$ & 82.8 & $(78.7,86.2)$ \\
\hline No & 12.5 & $(11.5,13.5)$ & 17.3 & $(11.1,26.0)$ & 17.2 & $(13.8,21.3)$ \\
\hline Cycle $* * *$ & & & & & & \\
\hline 2011-2013 & 25.4 & $(23.3,27.6)$ & 15.2 & $(9.6,23.2)$ & 20.7 & $(16.6,25.5)$ \\
\hline 2013-2015 & 26.4 & $(24.3,28.6)$ & 26.5 & $(19.8,34.4)$ & 22.4 & $(18.1,27.4)$ \\
\hline 2015-2017 & 24.5 & $(22.3,27.0)$ & 32.9 & $(22.8,44.9)$ & 23.6 & $(19.6,28.2)$ \\
\hline 2017-2019 & 23.7 & $(21.5,26.0)$ & 25.4 & $(19.1,32.9)$ & 33.2 & $(28.1,38.9)$ \\
\hline
\end{tabular}

${ }^{a} \mathrm{n}=$ sample number and percent without using svy command; Mean and $95 \%$ CI were conducted using svy command.

${ }^{*} \mathrm{p}<0.05,{ }^{* *} \mathrm{p}<0.01,{ }^{* * *} \mathrm{p}<0.001 ; p$-values were determined using the "Pearson $\chi^{2}$ test with the Rao and Scott $(1981,1984)$ second-order correction" or the $F$ test (the default output from the Stata svy tabulate command) (Stata.com n.d.:11).

Note. Sample does not include pregnant females or those missing values listwise on these variables. MSA = metropolitan statistical area; $\mathrm{BMI}=$ body mass index; not all strata included in each cell if missing subpopulation members. 
TABLE 2-B. CH. 2 SUMMARY STATISTICS: ATTRACTION: FEMALE

Table 2-B. Characteristics of US Females Aged 25-45 Years, by Sexual Attraction 2011-2019 (N=12,895)

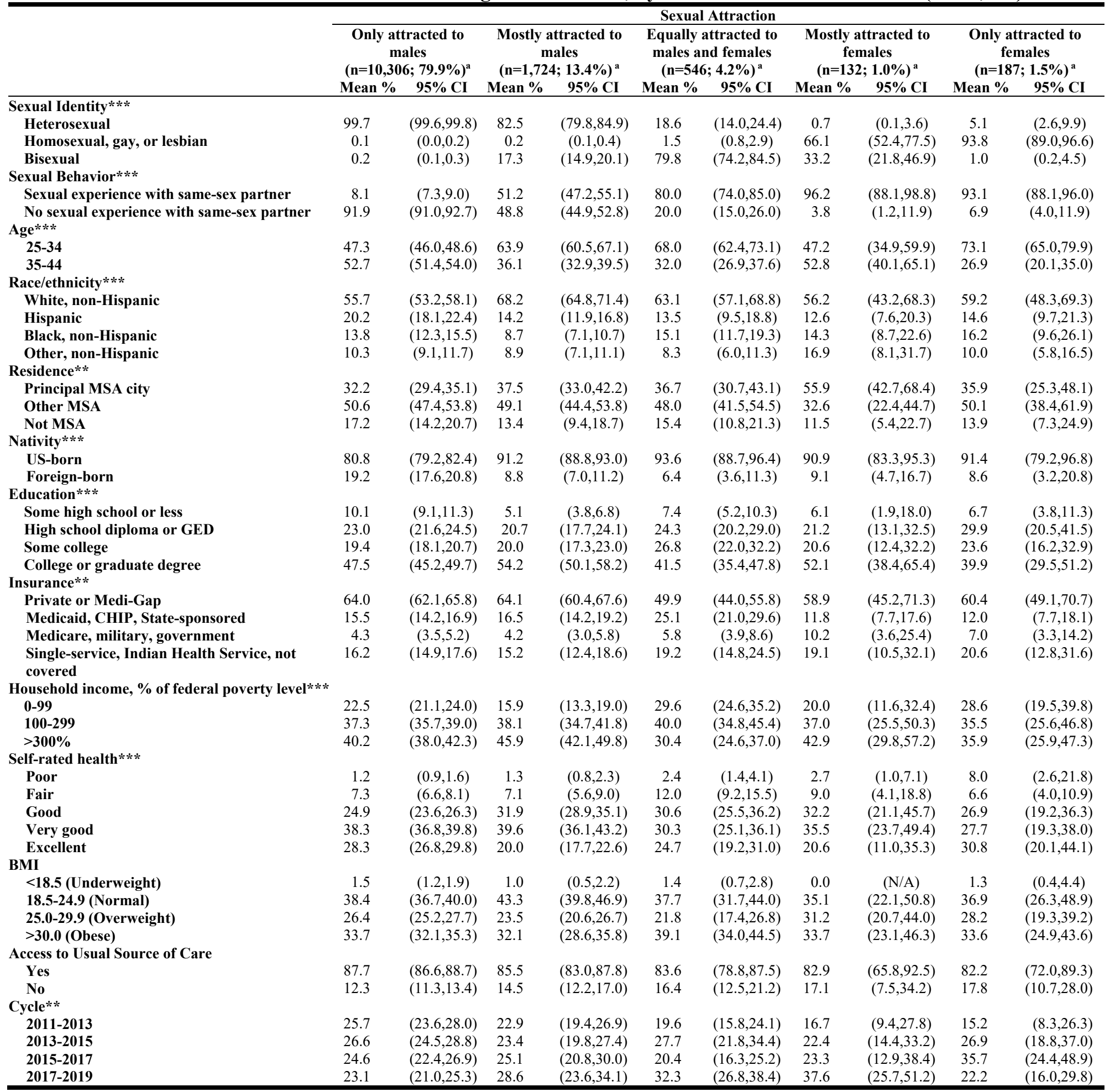

${ }^{a} \mathrm{n}=$ sample number and percent without using svy command; Mean and $95 \%$ CI were conducted using svy command.

${ }^{*} \mathrm{p}<0.05, * * \mathrm{p}<0.01,{ }^{* * *} \mathrm{p}<0.001 ; p$-values were determined using the "Pearson $\chi 2$ test with the Rao and Scott $(1981,1984)$ second-order correction" or the $F$ test (the default output from the Stata svy tabulate command) (Stata.com n.d.:11).

Note. Sample does not include pregnant females or those missing values listwise on these variables. $\mathrm{MSA}=$ metropolitan statistical area; $\mathrm{N} / \mathrm{A}=$ not applicable; $\mathrm{BMI}=$ body mass index; not all strata included in each cell if missing subpopulation members. 
Table 2-C. Characteristics of US Females Aged 25-45 Years, by Sexual Behavior 2011-2019 $(\mathrm{N}=12,895)$

\begin{tabular}{|c|c|c|c|c|}
\hline & \multicolumn{4}{|c|}{ Sexual Behavior } \\
\hline & \multicolumn{2}{|c|}{$\begin{array}{l}\text { Sexual experience } \\
\text { with same sex } \\
\text { partner } \\
(\mathrm{n}=\mathbf{2 , 5 5 0} ; \mathbf{1 9 . 8 \%} \%)^{\mathrm{a}}\end{array}$} & \multicolumn{2}{|c|}{$\begin{array}{c}\text { No sexual experience } \\
\text { with same sex } \\
\text { partner } \\
(\mathrm{n}=10,345 ; 80.2 \%)^{\mathrm{a}}\end{array}$} \\
\hline & Mean \% & $95 \%$ CI & Mean \% & $95 \%$ CI \\
\hline \multicolumn{5}{|l|}{ Sexual Identity**** } \\
\hline Heterosexual & 64.8 & $(61.7,67.7)$ & 98.8 & $(98.4,99.0)$ \\
\hline Homosexual, gay, or lesbian & 10.0 & $(8.3,12.0)$ & 0.1 & $(0.1,0.3)$ \\
\hline Bisexual & 25.2 & $(22.8,27.7)$ & 1.1 & $(0.9,1.5)$ \\
\hline \multicolumn{5}{|l|}{ Sexual Attraction $* * *$} \\
\hline Only attracted to males & 36.3 & $(33.4,39.4)$ & 91.1 & $(90.0,92.1)$ \\
\hline Mostly attracted to males & 37.5 & $(34.8,40.3)$ & 7.9 & $(7.0,9.0)$ \\
\hline Equally attracted to males \& females & 15.0 & $(13.1,17.1)$ & 0.8 & $(0.6,1.1)$ \\
\hline Mostly attracted to females & 4.5 & $(3.5,5.7)$ & 0.0 & $(0.0,0.1)$ \\
\hline Only attracted to females & 6.7 & $(5.4,8.4)$ & 0.1 & $(0.1,0.2)$ \\
\hline \multicolumn{5}{|l|}{ Age $* * *$} \\
\hline 25-34 & 57.9 & $(54.9,60.9)$ & 48.9 & $(47.6,50.2)$ \\
\hline $35-44$ & 42.1 & $(39.1,45.1)$ & 51.1 & $(49.8,52.4)$ \\
\hline \multicolumn{5}{|l|}{ Race/ethnicity*** } \\
\hline White, non-Hispanic & 64.8 & $(61.8,67.6)$ & 56.1 & $(53.6,58.5)$ \\
\hline Hispanic & 13.1 & $(11.2,15.3)$ & 20.3 & $(18.3,22.5)$ \\
\hline Black, non-Hispanic & 14.2 & $(12.1,16.6)$ & 13.0 & $(11.6,14.6)$ \\
\hline Other, non-Hispanic & 7.9 & $(6.5,9.7)$ & 10.6 & $(9.3,12.0)$ \\
\hline \multicolumn{5}{|l|}{ Residence* } \\
\hline Principal MSA city & 36.8 & $(32.9,40.8)$ & 32.5 & $(29.7,35.4)$ \\
\hline Other MSA & 46.4 & $(42.3,50.6)$ & 51.0 & $(47.8,54.1)$ \\
\hline Not MSA & 16.8 & $(12.6,21.9)$ & 16.5 & $(13.6,19.9)$ \\
\hline \multicolumn{5}{|l|}{ Nativity**: } \\
\hline US-born & 94.3 & $(92.7,95.5)$ & 80.3 & $(78.6,82.0)$ \\
\hline Foreign-born & 5.7 & $(4.5,7.3)$ & 19.7 & $(18.0,21.4)$ \\
\hline \multicolumn{5}{|l|}{ Education $* * *$} \\
\hline Some high school or less & 6.5 & $(5.4,7.9)$ & 9.9 & $(8.8,11.1)$ \\
\hline High school diploma or GED & 24.8 & $(22.0,27.8)$ & 22.4 & $(21.0,23.8)$ \\
\hline Some college & 25.0 & $(22.7,27.6)$ & 18.6 & $(17.4,19.9)$ \\
\hline College or graduate degree & 43.6 & $(40.0,47.3)$ & 49.1 & $(46.9,51.3)$ \\
\hline \multicolumn{5}{|l|}{ Insurance**** } \\
\hline Private or Medi-Gap & 56.4 & $(53.1,59.6)$ & 64.9 & $(63.0,66.9)$ \\
\hline Medicaid, CHIP, State-sponsored & 20.5 & $(18.1,23.2)$ & 14.9 & $(13.6,16.3)$ \\
\hline Medicare, military, government & 5.0 & $(4.0,6.4)$ & 4.3 & $(3.5,5.2)$ \\
\hline Single-service, Indian Health Service, not covered & 18.0 & $(15.5,20.8)$ & 15.9 & $(14.5,17.4)$ \\
\hline \multicolumn{5}{|l|}{ Household income, \% of federal poverty level* } \\
\hline $0-99$ & 23.6 & $(21.2,26.1)$ & 21.6 & $(20.2,23.1)$ \\
\hline 100-299 & 39.4 & $(36.6,42.3)$ & 37.1 & $(35.4,38.8)$ \\
\hline$>\mathbf{3 0 0} \%$ & 37.0 & $(34.0,40.2)$ & 41.3 & $(39.2,43.6)$ \\
\hline \multicolumn{5}{|l|}{ Self-rated health $* * *$} \\
\hline Poor & 2.1 & $(1.5,3.1)$ & 1.2 & $(0.9,1.6)$ \\
\hline Fair & 9.2 & $(7.8,10.9)$ & 7.1 & $(6.4,7.8)$ \\
\hline Good & 32.3 & $(29.6,35.0)$ & 24.8 & $(23.5,26.1)$ \\
\hline Very good & 34.7 & $(32.0,37.5)$ & 38.7 & $(37.3,40.2)$ \\
\hline Excellent & 21.7 & $(19.2,24.4)$ & 28.2 & $(26.7,29.7)$ \\
\hline \multicolumn{5}{|l|}{ BMI } \\
\hline$<18.5$ (Underweight) & 1.1 & $(0.7,1.7)$ & 1.5 & $(1.2,1.9)$ \\
\hline 18.5-24.9 (Normal) & 37.4 & $(34.2,40.7)$ & 39.3 & $(37.7,40.9)$ \\
\hline 25.0-29.9 (Overweight) & 25.9 & $(23.2,28.7)$ & 26.0 & $(24.7,27.2)$ \\
\hline$>30.0$ (Obese) & 35.7 & $(32.8,38.7)$ & 33.2 & $(31.6,34.9)$ \\
\hline \multicolumn{5}{|l|}{ Access to Usual Source of Care } \\
\hline Yes & 86.4 & $(84.3,88.2)$ & 87.3 & $(86.2,88.4)$ \\
\hline No & 13.6 & $(11.8,15.7)$ & 12.7 & $(11.6,13.8)$ \\
\hline \multicolumn{5}{|l|}{ Cycle* } \\
\hline 2011-2013 & 22.3 & $(19.6,25.3)$ & 25.5 & $(23.3,27.8)$ \\
\hline 2013-2015 & 25.5 & $(22.5,28.8)$ & 26.3 & $(24.2,28.6)$ \\
\hline 2015-2017 & 24.5 & $(21.3,27.9)$ & 24.7 & $(22.3,27.3)$ \\
\hline 2017-2019 & 27.7 & $(24.0,31.6)$ & 23.5 & $(21.2,25.9)$ \\
\hline
\end{tabular}

${ }^{a} \mathrm{n}=$ sample number and percent without using svy command; Mean and $95 \%$ CI were conducted using svy command.

${ }^{*} \mathrm{p}<0.05,{ }^{*} \mathrm{p}<0.01,{ }^{* * *} \mathrm{p}<0.001 ; p$-values were determined using the "Pearson $\chi 2$ test with the Rao and Scott (1981, 1984) second-order correction" or the $F$ test (the default output from the Stata svy tabulate command)

(Stata.com n.d.:11).

Note. Sample does not include pregnant females or those missing values listwise on these variables. MSA = metropolitan statistical area; BMI = body mass index; not all strata included in each cell if missing subpopulation members. 
TABLE 2-D. CH. 2 SUMMARY STATISTICS: COMPONENTS AND OUTCOMES: FEMALE

Table 2-D. Sample Characteristics of US Females by Sexual Identity Components and Health Outcomes 2011-2019 (N=12,895)

\begin{tabular}{|c|c|c|c|c|c|c|c|c|}
\hline & \multirow[b]{2}{*}{$\begin{array}{l}\text { Number in } \\
\text { thousands }\end{array}$} & \multirow[b]{2}{*}{$\begin{array}{l}\% \text { of Total } \\
\text { Sample }\end{array}$} & \multicolumn{5}{|c|}{ Self-Rated Health } & \multirow[b]{2}{*}{$\begin{array}{c}\text { *Outcome sig. } \\
\text { differences }\end{array}$} \\
\hline & & & Poor & Fair & Good & Very Good & Excellent & \\
\hline Total Adult Sample & 12,895 & 100.0 & $1.6 \%$ & $8.6 \%$ & $27.3 \%$ & $36.8 \%$ & $25.8 \%$ & \\
\hline \multicolumn{9}{|l|}{ Sexual Identity } \\
\hline Heterosexual $^{\mathrm{A}}$ & 11,790 & 91.4 & $1.5 \%$ & $8.2 \%$ & $26.7 \%$ & $37.4 \%$ & $26.2 \%$ & $\mathrm{~B}, \mathrm{C}$ \\
\hline Homosexual, gay, or lesbian' & 282 & 2.2 & $3.9 \%$ & $11.4 \%$ & $32.3 \%$ & $29.4 \%$ & $23.1 \%$ & A \\
\hline Bisexual $^{\mathrm{C}}$ & 823 & 6.4 & $3.3 \%$ & $12.9 \%$ & $33.5 \%$ & $30.1 \%$ & $20.2 \%$ & A \\
\hline \multicolumn{9}{|l|}{ Sexual Behavior } \\
\hline No sexual experience with same-sex partner ${ }^{A}$ & 10,345 & 80.2 & $1.4 \%$ & $8.0 \%$ & $26.0 \%$ & $37.4 \%$ & $27.2 \%$ & $\mathrm{~B}$ \\
\hline Sexual experience with same-sex partner ${ }^{B}$ & 2,550 & 19.8 & $2.7 \%$ & $11.0 \%$ & $32.4 \%$ & $34.1 \%$ & $19.8 \%$ & A \\
\hline \multicolumn{9}{|l|}{ Sexual Attraction } \\
\hline Only attracted to opposite $\operatorname{sex}^{\mathrm{A}}$ & 10,306 & 79.9 & $1.5 \%$ & $8.2 \%$ & $25.9 \%$ & $37.2 \%$ & $27.2 \%$ & $\mathrm{C}, \mathrm{E}$ \\
\hline Mostly attracted to opposite $\operatorname{sex}^{B}$ & 1,724 & 13.4 & $1.6 \%$ & $8.8 \%$ & $31.8 \%$ & $37.9 \%$ & $19.9 \%$ & $\mathrm{C}, \mathrm{E}$ \\
\hline Equally attracted to males $\&$ females ${ }^{\mathrm{C}}$ & 546 & 4.2 & $2.9 \%$ & $13.9 \%$ & $35.5 \%$ & $28.4 \%$ & $19.2 \%$ & $\mathrm{~A}, \mathrm{~B}$ \\
\hline Mostly attracted to same $\operatorname{sex}^{D}$ & 132 & 1.0 & $4.6 \%$ & $9.1 \%$ & $31.8 \%$ & $34.1 \%$ & $20.5 \%$ & \\
\hline Only attracted to same $\operatorname{sex}^{\mathrm{E}}$ & 187 & 1.5 & $4.8 \%$ & $10.7 \%$ & $30.5 \%$ & $31.0 \%$ & $23.0 \%$ & $\mathrm{~A}, \mathrm{~B}$ \\
\hline \multicolumn{9}{|l|}{ Age Group } \\
\hline $25-34^{\mathrm{A}}$ & 7,116 & 55.2 & $1.1 \%$ & $7.5 \%$ & $26.9 \%$ & $36.9 \%$ & $27.6 \%$ & $\mathrm{~B}$ \\
\hline \multirow[t]{3}{*}{$35-44^{B}$} & 5,779 & 44.8 & $2.3 \%$ & $9.9 \%$ & $27.7 \%$ & $36.7 \%$ & $23.5 \%$ & A \\
\hline & & \multicolumn{7}{|c|}{ Body Mass Index (BMI) } \\
\hline & $\begin{array}{l}\text { Number in } \\
\text { thousands }\end{array}$ & $\begin{array}{c}\% \text { of Total } \\
\text { Sample }\end{array}$ & $\begin{array}{c}<18.5 \\
\text { (Underweight) }\end{array}$ & $\begin{array}{c}20-24 \\
\text { (Normal) }\end{array}$ & $\begin{array}{c}25.0-29.9 \\
\text { (Overweight) }\end{array}$ & $\begin{array}{c}30-50 \\
\text { (Obese) }\end{array}$ & & $\begin{array}{c}\text { *Outcome sig. } \\
\text { differences }\end{array}$ \\
\hline Total Adult Sample & 12,895 & 100.0 & $1.4 \%$ & $35.2 \%$ & $25.7 \%$ & $37.7 \%$ & & \\
\hline \multicolumn{9}{|l|}{ Sexual Identity } \\
\hline Heterosexual $^{\mathrm{A}}$ & 11,790 & 91.4 & $1.4 \%$ & $35.6 \%$ & $25.7 \%$ & $37.4 \%$ & & $\mathrm{C}$ \\
\hline Homosexual, gay, or lesbian ${ }^{B}$ & 282 & 2.2 & $1.1 \%$ & $31.6 \%$ & $26.6 \%$ & $40.8 \%$ & & \\
\hline Bisexual $^{\mathrm{C}}$ & 823 & 6.4 & $1.2 \%$ & $31.0 \%$ & $26.1 \%$ & $41.7 \%$ & & A \\
\hline \multicolumn{9}{|l|}{ Sexual Behavior } \\
\hline No sexual experience with same-sex partner ${ }^{A}$ & 10,345 & 80.2 & $1.4 \%$ & $35.6 \%$ & $25.7 \%$ & $37.3 \%$ & & \\
\hline Sexual experience with same-sex partner ${ }^{B}$ & 2,550 & 19.8 & $1.3 \%$ & $33.5 \%$ & $25.8 \%$ & $39.4 \%$ & & \\
\hline \multicolumn{9}{|l|}{ Sexual Attraction } \\
\hline Only attracted to opposite $\operatorname{sex}^{\mathrm{A}}$ & 10,306 & 79.9 & $1.4 \%$ & $35.1 \%$ & $26.1 \%$ & $37.4 \%$ & & $\mathrm{C}$ \\
\hline Mostly attracted to opposite $\operatorname{sex}^{B}$ & 1,724 & 13.4 & $1.2 \%$ & $37.5 \%$ & $24.8 \%$ & $36.5 \%$ & & $\mathrm{C}, \mathrm{D}$ \\
\hline Equally attracted to males \& females ${ }^{\mathrm{C}}$ & 546 & 4.2 & $1.8 \%$ & $31.1 \%$ & $21.1 \%$ & $46.0 \%$ & & $\mathrm{~A}, \mathrm{~B}, \mathrm{D}$ \\
\hline Mostly attracted to same $\operatorname{sex}^{\mathrm{D}}$ & 132 & 1.0 & NA & $28.0 \%$ & $33.3 \%$ & $38.6 \%$ & & $\mathrm{~B}, \mathrm{C}$ \\
\hline Only attracted to same $\operatorname{sex}^{\mathrm{E}}$ & 187 & 1.5 & $1.6 \%$ & $33.2 \%$ & $24.1 \%$ & $41.2 \%$ & & \\
\hline \multicolumn{9}{|l|}{ Age Group } \\
\hline $25-34^{\mathrm{A}}$ & 7,116 & 55.2 & $1.7 \%$ & $37.4 \%$ & $25.1 \%$ & $35.9 \%$ & & $\mathrm{~B}$ \\
\hline \multirow[t]{3}{*}{$35-44^{B}$} & 5,779 & 44.8 & $1.0 \%$ & $32.5 \%$ & $26.6 \%$ & $40.0 \%$ & & A \\
\hline & \multicolumn{7}{|c|}{ Access to Usual Source of Healthcare } & \\
\hline & $\begin{array}{l}\text { Number in } \\
\text { thousands }\end{array}$ & $\begin{array}{c}\% \text { of Total } \\
\text { Sample }\end{array}$ & Yes & No & & & & $\begin{array}{c}\text { *Outcome sig. } \\
\text { differences }\end{array}$ \\
\hline Total Adult Sample & 12,895 & 100.0 & $86.1 \%$ & $13.9 \%$ & & & & \\
\hline Sexual Identity & & & & & & & & \\
\hline Heterosexual $^{\mathrm{A}}$ & 11,790 & 91.4 & $86.3 \%$ & $13.7 \%$ & & & & $\mathrm{C}$ \\
\hline Homosexual, gay, or lesbian ${ }^{B}$ & 282 & 2.2 & $83.7 \%$ & $16.3 \%$ & & & & \\
\hline Bisexual $^{\mathrm{C}}$ & 823 & 6.4 & $83.0 \%$ & $17.0 \%$ & & & & A \\
\hline Sexual Behavior & & & & & & & & \\
\hline No sexual experience with same-sex partner ${ }^{A}$ & 10,345 & 80.2 & $86.5 \%$ & $13.5 \%$ & & & & $\mathrm{~B}$ \\
\hline Sexual experience with same-sex partner ${ }^{B}$ & 2,550 & 19.8 & $84.3 \%$ & $15.7 \%$ & & & & A \\
\hline Sexual Attraction & & & & & & & & \\
\hline Only attracted to opposite $\operatorname{sex}^{\mathrm{A}}$ & 10,306 & 79.9 & $86.5 \%$ & $13.5 \%$ & & & & $\mathrm{~B}$ \\
\hline Mostly attracted to opposite $\operatorname{sex}^{B}$ & 1,724 & 13.4 & $84.5 \%$ & $15.6 \%$ & & & & A \\
\hline Equally attracted to males $\&$ females ${ }^{\mathrm{C}}$ & 546 & 4.2 & $83.7 \%$ & $16.3 \%$ & & & & \\
\hline Mostly attracted to same $\operatorname{sex}^{D}$ & 132 & 1.0 & $86.4 \%$ & $13.6 \%$ & & & & \\
\hline Only attracted to same $\operatorname{sex}^{\mathrm{E}}$ & 187 & 1.5 & $81.8 \%$ & $18.2 \%$ & & & & \\
\hline Age Group & & & & & & & & \\
\hline $25-34^{\mathrm{A}}$ & 7,116 & 55.2 & $83.4 \%$ & $16.6 \%$ & & & & B \\
\hline $35-44^{\mathrm{B}}$ & 5,779 & 44.8 & $89.4 \%$ & $10.6 \%$ & & & & A \\
\hline
\end{tabular}

Note. Table presents unweighted data analyses. Row totals may not equal 100.0 due to rounding.

This sample does not include pregnant females or those missing values listwise on these and other variables explored in later regression analyses.

*Items in same greyscale were grouped together in above Pearson $\chi^{2}$ tests due to small cell sizes and prior examples in the literature.

Superscripts denote a Pearson $\chi^{2}$ test of $p<0.05$ between that category and its corresponding superscript. Fisher's exact two-tailed test also run for $2 \times 2$ tables; results are consistent. 
TABLE 2-E. CH. 2 REGRESSIONS: IDENTITY: FEMALE

Table 2-E. Regressions of Health Outcomes on Sexual Identity for Female Respondents Age 25-45 (N=12,895)

\begin{tabular}{|c|c|c|c|c|c|c|c|c|}
\hline & \multicolumn{2}{|c|}{ Self-Rated Health } & \multicolumn{2}{|c|}{ Overweight } & \multicolumn{2}{|c|}{ Obese } & \multicolumn{2}{|c|}{ Usual Source of Care } \\
\hline & OR & $95 \%$ CI & RRR & $95 \% \mathrm{CI}$ & RRR & $95 \% \mathrm{CI}$ & OR & $95 \%$ CI \\
\hline Intercept & $10.64 * * *$ & $(6.59,17.19)$ & $0.58 * *$ & $(0.42,0.81)$ & 0.81 & $(0.58,1.13)$ & $6.25 * * *$ & $(4.28,9.14)$ \\
\hline \multicolumn{9}{|l|}{ Sexual Identity } \\
\hline Heterosexual (ref) & 1.0 & & 1.0 & & 1.0 & & 1.0 & \\
\hline Homosexual, gay, or lesbian & 0.58 & $(0.31,1.11)$ & 1.17 & $(0.71,1.93)$ & 1.03 & $(0.66,1.60)$ & 0.72 & $(0.41,1.27)$ \\
\hline Bisexual & $0.62 * *$ & $(0.47,0.82)$ & 1.19 & $(0.90,1.59)$ & 1.18 & $(0.91,1.53)$ & 0.75 & $(0.55,1.01)$ \\
\hline \multicolumn{9}{|l|}{ Survey Cycle } \\
\hline 2011-2013 (ref) & 1.0 & & 1.0 & & 1.0 & & 1.0 & \\
\hline 2013-2015 & 0.81 & $(0.66,1.00)$ & 0.85 & $(0.71,1.02)$ & 0.99 & $(0.84,1.17)$ & 1.11 & $(0.89,1.39)$ \\
\hline 2015-2017 & 0.76 & $(0.58,1.00)$ & 0.92 & $(0.77,1.11)$ & 1.09 & $(0.89,1.33)$ & 1.05 & $(0.82,1.35)$ \\
\hline 2017-2019 & $0.78^{*}$ & $(0.61,0.98)$ & 1.11 & $(0.93,1.33)$ & $1.32 * *$ & $(1.10,1.58)$ & 0.79 & $(0.61,1.02)$ \\
\hline \multicolumn{9}{|l|}{ Age } \\
\hline 25-34 (ref) & 1.0 & & 1.0 & & 1.0 & & 1.0 & \\
\hline $35-44$ & $0.52 * * *$ & $(0.44,0.62)$ & $1.33 * * *$ & $(1.16,1.52)$ & $1.63^{* * *}$ & $(1.43,1.85)$ & $1.61 * * *$ & $(1.35,1.93)$ \\
\hline \multicolumn{9}{|l|}{ Race/ethnicity } \\
\hline White, non-Hispanic (ref) & 1.0 & & 1.0 & & 1.0 & & 1.0 & \\
\hline Hispanic & 0.87 & $(0.66,1.14)$ & $1.70 * * *$ & $(1.40,2.06)$ & $1.65^{* * *}$ & $(1.36,2.00)$ & 0.89 & $(0.70,1.12)$ \\
\hline Black, non-Hispanic & $1.33^{*}$ & $(1.03,1.71)$ & $2.04 * * *$ & $(1.67,2.49)$ & $3.03 * * *$ & $(2.49,3.68)$ & 1.10 & $(0.87,1.39)$ \\
\hline Other, non-Hispanic & 0.87 & $(0.60,1.27)$ & 0.94 & $(0.73,1.21)$ & 0.96 & $(0.75,1.25)$ & 0.87 & $(0.64,1.18)$ \\
\hline \multicolumn{9}{|l|}{ Nativity } \\
\hline US-born (ref) & 1.0 & & 1.0 & & 1.0 & & 1.0 & \\
\hline Foreign-born & $1.53 * *$ & $(1.15,2.03)$ & $0.71 * *$ & $(0.58,0.87)$ & $0.39 * * *$ & $(0.31,0.50)$ & $0.66 * * *$ & $(0.53,0.82)$ \\
\hline \multicolumn{9}{|l|}{ Residence } \\
\hline Principal MSA city (ref) & 1.0 & & 1.0 & & 1.0 & & 1.0 & \\
\hline Other MSA & 1.03 & $(0.84,1.27)$ & 1.09 & $(0.93,1.27)$ & $1.19^{*}$ & $(1.03,1.38)$ & 1.17 & $(0.98,1.40)$ \\
\hline Not MSA & 0.91 & $(0.69,1.18)$ & 1.11 & $(0.88,1.41)$ & $1.40^{* *}$ & $(1.10,1.78)$ & $1.46^{* *}$ & $(1.15,1.85)$ \\
\hline \multicolumn{9}{|l|}{ Education } \\
\hline Some high school or less (ref) & 1.0 & & 1.0 & & 1.0 & & 1.0 & \\
\hline High school diploma or GED & $1.99 * * *$ & $(1.56,2.55)$ & 1.05 & $(0.80,1.37)$ & 0.88 & $(0.69,1.12)$ & 1.09 & $(0.85,1.39)$ \\
\hline Some college & $1.90 * * *$ & $(1.43,2.53)$ & 0.91 & $(0.68,1.21)$ & 0.82 & $(0.63,1.05)$ & 1.18 & $(0.91,1.54)$ \\
\hline College or graduate degree & $3.25 * * *$ & $(2.43,4.35)$ & $0.64 * *$ & $(0.48,0.85)$ & $0.47 * * *$ & $(0.37,0.60)$ & 1.02 & $(0.78,1.32)$ \\
\hline \multicolumn{9}{|l|}{ Insurance } \\
\hline Private or Medi-Gap (ref) & 1.0 & & 1.0 & & 1.0 & & 1.0 & \\
\hline Medicaid, CHIP, State-sponsored & $0.59 * * *$ & $(0.45,0.78)$ & 1.12 & $(0.89,1.42)$ & 1.19 & $(0.97,1.46)$ & 0.78 & $(0.58,1.05)$ \\
\hline Medicare, military, government & $0.44 * * *$ & $(0.29,0.67)$ & 1.05 & $(0.77,1.44)$ & 0.98 & $(0.75,1.28)$ & 1.12 & $(0.75,1.65)$ \\
\hline Single-service, Indian Health Service, not covered & $0.67^{* *}$ & $(0.52,0.86)$ & 1.03 & $(0.85,1.25)$ & 1.16 & $(0.94,1.44)$ & $0.22 * * *$ & $(0.18,0.27)$ \\
\hline \multicolumn{9}{|l|}{ Household income, \% of federal poverty level } \\
\hline 0-99 (ref) & 1.0 & & 1.0 & & 1.0 & & 1.0 & \\
\hline $100-299$ & $1.38^{* *}$ & $(1.09,1.74)$ & 1.14 & $(0.95,1.38)$ & 1.03 & $(0.87,1.22)$ & 1.04 & $(0.85,1.27)$ \\
\hline$>\mathbf{3 0 0} \%$ & $2.65 * * *$ & $(1.91,3.66)$ & 1.06 & $(0.85,1.33)$ & $0.70 * *$ & $(0.57,0.86)$ & $1.37^{*}$ & $(1.05,1.80)$ \\
\hline \multicolumn{9}{|l|}{ Access to Usual Source of Care } \\
\hline Yes (ref) & 1.0 & & 1.0 & & 1.0 & & & \\
\hline No & 0.85 & $(0.65,1.11)$ & $0.77 *$ & $(0.63,0.95)$ & 0.83 & $(0.68,1.03)$ & & \\
\hline \multicolumn{9}{|l|}{ BMI } \\
\hline $18.5-24.9$ (Normal) (ref) & 1.0 & & & & & & 1.0 & \\
\hline$<18.5$ (Underweight) & 0.53 & $(0.24,1.21)$ & & & & & 1.19 & $(0.68,2.06)$ \\
\hline 25.0-29.9 (Overweight) & 1.05 & $(0.79,1.40)$ & & & & & $1.30 *$ & $(1.06,1.61)$ \\
\hline$>30$ (Obese) & $0.51 * * *$ & $(0.41,0.64)$ & & & & & 1.22 & $(0.99,1.51)$ \\
\hline
\end{tabular}

Note. Sample does not include pregnant females or those missing values listwise on these variables.

Self-rated general health $=0$ ) Poor, Fair 1) Good, Very good, Excellent. Logistic regression

The base outcome for the overweight and obese models is body mass index (BMI) that is low/normal ( $<25)$; overweight BMI is $25-29$, and obese BMI is $>30$. Multinomial regression.

Access to a usual source of care $=0$ ) No 1) Yes. Logistic regression.

$\mathrm{OR}=$ odds ratio $\mathrm{RRR}=$ relative risk ratio $\mathrm{CI}=$ confidence interval $\mathrm{MSA}=$ metropolitan statistical area .

The $p$-values are determined by the "Pearson $\chi^{2}$ test with the Rao and Scott $(1981,1984)$ second-order correction" or the $F$ test (the default output from the Stata svy tabulate command); ${ }^{*} p<0.05, * * p<0.01, * * * p<0.001$ 
TABLE 2-F. CH. 2 REGRESSIONS: ATTRACTION: FEMALE

Table 2-F. Regressions of Health Outcomes on Sexual Attraction for Female Respondents Age 25-45 (N=12,895)

\begin{tabular}{|c|c|c|c|c|c|c|c|c|}
\hline & \multicolumn{2}{|c|}{ Self-Rated Health } & \multicolumn{2}{|c|}{ Overweight } & \multicolumn{2}{|c|}{ Obese } & \multicolumn{2}{|c|}{ Usual Source of Care } \\
\hline & OR & $95 \%$ CI & RRR & $95 \% \mathrm{CI}$ & RRR & $95 \% \mathrm{CI}$ & OR & $95 \%$ CI \\
\hline Intercept & $\mathrm{Z}$ & $(6.75,17.67)$ & $0.60 * *$ & $(0.44,0.84)$ & 0.82 & $(0.59,1.14)$ & $6.43 * * *$ & $(4.42,9.35)$ \\
\hline \multicolumn{9}{|l|}{ Sexual Attraction } \\
\hline Only attracted to opposite sex (ref) & 1.0 & & 1.0 & & 1.0 & & 1.0 & \\
\hline Mostly attracted to opposite sex & 0.80 & $(0.61,1.04)$ & 0.86 & $(0.71,1.06)$ & 0.98 & $(0.81,1.19)$ & $0.81 *$ & $(0.67,0.99)$ \\
\hline Equally attracted to males $\&$ females & $0.59 * *$ & $(0.44,0.81)$ & 0.86 & $(0.60,1.22)$ & 1.11 & $(0.84,1.47)$ & 0.78 & $(0.52,1.17)$ \\
\hline Mostly attracted to same sex & 0.70 & $(0.30,1.62)$ & 1.39 & $(0.66,2.93)$ & 1.16 & $(0.59,2.28)$ & 0.67 & $(0.23,1.95)$ \\
\hline Only attracted to same sex & 0.49 & $(0.23,1.04)$ & 1.15 & $(0.60,2.22)$ & 1.02 & $(0.57,1.81)$ & 0.68 & $(0.39,1.17)$ \\
\hline \multicolumn{9}{|l|}{ Survey Cycle } \\
\hline 2011-2013 (ref) & 1.0 & & 1.0 & & 1.0 & & 1.0 & \\
\hline 2013-2015 & 0.81 & $(0.66,1.01)$ & 0.85 & $(0.71,1.03)$ & 0.99 & $(0.84,1.17)$ & 1.12 & $(0.89,1.40)$ \\
\hline 2015-2017 & 0.76 & $(0.58,1.00)$ & 0.92 & $(0.77,1.11)$ & 1.09 & $(0.89,1.34)$ & 1.05 & $(0.82,1.35)$ \\
\hline 2017-2019 & $0.78^{*}$ & $(0.62,0.98)$ & 1.12 & $(0.94,1.35)$ & $1.32 * *$ & $(1.11,1.58)$ & 0.79 & $(0.62,1.02)$ \\
\hline \multicolumn{9}{|l|}{ Age } \\
\hline 25-34 (ref) & 1.0 & & 1.0 & & 1.0 & & 1.0 & \\
\hline $35-44$ & $0.52 * * *$ & $(0.43,0.62)$ & $1.31^{* * *}$ & $(1.14,1.49)$ & $1.62 * * *$ & $(1.42,1.84)$ & $1.60^{* * *}$ & $(1.34,1.90)$ \\
\hline \multicolumn{9}{|l|}{ Race/ethnicity } \\
\hline White, non-Hispanic (ref) & 1.0 & & 1.0 & & 1.0 & & 1.0 & \\
\hline Hispanic & 0.87 & $(0.66,1.14)$ & $1.69 * * *$ & $(1.39,2.04)$ & $1.64 * * *$ & $(1.36,1.99)$ & 0.88 & $(0.70,1.12)$ \\
\hline Black, non-Hispanic & $1.31^{*}$ & $(1.02,1.68)$ & $2.01 * * *$ & $(1.65,2.46)$ & $3.02 * * *$ & $(2.48,3.67)$ & 1.08 & $(0.86,1.36)$ \\
\hline Other, non-Hispanic & 0.87 & $(0.60,1.27)$ & 0.94 & $(0.72,1.21)$ & 0.96 & $(0.74,1.25)$ & 0.86 & $(0.63,1.17)$ \\
\hline \multicolumn{9}{|l|}{ Nativity } \\
\hline US-born (ref) & 1.0 & & 1.0 & & 1.0 & & 1.0 & \\
\hline Foreign-born & $1.50 * *$ & $(1.13,2.00)$ & $0.70 * * *$ & $(0.57,0.85)$ & $0.39 * * *$ & $(0.31,0.49)$ & $0.65^{* * *}$ & $(0.52,0.81)$ \\
\hline \multicolumn{9}{|l|}{ Residence } \\
\hline Principal MSA city (ref) & 1.0 & & 1.0 & & 1.0 & & 1.0 & \\
\hline Other MSA & 1.02 & $(0.83,1.26)$ & 1.08 & $(0.93,1.26)$ & $1.19^{*}$ & $(1.03,1.38)$ & 1.16 & $(0.97,1.39)$ \\
\hline Not MSA & 0.90 & $(0.69,1.17)$ & 1.10 & $(0.87,1.39)$ & $1.39 * *$ & $(1.09,1.77)$ & $1.45^{* *}$ & $(1.14,1.83)$ \\
\hline \multicolumn{9}{|l|}{ Education } \\
\hline Some high school or less (ref) & 1.0 & & 1.0 & & 1.0 & & 1.0 & \\
\hline High school diploma or GED & $2.01 * * *$ & $(1.58,2.57)$ & 1.05 & $(0.80,1.38)$ & 0.88 & $(0.69,1.12)$ & 1.09 & $(0.85,1.40)$ \\
\hline Some college & $1.93 * * *$ & $(1.45,2.58)$ & 0.91 & $(0.68,1.22)$ & 0.82 & $(0.63,1.06)$ & 1.19 & $(0.92,1.55)$ \\
\hline College or graduate degree & $3.29 * * *$ & $(2.46,4.42)$ & $0.64 * *$ & $(0.48,0.85)$ & $0.47 * * *$ & $(0.37,0.60)$ & 1.03 & $(0.79,1.34)$ \\
\hline \multicolumn{9}{|l|}{ Insurance } \\
\hline Private or Medi-Gap (ref) & 1.0 & & 1.0 & & 1.0 & & 1.0 & \\
\hline Medicaid, CHIP, State-sponsored & $0.59 * * *$ & $(0.45,0.78)$ & 1.14 & $(0.90,1.44)$ & 1.19 & $(0.97,1.47)$ & 0.78 & $(0.58,1.05)$ \\
\hline Medicare, military, government & $0.45^{* * *}$ & $(0.29,0.67)$ & 1.05 & $(0.77,1.45)$ & 0.98 & $(0.74,1.28)$ & 1.12 & $(0.76,1.65)$ \\
\hline Single-service, Indian Health Service, not covered & $0.67 * *$ & $(0.52,0.86)$ & 1.04 & $(0.85,1.26)$ & 1.17 & $(0.95,1.44)$ & $0.22 * * *$ & $(0.18,0.27)$ \\
\hline \multicolumn{9}{|l|}{ Household income, \% of federal poverty level } \\
\hline $0-99$ (ref) & 1.0 & & 1.0 & & 1.0 & & 1.0 & \\
\hline $100-299$ & $1.38 * *$ & $(1.08,1.75)$ & 1.15 & $(0.95,1.39)$ & 1.03 & $(0.87,1.22)$ & 1.04 & $(0.85,1.27)$ \\
\hline$>\mathbf{3 0 0} \%$ & $2.65 * * *$ & $(1.91,3.68)$ & 1.07 & $(0.85,1.34)$ & $0.70 * *$ & $(0.57,0.86)$ & $1.38 *$ & $(1.05,1.81)$ \\
\hline \multicolumn{9}{|l|}{ Access to Usual Source of Care } \\
\hline Yes (ref) & 1.0 & & 1.0 & & 1.0 & & & \\
\hline No & 0.85 & $(0.65,1.11)$ & $0.78^{*}$ & $(0.63,0.96)$ & 0.84 & $(0.68,1.03)$ & & \\
\hline \multicolumn{9}{|l|}{ BMI } \\
\hline 18.5-24.9 (Normal) (ref) & 1.0 & & & & & & 1.0 & \\
\hline$<18.5$ (Underweight) & 0.53 & $(0.24,1.21)$ & & & & & 1.17 & $(0.68,2.04)$ \\
\hline 25.0-29.9 (Overweight) & 1.04 & $(0.78,1.38)$ & & & & & $1.30^{*}$ & $(1.05,1.60)$ \\
\hline$>30$ (Obese) & $0.51 * * *$ & $(0.41,0.64)$ & & & & & 1.22 & $(0.98,1.50)$ \\
\hline
\end{tabular}

Note. Sample does not include pregnant females or those missing values listwise on these variables.

Self-rated general health $=0$ ) Poor, Fair 1) Good, Very good, Excellent. Logistic regression

The base outcome for the overweight and obese models is body mass index (BMI) that is low/normal (<25); overweight BMI is 25-29, and obese BMI is $>30$. Multinomial regression.

Access to a usual source of care $=0$ ) No 1) Yes. Logistic regression.

$\mathrm{OR}=$ odds ratio $; \mathrm{RRR}=$ relative risk ratio $\mathrm{CI}=$ confidence interval $; \mathrm{MSA}=$ metropolitan statistical area.

The $p$-values are determined by the "Pearson $\chi^{2}$ test with the Rao and Scott $(1981,1984)$ second-order correction" or the $F$ test (the default output from the Stata svy tabulate command); ${ }^{*} p<0.05,{ }^{* *} p<0.01,{ }^{* * *} p<0.001$ 
TABLE 2-G. CH. 2 REGRESSIONS: BEHAVIOR: FEMALE

Table 2-G. Regressions of Health Outcomes on Sexual Behavior for Female Respondents Age 25-45 (N=12,895)

\begin{tabular}{|c|c|c|c|c|c|c|c|c|}
\hline & \multicolumn{2}{|c|}{ Self-Rated Health } & \multicolumn{2}{|c|}{ Overweight } & \multicolumn{2}{|c|}{ Obese } & \multicolumn{2}{|c|}{ Usual Source of Care } \\
\hline & OR & $95 \% \mathrm{CI}$ & RRR & $95 \% \mathrm{CI}$ & RRR & $95 \% \mathrm{CI}$ & OR & $95 \% \mathrm{CI}$ \\
\hline Intercept & $10.80^{* * *}$ & $(6.60,17.69)$ & $0.59 * *$ & $(0.43,0.82)$ & 0.82 & $(0.59,1.13)$ & $6.18 * * *$ & $(4.23,9.03)$ \\
\hline \multicolumn{9}{|l|}{ Sexual Behavior } \\
\hline No sexual experience with same-sex partner (ref) & 1.0 & & 1.0 & & 1.0 & & 1.0 & \\
\hline Sexual experience with same-sex partner & $0.73 * *$ & $(0.58,0.91)$ & 1.03 & $(0.85,1.26)$ & 1.03 & $(0.87,1.21)$ & 0.91 & $(0.74,1.11)$ \\
\hline \multicolumn{9}{|l|}{ Survey Cycle } \\
\hline 2011-2013 (ref) & 1.0 & & 1.0 & & 1.0 & & 1.0 & \\
\hline 2013-2015 & 0.81 & $(0.66,1.00)$ & 0.85 & $(0.71,1.03)$ & 0.99 & $(0.84,1.17)$ & 1.11 & $(0.89,1.39)$ \\
\hline 2015-2017 & $0.76^{*}$ & $(0.58,0.99)$ & 0.92 & $(0.77,1.11)$ & 1.09 & $(0.89,1.34)$ & 1.05 & $(0.82,1.34)$ \\
\hline 2017-2019 & $0.77 *$ & $(0.61,0.97)$ & 1.12 & $(0.93,1.34)$ & $1.32 * *$ & $(1.11,1.58)$ & 0.78 & $(0.61,1.00)$ \\
\hline \multicolumn{9}{|l|}{ Age } \\
\hline 25-34 (ref) & 1.0 & & 1.0 & & 1.0 & & 1.0 & \\
\hline $35-44$ & $0.53 * * *$ & $(0.44,0.63)$ & $1.32 * * *$ & $(1.16,1.51)$ & $1.62 * * *$ & $(1.42,1.84)$ & $1.63 * * *$ & $(1.36,1.94)$ \\
\hline \multicolumn{9}{|l|}{ Race/ethnicity } \\
\hline White, non-Hispanic (ref) & 1.0 & & 1.0 & & 1.0 & & 1.0 & \\
\hline Hispanic & 0.86 & $(0.65,1.14)$ & $1.70 * * *$ & $(1.40,2.05)$ & $1.64 * * *$ & $(1.36,1.99)$ & 0.89 & $(0.70,1.13)$ \\
\hline Black, non-Hispanic & $1.31 *$ & $(1.02,1.68)$ & $2.04 * * *$ & $(1.66,2.49)$ & $3.02 * * *$ & $(2.49,3.67)$ & 1.10 & $(0.87,1.39)$ \\
\hline Other, non-Hispanic & 0.86 & $(0.60,1.25)$ & 0.94 & $(0.73,1.22)$ & 0.96 & $(0.75,1.25)$ & 0.86 & $(0.63,1.18)$ \\
\hline \multicolumn{9}{|l|}{ Nativity } \\
\hline US-born (ref) & 1.0 & & 1.0 & & 1.0 & & 1.0 & \\
\hline Foreign-born & $1.49 * *$ & $(1.12,1.98)$ & $0.71 * *$ & $(0.58,0.87)$ & $0.39 * * *$ & $(0.31,0.49)$ & $0.66 * * *$ & $(0.53,0.82)$ \\
\hline \multicolumn{9}{|l|}{ Residence } \\
\hline Principal MSA city (ref) & 1.0 & & 1.0 & & 1.0 & & 1.0 & \\
\hline Other MSA & 1.02 & $(0.83,1.27)$ & 1.08 & $(0.93,1.27)$ & $1.19^{*}$ & $(1.03,1.38)$ & 1.17 & $(0.98,1.40)$ \\
\hline Not MSA & 0.90 & $(0.69,1.18)$ & 1.11 & $(0.87,1.40)$ & $1.39 * *$ & $(1.09,1.77)$ & $1.47 * *$ & $(1.16,1.86)$ \\
\hline \multicolumn{9}{|l|}{ Education } \\
\hline Some high school or less (ref) & 1.0 & & 1.0 & & 1.0 & & 1.0 & \\
\hline High school diploma or GED & $2.01 * * *$ & $(1.57,2.56)$ & 1.05 & $(0.80,1.37)$ & 0.88 & $(0.69,1.12)$ & 1.09 & $(0.85,1.40)$ \\
\hline Some college & $1.93 * * *$ & $(1.45,2.57)$ & 0.91 & $(0.68,1.21)$ & 0.82 & $(0.63,1.05)$ & 1.19 & $(0.91,1.55)$ \\
\hline College or graduate degree & $3.28 * * *$ & $(2.45,4.39)$ & $0.64 * *$ & $(0.48,0.85)$ & $0.47 * * *$ & $(0.37,0.60)$ & 1.02 & $(0.79,1.33)$ \\
\hline \multicolumn{9}{|l|}{ Insurance } \\
\hline Private or Medi-Gap (ref) & 1.0 & & 1.0 & & 1.0 & & 1.0 & \\
\hline Medicaid, CHIP, State-sponsored & $0.60 * * *$ & $(0.46,0.78)$ & 1.13 & $(0.89,1.42)$ & 1.19 & $(0.97,1.47)$ & 0.78 & $(0.58,1.05)$ \\
\hline Medicare, military, government & $0.44 * * *$ & $(0.29,0.67)$ & 1.05 & $(0.77,1.45)$ & 0.98 & $(0.75,1.28)$ & 1.11 & $(0.75,1.65)$ \\
\hline Single-service, Indian Health Service, not covered & $0.67 * *$ & $(0.52,0.86)$ & 1.03 & $(0.85,1.25)$ & 1.17 & $(0.94,1.44)$ & $0.22 * * *$ & $(0.18,0.27)$ \\
\hline \multicolumn{9}{|l|}{ Household income, \% of federal poverty level } \\
\hline $0-99$ (ref) & 1.0 & & 1.0 & & 1.0 & & 1.0 & \\
\hline $100-299$ & $1.37 * *$ & $(1.08,1.74)$ & 1.15 & $(0.95,1.38)$ & 1.03 & $(0.87,1.22)$ & 1.04 & $(0.85,1.27)$ \\
\hline$>\mathbf{3 0 0} \%$ & $2.63 * * *$ & $(1.91,3.64)$ & 1.06 & $(0.85,1.33)$ & $0.70 * *$ & $(0.57,0.86)$ & $1.38 *$ & $(1.05,1.81)$ \\
\hline \multicolumn{9}{|l|}{ Access to Usual Source of Care } \\
\hline Yes (ref) & 1.0 & & 1.0 & & 1.0 & & & \\
\hline No & 0.86 & $(0.65,1.12)$ & $0.78 *$ & $(0.63,0.95)$ & 0.84 & $(0.68,1.03)$ & & \\
\hline \multicolumn{9}{|l|}{ BMI } \\
\hline 18.5-24.9 (Normal) (ref) & 1.0 & & & & & & 1.0 & \\
\hline$<18.5$ (Underweight) & 0.53 & $(0.23,1.21)$ & & & & & 1.19 & $(0.68,2.08)$ \\
\hline 25.0-29.9 (Overweight) & 1.05 & $(0.79,1.39)$ & & & & & $1.30 *$ & $(1.06,1.60)$ \\
\hline$>30.0$ (Obese) & $0.51 * * *$ & $(0.41,0.64)$ & & & & & 1.22 & $(0.99,1.50)$ \\
\hline
\end{tabular}

Note. Sample does not include pregnant females or those missing values listwise on these variables.

Self-rated general health $=0$ ) Poor, Fair 1) Good, Very good, Excellent. Logistic regression

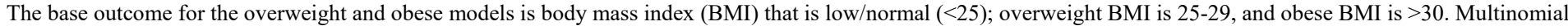
regression.

Access to a usual source of care $=0$ ) No 1) Yes. Logistic regression.

$\mathrm{OR}=$ odds ratio; $\mathrm{RRR}=$ relative risk ratio $\mathrm{CI}=$ confidence interval MSA $=$ metropolitan statistical area.

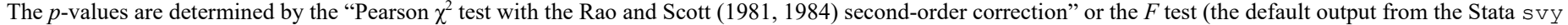

tabulate command); ${ }^{*} p<0.05, * * p<0.01, * * * p<0.001$ 
Table 2-H. Characteristics of US Males Aged 25-45 Years, by Sexual Identity 2011-2019 (N=10,672)

\begin{tabular}{|c|c|c|c|c|c|c|}
\hline & \multicolumn{6}{|c|}{ Sexual Identity } \\
\hline & \multicolumn{2}{|c|}{$\begin{array}{l}\text { Heterosexual or straight } \\
\quad(\mathrm{n}=10,208 ; 95.7 \%)^{\mathrm{a}}\end{array}$} & \multicolumn{2}{|c|}{$\begin{array}{l}\text { Homosexual or gay } \\
\quad(n=270 ; 2.5 \%)^{a}\end{array}$} & \multicolumn{2}{|c|}{$\begin{array}{c}\text { Bisexual } \\
(n=194 ; 1.8 \%)^{a}\end{array}$} \\
\hline & Mean \% & $95 \%$ CI & Mean \% & $95 \%$ CI & Mean \% & $95 \%$ CI \\
\hline \multicolumn{7}{|l|}{ Sexual Attraction*** } \\
\hline Only attracted to females & 96.7 & $(96.2,97.1)$ & 3.8 & $(1.4,9.8)$ & 11.6 & $(6.4,20.3)$ \\
\hline Mostly attracted to females & 3.1 & $(2.7,3.6)$ & 0.0 & $(\mathrm{~N} / \mathrm{A})$ & 41.1 & $(31.3,51.7)$ \\
\hline Equally attracted to males \& females & 0.1 & $(0.1,0.3)$ & 1.4 & $(0.5,3.9)$ & 33.7 & $(24.5,44.3)$ \\
\hline Mostly attracted to males & 0.0 & $(0.0,0.0)$ & 26.3 & $(17.9,36.9)$ & 13.3 & $(8.1,21.2)$ \\
\hline Only attracted to males & 0.1 & $(0.0,0.1)$ & 68.6 & $(58.3,77.4)$ & 0.3 & $(0.0,1.8)$ \\
\hline \multicolumn{7}{|l|}{ Sexual Behavior $* * *$} \\
\hline Sexual experience with same-sex partner & 2.8 & $(2.4,3.2)$ & 97.0 & $(93.7,98.6)$ & 81.7 & $(72.9,88.1)$ \\
\hline No sexual experience with same-sex partner & 97.2 & $(96.8,97.6)$ & 3.0 & $(1.4,6.3)$ & 18.3 & $(11.9,27.1)$ \\
\hline \multicolumn{7}{|l|}{ Age } \\
\hline 25-34 & 52.1 & $(50.5,53.7)$ & 53.9 & $(44.6,62.9)$ & 64.2 & $(55.0,72.5)$ \\
\hline 35-44 & 47.9 & $(46.3,49.5)$ & 46.1 & $(37.1,55.4)$ & 35.8 & $(27.5,45.0)$ \\
\hline \multicolumn{7}{|l|}{ Race/ethnicity } \\
\hline White, non-Hispanic & 58.4 & $(56.1,60.8)$ & 53.7 & $(44.0,63.1)$ & 62.9 & $(53.8,71.2)$ \\
\hline Hispanic & 20.2 & $(18.1,22.4)$ & 26.8 & $(17.8,38.3)$ & 21.3 & $(15.2,28.9)$ \\
\hline Black, non-Hispanic & 11.4 & $(10.1,12.8)$ & 6.9 & $(4.3,10.9)$ & 5.9 & $(3.4,10.1)$ \\
\hline Other, non-Hispanic & 10.0 & $(8.8,11.4)$ & 12.5 & $(7.8,19.6)$ & 9.9 & $(4.9,18.9)$ \\
\hline \multicolumn{7}{|l|}{ Residence $^{* *}$} \\
\hline Principal MSA city & 34.3 & $(31.3,37.5)$ & 48.2 & $(38.8,57.7)$ & 46.8 & $(36.8,57.0)$ \\
\hline Other MSA & 50.6 & $(47.2,54.0)$ & 40.6 & $(31.0,50.9)$ & 43.8 & $(34.1,54.0)$ \\
\hline Not MSA & 15.0 & $(12.0,18.8)$ & 11.2 & $(6.5,18.8)$ & 9.4 & $(5.5,15.7)$ \\
\hline \multicolumn{7}{|l|}{ Nativity } \\
\hline US-born & 81.1 & $(79.5,82.6)$ & 84.2 & $(76.9,89.6)$ & 85.3 & $(78.1,90.4)$ \\
\hline Foreign-born & 18.9 & $(17.4,20.5)$ & 15.8 & $(10.4,23.1)$ & 14.7 & $(9.6,21.9)$ \\
\hline \multicolumn{7}{|l|}{ Education* } \\
\hline Some high school or less & 11.0 & $(9.9,12.2)$ & 6.5 & $(3.4,12.0)$ & 11.6 & $(6.3,20.6)$ \\
\hline High school diploma or GED & 27.9 & $(26.4,29.5)$ & 19.1 & $(13.3,26.5)$ & 26.7 & $(19.0,36.3)$ \\
\hline Some college & 19.6 & $(18.4,20.8)$ & 18.3 & $(13.0,25.1)$ & 19.1 & $(13.0,27.2)$ \\
\hline College or graduate degree & 41.5 & $(39.4,43.7)$ & 56.1 & $(49.0,63.0)$ & 42.5 & $(32.8,52.9)$ \\
\hline \multicolumn{7}{|l|}{ Insurance* } \\
\hline Private or Medi-Gap & 65.4 & $(63.6,67.2)$ & 69.0 & $(60.5,76.4)$ & 59.3 & $(50.3,67.7)$ \\
\hline Medicaid, CHIP, State-sponsored & 8.5 & $(7.6,9.5)$ & 13.1 & $(8.4,20.0)$ & 11.5 & $(6.5,19.4)$ \\
\hline Medicare, military, government & 4.9 & $(4.1,5.9)$ & 4.0 & $(1.9,8.5)$ & 3.0 & $(1.6,5.7)$ \\
\hline Single-service, Indian Health Service, not covered & 21.2 & $(19.7,22.8)$ & 13.8 & $(9.7,19.3)$ & 26.2 & $(18.9,35.0)$ \\
\hline \multicolumn{7}{|l|}{ Household income, \% of federal poverty level* } \\
\hline $0-99$ & 14.0 & $(13.0,15.2)$ & 12.5 & $(8.4,18.3)$ & 22.8 & $(16.4,30.8)$ \\
\hline 100-299 & 35.8 & $(34.2,37.5)$ & 32.0 & $(24.6,40.5)$ & 37.3 & $(28.5,46.9)$ \\
\hline$>\mathbf{3 0 0} \%$ & 50.1 & $(48.2,52.1)$ & 55.4 & $(46.2,64.3)$ & 39.9 & $(30.6,50.1)$ \\
\hline \multicolumn{7}{|l|}{ Self-rated health } \\
\hline Poor & 0.9 & $(0.7,1.3)$ & 1.3 & $(0.4,4.1)$ & 0.6 & $(0.1,2.5)$ \\
\hline Fair & 6.0 & $(5.3,6.7)$ & 5.1 & $(2.9,9.0)$ & 8.8 & $(5.1,14.5)$ \\
\hline Good & 25.4 & $(24.3,26.6)$ & 20.9 & $(15.2,28.1)$ & 36.0 & $(27.3,45.8)$ \\
\hline Very good & 40.4 & $(38.9,41.8)$ & 40.3 & $(33.5,47.6)$ & 31.5 & $(22.9,41.7)$ \\
\hline Excellent & 27.3 & $(26.0,28.7)$ & 32.3 & $(24.9,40.8)$ & 23.0 & $(16.2,31.7)$ \\
\hline \multicolumn{7}{|l|}{ BMI** } \\
\hline$<18.5$ (Underweight) & 0.8 & $(0.6,1.1)$ & 0.5 & $(0.2,1.7)$ & 0.0 & (N/A) \\
\hline 18.5-24.9 (Normal) & 26.5 & $(25.3,27.9)$ & 39.7 & $(31.6,48.3)$ & 27.3 & $(18.5,38.3)$ \\
\hline 25.0-29.9 (Overweight) & 41.3 & $(39.9,42.7)$ & 29.4 & $(22.2,37.8$ & 33.6 & $(24.7,43.7)$ \\
\hline$>30.0$ (Obese) & 31.4 & $(30.0,32.7)$ & 30.4 & $(23.4,38.5)$ & 39.2 & $(29.9,49.2)$ \\
\hline \multicolumn{7}{|l|}{ Access to Usual Source of Care $* * *$} \\
\hline Yes & 70.6 & $(69.0,72.1)$ & 85.2 & $(78.8,89.9)$ & 75.6 & $(67.1,82.5)$ \\
\hline No & 29.4 & $(27.9,31.0)$ & 14.8 & $(10.1,21.2)$ & 24.4 & $(17.5,32.9)$ \\
\hline Cycle* & & & & & & \\
\hline 2011-2013 & 25.2 & $(23.0,27.6)$ & 21.0 & $(14.3,29.6)$ & 28.0 & $(20.0,37.6)$ \\
\hline 2013-2015 & 27.0 & $(24.4,29.7)$ & 16.3 & $(11.2,23.3)$ & 22.4 & $(15.3,31.6)$ \\
\hline 2015-2017 & 24.1 & $(21.8,26.5)$ & 34.4 & $(23.4,47.3)$ & 22.2 & $(15.9,30.2)$ \\
\hline 2017-2019 & 23.8 & $(21.4,26.3)$ & 28.3 & $(20.7,37.4)$ & 27.3 & $(19.0,37.7)$ \\
\hline
\end{tabular}

${ }^{\mathrm{a}} \mathrm{n}=$ sample number and percent without using svy command; Mean and 95\% CI were conducted using svy command.

${ }^{*} \mathrm{p}<0.05, *{ }^{*} \mathrm{p}<0.01, * * * \mathrm{p}<0.001 ; p$-values were determined using the "Pearson $\chi 2$ test with the Rao and Scott (1981, 1984) second-order correction" or the $F$ test (the default output from the Stata Svy tabulate command) (Stata.com n.d.:11).

Note. Sample does not include those missing values listwise on these variables for males or females. MSA = metropolitan statistical area; $\mathrm{BMI}=$ body mass index; $\mathrm{N} / \mathrm{A}=$ not applicable; not all strata included in each cell if missing subpopulation members. 
TABLE 2-I. CH. 2 SUMMARY STATISTICS: ATTRACTION: MALE

Table 2-I. Characteristics of US Males Aged 25-45 Years, by Sexual Attraction 2011-2019 (N=10,672)

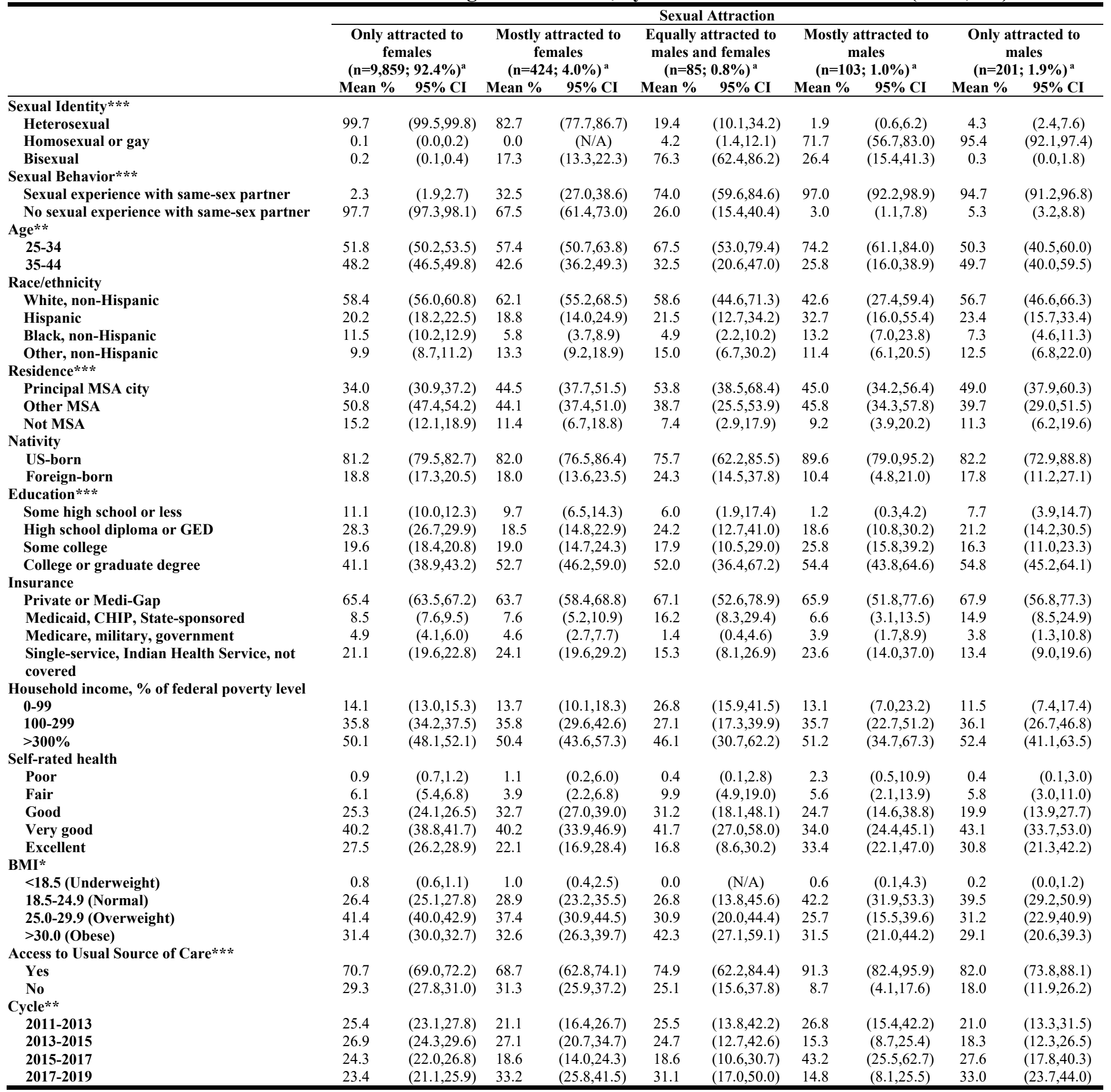

${ }^{a} \mathrm{n}=$ sample number and percent without using svy command; Mean and $95 \%$ CI were conducted using svy command.

${ }^{*} \mathrm{p}<0.05, * * \mathrm{p}<0.01,{ }^{* * *} \mathrm{p}<0.001 ; p$-values were determined using the "Pearson $\chi 2$ test with the Rao and Scott $(1981,1984)$ second-order correction" or the $F$ test (the default output from the Stata svy tabulate command) (Stata.com n.d.:11).

Note. Sample does not include those missing values listwise on these variables for males or females. MSA = metropolitan statistical area; $\mathrm{BMI}=$ body mass index; $\mathrm{N} / \mathrm{A}=$ not applicable; not all strata included in each cell if missing subpopulation members. 
Table 2-J. Characteristics of US Males Aged 25-45 Years, by Sexual Behavior 2011-2019 (N=10,672)

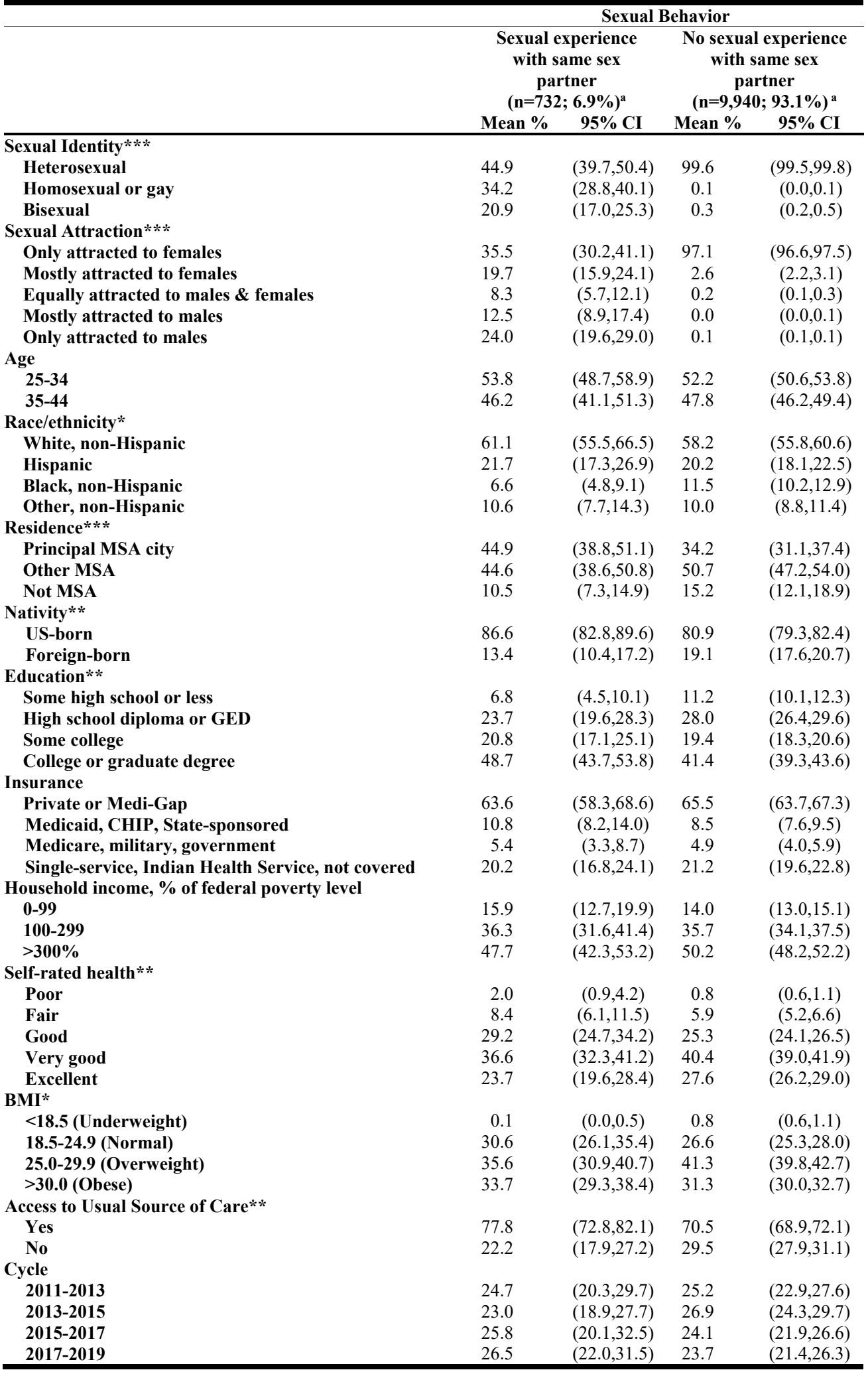

${ }^{a} \mathrm{n}=$ sample number and percent without using svy command; Mean and $95 \%$ CI were conducted using svy command.

${ }^{*} \mathrm{p}<0.05,{ }^{*} \mathrm{p}<0.01,{ }^{* * *} \mathrm{p}<0.001 ; p$-values were determined using the "Pearson $\chi 2$ test with the Rao and Scott (1981, 1984) second-order correction" or the $F$ test (the default output from the Stata svy tabulate command)

(Stata.com n.d.:11).

Note. Sample does not include those missing values listwise on these variables for males or females. MSA = metropolitan statistical area; $\mathrm{BMI}=$ body mass index; $\mathrm{N} / \mathrm{A}=$ not applicable; not all strata included in each cell if missing subpopulation members. 
TABLE 2-K. CH. 2 SUMMARY STATISTICS: COMPONENTS AND OUTCOMES: MALE

Table 2-K. Sample Characteristics of US Males by Sexual Identity Components and Health Outcomes 2011-2019 (N=10,672)

\begin{tabular}{|c|c|c|c|c|c|c|c|c|}
\hline & \multirow[b]{2}{*}{$\begin{array}{l}\text { Number in } \\
\text { thousands }\end{array}$} & \multirow[b]{2}{*}{$\begin{array}{c}\% \text { of Total } \\
\text { Sample } \\
\end{array}$} & \multicolumn{5}{|c|}{ Self-Rated Health } & \multirow[b]{2}{*}{$\begin{array}{c}\text { *Outcome sig. } \\
\text { differences }\end{array}$} \\
\hline & & & Poor & Fair & Good & Very Good & Excellent & \\
\hline Total Adult Sample & 10,672 & 100.0 & $1.1 \%$ & $6.5 \%$ & $25.8 \%$ & $39.7 \%$ & $26.9 \%$ & \\
\hline \multicolumn{9}{|l|}{ Sexual Identity } \\
\hline Heterosexual $^{\mathrm{A}}$ & 10,208 & 95.7 & $1.1 \%$ & $6.5 \%$ & $25.7 \%$ & $39.8 \%$ & $26.9 \%$ & \\
\hline Homosexual or gay $^{B}$ & 270 & 2.5 & $1.5 \%$ & $6.3 \%$ & $23.7 \%$ & $40.0 \%$ & $28.5 \%$ & \\
\hline Bisexual $^{\mathrm{C}}$ & 194 & 1.8 & $1.0 \%$ & $9.3 \%$ & $33.5 \%$ & $33.0 \%$ & $23.2 \%$ & \\
\hline \multicolumn{9}{|l|}{ Sexual Behavior } \\
\hline No sexual experience with same-sex partner ${ }^{A}$ & 9,940 & 93.1 & $1.1 \%$ & $6.4 \%$ & $25.5 \%$ & $39.9 \%$ & $27.1 \%$ & $\mathrm{~B}$ \\
\hline Sexual experience with same-sex partner ${ }^{B}$ & 732 & 6.9 & $1.8 \%$ & $8.7 \%$ & $29.6 \%$ & $36.6 \%$ & $23.2 \%$ & A \\
\hline \multicolumn{9}{|l|}{ Sexual Attraction } \\
\hline Only attracted to opposite sex ${ }^{A}$ & 9859 & 92.4 & $1.2 \%$ & $6.5 \%$ & $25.6 \%$ & $39.7 \%$ & $27.1 \%$ & \\
\hline Mostly attracted to opposite $\operatorname{sex}^{B}$ & 424 & 4.0 & $0.5 \%$ & $6.1 \%$ & $31.6 \%$ & $40.1 \%$ & $21.7 \%$ & $\mathrm{C}^{* *}$ \\
\hline Equally attracted to males $\&$ females ${ }^{C}$ & 85 & 0.8 & $1.2 \%$ & $11.8 \%$ & $27.1 \%$ & $40.0 \%$ & $20.0 \%$ & $\mathrm{~B}^{* *}$ \\
\hline Mostly attracted to same $\operatorname{sex}^{D}$ & 103 & 1.0 & $1.9 \%$ & $5.8 \%$ & $27.2 \%$ & $35.9 \%$ & $29.1 \%$ & \\
\hline Only attracted to same $\operatorname{sex}^{\mathrm{E}}$ & 201 & 1.9 & $0.5 \%$ & $7.0 \%$ & $23.4 \%$ & $41.8 \%$ & $27.4 \%$ & \\
\hline \multicolumn{9}{|l|}{ Age Group } \\
\hline $25-34^{\mathrm{A}}$ & 5,922 & 55.5 & $0.9 \%$ & $4.9 \%$ & $24.2 \%$ & $40.3 \%$ & $29.8 \%$ & $\mathrm{~B}$ \\
\hline \multirow[t]{3}{*}{$35-44^{B}$} & 4,750 & 44.5 & $1.4 \%$ & $8.6 \%$ & $27.8 \%$ & $39.0 \%$ & $23.3 \%$ & A \\
\hline & & \multicolumn{7}{|c|}{ Body Mass Index (BMI) } \\
\hline & $\begin{array}{l}\text { Number in } \\
\text { thousands }\end{array}$ & $\begin{array}{c}\% \text { of Total } \\
\text { Sample }\end{array}$ & $\begin{array}{c}<18.5 \\
\text { (Underweight) }\end{array}$ & $\begin{array}{c}20-24 \\
\text { (Normal) }\end{array}$ & $\begin{array}{c}25.0-29.9 \\
\text { (Overweight) }\end{array}$ & $\begin{array}{c}\text { 30-50 } \\
\text { (Obese) }\end{array}$ & & $\begin{array}{c}\text { *Outcome sig. } \\
\text { differences }\end{array}$ \\
\hline Total Adult Sample & 10,672 & 100.0 & $0.8 \%$ & $28.4 \%$ & $39.2 \%$ & $31.6 \%$ & & \\
\hline \multicolumn{9}{|l|}{ Sexual Identity } \\
\hline Heterosexual $^{\mathrm{A}}$ & 10,208 & 95.7 & $0.8 \%$ & $28.1 \%$ & $39.5 \%$ & $31.6 \%$ & & $\mathrm{~B}$ \\
\hline Homosexual or gay ${ }^{B}$ & 270 & 2.5 & $1.1 \%$ & $39.6 \%$ & $32.2 \%$ & $27.0 \%$ & & $\mathrm{~A}, \mathrm{C}$ \\
\hline Bisexual $^{\mathrm{C}}$ & 194 & 1.8 & $0.0 \%$ & $27.3 \%$ & $33.5 \%$ & $39.2 \%$ & & B \\
\hline \multicolumn{9}{|l|}{ Sexual Behavior } \\
\hline No sexual experience with same-sex partner ${ }^{A}$ & 9,940 & 93.1 & $0.9 \%$ & $28.2 \%$ & $39.4 \%$ & $31.6 \%$ & & \\
\hline Sexual experience with same-sex partner ${ }^{B}$ & 732 & 6.9 & $0.3 \%$ & $31.4 \%$ & $35.9 \%$ & $32.4 \%$ & & \\
\hline \multicolumn{9}{|l|}{ Sexual Attraction } \\
\hline Only attracted to opposite sex ${ }^{A}$ & 9859 & 92.4 & $0.8 \%$ & $28.1 \%$ & $39.6 \%$ & $31.5 \%$ & & $\mathrm{D}, \mathrm{E}$ \\
\hline Mostly attracted to opposite $\operatorname{sex}^{B}$ & 424 & 4.0 & $1.2 \%$ & $28.5 \%$ & $35.6 \%$ & $34.7 \%$ & & $\mathrm{D}, \mathrm{E}$ \\
\hline Equally attracted to males $\&$ females ${ }^{\mathrm{C}}$ & 85 & 0.8 & $0.0 \%$ & $22.4 \%$ & $35.3 \%$ & $42.4 \%$ & & $\mathrm{D}, \mathrm{E}$ \\
\hline Mostly attracted to same $\operatorname{sex}^{\mathrm{D}}$ & 103 & 1.0 & $1.0 \%$ & $43.7 \%$ & $26.2 \%$ & $29.1 \%$ & & $\mathrm{~A}, \mathrm{~B}, \mathrm{C}$ \\
\hline Only attracted to same $\operatorname{sex}^{\mathrm{E}}$ & 201 & 1.9 & $0.5 \%$ & $39.8 \%$ & $33.3 \%$ & $26.4 \%$ & & $\mathrm{~A}, \mathrm{~B}, \mathrm{C}$ \\
\hline \multicolumn{9}{|l|}{ Age Group } \\
\hline $25-34^{A}$ & 5,922 & 55.5 & $1.0 \%$ & $32.5 \%$ & $37.7 \%$ & $28.9 \%$ & & B \\
\hline \multirow[t]{3}{*}{$35-44^{\text {B }}$} & 4,750 & 44.5 & $0.7 \%$ & $23.3 \%$ & $41.0 \%$ & $35.0 \%$ & & A \\
\hline & \multicolumn{7}{|c|}{ Access to Usual Source of Healthcare } & \\
\hline & $\begin{array}{l}\text { Number in } \\
\text { thousands }\end{array}$ & $\begin{array}{c}\% \text { of Total } \\
\text { Sample }\end{array}$ & Yes & No & & & & $\begin{array}{c}* \text { Outcome sig. } \\
\text { differences }\end{array}$ \\
\hline Total Adult Sample & 10,672 & 100.0 & $69.7 \%$ & $30.3 \%$ & & & & \\
\hline Sexual Identity & & & & & & & & \\
\hline Heterosexual $^{\mathrm{A}}$ & 10,208 & 95.7 & $69.3 \%$ & $30.7 \%$ & & & & B \\
\hline Homosexual or gay ${ }^{B}$ & 270 & 2.5 & $82.2 \%$ & $17.8 \%$ & & & & $\mathrm{~A}, \mathrm{C}$ \\
\hline Bisexual $^{\mathrm{C}}$ & 194 & 1.8 & $72.2 \%$ & $27.8 \%$ & & & & B \\
\hline Sexual Behavior & & & & & & & & \\
\hline No sexual experience with same-sex partner ${ }^{A}$ & 9,940 & 93.1 & $69.2 \%$ & $30.8 \%$ & & & & $\mathrm{~B}$ \\
\hline Sexual experience with same-sex partner ${ }^{B}$ & 732 & 6.9 & $76.1 \%$ & $23.9 \%$ & & & & $\mathrm{~A}$ \\
\hline Sexual Attraction & & & & & & & & \\
\hline Only attracted to opposite sex ${ }^{A}$ & 9859 & 92.4 & $69.4 \%$ & $30.6 \%$ & & & & $\mathrm{D}, \mathrm{E}$ \\
\hline Mostly attracted to opposite $\operatorname{sex}^{B}$ & 424 & 4.0 & $66.5 \%$ & $33.5 \%$ & & & & $\mathrm{D}, \mathrm{E}$ \\
\hline Equally attracted to males $\&$ females ${ }^{C}$ & 85 & 0.8 & $75.3 \%$ & $24.7 \%$ & & & & $\mathrm{D}$ \\
\hline Mostly attracted to same sex & 103 & 1.0 & $89.3 \%$ & $10.7 \%$ & & & & $\mathrm{~A}, \mathrm{~B}, \mathrm{C}, \mathrm{E}$ \\
\hline Only attracted to same sex ${ }^{E}$ & 201 & 1.9 & $77.6 \%$ & $22.4 \%$ & & & & $\mathrm{~A}, \mathrm{~B}, \mathrm{D}$ \\
\hline Age Group & & & & & & & & \\
\hline $25-34^{A}$ & 5,922 & 55.5 & $65.0 \%$ & $35.0 \%$ & & & & $\mathrm{~B}$ \\
\hline $35-44^{B}$ & 4,750 & 44.5 & $75.6 \%$ & $24.4 \%$ & & & & A \\
\hline
\end{tabular}

Note. Table presents unweighted data analyses. Row totals may not equal 100.0 due to rounding.

This sample does not include those missing values listwise on these and other variables explored in later regression analyses.

*Items in same greyscale were grouped together in above Pearson $\chi^{2}$ tests due to small cell sizes and prior examples in the literature.

Superscripts denote a Pearson $\chi^{2}$ test of $p<0.05$ between that category and its corresponding superscript. Fisher's exact two-tailed test also run for $2 \times 2$ tables; results are consistent unless marked ** (Pearson $\chi^{2}$ test of $p<0.05$; Fisher's exact two-tailed test $p>0.05$ ). 
TABLE 2-L. CH. 2 REGRESSIONS: IDENTITY: MALE

Table 2-L. Regressions of Health Outcomes on Sexual Identity for Male Respondents Age 25-45 (N=10,672)

\begin{tabular}{|c|c|c|c|c|c|c|c|c|}
\hline & \multicolumn{2}{|c|}{ Self-Rated Health } & \multicolumn{2}{|c|}{ Overweight } & \multicolumn{2}{|c|}{ Obese } & \multicolumn{2}{|c|}{ Usual Source of Care } \\
\hline & OR & $95 \%$ CI & RRR & $95 \%$ CI & RRR & $95 \%$ CI & OR & $95 \% \mathrm{CI}$ \\
\hline Intercept & $19.05 * * *$ & $(11.00,32.97)$ & 1.12 & $(0.79,1.59)$ & 0.81 & $(0.58,1.12)$ & 1.26 & $(0.89,1.77)$ \\
\hline \multicolumn{9}{|l|}{ Sexual Identity } \\
\hline Heterosexual (ref) & 1.0 & & 1.0 & & 1.0 & & 1.0 & \\
\hline Homosexual, gay, or lesbian & 1.10 & $(0.61,2.01)$ & $0.47 * *$ & $(0.31,0.73)$ & $0.63^{*}$ & $(0.42,0.95)$ & $2.26^{* * *}$ & $(1.44,3.55)$ \\
\hline Bisexual & 0.76 & $(0.41,1.40)$ & 0.89 & $(0.50,1.56)$ & 1.38 & $(0.81,2.34)$ & $1.64^{*}$ & $(1.09,2.47)$ \\
\hline \multicolumn{9}{|l|}{ Survey Cycle } \\
\hline 2011-2013 (ref) & 1.0 & & 1.0 & & 1.0 & & 1.0 & \\
\hline 2013-2015 & $0.71 *$ & $(0.53,0.96)$ & 0.83 & $(0.67,1.03)$ & 0.86 & $(0.71,1.06)$ & 0.91 & $(0.74,1.13)$ \\
\hline 2015-2017 & 0.98 & $(0.70,1.36)$ & 0.82 & $(067,1.01)$ & 0.84 & $(0.67,1.06)$ & 1.07 & $(0.86,1.32)$ \\
\hline 2017-2019 & 0.80 & $(0.59,1.09)$ & 0.88 & $(0.73,1.06)$ & 0.90 & $(0.71,1.13)$ & 0.86 & $(0.69,1.06)$ \\
\hline \multicolumn{9}{|l|}{ Age } \\
\hline 25-34 (ref) & 1.0 & & 1.0 & & 1.0 & & 1.0 & \\
\hline $35-44$ & $0.57 * * *$ & $(0.45,0.72)$ & $1.54 * * *$ & $(1.33,1.78)$ & $1.77 * * *$ & $(1.52,2.05)$ & $1.65^{* * *}$ & $(1.44,1.88)$ \\
\hline \multicolumn{9}{|l|}{ Race/ethnicity } \\
\hline White, non-Hispanic (ref) & 1.0 & & 1.0 & & 1.0 & & 1.0 & \\
\hline Hispanic & $0.73^{*}$ & $(0.54,0.98)$ & $1.37 * *$ & $(1.10,1.71)$ & $2.04 * * *$ & $(1.65,2.54)$ & 0.98 & $(0.81,1.18)$ \\
\hline Black, non-Hispanic & 0.80 & $(0.55,1.16)$ & 0.83 & $(0.68,1.02)$ & $1.43^{* *}$ & $(1.15,1.78)$ & $1.35^{* *}$ & $(1.08,1.68)$ \\
\hline Other, non-Hispanic & 0.79 & $(0.53,1.18)$ & $0.61 * * *$ & $(0.49,0.75)$ & $0.77 *$ & $(0.60,0.99)$ & $1.46^{* *}$ & $(1.16,1.84)$ \\
\hline \multicolumn{9}{|l|}{ Nativity } \\
\hline US-born (ref) & 1.0 & & 1.0 & & 1.0 & & 1.0 & \\
\hline Foreign-born & $1.51 * *$ & $(1.12,2.05)$ & 0.85 & $(0.69,1.05)$ & $0.49 * * *$ & $(0.39,0.61)$ & 0.87 & $(0.72,1.06)$ \\
\hline \multicolumn{9}{|l|}{ Residence } \\
\hline Principal MSA city (ref) & 1.0 & & 1.0 & & 1.0 & & 1.0 & \\
\hline Other MSA & 1.05 & $(0.84,1.32)$ & $1.26 * *$ & $(1.10,1.46)$ & $1.26^{* *}$ & $(1.09,1.46)$ & 1.14 & $(0.99,1.32)$ \\
\hline Not MSA & 0.91 & $(0.65,1.27)$ & 1.12 & $(0.93,1.36)$ & $1.38 * *$ & $(1.10,1.74)$ & $1.46^{* *}$ & $(1.13,1.88)$ \\
\hline \multicolumn{9}{|l|}{ Education } \\
\hline Some high school or less (ref) & 1.0 & & 1.0 & & 1.0 & & 1.0 & \\
\hline High school diploma or GED & $1.61 * *$ & $(1.19,2.17)$ & 1.12 & $(0.88,1.43)$ & $1.31^{*}$ & $(1.00,1.70)$ & 1.09 & $(0.88,1.34)$ \\
\hline Some college & $2.03 * * *$ & $(1.37,3.00)$ & 1.22 & $(0.95,1.58)$ & $1.51^{* *}$ & $(1.15,1.98)$ & $1.45^{* *}$ & $(1.13,1.88)$ \\
\hline College or graduate degree & $2.96 * * *$ & $(2.03,4.32)$ & 1.03 & $(0.80,1.33)$ & 0.98 & $(0.73,1.30)$ & $1.68 * * *$ & $(1.30,2.16)$ \\
\hline \multicolumn{9}{|l|}{ Insurance } \\
\hline Private or Medi-Gap (ref) & 1.0 & & 1.0 & & 1.0 & & 1.0 & \\
\hline Medicaid, CHIP, State-sponsored & $0.40 * * *$ & $(0.29,0.56)$ & 0.87 & $(0.67,1.12)$ & 0.83 & $(0.63,1.08)$ & 1.16 & $(0.91,1.48)$ \\
\hline Medicare, military, government & $0.36^{* * *}$ & $(0.25,0.54)$ & 1.05 & $(0.80,1.36)$ & 0.74 & $(0.54,1.01)$ & $1.71^{* *}$ & $(1.19,2.46)$ \\
\hline Single-service, Indian Health Service, not covered & $0.58^{* *}$ & $(0.43,0.79)$ & $0.78 * *$ & $(0.66,0.93)$ & $0.74 * *$ & $(0.62,0.89)$ & $0.27 * * *$ & $(0.23,0.32)$ \\
\hline \multicolumn{9}{|l|}{ Household income, \% of federal poverty level } \\
\hline $0-99$ (ref) & 1.0 & & 1.0 & & 1.0 & & 1.0 & \\
\hline $100-299$ & $1.72 * * *$ & $(1.29,2.29)$ & 1.11 & $(0.87,1.43)$ & 1.13 & $(0.90,1.41)$ & 1.21 & $(0.99,1.48)$ \\
\hline$>\mathbf{3 0 0} \%$ & $2.03 * * *$ & $(1.43,2.87)$ & 1.24 & $(0.97,1.59)$ & 0.99 & $(0.78,1.25)$ & $1.35^{* *}$ & $(1.08,1.69)$ \\
\hline \multicolumn{9}{|l|}{ Access to Usual Source of Care } \\
\hline Yes (ref) & 1.0 & & 1.0 & & 1.0 & & & \\
\hline No & $0.57 * * *$ & $(0.45,0.72)$ & 0.96 & $(0.84,1.11)$ & $0.76^{* *}$ & $(0.65,0.90)$ & & \\
\hline \multicolumn{9}{|l|}{ BMI } \\
\hline 18.5-24.9 (Normal) (ref) & 1.0 & & & & & & 1.0 & \\
\hline$<18.5$ (Underweight) & 1.18 & $(0.41,3.35)$ & & & & & 0.75 & $(0.37,1.53)$ \\
\hline 25.0-29.9 (Overweight) & 1.00 & $(0.74,1.34)$ & & & & & 1.03 & $(0.89,1.19)$ \\
\hline$>30.0$ (Obese) & $0.52 * * *$ & $(0.39,0.70)$ & & & & & $1.30 * *$ & $(1.10,1.54)$ \\
\hline
\end{tabular}

Note. Sample does not include those missing values listwise on these variables.

Self-rated general health $=0$ ) Poor, Fair 1) Good, Very good, Excellent. Logistic regression

The base outcome for the overweight and obese models is body mass index (BMI) that is low/normal (<25); overweight BMI is 25-29, and obese BMI is $>30$. Multinomial regression.

Access to a usual source of care $=0$ ) No 1) Yes. Logistic regression.

$\mathrm{OR}=$ odds ratio $\mathrm{RRR}=$ relative risk ratio $\mathrm{CI}=$ confidence interval $\mathrm{MSA}=$ metropolitan statistical area .

The $p$-values are determined by the "Pearson $\chi^{2}$ test with the Rao and Scott $(1981,1984)$ second-order correction" or the $F$ test (the default output from the Stata svy tabulate command); ${ }^{*} p<0.05, * * p<0.01, * * * p<0.001$ 
TABLE 2-M. CH. 2 REGRESSIONS: ATTRACTION: MALE

Table 2-M. Regressions of Health Outcomes on Sexual Attraction for Male Respondents Age 25-45 (N=10,672)

\begin{tabular}{|c|c|c|c|c|c|c|c|c|}
\hline & \multicolumn{2}{|c|}{ Self-Rated Health } & \multicolumn{2}{|c|}{ Overweight } & \multicolumn{2}{|c|}{ Obese } & \multicolumn{2}{|c|}{ Usual Source of Care } \\
\hline & OR & $95 \%$ CI & RRR & $95 \%$ CI & RRR & $95 \%$ CI & OR & $95 \%$ CI \\
\hline Intercept & $18.79 * * *$ & $(10.86,32.49)$ & 1.12 & $(0.79,1.58)$ & 0.81 & $(0.58,1.12)$ & 1.28 & $(0.91,1.80)$ \\
\hline \multicolumn{9}{|l|}{ Sexual Attraction } \\
\hline Only attracted to opposite sex (ref) & 1.0 & & 1.0 & & 1.0 & & 1.0 & \\
\hline Mostly attracted to opposite sex & 1.33 & $(0.73,2.42)$ & 0.88 & $(0.62,1.25)$ & 1.09 & $(0.76,1.56)$ & 0.96 & $(0.72,1.27)$ \\
\hline Equally attracted to males \& females & 0.66 & $(0.30,1.46)$ & 0.90 & $(0.37,2.21)$ & 1.76 & $(0.71,4.33)$ & 1.30 & $(0.70,2.40)$ \\
\hline Mostly attracted to same sex & 0.73 & $(0.29,1.83)$ & $0.41 *$ & $(0.20,0.82)$ & 0.60 & $(0.34,1.04)$ & $5.37 * * *$ & $(2.39,12.08)$ \\
\hline Only attracted to same sex & 1.16 & $(0.56,2.40)$ & $0.51 * *$ & $(0.31,0.83)$ & 0.63 & $(0.37,1.06)$ & $1.76^{*}$ & $(1.04,2.97)$ \\
\hline \multicolumn{9}{|l|}{ Survey Cycle } \\
\hline 2011-2013 (ref) & 1.0 & & 1.0 & & 1.0 & & 1.0 & \\
\hline 2013-2015 & $0.71 *$ & $(0.53,0.96)$ & 0.83 & $(0.67,1.03)$ & 0.86 & $(0.70,1.06)$ & 0.92 & $(0.74,1.14)$ \\
\hline 2015-2017 & 0.98 & $(0.70,1.37)$ & 0.82 & $(0.67,1.01)$ & 0.84 & $(0.67,1.06)$ & 1.07 & $(0.86,1.32)$ \\
\hline 2017-2019 & 0.80 & $(0.59,1.09)$ & 0.88 & $(0.73,1.07)$ & 0.89 & $(0.71,1.12)$ & 0.86 & $(0.69,1.07)$ \\
\hline \multicolumn{9}{|l|}{ Age } \\
\hline 25-34 (ref) & 1.0 & & 1.0 & & 1.0 & & 1.0 & \\
\hline $35-44$ & $0.57 * * *$ & $(0.45,0.72)$ & $1.53^{* * *}$ & $(1.33,1.77)$ & $1.76^{* * *}$ & $(1.52,2.05)$ & $1.66^{* * *}$ & $(1.45,1.89)$ \\
\hline \multicolumn{9}{|l|}{ Race/ethnicity } \\
\hline White, non-Hispanic (ref) & 1.0 & & 1.0 & & 1.0 & & 1.0 & \\
\hline Hispanic & $0.73 *$ & $(0.54,0.98)$ & $1.37 * *$ & $(1.10,1.72)$ & $2.05^{* * *}$ & $(1.65,2.54)$ & 0.98 & $(0.81,1.17)$ \\
\hline Black, non-Hispanic & 0.81 & $(0.56,1.16)$ & 0.84 & $(0.69,1.02)$ & $1.44 * *$ & $(1.16,1.78)$ & $1.33^{*}$ & $(1.07,1.66)$ \\
\hline Other, non-Hispanic & 0.78 & $(0.53,1.17)$ & $0.61 * * *$ & $(0.49,0.76)$ & $0.77 *$ & $(0.60,0.99)$ & $1.46^{* *}$ & $(1.16,1.84)$ \\
\hline \multicolumn{9}{|l|}{ Nativity } \\
\hline US-born (ref) & 1.0 & & 1.0 & & 1.0 & & 1.0 & \\
\hline Foreign-born & $1.51^{* *}$ & $(1.12,2.05)$ & 0.85 & $(0.69,1.05)$ & $0.49 * * *$ & $(0.39,0.60)$ & 0.87 & $(0.72,1.06)$ \\
\hline \multicolumn{9}{|l|}{ Residence } \\
\hline Principal MSA city (ref) & 1.0 & & 1.0 & & 1.0 & & 1.0 & \\
\hline Other MSA & 1.06 & $(0.84,1.33)$ & $1.26^{* *}$ & $(1.10,1.45)$ & $1.26 * *$ & $(1.09,1.47)$ & 1.14 & $(0.99,1.32)$ \\
\hline Not MSA & 0.92 & $(0.66,1.28)$ & 1.12 & $(0.92,1.36)$ & $1.39 * *$ & $(1.10,1.74)$ & $1.45^{* *}$ & $(1.12,1.88)$ \\
\hline \multicolumn{9}{|l|}{ Education } \\
\hline Some high school or less (ref) & 1.0 & & 1.0 & & 1.0 & & 1.0 & \\
\hline High school diploma or GED & $1.62^{* *}$ & $(1.20,2.18)$ & 1.13 & $(0.89,1.44)$ & $1.31 *$ & $(1.00,1.71)$ & 1.08 & $(0.87,1.33)$ \\
\hline Some college & $2.04 * * *$ & $(1.38,3.02)$ & 1.23 & $(0.95,1.59)$ & $1.51 * *$ & $(1.15,1.98)$ & $1.44 * *$ & $(1.11,1.86)$ \\
\hline College or graduate degree & $2.97 * * *$ & $(2.03,4.34)$ & 1.04 & $(0.81,1.34)$ & 0.97 & $(0.73,1.30)$ & $1.66^{* * *}$ & $(1.29,2.14)$ \\
\hline \multicolumn{9}{|l|}{ Insurance } \\
\hline Private or Medi-Gap (ref) & 1.0 & & 1.0 & & 1.0 & & 1.0 & \\
\hline Medicaid, CHIP, State-sponsored & $0.40^{* * *}$ & $(0.29,0.56)$ & 0.87 & $(0.67,1.12)$ & 0.82 & $(0.63,1.08)$ & 1.16 & $(0.91,1.48)$ \\
\hline Medicare, military, government & $0.36^{* * *}$ & $(0.25,0.54)$ & 1.05 & $(0.80,1.36)$ & 0.74 & $(0.54,1.01)$ & $1.71^{* *}$ & $(1.19,2.46)$ \\
\hline Single-service, Indian Health Service, not covered & $0.58 * * *$ & $(0.43,0.79)$ & $0.79 * *$ & $(0.66,0.94)$ & $0.74 * *$ & $(0.62,0.89)$ & $0.27 * * *$ & $(0.23,0.32)$ \\
\hline \multicolumn{9}{|l|}{ Household income, \% of federal poverty level } \\
\hline $0-99$ (ref) & 1.0 & & 1.0 & & 1.0 & & 1.0 & \\
\hline $100-299$ & $1.71 * * *$ & $(1.28,2.28)$ & 1.12 & $(0.87,1.43)$ & 1.13 & $(0.90,1.41)$ & 1.20 & $(0.98,1.48)$ \\
\hline$>\mathbf{3 0 0} \%$ & $2.02 * * *$ & $(1.43,2.86)$ & 1.24 & $(0.96,1.59)$ & 0.99 & $(0.78,1.25)$ & $1.35^{* *}$ & $(1.08,1.69)$ \\
\hline \multicolumn{9}{|l|}{ Access to Usual Source of Care } \\
\hline Yes (ref) & 1.0 & & 1.0 & & 1.0 & & & \\
\hline No & $0.57 * * *$ & $(0.45,0.72)$ & 0.96 & $(0.84,1.11)$ & $0.76 * *$ & $(0.64,0.90)$ & & \\
\hline \multicolumn{9}{|l|}{ BMI } \\
\hline 18.5-24.9 (Normal) (ref) & 1.0 & & & & & & 1.0 & \\
\hline$<\mathbf{1 8 . 5}$ (Underweight) & 1.18 & $(0.42,3.38)$ & & & & & 0.75 & $(0.37,1.52)$ \\
\hline 25.0-29.9 (Overweight) & 1.00 & $(0.74,1.35)$ & & & & & 1.03 & $(0.89,1.19)$ \\
\hline$>\mathbf{3 0 . 0}$ (Obese) & $0.52 * * *$ & $(0.39,0.70)$ & & & & & $1.31 * *$ & $(1.10,1.55)$ \\
\hline
\end{tabular}

Note. Sample does not include those missing values listwise on these variables.

Self-rated general health $=0$ ) Poor, Fair 1) Good, Very good, Excellent. Logistic regression

The base outcome for the overweight and obese models is body mass index (BMI) that is low/normal (<25); overweight BMI is 25-29, and obese BMI is $>30$. Multinomial regression.

Access to a usual source of care $=0$ ) No 1) Yes. Logistic regression.

$\mathrm{OR}=$ odds ratio $; \mathrm{RRR}=$ relative risk ratio $\mathrm{CI}=$ confidence interval $; \mathrm{MSA}=$ metropolitan statistical area.

The $p$-values are determined by the "Pearson $\chi^{2}$ test with the Rao and Scott $(1981,1984)$ second-order correction" or the $F$ test (the default output from the Stata svy

tabulate command); ${ }^{*} p<0.05, * * p<0.01,{ }^{* * *} p<0.001$ 
TABLE 2-N. CH. 2 REGRESSIONS: BEHAVIOR: MALE

Table 2-N. Regressions of Health Outcomes on Sexual Behavior for Male Respondents Age 25-45 (N=10,672)

\begin{tabular}{|c|c|c|c|c|c|c|c|c|}
\hline & \multicolumn{2}{|c|}{ Self-Rated Health } & \multicolumn{2}{|c|}{ Overweight } & \multicolumn{2}{|c|}{ Obese } & \multicolumn{2}{|c|}{ Usual Source of Care } \\
\hline & OR & $95 \%$ CI & RRR & $95 \% \mathrm{CI}$ & RRR & $95 \% \mathrm{CI}$ & OR & $95 \%$ CI \\
\hline Intercept & $19.52 * * *$ & $(11.25,33.88)$ & 1.12 & $(0.79,1.58)$ & 0.81 & $(0.58,1.13)$ & 1.26 & $(0.89,1.78)$ \\
\hline \multicolumn{9}{|l|}{ Sexual Behavior } \\
\hline No sexual experience with same-sex partner (ref) & 1.0 & & 1.0 & & 1.0 & & 1.0 & \\
\hline Sexual experience with same-sex partner & $0.62 * *$ & $(0.43,0.89)$ & 0.77 & $(0.59,1.02)$ & 0.96 & $(0.75,1.23)$ & $1.51^{* *}$ & $(1.14,2.01)$ \\
\hline \multicolumn{9}{|l|}{ Survey Cycle } \\
\hline 2011-2013 (ref) & 1.0 & & 1.0 & & 1.0 & & 1.0 & \\
\hline 2013-2015 & $0.71 *$ & $(0.53,0.96)$ & 0.83 & $(0.67,1.03)$ & 0.87 & $(0.71,1.06)$ & 0.91 & $(0.74,1.13)$ \\
\hline 2015-2017 & 0.98 & $(0.70,1.37)$ & 0.82 & $(0.66,1.01)$ & 0.84 & $(0.66,1.05)$ & 1.07 & $(0.86,1.32)$ \\
\hline 2017-2019 & 0.81 & $(0.59,1.10)$ & 0.88 & $(0.72,1.06)$ & 0.89 & $(0.0 .71,1.12)$ & 0.86 & $(0.69,1.06)$ \\
\hline \multicolumn{9}{|l|}{ Age } \\
\hline 25-34 (ref) & 1.0 & & 1.0 & & 1.0 & & 1.0 & \\
\hline $35-44$ & $0.57 * * *$ & $(0.45,0.72)$ & $1.54 * * *$ & $(1.33,1.78)$ & $1.76^{* * *}$ & $(1.52,2.05)$ & $1.64 * * *$ & $(1.44,1.88)$ \\
\hline \multicolumn{9}{|l|}{ Race/ethnicity } \\
\hline White, non-Hispanic (ref) & 1.0 & & 1.0 & & 1.0 & & 1.0 & \\
\hline Hispanic & $0.73 *$ & $(0.54,0.98)$ & $1.36^{* *}$ & $(1.09,1.69)$ & $2.02 * * *$ & $(1.63,2.51)$ & 0.99 & $(0.82,1.19)$ \\
\hline Black, non-Hispanic & 0.78 & $(0.54,1.13)$ & 0.83 & $(0.68,1.01)$ & $1.43 * *$ & $(1.15,1.77)$ & $1.35^{* *}$ & $(1.08,1.68)$ \\
\hline Other, non-Hispanic & 0.79 & $(0.53,1.17)$ & $0.60 * * *$ & $(0.48,0.75)$ & $0.77 *$ & $(0.60,0.99)$ & $1.47 * *$ & $(1.16,1.85)$ \\
\hline \multicolumn{9}{|l|}{ Nativity } \\
\hline US-born (ref) & 1.0 & & 1.0 & & 1.0 & & 1.0 & \\
\hline Foreign-born & $1.49^{*}$ & $(1.10,2.01)$ & 0.85 & $(0.70,1.05)$ & $0.49 * * *$ & $(0.39,0.61)$ & 0.87 & $(0.72,1.06)$ \\
\hline \multicolumn{9}{|l|}{ Residence } \\
\hline Principal MSA city (ref) & 1.0 & & 1.0 & & 1.0 & & 1.0 & \\
\hline Other MSA & 1.05 & $(0.83,1.32)$ & $1.27 * *$ & $(1.10,1.46)$ & $1.26 * *$ & $(1.09,1.47)$ & 1.14 & $(0.99,1.32)$ \\
\hline Not MSA & 0.89 & $(0.64,1.24)$ & 1.12 & $(0.92,1.36)$ & $1.38 * *$ & $(1.10,1.74)$ & $1.46^{* *}$ & $(1.13,1.89)$ \\
\hline \multicolumn{9}{|l|}{ Education } \\
\hline Some high school or less (ref) & 1.0 & & 1.0 & & 1.0 & & 1.0 & \\
\hline High school diploma or GED & $1.63 * *$ & $(1.21,2.19)$ & 1.13 & $(0.89,1.43)$ & $1.31 *$ & $(1.00,1.70)$ & 1.08 & $(0.88,1.34)$ \\
\hline Some college & $2.06 * * *$ & $(1.39,3.06)$ & 1.22 & $(0.95,1.58)$ & $1.50 * *$ & $(1.15,1.97)$ & $1.45^{* *}$ & $(1.12,1.87)$ \\
\hline College or graduate degree & $3.02 * * *$ & $(2.08,4.40)$ & 1.03 & $(0.80,1.33)$ & 0.97 & $(0.73,1.29)$ & $1.67^{* * *}$ & $(1.29,2.16)$ \\
\hline \multicolumn{9}{|l|}{ Insurance } \\
\hline Private or Medi-Gap (ref) & 1.0 & & 1.0 & & 1.0 & & 1.0 & \\
\hline Medicaid, CHIP, State-sponsored & $0.41 * * *$ & $(0.29,0.57)$ & 0.86 & $(0.67,1.11)$ & 0.82 & $(0.63,1.08)$ & 1.16 & $(0.91,1.48)$ \\
\hline Medicare, military, government & $0.37 * * *$ & $(0.25,0.55)$ & 1.05 & $(0.81,1.37)$ & 0.74 & $(0.54,1.01)$ & $1.70^{* *}$ & $(1.18,2.43)$ \\
\hline Single-service, Indian Health Service, not covered & $0.59 * * *$ & $(0.43,0.79)$ & $0.78 * *$ & $(0.66,0.94)$ & $0.74 * *$ & $(0.62,0.89)$ & $0.27^{* * *}$ & $(0.23,0.32)$ \\
\hline \multicolumn{9}{|l|}{ Household income, \% of federal poverty level } \\
\hline $0-99$ (ref) & 1.0 & & 1.0 & & 1.0 & & 1.0 & \\
\hline $100-299$ & $1.71 * * *$ & $(1.28,2.28)$ & 1.11 & $(0.87,1.42)$ & 1.12 & $(0.90,1.40)$ & 1.21 & $(0.99,1.48)$ \\
\hline$>\mathbf{3 0 0} \%$ & $2.00 * * *$ & $(1.41,2.82)$ & 1.23 & $(0.96,1.58)$ & 0.98 & $(0.78,1.24)$ & $1.36^{* *}$ & $(1.09,1.70)$ \\
\hline \multicolumn{9}{|l|}{ Access to Usual Source of Care } \\
\hline Yes (ref) & 1.0 & & 1.0 & & 1.0 & & & \\
\hline No & $0.58 * * *$ & $(0.46,0.73)$ & 0.97 & $(0.84,1.12)$ & $0.77 * *$ & $(0.65,0.91)$ & & \\
\hline \multicolumn{9}{|l|}{ BMI } \\
\hline 18.5-24.9 (Normal) (ref) & 1.0 & & & & & & 1.0 & \\
\hline$<18.5$ (Underweight) & 1.15 & $(0.40,3.30)$ & & & & & 0.75 & $(0.37,1.53)$ \\
\hline 25.0-29.9 (Overweight) & 0.99 & $(0.73,1.34)$ & & & & & 1.02 & $(0.89,1.18)$ \\
\hline$>30.0$ (Obese) & $0.52 * * *$ & $(0.39,0.69)$ & & & & & $1.30 * *$ & $(1.10,1.54)$ \\
\hline
\end{tabular}

Note. Sample does not include those missing values listwise on these variables.

Self-rated general health =0) Poor, Fair 1) Good, Very good, Excellent. Logistic regression

The base outcome for the overweight and obese models is body mass index (BMI) that is low/normal ( $<25)$; overweight BMI is $25-29$, and obese BMI is $>30$. Multinomial regression.

Access to a usual source of care $=0$ ) No 1) Yes. Logistic regression.

$\mathrm{OR}=$ odds ratio; $\mathrm{RRR}=$ relative risk ratio; $\mathrm{CI}=$ confidence interval; $\mathrm{MSA}=$ metropolitan statistical area.

The $p$-values are determined by the "Pearson $\chi^{2}$ test with the Rao and Scott $(1981,1984)$ second-order correction" or the $F$ test (the default output from the Stata svy

tabulate command); ${ }^{*} p<0.05,{ }^{* *} p<0.01,{ }^{* * *} p<0.001$ 
Table 3-A. Characteristics of US Adults Aged 25-34, by Sexual Identity 2011-2019 (N=13,038)

\begin{tabular}{|c|c|c|c|c|c|c|}
\hline & \multicolumn{6}{|c|}{ Sexual Identity } \\
\hline & \multicolumn{2}{|c|}{$\begin{array}{l}\text { Heterosexual or straight } \\
\quad(\mathrm{n}=12,056 ; 92.5 \%)^{\mathrm{a}}\end{array}$} & \multicolumn{2}{|c|}{$\begin{array}{c}\text { Homosexual, gay, or } \\
\text { lesbian } \\
(\mathrm{n}=306 ; 2.3 \%)^{\mathrm{a}}\end{array}$} & \multicolumn{2}{|c|}{$\begin{array}{c}\text { Bisexual } \\
(\mathrm{n}=676 ; 5.2 \%)^{\mathrm{a}}\end{array}$} \\
\hline & Mean \% & $95 \% \mathrm{CI}$ & Mean \% & $95 \%$ CI & Mean \% & $95 \% \mathrm{CI}$ \\
\hline \multicolumn{7}{|l|}{ Sexual Attraction*** } \\
\hline Only attracted to opposite sex & 90.4 & $(89.5,91.2)$ & 2.0 & $(0.6,6.3)$ & 4.9 & $(2.9,8.2)$ \\
\hline Mostly attracted to opposite sex & 8.9 & $(8.1,9.8)$ & 0.5 & $(0.1,1.6)$ & 41.1 & $(35.3,47.1)$ \\
\hline Equally attracted to males \& females & 0.6 & $(0.4,0.8)$ & 1.9 & $(0.9,4.0)$ & 47.0 & $(41.5,52.7)$ \\
\hline Mostly attracted to same sex & 0.0 & $(0.0,0.0)$ & 28.0 & $(20.1,37.4)$ & 6.6 & $(4.4,9.8)$ \\
\hline Only attracted to same sex & 0.1 & $(0.1,0.2)$ & 67.6 & $(58.5,75.6)$ & 0.4 & $(0.1,1.4)$ \\
\hline \multicolumn{7}{|l|}{ Sexual Behavior**** } \\
\hline Sexual experience with same-sex partner & 8.0 & $(7.3,8.7)$ & 96.2 & $(91.9,98.3)$ & 80.5 & $(74.8,85.2)$ \\
\hline No sexual experience with same-sex partner & 92.0 & $(91.3,92.7)$ & 3.8 & $(1.7,8.1)$ & 19.5 & $(14.8,25.2)$ \\
\hline \multicolumn{7}{|l|}{ Sex $* * *$} \\
\hline Female & 46.8 & $(45.5,48.2)$ & 51.2 & $(42.0,60.2)$ & 78.1 & $(72.8,82.6)$ \\
\hline Male & 53.2 & $(51.8,54.5)$ & 48.8 & $(39.8,58.0)$ & 21.9 & $(17.4,27.2)$ \\
\hline \multicolumn{7}{|l|}{ Race/ethnicity* } \\
\hline White, non-Hispanic & 57.2 & $(54.9,59.6)$ & 46.6 & $(37.5,56.0)$ & 63.5 & $(58.0,68.7)$ \\
\hline Hispanic & 20.0 & $(18.1,22.1)$ & 25.7 & $(17.5,36.1)$ & 14.7 & $(11.6,18.3)$ \\
\hline Black, non-Hispanic & 12.5 & $(11.1,14.0)$ & 13.3 & $(8.6,20.1)$ & 12.4 & $(9.6,15.8)$ \\
\hline Other, non-Hispanic & 10.3 & $(9.1,11.6)$ & 14.3 & $(9.4,21.3)$ & 9.4 & $(6.4,13.7)$ \\
\hline \multicolumn{7}{|l|}{ Residence } \\
\hline Principal MSA city & 36.2 & $(33.0,39.6)$ & 44.4 & $(36.3,52.8)$ & 38.7 & $(33.1,44.6)$ \\
\hline Other MSA & 48.4 & $(45.0,51.8)$ & 45.8 & $(37.2,54.6)$ & 47.1 & $(41.3,53.0)$ \\
\hline Not MSA & 15.4 & $(12.5,18.9)$ & 9.8 & $(5.4,17.2)$ & 14.2 & $(9.9,19.9)$ \\
\hline \multicolumn{7}{|l|}{ Nativity** } \\
\hline US-born & 83.1 & $(81.8,84.4)$ & 86.9 & $(78.4,92.3)$ & 91.6 & $(88.1,94.2)$ \\
\hline Foreign-born & 16.9 & $(15.6,18.2)$ & 13.1 & $(7.7,21.6)$ & 8.4 & $(5.8,11.9)$ \\
\hline \multicolumn{7}{|l|}{ Education } \\
\hline Some high school or less & 9.8 & $(8.8,10.8)$ & 5.5 & $(3.2,9.5)$ & 8.9 & $(6.3,12.5)$ \\
\hline High school diploma or GED & 25.9 & $(24.4,27.4)$ & 26.5 & $(19.3,35.2)$ & 26.1 & $(21.5,31.4)$ \\
\hline Some college & 20.8 & $(19.7,22.0)$ & 25.1 & $(19.0,32.3)$ & 22.7 & $(18.5,27.5)$ \\
\hline College or graduate degree & 43.5 & $(41.4,45.8)$ & 42.9 & $(34.9,51.4)$ & 42.2 & $(36.4,48.4)$ \\
\hline \multicolumn{7}{|l|}{ Insurance $* * *$} \\
\hline Private or Medi-Gap & 60.0 & $(58.1,61.9)$ & 62.1 & $(53.6,70.0)$ & 52.8 & $(47.3,58.1)$ \\
\hline Medicaid, CHIP, State-sponsored & 13.7 & $(12.6,14.9)$ & 11.5 & $(8.1,16.1)$ & 22.9 & $(18.7,27.8)$ \\
\hline Medicare, military, government & 5.1 & $(4.2,6.2)$ & 6.0 & $(3.4,10.3)$ & 4.2 & $(2.9,6.0)$ \\
\hline Single-service, Indian Health Service, not covered & 21.2 & $(19.7,22.7)$ & 20.3 & $(14.1,28.4)$ & 20.1 & $(16.2,24.7)$ \\
\hline \multicolumn{7}{|l|}{ Household income, \% of federal poverty level** } \\
\hline $0-99$ & 19.0 & $(17.9,20.2)$ & 21.5 & $(14.9,30.0)$ & 24.2 & $(20.2,28.6)$ \\
\hline 100-299 & 39.2 & $(37.6,40.8)$ & 37.0 & $(29.2,45.4)$ & 45.9 & $(40.2,51.6)$ \\
\hline$>300 \%$ & 41.8 & $(39.9,43.7)$ & 41.5 & $(32.5,51.2)$ & 30.0 & $(24.8,35.7)$ \\
\hline \multicolumn{7}{|l|}{ Self-rated health $* * *$} \\
\hline Poor & 0.9 & $(0.6,1.1)$ & 3.8 & $(1.1,12.6)$ & 1.5 & $(0.9,2.6)$ \\
\hline Fair & 5.0 & $(4.4,5.6)$ & 4.6 & $(2.6,7.9)$ & 9.6 & $(7.4,12.5)$ \\
\hline Good & 24.4 & $(23.2,25.5)$ & 22.6 & $(17.0,29.4)$ & 33.1 & $(27.8,38.8)$ \\
\hline Very good & 39.7 & $(38.4,41.0)$ & 34.4 & $(27.3,42.3)$ & 35.0 & $(29.8,40.6)$ \\
\hline Excellent & 30.2 & $(28.9,31.5)$ & 34.6 & $(26.7,43.5)$ & 20.8 & $(16.8,25.5)$ \\
\hline \multicolumn{7}{|l|}{ BMI* } \\
\hline$<18.5$ (Underweight) & 1.4 & $(1.1,1.8)$ & 1.0 & $(0.4,2.7)$ & 0.8 & $(0.4,1.6)$ \\
\hline 18.5-24.9 (Normal) & 36.7 & $(35.3,38.1)$ & 41.5 & $(33.6,49.9)$ & 34.2 & $(28.9,39.9)$ \\
\hline 25.0-29.9 (Overweight) & 32.7 & $(31.4,34.1)$ & 28.8 & $(21.8,37.0)$ & 27.9 & $(23.2,33.3)$ \\
\hline$>30.0$ (Obese) & 29.2 & $(27.9,30.5)$ & 28.7 & $(22.6,35.6)$ & 37.1 & $(31.7,42.9)$ \\
\hline Access to Usual Source of Care* & & & & & & \\
\hline Yes & 74.3 & $(72.9,75.6)$ & 82.1 & $(75.3,87.4)$ & 78.6 & $(73.9,82.6)$ \\
\hline No & 25.7 & $(24.4,27.1)$ & 17.9 & $(12.6,24.7)$ & 21.4 & $(17.4,26.1)$ \\
\hline Cycle $* * *$ & & & & & & \\
\hline 2011-2013 & 25.5 & $(23.1,28.0)$ & 16.8 & $(11.3,24.2)$ & 20.4 & $(16.3,25.3)$ \\
\hline 2013-2015 & 26.9 & $(24.5,29.4)$ & 21.4 & $(15.5,28.8)$ & 20.1 & $(16.0,24.9)$ \\
\hline 2015-2017 & 23.5 & $(21.1,26.1)$ & 33.9 & $(24.2,45.1)$ & 24.7 & $(20.2,29.8)$ \\
\hline 2017-2019 & 24.1 & $(21.7,26.6)$ & 27.9 & $(20.9,36.3)$ & 34.8 & $(29.4,40.6)$ \\
\hline
\end{tabular}

${ }^{a} \mathrm{n}=$ sample number and percent without using svy command; Mean and $95 \%$ CI were conducted using svy command.

${ }^{*} \mathrm{p}<0.05,{ }^{* *} \mathrm{p}<0.01,{ }^{* * *} \mathrm{p}<0.001 ; p$-values were determined using the "Pearson $\chi 2$ test with the Rao and Scott $(1981,1984)$ second-order correction" or the $F$ test (the default output from the Stata Svy tabulate command) (Stata.com n.d.:11).

Note. Sample does not include pregnant females or those missing values listwise on these variables. MSA = metropolitan statistical area; $\mathrm{BMI}=$ body mass index; not all strata included in each cell if missing subpopulation members. 
TABLE 3-B. CH. 3 SUMMARY STATISTICS: ATTRACTION: 25-34

Table 3-B. Characteristics of US Adults Aged 25-34, by Sexual Attraction 2011-2019 (N=13,038)

\begin{tabular}{|c|c|c|c|c|c|c|c|c|c|c|}
\hline & \multicolumn{10}{|c|}{ Sexual Attraction } \\
\hline & \multicolumn{2}{|c|}{$\begin{array}{c}\text { Only attracted to } \\
\text { opposite sex } \\
(\mathrm{n}=10,885 ; 83.5 \%)^{\mathrm{a}}\end{array}$} & \multicolumn{2}{|c|}{$\begin{array}{c}\text { Mostly attracted to } \\
\text { opposite sex } \\
(\mathrm{n}=1,350 ; 10.4 \%)^{a}\end{array}$} & \multicolumn{2}{|c|}{$\begin{array}{c}\text { Equally attracted to } \\
\text { males and females } \\
(\mathrm{n}=444 ; 3.4 \%)^{\mathrm{a}}\end{array}$} & \multicolumn{2}{|c|}{$\begin{array}{c}\text { Mostly attracted to } \\
\text { same sex } \\
(\mathrm{n}=138 ; 1.1 \%)^{\mathrm{a}}\end{array}$} & \multicolumn{2}{|c|}{$\begin{array}{c}\text { Only attracted to } \\
\text { same sex } \\
(n=221 ; 1.7 \%)^{a}\end{array}$} \\
\hline & Mean \% & $95 \%$ CI & Mean \% & $95 \%$ CI & Mean \% & $95 \%$ CI & Mean \% & $95 \%$ CI & Mean \% & $95 \%$ CI \\
\hline \multicolumn{11}{|l|}{ Sexual Identity*** } \\
\hline Heterosexual & 99.7 & $(99.5,99.8)$ & 82.1 & $(79.0,84.7)$ & 20.2 & $(14.7,27.1)$ & 1.3 & $(0.3,4.6)$ & 5.9 & $(3.5,9.9)$ \\
\hline Homosexual, gay, or lesbian & 0.1 & $(0.0,0.2)$ & 0.1 & $(0.0,0.4)$ & 1.6 & $(0.8,3.4)$ & 67.8 & $(54.6,78.7)$ & 93.1 & $(89.0,95.7)$ \\
\hline Bisexual & 0.3 & $(0.1,0.4)$ & 17.8 & $(15.2,20.9)$ & 78.2 & $(71.3,83.8)$ & 30.9 & $(20.3,44.0)$ & 1.0 & $(0.3,3.5)$ \\
\hline \multicolumn{11}{|l|}{ Sexual Behavior*** } \\
\hline Sexual experience with same-sex partner & 4.9 & $(4.4,5.5)$ & 45.2 & $(41.4,49.1)$ & 76.3 & $(69.5,82.1)$ & 96.0 & $(89.0,98.6)$ & 91.9 & $(87.6,94.8)$ \\
\hline No sexual experience with same-sex partner & 95.1 & $(94.5,95.6)$ & 54.8 & $(50.9,58.6)$ & 23.7 & $(17.9,30.5)$ & 4.0 & $(1.4,11.0)$ & 8.1 & $(5.2,12.4)$ \\
\hline \multicolumn{11}{|l|}{ Sex $* * *$} \\
\hline Female & 43.4 & $(42.0,44.8)$ & 79.9 & $(76.7,82.6)$ & 83.1 & $(74.8,89.1)$ & 40.3 & $(27.8,54.2)$ & 54.9 & $(44.5,64.9)$ \\
\hline Male & 56.6 & $(55.2,58.0)$ & 20.1 & $(17.4,23.3)$ & 16.9 & $(10.9,25.2)$ & 59.7 & $(45.8,72.2)$ & 45.1 & $(35.1,55.5)$ \\
\hline \multicolumn{11}{|l|}{ Race/ethnicity*** } \\
\hline White, non-Hispanic & 56.3 & $(53.9,58.7)$ & 66.2 & $(62.4,69.9)$ & 63.2 & $(57.4,68.7)$ & 43.2 & $(29.5,58.1)$ & 51.1 & $(41.2,60.8)$ \\
\hline Hispanic & 20.5 & $(18.5,22.7)$ & 15.1 & $(12.7,17.8)$ & 13.3 & $(9.8,17.7)$ & 30.0 & $(15.8,49.4)$ & 21.5 & $(14.7,30.2)$ \\
\hline Black, non-Hispanic & 12.8 & $(11.3,14.4)$ & 9.1 & $(7.0,11.7)$ & 13.4 & $(10.1,17.5)$ & 13.8 & $(8.1,22.7)$ & 14.7 & $(9.3,22.4)$ \\
\hline Other, non-Hispanic & 10.4 & $(9.1,11.8)$ & 9.5 & $(7.4,12.3)$ & 10.1 & $(6.8,14.7)$ & 12.9 & $(7.8,20.7)$ & 12.8 & $(7.3,21.4)$ \\
\hline \multicolumn{11}{|l|}{ Residence } \\
\hline Principal MSA city & 35.7 & $(32.4,39.1)$ & 40.7 & $(35.8,45.7)$ & 38.6 & $(31.6,46.1)$ & 50.8 & $(41.1,60.5)$ & 40.8 & $(31.5,50.7)$ \\
\hline Other MSA & 48.6 & $(45.2,52.1)$ & 46.0 & $(41.2,50.8)$ & 48.5 & $(41.3,55.8)$ & 39.7 & $(30.1,50.1)$ & 47.3 & $(37.4,57.4)$ \\
\hline Not MSA & 15.7 & $(12.7,19.2)$ & 13.4 & $(9.5,18.5)$ & 12.9 & $(9.0,18.1)$ & 9.5 & $(4.3,19.4)$ & 12.0 & $(6.4,21.4)$ \\
\hline \multicolumn{11}{|l|}{ Nativity*** } \\
\hline US-born & 82.4 & $(81.0,83.8)$ & 90.8 & $(88.4,92.7)$ & 87.3 & $(80.9,91.8)$ & 88.9 & $(79.7,94.2)$ & 88.6 & $(77.8,94.5)$ \\
\hline Foreign-born & 17.6 & $(16.2,19.0)$ & 9.2 & $(7.3,11.6)$ & 12.7 & $(8.2,19.1)$ & 11.1 & $(5.8,20.3)$ & 11.4 & $(5.5,22.2)$ \\
\hline \multicolumn{11}{|l|}{ Education $* * *$} \\
\hline Some high school or less & 10.4 & $(9.4,11.5)$ & 5.2 & $(3.9,6.9)$ & 6.8 & $(4.4,10.3)$ & 2.5 & $(1.0,6.1)$ & 7.3 & $(4.2,12.4)$ \\
\hline High school diploma or GED & 26.7 & $(25.0,28.3)$ & 20.3 & $(16.8,24.4)$ & 23.6 & $(19.1,28.7)$ & 18.9 & $(11.7,29.2)$ & 28.2 & $(19.9,38.2)$ \\
\hline Some college & 20.5 & $(19.4,21.7)$ & 22.5 & $(19.4,25.9)$ & 26.3 & $(20.9,32.5)$ & 27.5 & $(18.5,39.0)$ & 23.5 & $(17.2,31.2)$ \\
\hline College or graduate degree & 42.4 & $(40.2,44.7)$ & 52.0 & $(47.6,56.3)$ & 43.4 & $(36.6,50.4)$ & 51.0 & $(41.3,60.6)$ & 41.0 & $(31.7,51.0)$ \\
\hline \multicolumn{11}{|l|}{ Insurance* $*$} \\
\hline Private or Medi-Gap & 59.8 & $(57.8,61.7)$ & 60.8 & $(56.7,64.8)$ & 53.4 & $(46.9,59.8)$ & 61.3 & $(48.3,72.8)$ & 61.1 & $(51.6,69.8)$ \\
\hline Medicaid, CHIP, State-sponsored & 13.6 & $(12.5,14.8)$ & 16.2 & $(13.5,19.4)$ & 22.3 & $(18.0,27.3)$ & 11.3 & $(7.2,17.2)$ & 12.7 & $(8.4,18.8)$ \\
\hline Medicare, military, government & 5.1 & $(4.2,6.2)$ & 5.1 & $(3.6,7.0)$ & 4.6 & $(3 \cdot 0,7.1)$ & 5.0 & $(2.3,10.4)$ & 6.1 & $(2.9,12.2)$ \\
\hline $\begin{array}{l}\text { Single-service, Indian Health Service, not } \\
\text { covered }\end{array}$ & 21.5 & $(20.0,23.1)$ & 17.9 & $(14.9,21.3)$ & 19.6 & $(14.8,25.6)$ & 22.5 & $(14.1,34.0)$ & 20.1 & $(13.4,29.0)$ \\
\hline Household income, $\%$ of federal poverty level & & & & & & & & & & \\
\hline 0-99 & 19.2 & $(18.1,20.5)$ & 16.8 & $(14.0,20.0)$ & 27.2 & $(21.1,34.4)$ & 20.4 & $(12.4,31.7)$ & 23.4 & $(15.7,33.3)$ \\
\hline 100-299 & 39.3 & $(37.6,41.0)$ & 41.3 & $(37.3,45.4)$ & 39.3 & $(33.2,45.8)$ & 35.0 & $(23.8,48.2)$ & 40.5 & $(31.0,50.6)$ \\
\hline$>\mathbf{3 0 0} \%$ & 41.5 & $(39.6,43.4)$ & 41.9 & $(37.9,46.0)$ & 33.5 & $(26.6,41.1)$ & 44.6 & $(30.1,60.1)$ & 36.2 & $(26.4,47.3)$ \\
\hline Self-rated health $* * *$ & & & & & & & & & & \\
\hline Poor & 0.8 & $(0.6,1.1)$ & 1.1 & $(0.6,2.3)$ & 1.7 & $(0.9,3.6)$ & 2.3 & $(0.6,8.2)$ & 4.2 & $(0.9,17.4)$ \\
\hline Fair & 4.9 & $(4.4,5.6)$ & 5.9 & $(4.4,7.8)$ & 9.6 & $(7.3,12.6)$ & 6.2 & $(3.0,12.5)$ & 4.1 & $(2.1,8.2)$ \\
\hline Good & 24.0 & $(22.8,25.2)$ & 30.1 & $(26.4,34.0)$ & 27.5 & $(22.1,33.6)$ & 27.9 & $(18.2,40.2)$ & 22.2 & $(16.1,29.8)$ \\
\hline Very good & 39.3 & $(38.0,40.7)$ & 40.7 & $(36.7,44.8)$ & 37.5 & $(31.0,44.4)$ & 40.0 & $(30.1,50.7)$ & 33.6 & $(25.6,42.6)$ \\
\hline Excellent & 30.9 & $(29.5,32.3)$ & 22.3 & $(19.3,25.5)$ & 23.7 & $(18.0,30.5)$ & 23.6 & $(14.8,35.4)$ & 35.9 & $(25.7,47.6)$ \\
\hline BMI* & & & & & & & & & & \\
\hline$<18.5$ (Underweight) & 1.4 & $(1.1,1.8)$ & 1.4 & $(0.7,2.7)$ & 1.4 & $(0.6,2.9)$ & 0.5 & $(0.1,3.5)$ & 1.1 & $(0.4,3.4)$ \\
\hline 18.5-24.9 (Normal) & 36.0 & $(34.5,37.5)$ & 41.5 & $(37.9,45.1)$ & 36.8 & $(29.7,44.4)$ & 35.9 & $(26.8,46.2)$ & 42.5 & $(32.4,53.3)$ \\
\hline 25.0-29.9 (Overweight) & 33.4 & $(32.0,34.9)$ & 26.8 & $(23.5,30.5)$ & 24.9 & $(19.8,30.9)$ & 30.9 & $(20.1,44.2)$ & 29.1 & $(20.8,38.9)$ \\
\hline$>\mathbf{3 0 . 0}$ (Obese) & 29.2 & $(27.8,30.6)$ & 30.3 & $(26.5,34.3)$ & 37.0 & $(30.6,43.7)$ & 32.7 & $(23.5,43.4)$ & 27.3 & $(20.1,35.9)$ \\
\hline Access to Usual Source of Care** & & & & & & & & & & \\
\hline Yes & 73.8 & $(72.4,75.2)$ & 78.8 & $(75.4,81.8)$ & 77.7 & $(71.9,82.6)$ & 88.9 & $(79.0,94.5)$ & 78.4 & $(70.1,84.8)$ \\
\hline No & 26.2 & $(24.8,27.6)$ & 21.2 & $(18.2,24.6)$ & 22.3 & $(17.4,28.1)$ & 11.1 & $(5.5,21.0)$ & 21.6 & $(15.2,29.9)$ \\
\hline Cycle*** & & & & & & & & & & \\
\hline 2011-2013 & 25.9 & $(23.5,28.4)$ & 21.6 & $(17.9,25.9)$ & 20.5 & $(15.8,26.1)$ & 21.3 & $(12.5,33.9)$ & 16.2 & $(10.4,24.3)$ \\
\hline 2013-2015 & 27.0 & $(24.5,29.6)$ & 23.1 & $(19.2,27.6)$ & 27.5 & $(21.3,34.6)$ & 19.4 & $(11.6,30.8)$ & 22.8 & $(16.0,31.4)$ \\
\hline 2015-2017 & 23.6 & $(21.2,26.1)$ & 24.1 & $(19.8,29.0)$ & 20.9 & $(16.6,26.1)$ & 36.1 & $(20.9,54.6)$ & 32.1 & $(22.9,42.8)$ \\
\hline 2017-2019 & 23.6 & $(21.3,26.0)$ & 31.1 & $(25.4,37.4)$ & 31.1 & $(25.1,37.8)$ & 23.2 & $(14.7,34.7)$ & 28.9 & $(21.3,38.0)$ \\
\hline
\end{tabular}

${ }^{a} \mathrm{n}=$ sample number and percent without using svy command; Mean and $95 \%$ CI were conducted using svy command.

${ }^{*} \mathrm{p}<0.05, * * \mathrm{p}<0.01,{ }^{* * *} \mathrm{p}<0.001 ; p$-values were determined using the "Pearson $\chi 2$ test with the Rao and Scott $(1981,1984)$ second-order correction" or the $F$ test (the default output from the Stata svy tabulate command) (Stata.com n.d.:11).

Note. Sample does not include pregnant females or those missing values listwise on these variables. MSA = metropolitan statistical area; BMI = body mass index; not all strata included in each cell if missing subpopulation members. 
Table 3-C. Characteristics of US Adults Aged 25-34, by Sexual Behavior 2011-2019 (N=13,038)

\begin{tabular}{|c|c|c|c|c|}
\hline & \multicolumn{4}{|c|}{$\begin{array}{l}\text { Sexual Behavior } \\
\end{array}$} \\
\hline & \multicolumn{2}{|c|}{$\begin{array}{c}\text { Sexual experience } \\
\text { with same sex } \\
\text { partner } \\
(\mathrm{n}=1,955 ; 15.0 \%)^{\mathrm{a}}\end{array}$} & \multicolumn{2}{|c|}{$\begin{array}{c}\text { No sexual experience } \\
\text { with same sex } \\
\text { partner } \\
(n=11,083 ; 85.0 \%)^{a}\end{array}$} \\
\hline & Mean \% & $95 \% \mathrm{CI}$ & Mean \% & $95 \%$ CI \\
\hline \multicolumn{5}{|l|}{ Sexual Identity**** } \\
\hline Heterosexual & 56.4 & $(53.1,59.7)$ & 98.9 & $(98.6,99.2)$ \\
\hline Homosexual, gay, or lesbian & 16.7 & $(14.0,19.7)$ & 0.1 & $(0.0,0.2)$ \\
\hline Bisexual & 26.9 & $(24.3,29.7)$ & 1.0 & $(0.7,1.3)$ \\
\hline \multicolumn{5}{|l|}{ Sexual Attraction $* * *$} \\
\hline Only attracted to opposite sex & 31.5 & $(28.4,34.7)$ & 92.7 & $(91.8,93.4)$ \\
\hline Mostly attracted to opposite sex & 34.8 & $(31.6,38.1)$ & 6.4 & $(5.7,7.2)$ \\
\hline Equally attracted to males \& females & 15.3 & $(13.4,17.5)$ & 0.7 & $(0.5,1.0)$ \\
\hline Mostly attracted to same sex & 6.9 & $(5.1,9.3)$ & 0.0 & $(0.0,0.1)$ \\
\hline Only attracted to same sex & 11.6 & $(9.6,13.9)$ & 0.2 & $(0.1,0.2)$ \\
\hline \multicolumn{5}{|l|}{ Sex $* * *$} \\
\hline Female & 76.0 & $(72.8,78.9)$ & 44.1 & $(42.8,45.5)$ \\
\hline Male & 24.0 & $(21.1,27.2)$ & 55.9 & $(54.5,57.2)$ \\
\hline \multicolumn{5}{|l|}{ Race/ethnicity** } \\
\hline White, non-Hispanic & 61.7 & $(58.0,65.3)$ & 56.6 & $(54.2,59.0)$ \\
\hline Hispanic & 17.3 & $(14.6,20.3)$ & 20.3 & $(18.3,22.5)$ \\
\hline Black, non-Hispanic & 13.2 & $(11.0,15.7)$ & 12.4 & $(11.0,13.9)$ \\
\hline Other, non-Hispanic & 7.9 & $(6.2,9.9)$ & 10.7 & $(9.4,12.2)$ \\
\hline \multicolumn{5}{|l|}{ Residence* } \\
\hline Principal MSA city & 40.7 & $(36.4,45.2)$ & 35.9 & $(32.6,39.2)$ \\
\hline Other MSA & 44.1 & $(39.7,48.5)$ & 48.9 & $(45.5,52.3)$ \\
\hline Not MSA & 15.2 & $(11.7,19.6)$ & 15.3 & $(12.3,18.8)$ \\
\hline \multicolumn{5}{|l|}{ Nativity*** } \\
\hline US-born & 92.3 & $(90.3,93.9)$ & 82.3 & $(80.8,83.6)$ \\
\hline Foreign-born & 7.7 & $(6.1,9.7)$ & 17.7 & $(16.4,19.2)$ \\
\hline \multicolumn{5}{|l|}{ Education $* * * *$} \\
\hline Some high school or less & 5.9 & $(4.9,7.3)$ & 10.2 & $(9.2,11.3)$ \\
\hline High school diploma or GED & 26.5 & $(23.5,29.7)$ & 25.8 & $(24.3,27.4)$ \\
\hline Some college & 27.0 & $(24.1,30.0)$ & 20.1 & $(18.9,21.3)$ \\
\hline College or graduate degree & 40.6 & $(37.1,44.2)$ & 43.9 & $(41.7,46.1)$ \\
\hline \multicolumn{5}{|l|}{ Insurance $* * *$} \\
\hline Private or Medi-Gap & 54.9 & $(51.2,58.5)$ & 60.5 & $(58.4,62.4)$ \\
\hline Medicaid, CHIP, State-sponsored & 19.6 & $(17.2,22.2)$ & 13.2 & $(12.1,14.4)$ \\
\hline Medicare, military, government & 5.2 & $(4.0,6.9)$ & 5.1 & $(4.2,6.2)$ \\
\hline Single-service, Indian Health Service, not covered & 20.2 & $(17.7,23.1)$ & 21.2 & $(19.7,22.8)$ \\
\hline \multicolumn{5}{|l|}{ Household income, \% of federal poverty level*** } \\
\hline $0-99$ & 22.6 & $(19.8,25.5)$ & 18.8 & $(17.6,20.0)$ \\
\hline 100-299 & 42.6 & $(39.3,45.9)$ & 39.0 & $(37.4,40.6)$ \\
\hline$>\mathbf{3 0 0} \%$ & 34.8 & $(31.4,38.4)$ & 42.2 & $(40.3,44.2)$ \\
\hline \multicolumn{5}{|l|}{ Self-rated health $* * *$} \\
\hline Poor & 1.9 & $(1.1,3.3)$ & 0.8 & $(0.6,1.1)$ \\
\hline Fair & 7.2 & $(5.9,8.7)$ & 4.9 & $(4.3,5.5)$ \\
\hline Good & 30.2 & $(27.2,33.3)$ & 23.9 & $(22.7,25.1)$ \\
\hline Very good & 36.7 & $(33.7,39.9)$ & 39.7 & $(38.4,41.0)$ \\
\hline Excellent & 24.0 & $(21.1,27.2)$ & 30.7 & $(29.4,32.1)$ \\
\hline \multicolumn{5}{|l|}{ BMI* } \\
\hline$<18.5$ (Underweight) & 1.0 & $(0.6,1.6)$ & 1.4 & $(1.1,1.8)$ \\
\hline 18.5-24.9 (Normal) & 38.1 & $(34.7,41.5)$ & 36.5 & $(35.1,37.9)$ \\
\hline 25.0-29.9 (Overweight) & 28.6 & $(25.6,31.9)$ & 33.0 & $(31.6,34.4)$ \\
\hline$>30.0$ (Obese) & 32.3 & $(29.2,35.6)$ & 29.1 & $(27.7,30.5)$ \\
\hline \multicolumn{5}{|l|}{ Access to Usual Source of Care $* * *$} \\
\hline Yes & 80.8 & $(78.0,83.3)$ & 73.7 & $(72.3,75.1)$ \\
\hline No & 19.2 & $(16.7,22.0)$ & 26.3 & $(24.9,27.7)$ \\
\hline \multicolumn{5}{|l|}{ Cycle } \\
\hline 2011-2013 & 23.6 & $(20.7,26.8)$ & 25.3 & $(22.8,28.0)$ \\
\hline 2013-2015 & 24.2 & $(21.2,27.4)$ & 26.8 & $(24.3,29.5)$ \\
\hline 2015-2017 & 25.8 & $(22.3,29.7)$ & 23.5 & $(21.1,26.2)$ \\
\hline 2017-2019 & 26.4 & $(23.2,29.9)$ & 24.4 & $(21.9,27.0)$ \\
\hline
\end{tabular}

${ }^{\mathrm{a}} \mathrm{n}=$ sample number and percent without using svy command; Mean and $95 \% \mathrm{CI}$ were conducted using svy command.

${ }^{*} \mathrm{p}<0.05,{ }^{*} \mathrm{p}<0.01,{ }^{* * *} \mathrm{p}<0.001 ; p$-values were determined using the "Pearson $\chi 2$ test with the Rao and Scott (1981, 1984 ) second-order correction" or the $F$ test (the default output from the Stata svy tabulate command)

(Stata.com n.d.:11)

Note. Sample does not include pregnant females or those missing values listwise on these variables. MSA = metropolitan statistical area; BMI = body mass index; not all strata included in each cell if missing subpopulation members. 

$(\mathbf{N}=\mathbf{1 3 , 0 3 8 )}$

\begin{tabular}{|c|c|c|c|c|c|c|c|c|}
\hline & \multirow[b]{2}{*}{$\begin{array}{l}\text { Number in } \\
\text { thousands }\end{array}$} & \multicolumn{7}{|c|}{ Self-Rated Health } \\
\hline & & $\begin{array}{l}\text { \% of Total } \\
\text { Sample }\end{array}$ & Poor & Fair & Good & Very Good & Excellent & $\begin{array}{c}\text { *Outcome sig. } \\
\text { differences }\end{array}$ \\
\hline Total Adult Sample & 13,038 & 100.0 & $1.0 \%$ & $6.3 \%$ & $25.6 \%$ & $38.4 \%$ & $28.6 \%$ & \\
\hline \multicolumn{9}{|l|}{ Sexual Identity } \\
\hline Heterosexual $^{\mathrm{A}}$ & 12,056 & 92.5 & $0.9 \%$ & $6.0 \%$ & $25.2 \%$ & $38.8 \%$ & $29.1 \%$ & $\mathrm{C}$ \\
\hline Homosexual, gay, or lesbian ${ }^{B}$ & 306 & 2.3 & $1.6 \%$ & $6.2 \%$ & $27.8 \%$ & $36.6 \%$ & $27.8 \%$ & $\mathrm{C}$ \\
\hline Bisexual $^{\mathrm{C}}$ & 676 & 5.2 & $2.4 \%$ & $11.8 \%$ & $32.0 \%$ & $32.8 \%$ & $21.0 \%$ & $\mathrm{~A}, \mathrm{~B}$ \\
\hline \multicolumn{9}{|l|}{ Sexual Behavior } \\
\hline No sexual experience with same-sex partner ${ }^{A}$ & 11,083 & 85.0 & $0.9 \%$ & $5.8 \%$ & $24.5 \%$ & $39.0 \%$ & $29.8 \%$ & $\mathrm{~B}$ \\
\hline Sexual experience with same-sex partner ${ }^{B}$ & 1,955 & 15.0 & $1.9 \%$ & $9.2 \%$ & $31.8 \%$ & $35.2 \%$ & $21.9 \%$ & A \\
\hline \multicolumn{9}{|l|}{ Sexual Attraction } \\
\hline Only attracted to opposite $\operatorname{sex}^{\mathrm{A}}$ & 10,885 & 83.5 & $0.9 \%$ & $5.9 \%$ & $24.6 \%$ & $38.6 \%$ & $29.9 \%$ & $\mathrm{~B}, \mathrm{C}$ \\
\hline Mostly attracted to opposite $\operatorname{sex}^{B}$ & 1,350 & 10.4 & $1.2 \%$ & $7.5 \%$ & $30.9 \%$ & $38.5 \%$ & $21.9 \%$ & $\mathrm{~A}, \mathrm{C}$ \\
\hline Equally attracted to males $\&$ females ${ }^{C}$ & 444 & 3.4 & $2.3 \%$ & $12.8 \%$ & $32.0 \%$ & $32.9 \%$ & $20.1 \%$ & $\mathrm{~A}, \mathrm{~B}, \mathrm{E}$ \\
\hline Mostly attracted to same sex ${ }^{D}$ & 138 & 1.1 & $2.9 \%$ & $7.3 \%$ & $31.2 \%$ & $38.4 \%$ & $20.3 \%$ & \\
\hline Only attracted to same $\operatorname{sex}^{\mathrm{E}}$ & 221 & 1.7 & $1.4 \%$ & $6.3 \%$ & $26.24 \%$ & $37.6 \%$ & $28.5 \%$ & $\mathrm{C}$ \\
\hline \multicolumn{9}{|l|}{ Sex } \\
\hline Female $^{A}$ & 7,116 & 54.6 & $1.1 \%$ & $7.5 \%$ & $26.9 \%$ & $36.9 \%$ & $27.6 \%$ & $\mathrm{~B}$ \\
\hline \multirow{3}{*}{ Male $^{\text {B }}$} & 5,922 & 45.4 & $0.9 \%$ & $4.9 \%$ & $24.2 \%$ & $40.3 \%$ & $29.8 \%$ & A \\
\hline & & \multicolumn{7}{|c|}{ BMI } \\
\hline & $\begin{array}{l}\text { Number in } \\
\text { thousands }\end{array}$ & $\begin{array}{c}\% \text { of Total } \\
\text { Sample }\end{array}$ & $\begin{array}{c}<18.5 \\
\text { (Underweight) }\end{array}$ & $\begin{array}{c}20-24 \\
\text { (Normal) }\end{array}$ & $\begin{array}{c}25.0-29.9 \\
\text { (Overweight) }\end{array}$ & $\begin{array}{c}30-50 \\
\text { (Obese) }\end{array}$ & & $\begin{array}{c}\text { *Outcome sig. } \\
\text { differences }\end{array}$ \\
\hline Total Adult Sample & 13,038 & 100.0 & $1.4 \%$ & $35.2 \%$ & $30.8 \%$ & $32.7 \%$ & & \\
\hline \multicolumn{9}{|l|}{ Sexual Identity } \\
\hline Heterosexual $^{\mathrm{A}}$ & 12,056 & 92.5 & $1.4 \%$ & $35.3 \%$ & $31.1 \%$ & $32.2 \%$ & & $\mathrm{C}$ \\
\hline Homosexual, gay, or lesbian ${ }^{B}$ & 306 & 2.3 & $1.6 \%$ & $37.6 \%$ & $28.4 \%$ & $32.4 \%$ & & $\mathrm{C}$ \\
\hline Bisexual $^{\mathrm{C}}$ & 676 & 5.2 & $1.2 \%$ & $30.8 \%$ & $26.2 \%$ & $41.9 \%$ & & $\mathrm{~A}, \mathrm{~B}$ \\
\hline \multicolumn{9}{|l|}{ Sexual Behavior } \\
\hline No sexual experience with same-sex partner ${ }^{A}$ & 11,083 & 85.0 & $1.4 \%$ & $35.2 \%$ & $31.3 \%$ & $32.1 \%$ & & $\mathrm{~B}$ \\
\hline Sexual experience with same-sex partner $^{B}$ & 1,955 & 15.0 & $1.3 \%$ & $34.8 \%$ & $27.9 \%$ & $36.0 \%$ & & A \\
\hline \multicolumn{9}{|l|}{ Sexual Attraction } \\
\hline Only attracted to opposite $\operatorname{sex}^{A}$ & 10,885 & 83.5 & $1.3 \%$ & $35.0 \%$ & $31.7 \%$ & $32.0 \%$ & & $\mathrm{~B}, \mathrm{C}$ \\
\hline Mostly attracted to opposite $\operatorname{sex}^{B}$ & 1,350 & 10.4 & $1.5 \%$ & $37.4 \%$ & $26.8 \%$ & $34.3 \%$ & & $\mathrm{~A}, \mathrm{C}$ \\
\hline Equally attracted to males $\&$ females ${ }^{C}$ & 444 & 3.4 & $1.8 \%$ & $29.5 \%$ & $23.4 \%$ & $45.3 \%$ & & $\mathrm{~A}, \mathrm{~B}, \mathrm{D}, \mathrm{E}$ \\
\hline Mostly attracted to same sex ${ }^{D}$ & 138 & 1.1 & $0.7 \%$ & $37.0 \%$ & $31.2 \%$ & $31.2 \%$ & & $\mathrm{C}$ \\
\hline Only attracted to same $\operatorname{sex}^{\mathrm{E}}$ & 221 & 1.7 & $1.8 \%$ & $38.9 \%$ & $26.2 \%$ & $33.0 \%$ & & $\mathrm{C}$ \\
\hline \multicolumn{9}{|l|}{ Sex } \\
\hline Female $^{A}$ & 7,116 & 54.6 & $1.7 \%$ & $37.4 \%$ & $25.1 \%$ & $35.9 \%$ & & $\mathrm{~B}$ \\
\hline \multirow[t]{3}{*}{ Male $^{\text {B }}$} & 5,922 & 45.4 & $1.0 \%$ & $32.5 \%$ & $37.7 \%$ & $28.9 \%$ & & A \\
\hline & \multicolumn{7}{|c|}{ Access to Usual Source of Healthcare } & \\
\hline & $\begin{array}{l}\text { Number in } \\
\text { thousands }\end{array}$ & $\begin{array}{c}\% \text { of Total } \\
\text { Sample }\end{array}$ & Yes & No & & & & $\begin{array}{c}* \text { Outcome sig. } \\
\text { differences }\end{array}$ \\
\hline Total Adult Sample & 13,038 & 100.0 & $75.0 \%$ & $25.0 \%$ & & & & \\
\hline \multicolumn{9}{|l|}{ Sexual Identity } \\
\hline Heterosexual $^{\mathrm{A}}$ & 12,056 & 92.5 & $75.7 \%$ & $25.3 \%$ & & & & $\mathrm{~B}, \mathrm{C}$ \\
\hline Homosexual, gay, or lesbian ${ }^{B}$ & 306 & 2.3 & $79.7 \%$ & $20.3 \%$ & & & & A \\
\hline Bisexual $^{\mathrm{C}}$ & 676 & 5.2 & $79.1 \%$ & $20.9 \%$ & & & & A \\
\hline \multicolumn{9}{|l|}{ Sexual Behavior } \\
\hline No sexual experience with same-sex partner ${ }^{A}$ & 11,083 & 85.0 & $74.2 \%$ & $25.8 \%$ & & & & $\mathrm{~B}$ \\
\hline Sexual experience with same-sex partner ${ }^{B}$ & 1,955 & 15.0 & $79.6 \%$ & $20.4 \%$ & & & & A \\
\hline Sexual Attraction & & & & & & & & \\
\hline Only attracted to opposite $\operatorname{sex}^{\mathrm{A}}$ & 10,885 & 83.5 & $74.3 \%$ & $25.7 \%$ & & & & $\mathrm{~B}, \mathrm{C}, \mathrm{D}$ \\
\hline Mostly attracted to opposite $\operatorname{sex}^{B}$ & 1,350 & 10.4 & $77.9 \%$ & $22.2 \%$ & & & & A, D \\
\hline Equally attracted to males $\&$ females ${ }^{C}$ & 444 & 3.4 & $80.4 \%$ & $19.6 \%$ & & & & A \\
\hline Mostly attracted to same $\operatorname{sex}^{\mathrm{D}}$ & 138 & 1.1 & $86.2 \%$ & $13.8 \%$ & & & & $\mathrm{~A}, \mathrm{~B}, \mathrm{E}$ \\
\hline Only attracted to same $\operatorname{sex}^{E}$ & 221 & 1.7 & $76.5 \%$ & $23.5 \%$ & & & & $\mathrm{D}$ \\
\hline Sex & & & & & & & & \\
\hline Female $^{\mathbf{A}}$ & 7,116 & 54.6 & $83.4 \%$ & $16.6 \%$ & & & & $\mathrm{~B}$ \\
\hline Male $^{B}$ & 5,922 & 45.4 & $65.0 \%$ & $35.0 \%$ & & & & A \\
\hline
\end{tabular}

Note. Table presents unweighted data analyses. Row totals may not equal 100.0 due to rounding.

This sample does not include pregnant females or those missing values listwise on these and other variables explored in later regression analyses.

*Items in same greyscale were grouped together in above Pearson $\chi^{2}$ tests due to small cell sizes and prior examples in the literature.

Superscripts denote a Pearson $\chi^{2}$ test of $p<0.05$ between that category and its corresponding superscript. Fisher's exact two-tailed test also run for $2 \times 2$ tables; results are consistent. 
TABLE 3-E. CH. 3 REGRESSIONS: IDENTITY: 25-34

Table 3-E. Regressions of Health Outcomes on Sexual Identity for Adults Aged 25-34 (N=13,038)

\begin{tabular}{|c|c|c|c|c|c|c|c|c|}
\hline & \multicolumn{2}{|c|}{ Self-Rated Health } & \multicolumn{2}{|c|}{ Overweight } & \multicolumn{2}{|c|}{ Obese } & \multicolumn{2}{|c|}{ Usual Source of Care } \\
\hline & OR & $95 \%$ CI & RRR & $95 \%$ CI & RRR & $95 \%$ CI & OR & $95 \%$ CI \\
\hline Intercept & $15.41 * * *$ & $(9.20,25.82)$ & $0.52 * * *$ & $(0.39,0.71)$ & $0.66^{*}$ & $(0.48,0.91)$ & $3.65 * * *$ & $(2.65,5.03)$ \\
\hline \multicolumn{9}{|l|}{ Sexual Identity } \\
\hline Heterosexual (ref) & 1.0 & & 1.0 & & 1.0 & & 1.0 & \\
\hline Homosexual, gay, or lesbian & 0.67 & $(0.34,1.35)$ & 0.79 & $(0.48,1.28)$ & 0.81 & $(0.56,1.16)$ & $1.57^{*}$ & $(1.06,2.34)$ \\
\hline Bisexual & $0.56^{* * *}$ & $(0.42,0.76)$ & 1.17 & $(0.86,1.60)$ & $1.41^{*}$ & $(1.06,1.87)$ & 0.92 & $(0.68,1.24)$ \\
\hline \multicolumn{9}{|l|}{ Survey Cycle } \\
\hline 2011-2013 (ref) & 1.0 & & 1.0 & & 1.0 & & 1.0 & \\
\hline 2013-2015 & 0.81 & $(0.61,1.07)$ & 0.90 & $(0.75,1.08)$ & 0.99 & $(0.83,1.17)$ & 0.94 & $(0.77,1.14)$ \\
\hline 2015-2017 & 0.95 & $(0.67,1.34)$ & 0.87 & $(0.73,1.04)$ & 1.09 & $(0.89,1.33)$ & 1.00 & $(0.82,1.22)$ \\
\hline 2017-2019 & 0.81 & $(0.60,1.08)$ & 1.07 & $(0.90,1.26)$ & 1.14 & $(0.93,1.40)$ & 0.91 & $(0.72,1.14)$ \\
\hline \multicolumn{9}{|l|}{ Sex } \\
\hline Female (ref) & 1.0 & & 1.0 & & 1.0 & & 1.0 & \\
\hline Male & 1.08 & $(0.86,1.34)$ & 2.20 *** & $(1.92,2.51)$ & $1.45^{* * *}$ & $(1.26,1.67)$ & $0.36 * * *$ & $(0.31,0.41)$ \\
\hline \multicolumn{9}{|l|}{ Race/ethnicity } \\
\hline White, non-Hispanic (ref) & 1.0 & & 1.0 & & 1.0 & & 1.0 & \\
\hline Hispanic & 0.84 & $(0.64,1.10)$ & $1.40 * *$ & $(1.16,1.70)$ & $1.86^{* * *}$ & $(1.55,2.23)$ & 1.02 & $(0.85,1.24)$ \\
\hline Black, non-Hispanic & 1.08 & $(0.80,1.44)$ & $1.25^{*}$ & $(1.05,1.49)$ & $1.91 * * *$ & $(1.62,2.25)$ & 1.17 & $(0.95,1.44)$ \\
\hline Other, non-Hispanic & 1.03 & $(0.71,1.48)$ & 0.82 & $(0.66,1.03)$ & 0.87 & $(0.69,1.09)$ & 1.16 & $(0.93,1.46)$ \\
\hline \multicolumn{9}{|l|}{ Nativity } \\
\hline US-born (ref) & 1.0 & & 1.0 & & 1.0 & & 1.0 & \\
\hline Foreign-born & $1.48 * *$ & $(1.11,1.99)$ & 0.84 & $(0.69,1.01)$ & $0.47 * * *$ & $(0.38,0.57)$ & 0.84 & $(0.70,1.00)$ \\
\hline \multicolumn{9}{|l|}{ Residence } \\
\hline Principal MSA city (ref) & 1.0 & & 1.0 & & 1.0 & & 1.0 & \\
\hline Other MSA & 0.87 & $(0.71,1.07)$ & 1.14 & $(1.00,1.30)$ & 1.12 & $(0.99,1.28)$ & 1.15 & $(0.99,1.34)$ \\
\hline Not MSA & 0.89 & $(0.63,1.25)$ & 1.10 & $(0.90,1.34)$ & $1.36^{* *}$ & $(1.10,1.67)$ & $1.56 * * *$ & $(1.24,1.98)$ \\
\hline \multicolumn{9}{|l|}{ Education } \\
\hline Some high school or less (ref) & 1.0 & & 1.0 & & 1.0 & & 1.0 & \\
\hline High school diploma or GED & $1.71^{* * *}$ & $(1.30,2.25)$ & 1.16 & $(0.91,1.48)$ & 1.13 & $(0.90,1.42)$ & 1.09 & $(0.87,1.37)$ \\
\hline Some college & $2.41^{* * *}$ & $(1.78,3.25)$ & 1.12 & $(0.87,1.44)$ & 1.23 & $(0.97,1.56)$ & $1.42 * *$ & $(1.10,1.83)$ \\
\hline College or graduate degree & $3.08 * * *$ & $(2.21,4.30)$ & 0.89 & $(0.71,1.13)$ & $0.78 *$ & $(0.61,1.00)$ & $1.55^{* *}$ & $(1.19,2.02)$ \\
\hline \multicolumn{9}{|l|}{ Insurance } \\
\hline Private or Medi-Gap (ref) & 1.0 & & 1.0 & & 1.0 & & 1.0 & \\
\hline Medicaid, CHIP, State-sponsored & $0.57 * * *$ & $(0.43,0.76)$ & 1.07 & $(0.85,1.35)$ & 1.12 & $(0.91,1.36)$ & 1.08 & $(0.85,1.36)$ \\
\hline Medicare, military, government & $0.51^{* *}$ & $(0.34,0.77)$ & 1.09 & $(0.84,1.41)$ & 0.82 & $(0.59,1.15)$ & $1.47 *$ & $(1.07,2.03)$ \\
\hline Single-service, Indian Health Service, not covered & $0.61 * *$ & $(0.46,0.82)$ & 0.91 & $(0.77,1.07)$ & 0.96 & $(0.79,1.16)$ & $0.29 * * *$ & $(0.25,0.35)$ \\
\hline \multicolumn{9}{|l|}{ Household income, \% of federal poverty level } \\
\hline $0-99$ (ref) & 1.0 & & 1.0 & & 1.0 & & 1.0 & \\
\hline $100-299$ & $1.52 * *$ & $(1.18,1.97)$ & 1.04 & $(0.87,1.25)$ & 0.97 & $(0.82,1.16)$ & $1.21 *$ & $(1.02,1.44)$ \\
\hline$>\mathbf{3 0 0} \%$ & $1.50^{*}$ & $(1.08,2.08)$ & 1.07 & $(0.86,1.33)$ & $0.69 * * *$ & $(0.57,0.84)$ & $1.28 *$ & $(1.04,1.58)$ \\
\hline \multicolumn{9}{|l|}{ Access to Usual Source of Care } \\
\hline Yes (ref) & 1.0 & & 1.0 & & 1.0 & & & \\
\hline No & $0.69^{* *}$ & $(0.54,0.87)$ & $0.84 *$ & $(0.72,0.97)$ & $0.75 * *$ & $(0.64,0.89)$ & & \\
\hline \multicolumn{9}{|l|}{ BMI } \\
\hline 18.5-24.9 (Normal) (ref) & 1.0 & & & & & & 1.0 & \\
\hline$<18.5$ (Underweight) & 0.56 & $(0.27,1.16)$ & & & & & 0.84 & $(0.52,1.39)$ \\
\hline 25.0-29.9 (Overweight) & 0.99 & $(0.76,1.28)$ & & & & & $1.18^{*}$ & $(1.01,1.37)$ \\
\hline$>30.0$ (Obese) & $0.48 * * *$ & $(0.38,0.61)$ & & & & & $1.30 * *$ & $(1.11,1.52)$ \\
\hline
\end{tabular}

Note. Sample does not include pregnant females or those missing values listwise on these variables.

Self-rated general health $=0$ ) Poor, Fair 1) Good, Very good, Excellent. Logistic regression

The base outcome for the overweight and obese models is body mass index (BMI) that is low/normal ( $<25)$; overweight BMI is $25-29$, and obese BMI is $>30$. Multinomial regression.

Access to a usual source of care $=0$ ) No 1) Yes. Logistic regression.

$\mathrm{OR}=$ odds ratio $\mathrm{RRR}=$ relative risk ratio $\mathrm{CI}=$ confidence interval; $\mathrm{MSA}=$ metropolitan statistical area.

The $p$-values are determined by the "Pearson $\chi^{2}$ test with the Rao and Scott $(1981,1984)$ second-order correction" or the $F$ test (the default output from the Stata svy

tabulate command); ${ }^{*} p<0.05, * * p<0.01, * * * p<0.001$ 
TABLE 3-F. CH. 3 REGRESSIONS: ATTRACTION: 25-34

Table 3-F. Regressions of Health Outcomes on Sexual Attraction for Adults Aged 25-34 (N=13,038)

\begin{tabular}{|c|c|c|c|c|c|c|c|c|}
\hline & \multicolumn{2}{|c|}{ Self-Rated Health } & \multicolumn{2}{|c|}{ Overweight } & \multicolumn{2}{|c|}{ Obese } & \multicolumn{2}{|c|}{ Usual Source of Care } \\
\hline & OR & $95 \% \mathrm{CI}$ & RRR & $95 \% \mathrm{CI}$ & RRR & $95 \% \mathrm{CI}$ & OR & $95 \% \mathrm{CI}$ \\
\hline Intercept & $15.73 * * *$ & $(9.32,26.56)$ & $0.54 * * *$ & $(0.40,0.73)$ & $0.67 *$ & $(0.49,0.92)$ & $3.80 * * *$ & $(2.77,5.23)$ \\
\hline \multicolumn{9}{|l|}{ Sexual Attraction } \\
\hline Only attracted to opposite sex (ref) & 1.0 & & 1.0 & & 1.0 & & 1.0 & \\
\hline Mostly attracted to opposite sex & 0.76 & $(0.56,1.04)$ & 0.93 & $(0.76,1.15)$ & 1.03 & $(0.83,1.27)$ & 0.82 & $(0.67,1.01)$ \\
\hline Equally attracted to males $\&$ females & $0.54 * * *$ & $(0.39,0.74)$ & 1.00 & $(0.68,1.46)$ & 1.36 & $(0.96,1.92)$ & 0.77 & $(0.53,1.13)$ \\
\hline Mostly attracted to same sex & 0.59 & $(0.29,1.20)$ & 0.91 & $(0.44,1.87)$ & 1.04 & $(0.63,1.71)$ & $3.20 * *$ & $(1.44,7.11)$ \\
\hline Only attracted to same sex & 0.68 & $(0.28,1.67)$ & 0.79 & $(0.45,1.40)$ & 0.74 & $(0.46,1.18)$ & 1.15 & $(0.74,1.81)$ \\
\hline \multicolumn{9}{|l|}{ Survey Cycle } \\
\hline 2011-2013 (ref) & 1.0 & & 1.0 & & 1.0 & & 1.0 & \\
\hline 2013-2015 & 0.81 & $(0.61,1.07)$ & 0.90 & $(0.75,1.08)$ & 0.98 & $(0.83,1.17)$ & 0.94 & $(0.77,1.15)$ \\
\hline 2015-2017 & 0.94 & $(0.66,1.34)$ & 0.88 & $(0.74,1.04)$ & 1.09 & $(0.89,1.34)$ & 1.00 & $(0.82,1.22)$ \\
\hline 2017-2019 & 0.81 & $(0.60,1.08)$ & 1.07 & $(0.91,1.27)$ & 1.15 & $(0.94,1.41)$ & 0.92 & $(0.73,1.16)$ \\
\hline \multicolumn{9}{|l|}{ Sex } \\
\hline Female (ref) & 1.0 & & 1.0 & & 1.0 & & 1.0 & \\
\hline Male & 1.05 & $(0.84,1.32)$ & $2.16^{* * *}$ & $(1.89,2.47)$ & $1.44 * * *$ & $(1.25,1.67)$ & $0.34 * * *$ & $(0.30,0.39)$ \\
\hline \multicolumn{9}{|l|}{ Race/ethnicity } \\
\hline White, non-Hispanic (ref) & 1.0 & & 1.0 & & 1.0 & & 1.0 & \\
\hline Hispanic & 0.84 & $(0.64,1.10)$ & $1.40 * *$ & $(1.15,1.69)$ & $1.85^{* * *}$ & $(1.54,2.22)$ & 1.01 & $(0.84,1.22)$ \\
\hline Black, non-Hispanic & 1.07 & $(0.80,1.43)$ & $1.24 *$ & $(1.04,1.49)$ & $1.91 * * *$ & $(1.62,2.25)$ & 1.16 & $(0.94,1.43)$ \\
\hline Other, non-Hispanic & 1.02 & $(0.71,1.47)$ & 0.82 & $(0.66,1.02)$ & 0.87 & $(0.69,1.09)$ & 1.16 & $(0.92,1.46)$ \\
\hline \multicolumn{9}{|l|}{ Nativity } \\
\hline US-born (ref) & 1.0 & & 1.0 & & 1.0 & & 1.0 & \\
\hline Foreign-born & $1.47 *$ & $(1.10,1.98)$ & 0.83 & $(0.69,1.00)$ & $0.46 * * *$ & $(0.38,0.57)$ & $0.84 *$ & $(0.70,1.00)$ \\
\hline \multicolumn{9}{|l|}{ Residence } \\
\hline Principal MSA city (ref) & 1.0 & & 1.0 & & 1.0 & & 1.0 & \\
\hline Other MSA & 0.87 & $(0.70,1.07)$ & 1.14 & $(1.00,1.30)$ & 1.12 & $(0.99,1.28)$ & 1.15 & $(0.99,1.34)$ \\
\hline Not MSA & 0.89 & $(0.63,1.25)$ & 1.10 & $(0.90,1.34)$ & $1.36^{* *}$ & $(1.10,1.67)$ & $1.55^{* * *}$ & $(1.23,1.96)$ \\
\hline \multicolumn{9}{|l|}{ Education } \\
\hline Some high school or less (ref) & 1.0 & & 1.0 & & 1.0 & & 1.0 & \\
\hline High school diploma or GED & $1.72 * * *$ & $(1.31,2.26)$ & 1.16 & $(0.91,1.48)$ & 1.13 & $(0.90,1.41)$ & 1.09 & $(0.86,1.38)$ \\
\hline Some college & $2.46^{* * *}$ & $(1.82,3.31)$ & 1.12 & $(0.87,1.44)$ & 1.22 & $(0.97,1.55)$ & $1.42 * *$ & $(1.10,1.83)$ \\
\hline College or graduate degree & $3.14 * * *$ & $(2.25,4.38)$ & 0.89 & $(0.71,1.13)$ & $0.77 *$ & $(0.60,0.99)$ & $1.55^{* *}$ & $(1.18,2.02)$ \\
\hline \multicolumn{9}{|l|}{ Insurance } \\
\hline Private or Medi-Gap (ref) & 1.0 & & 1.0 & & 1.0 & & 1.0 & \\
\hline Medicaid, CHIP, State-sponsored & $0.57 * * *$ & $(0.43,0.77)$ & 1.07 & $(0.85,1.35)$ & 1.12 & $(0.91,1.37)$ & 1.08 & $(0.85,1.36)$ \\
\hline Medicare, military, government & $0.51 * *$ & $(0.34,0.78)$ & 1.08 & $(0.83,1.41)$ & 0.82 & $(0.58,1.14)$ & $1.48^{*}$ & $(1.07,2.04)$ \\
\hline Single-service, Indian Health Service, not covered & $0.62 * *$ & $(0.46,0.82)$ & 0.91 & $(0.78,1.07)$ & 0.96 & $(0.79,1.16)$ & $0.29 * * *$ & $(0.25,0.35)$ \\
\hline \multicolumn{9}{|l|}{ Household income, \% of federal poverty level } \\
\hline $0-99$ (ref) & 1.0 & & 1.0 & & 1.0 & & 1.0 & \\
\hline $100-299$ & $1.52 * *$ & $(1.18,1.97)$ & 1.05 & $(0.88,1.25)$ & 0.97 & $(0.82,1.16)$ & $1.22 *$ & $(1.02,1.45)$ \\
\hline$>\mathbf{3 0 0} \%$ & $1.51 *$ & $(1.08,2.10)$ & 1.07 & $(0.85,1.33)$ & $0.69 * * *$ & $(0.56,0.84)$ & $1.28 *$ & $(1.04,1.59)$ \\
\hline \multicolumn{9}{|l|}{ Access to Usual Source of Care } \\
\hline Yes (ref) & 1.0 & & 1.0 & & 1.0 & & & \\
\hline No & $0.69 * *$ & $(0.54,0.87)$ & $0.84 *$ & $(0.72,0.97)$ & $0.75 * *$ & $(0.64,0.89)$ & & \\
\hline \multicolumn{9}{|l|}{ BMI } \\
\hline 18.5-24.9 (Normal) (ref) & 1.0 & & & & & & 1.0 & \\
\hline$<18.5$ (Underweight) & 0.57 & $(0.27,1.17)$ & & & & & 0.84 & $(0.51,1.38)$ \\
\hline 25.0-29.9 (Overweight) & 0.99 & $(0.76,1.28)$ & & & & & $1.18^{*}$ & $(1.01,1.37)$ \\
\hline$>30.0$ (Obese) & $0.48 * * *$ & $(0.38,0.60)$ & & & & & $1.30 * *$ & $(1.10,1.52)$ \\
\hline
\end{tabular}

Note. Sample does not include pregnant females or those missing values listwise on these variables.

Self-rated general health $=0$ ) Poor, Fair 1) Good, Very good, Excellent. Logistic regression

The base outcome for the overweight and obese models is body mass index (BMI) that is low/normal $(<25)$; overweight BMI is $25-29$, and obese BMI is $>30$. Multinomial regression.

Access to a usual source of care $=0$ ) No 1) Yes. Logistic regression.

$\mathrm{OR}=$ odds ratio $\mathrm{RRR}=$ relative risk ratio $\mathrm{CI}=$ confidence interval; $\mathrm{MSA}=$ metropolitan statistical area.

The $p$-values are determined by the "Pearson $\chi^{2}$ test with the Rao and Scott $(1981,1984)$ second-order correction" or the $F$ test (the default output from the Stata svy

tabulate command); ${ }^{*} p<0.05, * * p<0.01,{ }^{* * *} p<0.001$ 
TABLE 3-G. CH. 3 REGRESSIONS: BEHAVIOR: 25-34

Table 3-G. Regressions of Health Outcomes on Sexual Behavior for Adults Aged 25-34 (N=13,038)

\begin{tabular}{|c|c|c|c|c|c|c|c|c|}
\hline & \multicolumn{2}{|c|}{ Self-Rated Health } & \multicolumn{2}{|c|}{ Overweight } & \multicolumn{2}{|c|}{ Obese } & \multicolumn{2}{|c|}{ Usual Source of Care } \\
\hline & OR & $95 \% \mathrm{CI}$ & RRR & $95 \%$ CI & RRR & $95 \% \mathrm{CI}$ & OR & $95 \% \mathrm{CI}$ \\
\hline Intercept & $16.11 * * *$ & $(9.55,27.18)$ & $0.52 * * *$ & $(0.39,0.71)$ & $0.67 *$ & $(0.49,0.92)$ & $3.59 * * *$ & $(2.60,4.94)$ \\
\hline \multicolumn{9}{|l|}{ Sexual Behavior } \\
\hline No sexual experience with same-sex partner (ref) & 1.0 & & 1.0 & & 1.0 & & 1.0 & \\
\hline Sexual experience with same-sex partner & $0.65 * *$ & $(0.50,0.84)$ & 1.04 & $(0.84,1.28)$ & 1.05 & $(0.87,1.26)$ & 1.08 & $(0.90,1.30)$ \\
\hline \multicolumn{9}{|l|}{ Survey Cycle } \\
\hline 2011-2013 (ref) & 1.0 & & 1.0 & & 1.0 & & 1.0 & \\
\hline 2013-2015 & 0.80 & $(0.61,1.06)$ & 0.90 & $(0.74,1.08)$ & 0.98 & $(0.83,1.17)$ & 0.94 & $(0.77,1.14)$ \\
\hline 2015-2017 & 0.93 & $(0.66,1.33)$ & 0.87 & $(0.73,1.04)$ & 1.09 & $(0.89,1.33)$ & 1.00 & $(0.82,1.22)$ \\
\hline 2017-2019 & 0.79 & $(0.59,1.05)$ & 1.07 & $(0.91,1.26)$ & 1.15 & $(0.94,1.41)$ & 0.91 & $(0.72,1.14)$ \\
\hline \multicolumn{9}{|l|}{ Sex } \\
\hline Female (ref) & 1.0 & & 1.0 & & 1.0 & & 1.0 & \\
\hline Male & 1.04 & $(0.83,1.30)$ & $2.19 * * *$ & $(1.92,2.51)$ & $1.43 * * *$ & $(1.24,1.65)$ & $0.36 * * *$ & $(0.31,0.41)$ \\
\hline \multicolumn{9}{|l|}{ Race/ethnicity } \\
\hline White, non-Hispanic (ref) & 1.0 & & 1.0 & & 1.0 & & 1.0 & \\
\hline Hispanic & 0.85 & $(0.65,1.10)$ & $1.40 * *$ & $(1.15,1.69)$ & $1.84 * * *$ & $(1.53,2.21)$ & 1.03 & $(0.86,1.25)$ \\
\hline Black, non-Hispanic & 1.07 & $(0.80,1.43)$ & $1.25 *$ & $(1.05,1.49)$ & $1.90 * * *$ & $(1.61,2.24)$ & 1.18 & $(0.95,1.45)$ \\
\hline Other, non-Hispanic & 1.01 & $(0.71,1.45)$ & 0.82 & $(0.66,1.02)$ & 0.87 & $(0.69,1.09)$ & 1.17 & $(0.94,1.47)$ \\
\hline \multicolumn{9}{|l|}{ Nativity } \\
\hline US-born (ref) & 1.0 & & 1.0 & & 1.0 & & 1.0 & \\
\hline Foreign-born & $1.45 *$ & $(1.08,1.95)$ & 0.83 & $(0.70,1.01)$ & $0.47 * * *$ & $(0.38,0.57)$ & 0.84 & $(0.70,1.00)$ \\
\hline \multicolumn{9}{|l|}{ Residence } \\
\hline Principal MSA city (ref) & 1.0 & & 1.0 & & 1.0 & & 1.0 & \\
\hline Other MSA & 0.86 & $(0.70,1.06)$ & $1.14^{*}$ & $(1.00,1.30)$ & 1.12 & $(0.99,1.28)$ & 1.15 & $(0.99,1.34)$ \\
\hline Not MSA & 0.89 & $(0.63,1.26)$ & 1.10 & $(0.90,1.35)$ & $1.36 * *$ & $(1.10,1.67)$ & $1.57 * * *$ & $(1.24,1.98)$ \\
\hline \multicolumn{9}{|l|}{ Education } \\
\hline Some high school or less (ref) & 1.0 & & 1.0 & & 1.0 & & 1.0 & \\
\hline High school diploma or GED & $1.74 * * *$ & $(1.32,2.28)$ & 1.16 & $(0.91,1.48)$ & 1.12 & $(0.89,1.41)$ & 1.09 & $(0.87,1.38)$ \\
\hline Some college & $2.47 * * *$ & $(1.83,3.34)$ & 1.11 & $(0.86,1.43)$ & 1.23 & $(0.97,1.55)$ & $1.42 * *$ & $(1.10,1.83)$ \\
\hline College or graduate degree & $3.11 * * *$ & $(2.23,4.34)$ & 0.89 & $(0.70,1.13)$ & $0.78 *$ & $(0.61,1.00)$ & $1.55 * *$ & $(1.19,2.02)$ \\
\hline \multicolumn{9}{|l|}{ Insurance } \\
\hline Private or Medi-Gap (ref) & 1.0 & & 1.0 & & 1.0 & & 1.0 & \\
\hline Medicaid, CHIP, State-sponsored & $0.57 * * *$ & $(0.43,0.77)$ & 1.07 & $(0.85,1.35)$ & 1.12 & $(0.92,1.37)$ & 1.07 & $(0.85,1.35)$ \\
\hline Medicare, military, government & $0.51 * *$ & $(0.34,0.78)$ & 1.08 & $(0.84,1.41)$ & 0.82 & $(0.58,1.14)$ & $1.47^{*}$ & $(1.07,2.03)$ \\
\hline Single-service, Indian Health Service, not covered & $0.62 * *$ & $(0.46,0.82)$ & 0.91 & $(0.77,1.06)$ & 0.96 & $(0.79,1.16)$ & $0.29 * * *$ & $(0.25,0.35)$ \\
\hline \multicolumn{9}{|l|}{ Household income, \% of federal poverty level } \\
\hline $0-99$ (ref) & 1.0 & & 1.0 & & 1.0 & & 1.0 & \\
\hline $100-299$ & $1.52 * *$ & $(1.18,1.97)$ & 1.05 & $(0.88,1.25)$ & 0.98 & $(0.82,1.16)$ & $1.21 *$ & $(1.02,1.44)$ \\
\hline$>\mathbf{3 0 0} \%$ & $1.50 *$ & $(1.08,2.09)$ & 1.07 & $(0.86,1.33)$ & $0.69 * * *$ & $(0.57,0.84)$ & $1.28 *$ & $(1.04,1.58)$ \\
\hline \multicolumn{9}{|l|}{ Access to Usual Source of Care } \\
\hline Yes (ref) & 1.0 & & 1.0 & & 1.0 & & & \\
\hline No & $0.69 * *$ & $(0.54,0.87)$ & $0.84 *$ & $(0.72,0.98)$ & $0.76 * *$ & $(0.64,0.89)$ & & \\
\hline \multicolumn{9}{|l|}{ BMI } \\
\hline 18.5-24.9 (Normal) (ref) & 1.0 & & & & & & 1.0 & \\
\hline$<18.5$ (Underweight) & 0.56 & $(0.27,1.16)$ & & & & & 0.85 & $(0.52,1.39)$ \\
\hline 25.0-29.9 (Overweight) & 0.99 & $(0.76,1.29)$ & & & & & $1.17 *$ & $(1.10,1.37)$ \\
\hline$>30.0$ (Obese) & $0.48 * * *$ & $(0.38,0.60)$ & & & & & $1.29 * *$ & $(1.10,1.52)$ \\
\hline
\end{tabular}

Note. Sample does not include pregnant females or those missing values listwise on these variables.

Self-rated general health $=0$ ) Poor, Fair 1) Good, Very good, Excellent. Logistic regression

The base outcome for the overweight and obese models is body mass index (BMI) that is low/normal (<25); overweight BMI is $25-29$, and obese BMI is $>30$. Multinomial regression.

Access to a usual source of care $=0$ ) No 1) Yes. Logistic regression.

$\mathrm{OR}=$ odds ratio $; \mathrm{RRR}=$ relative risk ratio $; \mathrm{CI}=$ confidence interval; $\mathrm{MSA}=$ metropolitan statistical area.

The $p$-values are determined by the "Pearson $\chi^{2}$ test with the Rao and Scott $(1981,1984)$ second-order correction" or the $F$ test (the default output from the Stata svy

tabulate command); $* p<0.05, * * p<0.01, * * * p<0.001$ 
Table 3-H. Characteristics of US Adults Aged 35-44, by Sexual Identity 2011-2019 (N=10,529)

\begin{tabular}{|c|c|c|c|c|c|c|}
\hline & \multicolumn{6}{|c|}{ Sexual Identity } \\
\hline & \multicolumn{2}{|c|}{$\begin{array}{l}\text { Heterosexual or straight } \\
\qquad(\mathrm{n}=9,942 ; 94.4 \%)^{\mathrm{a}}\end{array}$} & \multicolumn{2}{|c|}{$\begin{array}{c}\text { Homosexual, gay, or } \\
\text { lesbian } \\
(\mathrm{n}=\mathbf{2 4 6} ; 2.3 \%)^{\mathrm{a}}\end{array}$} & \multicolumn{2}{|c|}{$\begin{array}{c}\text { Bisexual } \\
(\mathrm{n}=341 ; 3.2 \%)^{\mathrm{a}}\end{array}$} \\
\hline & Mean \% & $95 \% \mathrm{CI}$ & Mean \% & $95 \%$ CI & Mean \% & $95 \% \mathrm{CI}$ \\
\hline \multicolumn{7}{|l|}{ Sexual Attraction*** } \\
\hline Only attracted to opposite sex & 94.1 & $(93.4,94.8)$ & 5.4 & $(2.2,12.7)$ & 5.6 & $(3.3,9.2)$ \\
\hline Mostly attracted to opposite sex & 5.6 & $(5.0,6.4)$ & 0.6 & $(0.1,2.3)$ & 43.1 & $(34.7,51.9)$ \\
\hline Equally attracted to males \& females & 0.2 & $(0.1,0.3)$ & 2.1 & $(0.9,5.2)$ & 43.8 & $(36.2,51.6)$ \\
\hline Mostly attracted to same sex & 0.0 & $(0.0,0.0)$ & 27.0 & $(19.5,36.2)$ & 7.5 & $(4.2,13.3)$ \\
\hline Only attracted to same sex & 0.0 & $(0.0,0.1)$ & 64.8 & $(55.9,72.8)$ & 0.0 & $(\mathrm{~N} / \mathrm{A})$ \\
\hline \multicolumn{7}{|l|}{ Sexual Behavior $* * * *$} \\
\hline Sexual experience with same-sex partner & 7.1 & $(6.3,7.9)$ & 95.7 & $(91.5,97.9)$ & 87.1 & $(81.8,91.1)$ \\
\hline No sexual experience with same-sex partner & 92.9 & $(92.1,93.7)$ & 4.3 & $(2.1,8.5)$ & 12.9 & $(8.9,18.2)$ \\
\hline \multicolumn{7}{|l|}{ Sex $* * *$} \\
\hline Female & 49.5 & $(48.2,50.9)$ & 40.8 & $(32.4,49.7)$ & 77.0 & $(70.6,82.4)$ \\
\hline Male & 50.5 & $(49.1,51.8)$ & 59.2 & $(50.3,67.6)$ & 23.0 & $(17.6,29.4)$ \\
\hline \multicolumn{7}{|l|}{ Race/ethnicity } \\
\hline White, non-Hispanic & 58.6 & $(56.2,60.9)$ & 68.4 & $(59.6,76.0)$ & 63.0 & $(55.1,70.3)$ \\
\hline Hispanic & 19.7 & $(17.5,22.0)$ & 14.0 & $(9.1,20.9)$ & 15.2 & $(10.1,22.2)$ \\
\hline Black, non-Hispanic & 12.0 & $(10.7,13.4)$ & 8.3 & $(5.6,12.1)$ & 11.5 & $(7.9,16.5)$ \\
\hline Other, non-Hispanic & 9.8 & $(8.7,11.1)$ & 9.3 & $(5.2,16.0)$ & 10.3 & $(5.9,17.3)$ \\
\hline \multicolumn{7}{|l|}{ Residence $^{* *}$} \\
\hline Principal MSA city & 31.0 & $(28.3,33.9)$ & 44.9 & $(34.4,55.8)$ & 40.8 & $(32.7,49.3)$ \\
\hline Other MSA & 52.7 & $(49.4,56.0)$ & 38.7 & $(28.2,50.3)$ & 47.8 & $(39.6,56.1)$ \\
\hline Not MSA & 16.3 & $(13.2,20.0)$ & 16.5 & $(9.9,26.1)$ & 11.5 & $(7.4,17.3)$ \\
\hline \multicolumn{7}{|l|}{ Nativity*** } \\
\hline US-born & 80.0 & $(78.2,81.6)$ & 85.8 & $(78.8,90.7)$ & 92.3 & $(88.5,95.0)$ \\
\hline Foreign-born & 20.0 & $(18.4,21.8)$ & 14.2 & $(9.3,21.2)$ & 7.7 & $(5.0,11.5)$ \\
\hline \multicolumn{7}{|l|}{ Education } \\
\hline Some high school or less & 10.7 & $(9.5,12.0)$ & 8.5 & $(4.4,15.6)$ & 9.5 & $(6.2,14.3)$ \\
\hline High school diploma or GED & 24.8 & $(23.4,26.2)$ & 18.9 & $(12.3,27.9)$ & 24.7 & $(18.8,31.7)$ \\
\hline Some college & 18.2 & $(17.0,19.5)$ & 14.2 & $(8.9,21.9)$ & 22.5 & $(17.2,28.9)$ \\
\hline College or graduate degree & 46.3 & $(44.2,48.5)$ & 58.4 & $(49.3,66.9)$ & 43.3 & $(36.5,50.3)$ \\
\hline \multicolumn{7}{|l|}{ Insurance $* * *$} \\
\hline Private or Medi-Gap & 69.8 & $(68.0,71.5)$ & 69.8 & $(61.4,77.0)$ & 53.0 & $(45.5,60.3)$ \\
\hline Medicaid, CHIP, State-sponsored & 9.9 & $(9.0,11.0)$ & 14.7 & $(9.0,23.0)$ & 19.1 & $(14.6,24.7)$ \\
\hline Medicare, military, government & 4.2 & $(3.4,5.0)$ & 3.6 & $(1.4,8.6)$ & 5.0 & $(2.2,10.6)$ \\
\hline Single-service, Indian Health Service, not covered & 16.1 & $(14.8,17.6)$ & 12.0 & $(7.6,18.4)$ & 23.0 & $(17.5,29.6)$ \\
\hline \multicolumn{7}{|l|}{ Household income, \% of federal poverty level* } \\
\hline $0-99$ & 16.4 & $(15.2,17.7)$ & 17.1 & $(11.6,24.4)$ & 23.2 & $(18.2,29.1)$ \\
\hline 100-299 & 33.7 & $(32.0,35.4)$ & 25.8 & $(19.0,34.0)$ & 35.7 & $(29.3,42.7)$ \\
\hline$>300 \%$ & 49.9 & $(47.7,52.1)$ & 57.1 & $(48.4,65.4)$ & 41.1 & $(33.2,49.4)$ \\
\hline \multicolumn{7}{|l|}{ Self-rated health** } \\
\hline Poor & 1.3 & $(1.0,1.6)$ & 2.7 & $(1.2,5.7)$ & 2.4 & $(1.3,4.4)$ \\
\hline Fair & 8.3 & $(7.5,9.1)$ & 8.7 & $(5.8,13.0)$ & 12.7 & $(8.5,18.5)$ \\
\hline Good & 26.7 & $(25.3,28.1)$ & 29.2 & $(21.9,37.8)$ & 37.1 & $(30.4,44.4)$ \\
\hline Very good & 39.3 & $(37.8,40.9)$ & 33.8 & $(25.6,42.9)$ & 26.4 & $(19.4,34.8)$ \\
\hline Excellent & 24.4 & $(23.2,25.7)$ & 25.6 & $(18.4,34.6)$ & 21.5 & $(16.4,27.5)$ \\
\hline \multicolumn{7}{|l|}{ BMI } \\
\hline$<18.5$ (Underweight) & 0.8 & $(0.6,1.1)$ & 0.3 & $(0.0,1.8)$ & 0.5 & $(0.1,1.9)$ \\
\hline 18.5-24.9 (Normal) & 28.5 & $(27.1,30.0)$ & 34.8 & $(26.8,43.8)$ & 31.4 & $(24.2,39.7)$ \\
\hline 25.0-29.9 (Overweight) & 35.0 & $(33.6,36.4)$ & 28.6 & $(21.3,37.2)$ & 29.5 & $(22.6,37.4)$ \\
\hline$>30.0$ (Obese) & 35.7 & $(34.1,37.3)$ & 36.4 & $(28.1,45.6)$ & 38.6 & $(31.3,46.5)$ \\
\hline Access to Usual Source of Care & & & & & & \\
\hline Yes & 83.3 & $(82.0,84.6)$ & 86.7 & $(76.6,92.9)$ & 86.2 & $(80.7,90.3)$ \\
\hline No & 16.7 & $(15.4,18.0)$ & 13.3 & $(7.1,23.4)$ & 13.8 & $(9.7,19.3)$ \\
\hline Cycle & & & & & & \\
\hline 2011-2013 & 25.1 & $(23.0,27.3)$ & 20.4 & $(13.7,29.1)$ & 25.9 & $(19.4,33.8)$ \\
\hline 2013-2015 & 26.5 & $(24.2,29.0)$ & 20.6 & $(14.7,28.1)$ & 26.9 & $(20.9,33.9)$ \\
\hline 2015-2017 & 25.1 & $(22.9,27.3)$ & 33.4 & $(22.2,46.9)$ & 20.7 & $(15.5,26.9)$ \\
\hline 2017-2019 & 23.4 & $(21.1,25.8)$ & 25.6 & $(17.8,35.3)$ & 26.5 & $(19.9,34.4)$ \\
\hline
\end{tabular}

${ }^{a} \mathrm{n}=$ sample number and percent without using svy command; Mean and $95 \%$ CI were conducted using svy command.

${ }^{*} \mathrm{p}<0.05,{ }^{* *} \mathrm{p}<0.01,{ }^{* * *} \mathrm{p}<0.001 ; p$-values were determined using the "Pearson $\chi 2$ test with the Rao and Scott $(1981,1984)$ second-order correction" or the $F$ test (the default output from the Stata svy tabulate command) (Stata.com n.d.:11).

Note. Sample does not include pregnant females or those missing values listwise on these variables. MSA = metropolitan statistical area; $\mathrm{BMI}=$ body mass index; N/A = not applicable. not all strata included in each cell if missing subpopulation members. 
TABLE 3-I. CH. 3 SUMMARY STATISTICS: ATTRACTION: 35-44

Table 3-I. Characteristics of US Adults Aged 35-44, by Sexual Attraction 2011-2019 (N=10,529)

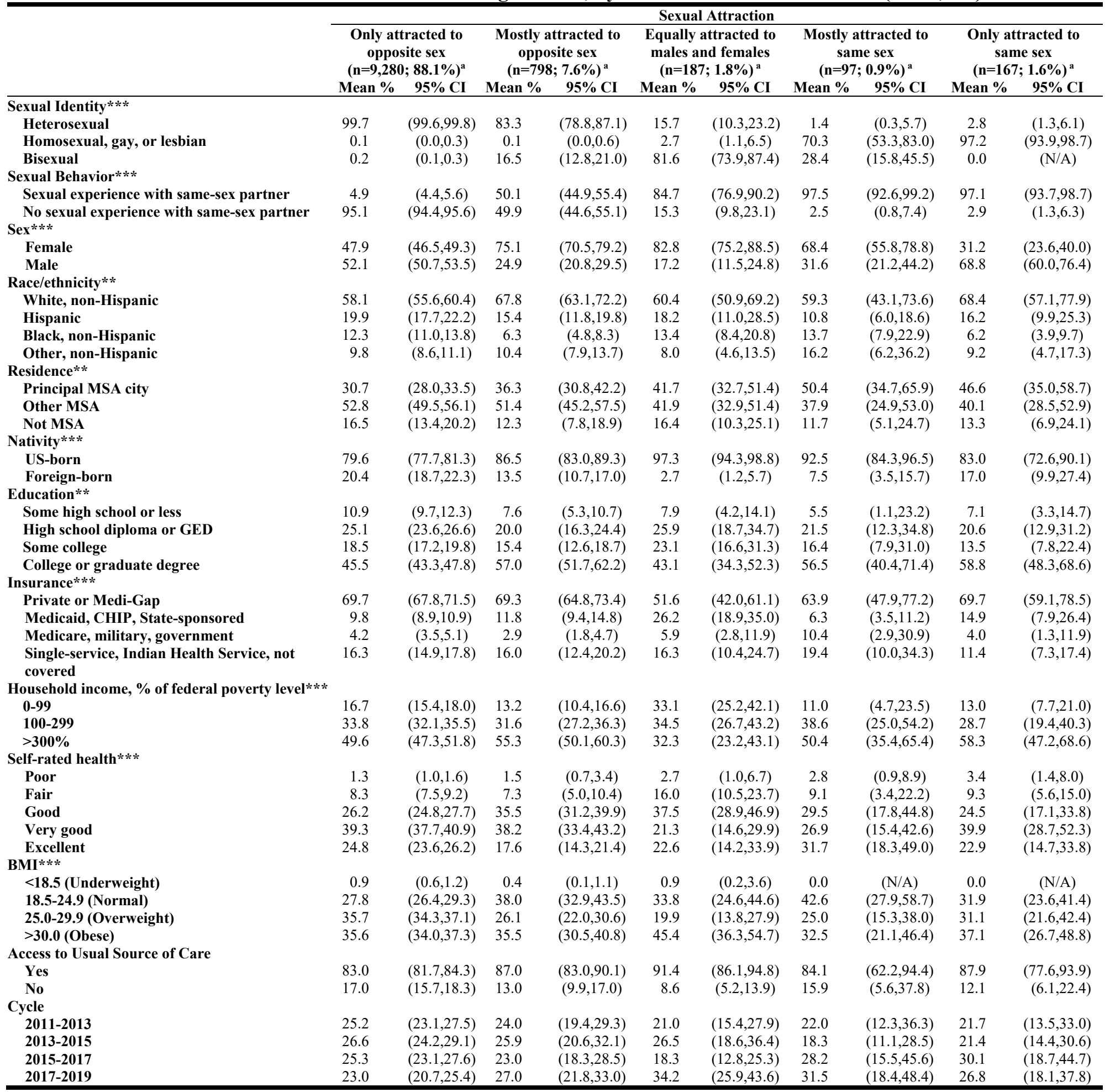

${ }^{a} \mathrm{n}=$ sample number and percent without using svy command; Mean and $95 \%$ CI were conducted using svy command.

${ }^{*} \mathrm{p}<0.05,{ }^{*} \mathrm{p}<0.01,{ }^{* * *} \mathrm{p}<0.001 ; p$-values were determined using the "Pearson $\chi 2$ test with the Rao and Scott $(1981,1984)$ second-order correction" or the $F$ test (the default output from the Stata svy tabulate command) (Stata.com n.d.:11).

Note. Sample does not include pregnant females or those missing values listwise on these variables. $\mathrm{MSA}=$ metropolitan statistical area; $\mathrm{BMI}=$ body mass index; $\mathrm{N} / \mathrm{A}=$ not applicable. not all strata included in each cell if missing subpopulation members. 
Table 3-J. Characteristics of US Adults Aged 35-44, by Sexual Behavior 2011-2019 (N=10,529)

\begin{tabular}{|c|c|c|c|c|}
\hline & \multicolumn{4}{|c|}{ Sexual Behavior } \\
\hline & \multicolumn{2}{|c|}{$\begin{array}{c}\text { Sexual experience } \\
\text { with same sex } \\
\text { partner } \\
(\mathrm{n}=1,327 ; \mathbf{1 2 . 6 \%})^{\mathrm{a}}\end{array}$} & \multicolumn{2}{|c|}{$\begin{array}{c}\text { No sexual experience } \\
\text { with same sex } \\
\text { partner } \\
(\mathrm{n}=\mathbf{9 , 2 0 2} ; \mathbf{8 7 . 4 \%} \%)^{\mathrm{a}}\end{array}$} \\
\hline & Mean \% & $95 \%$ CI & Mean \% & $95 \%$ CI \\
\hline \multicolumn{5}{|l|}{ Sexual Identity*** } \\
\hline Heterosexual & 64.2 & $(60.3,67.8)$ & 99.6 & $(99.4,99.7)$ \\
\hline Homosexual, gay, or lesbian & 15.5 & $(12.6,18.8)$ & 0.1 & $(0.0,0.2)$ \\
\hline Bisexual & 20.4 & $(17.5,23.6)$ & 0.4 & $(0.2,0.5)$ \\
\hline \multicolumn{5}{|l|}{ Sexual Attraction $* * *$} \\
\hline Only attracted to opposite sex & 42.2 & $(38.7,45.9)$ & 96.1 & $(95.5,96.7)$ \\
\hline Mostly attracted to opposite sex & 30.6 & $(27.3,34.1)$ & 3.6 & $(3.1,4.2)$ \\
\hline Equally attracted to males $\&$ females & 10.6 & $(8.6,13.0)$ & 0.2 & $(0.1,0.4)$ \\
\hline Mostly attracted to same sex & 6.1 & $(4.5,8.2)$ & 0.0 & $(0.0,0.1)$ \\
\hline Only attracted to same sex & 10.5 & $(8.3,13.2)$ & 0.0 & $(0.0,0.1)$ \\
\hline \multicolumn{5}{|l|}{ Sex $* * *$} \\
\hline Female & 72.8 & $(69.1,76.3)$ & 47.4 & $(46.0,48.8)$ \\
\hline Male & 27.2 & $(23.7,30.9)$ & 52.6 & $(51.2,54.0)$ \\
\hline \multicolumn{5}{|l|}{ Race/ethnicity $* * *$} \\
\hline White, non-Hispanic & 66.7 & $(62.8,70.3)$ & 57.9 & $(55.5,60.3)$ \\
\hline Hispanic & 12.7 & $(10.3,15.6)$ & 20.2 & $(18.0,22.7)$ \\
\hline Black, non-Hispanic & 11.0 & $(8.9,13.6)$ & 12.0 & $(10.7,13.4)$ \\
\hline Other, non-Hispanic & 9.6 & $(7.4,12.3)$ & 9.8 & $(8.6,11.2)$ \\
\hline \multicolumn{5}{|l|}{ Residence } \\
\hline Principal MSA city & 36.4 & $(32.0,41.0)$ & 30.9 & $(28.2,33.8)$ \\
\hline Other MSA & 48.5 & $(43.7,53.4)$ & 52.8 & $(49.4,56.1)$ \\
\hline Not MSA & 15.1 & $(10.7,21.1)$ & 16.3 & $(13.2,20.0)$ \\
\hline \multicolumn{5}{|l|}{ Nativity*** } \\
\hline US-born & 92.3 & $(90.0,94.2)$ & 79.0 & $(77.1,80.7)$ \\
\hline Foreign-born & 7.7 & $(5.8,10.0)$ & 21.0 & $(19.3,22.9)$ \\
\hline \multicolumn{5}{|l|}{ Education** } \\
\hline Some high school or less & 7.5 & $(5.8,9.6)$ & 11.0 & $(9.7,12.3)$ \\
\hline High school diploma or GED & 21.9 & $(18.6,25.6)$ & 25.0 & $(23.6,26.5)$ \\
\hline Some college & 20.0 & $(17.2,23.2)$ & 18.0 & $(16.8,19.4)$ \\
\hline College or graduate degree & 50.6 & $(46.1,55.1)$ & 46.0 & $(43.8,48.2)$ \\
\hline \multicolumn{5}{|l|}{ Insurance $* * *$} \\
\hline Private or Medi-Gap & 62.6 & $(58.5,66.6)$ & 70.2 & $(68.3,72.0)$ \\
\hline Medicaid, CHIP, State-sponsored & 16.0 & $(13.7,18.7)$ & 9.5 & $(8.6,10.6)$ \\
\hline Medicare, military, government & 5.0 & $(13.7,18.7)$ & 4.1 & $(3.3,5.0)$ \\
\hline Single-service, Indian Health Service, not covered & 16.4 & $(13.2,20.1)$ & 16.2 & $(14.8,17.7)$ \\
\hline \multicolumn{5}{|l|}{ Household income, \% of federal poverty level* } \\
\hline $0-99$ & 20.4 & $(17.4,23.8)$ & 16.1 & $(14.9,17.5)$ \\
\hline 100-299 & 33.4 & $(29.7,37.2)$ & 33.6 & $(31.9,35.4)$ \\
\hline$>\mathbf{3 0 0} \%$ & 46.2 & $(42.0,50.5)$ & 50.2 & $(47.9,52.5)$ \\
\hline \multicolumn{5}{|l|}{ Self-rated health $* * *$} \\
\hline Poor & 2.4 & $(1.5,3.8)$ & 1.2 & $(0.9,1.6)$ \\
\hline Fair & 11.5 & $(9.2,14.2)$ & 8.0 & $(7.2,8.9)$ \\
\hline Good & 33.2 & $(29.6,37.0)$ & 26.2 & $(24.8,27.7)$ \\
\hline Very good & 33.1 & $(29.3,37.2)$ & 39.6 & $(38.0,41.2)$ \\
\hline Excellent & 19.8 & $(16.9,23.1)$ & 24.9 & $(23.6,26.2)$ \\
\hline \multicolumn{5}{|l|}{ BMI** } \\
\hline$<18.5$ (Underweight) & 0.6 & $(0.3,1.4)$ & 0.8 & $(0.6,1.1)$ \\
\hline 18.5-24.9 (Normal) & 32.5 & $(28.8,36.3)$ & 28.2 & $(26.8,29.7)$ \\
\hline 25.0-29.9 (Overweight) & 28.0 & $(24.7,31.5)$ & 35.5 & $(34.1,37.0)$ \\
\hline$>30.0$ (Obese) & 39.0 & $(35.0,43.2)$ & 35.4 & $(33.8,37.0)$ \\
\hline \multicolumn{5}{|l|}{ Access to Usual Source of Care $* * *$} \\
\hline Yes & 88.8 & $(86.1,91.0)$ & 82.8 & $(81.5,84.1)$ \\
\hline \multirow{2}{*}{\multicolumn{5}{|c|}{ Cycle* }} \\
\hline & & & & \\
\hline 2011-2013 & 22.0 & $(18.3,26.2)$ & 25.4 & $(23.2,27.6)$ \\
\hline 2013-2015 & 25.8 & $(21.8,30.4)$ & 26.5 & $(24.1,29.0)$ \\
\hline 2015-2017 & 23.5 & $(19.6,27.8)$ & 25.3 & $(23.1,27.7)$ \\
\hline 2017-2019 & 28.7 & $(24.2,33.8)$ & 22.9 & $(20.6,25.3)$ \\
\hline
\end{tabular}

${ }^{\mathrm{a}} \mathrm{n}=$ sample number and percent without using svy command; Mean and $95 \% \mathrm{CI}$ were conducted using svy command.

${ }^{*} \mathrm{p}<0.05,{ }^{*} \mathrm{p}<0.01,{ }^{* * *} \mathrm{p}<0.001 ; p$-values were determined using the "Pearson $\chi 2$ test with the Rao and Scott (1981, 1984 ) second-order correction" or the $F$ test (the default output from the Stata svy tabulate command)

(Stata.com n.d.:11).

Note. Sample does not include pregnant females or those missing values listwise on these variables. MSA = metropolitan statistical area; BMI = body mass index; not all strata included in each cell if missing subpopulation members 
Table 3-K. Sample Characteristics of US Adults Aged 35-44 by Sexual Identity Components and Health Outcomes 2011-2019 $(\mathbf{N}=\mathbf{1 0 , 5 2 9})$

\begin{tabular}{|c|c|c|c|c|c|c|c|c|}
\hline & \multirow[b]{2}{*}{$\begin{array}{l}\text { Number in } \\
\text { thousands }\end{array}$} & \multicolumn{7}{|c|}{ Self-Rated Health } \\
\hline & & $\begin{array}{c}\text { \% of Total } \\
\text { Sample }\end{array}$ & Poor & Fair & Good & Very Good & Excellent & $\begin{array}{l}\text { *Outcome sig. } \\
\text { differences }\end{array}$ \\
\hline Total Adult Sample & 10,529 & 100.0 & $1.9 \%$ & $9.3 \%$ & $27.8 \%$ & $37.7 \%$ & $23.4 \%$ & \\
\hline \multicolumn{9}{|l|}{ Sexual Identity } \\
\hline Heterosexual $^{\mathrm{A}}$ & 9,942 & 94.4 & $1.8 \%$ & $9.1 \%$ & $27.4 \%$ & $38.2 \%$ & $23.5 \%$ & $\mathrm{~B}, \mathrm{C}$ \\
\hline Homosexual, gay, or lesbian ${ }^{B}$ & 246 & 2.3 & $4.1 \%$ & $12.2 \%$ & $28.5 \%$ & $32.1 \%$ & $23.2 \%$ & A \\
\hline Bisexual $^{\mathrm{C}}$ & 341 & 3.2 & $3.8 \%$ & $12.9 \%$ & $36.7 \%$ & $26.4 \%$ & $20.2 \%$ & A \\
\hline \multicolumn{9}{|l|}{ Sexual Behavior } \\
\hline No sexual experience with same-sex partner ${ }^{A}$ & 9,202 & 87.4 & $1.7 \%$ & $8.8 \%$ & $27.2 \%$ & $38.3 \%$ & $24.1 \%$ & $\mathrm{~B}$ \\
\hline Sexual experience with same-sex partner $^{B}$ & 1,327 & 12.6 & $3.4 \%$ & $12.4 \%$ & $31.8 \%$ & $33.8 \%$ & $18.5 \%$ & A \\
\hline \multicolumn{9}{|l|}{ Sexual Attraction } \\
\hline Only attracted to opposite $\operatorname{sex}^{\mathrm{A}}$ & 9,280 & 88.1 & $1.8 \%$ & $9.1 \%$ & $27.0 \%$ & $38.1 \%$ & $24.0 \%$ & $\mathrm{C}, \mathrm{E}$ \\
\hline Mostly attracted to opposite $\operatorname{sex}^{B}$ & 798 & 7.6 & $1.8 \%$ & $9.5 \%$ & $33.3 \%$ & $38.0 \%$ & $17.4 \%$ & $\mathrm{C}$ \\
\hline Equally attracted to males $\&$ females ${ }^{C}$ & 187 & 1.8 & $3.7 \%$ & $15.5 \%$ & $40.1 \%$ & $23.0 \%$ & $17.7 \%$ & $\mathrm{~A}, \mathrm{~B}$ \\
\hline Mostly attracted to same $\operatorname{sex}^{\mathrm{D}}$ & 97 & 0.9 & $4.1 \%$ & $8.3 \%$ & $27.8 \%$ & $29.9 \%$ & $29.9 \%$ & \\
\hline Only attracted to same $\operatorname{sex}^{\mathrm{E}}$ & 167 & 1.6 & $4.2 \%$ & $12.0 \%$ & $27.5 \%$ & $35.3 \%$ & $21.0 \%$ & A \\
\hline \multicolumn{9}{|l|}{ Sex } \\
\hline Female $^{\mathrm{A}}$ & 5,779 & 54.9 & $2.3 \%$ & $9.9 \%$ & $27.7 \%$ & $36.7 \%$ & $23.5 \%$ & $\mathrm{~B}$ \\
\hline \multirow[t]{2}{*}{ Male $^{\text {B }}$} & 4,750 & 45.1 & $1.4 \%$ & $8.6 \%$ & $27.8 \%$ & $39.0 \%$ & $23.3 \%$ & A \\
\hline & $\begin{array}{l}\text { Number in } \\
\text { thousands }\end{array}$ & $\begin{array}{c}\text { \% of Total } \\
\text { Sample }\end{array}$ & $\begin{array}{c}<18.5 \\
\text { (Underweight) }\end{array}$ & $\begin{array}{c}20-24 \\
\text { (Normal) }\end{array}$ & $\begin{array}{c}\text { BMI } \\
25.0-29.9 \\
\text { (Overweight) }\end{array}$ & $\begin{array}{c}\text { 30-50 } \\
\text { (Obese) }\end{array}$ & & $\begin{array}{c}* \text { Outcome sig. } \\
\text { differences }\end{array}$ \\
\hline Total Adult Sample & 10,529 & 100.0 & $0.8 \%$ & $28.3 \%$ & $33.1 \%$ & $37.8 \%$ & & \\
\hline \multicolumn{9}{|l|}{ Sexual Identity } \\
\hline Heterosexual $^{A}$ & 9,942 & 94.4 & $0.8 \%$ & $28.2 \%$ & $33.3 \%$ & $37.7 \%$ & & \\
\hline Homosexual, gay, or lesbian ${ }^{B}$ & 246 & 2.3 & $0.4 \%$ & $32.9 \%$ & $30.5 \%$ & $36.2 \%$ & & \\
\hline Bisexual $^{\mathrm{C}}$ & 341 & 3.2 & $0.6 \%$ & $29.3 \%$ & $30.2 \%$ & $39.9 \%$ & & \\
\hline \multicolumn{9}{|l|}{ Sexual Behavior } \\
\hline No sexual experience with same-sex partner ${ }^{A}$ & 9,202 & 87.4 & $0.8 \%$ & $28.0 \%$ & $33.8 \%$ & $37.4 \%$ & & $\mathrm{~B}$ \\
\hline Sexual experience with same-sex partner $^{B}$ & 1,327 & 12.6 & $0.8 \%$ & $30.4 \%$ & $28.4 \%$ & $40.5 \%$ & & A \\
\hline \multicolumn{9}{|l|}{ Sexual Attraction } \\
\hline Only attracted to opposite $\operatorname{sex}^{\mathrm{A}}$ & 9,280 & 88.1 & $0.9 \%$ & $27.8 \%$ & $33.9 \%$ & $37.5 \%$ & & $\mathrm{~B}, \mathrm{C}$ \\
\hline Mostly attracted to opposite $\operatorname{sex}^{B}$ & 798 & 7.6 & $0.6 \%$ & $32.8 \%$ & $27.2 \%$ & $39.4 \%$ & & A \\
\hline Equally attracted to males \& females ${ }^{\mathrm{C}}$ & 187 & 1.8 & $1.1 \%$ & $31.0 \%$ & $21.9 \%$ & $46.0 \%$ & & $\mathrm{~A}, \mathrm{E}$ \\
\hline Mostly attracted to same $\operatorname{sex}^{\mathrm{D}}$ & 97 & 0.9 & $0.0 \%$ & $32.0 \%$ & $28.9 \%$ & $39.2 \%$ & & \\
\hline Only attracted to same $\operatorname{sex}^{\mathrm{E}}$ & 167 & 1.6 & $0.0 \%$ & $33.5 \%$ & $32.3 \%$ & $34.1 \%$ & & $\mathrm{C}$ \\
\hline \multicolumn{9}{|l|}{ Sex } \\
\hline Female $^{\mathrm{A}}$ & 5,779 & 54.9 & $1.0 \%$ & $32.5 \%$ & $26.6 \%$ & $40.0 \%$ & & $\mathrm{~B}$ \\
\hline \multirow[t]{3}{*}{ Male $^{B}$} & 4,750 & 45.1 & $0.7 \%$ & $23.3 \%$ & $41.0 \%$ & $35.0 \%$ & & $\mathrm{~A}$ \\
\hline & \multicolumn{7}{|c|}{ Access to Usual Source of Healthcare } & \\
\hline & $\begin{array}{l}\text { Number in } \\
\text { thousands }\end{array}$ & $\begin{array}{c}\text { \% of Total } \\
\text { Sample }\end{array}$ & Yes & No & & & & $\begin{array}{c}\text { *Outcome sig. } \\
\text { differences }\end{array}$ \\
\hline Total Adult Sample & 10,529 & 100.0 & $83.2 \%$ & $16.8 \%$ & & & & \\
\hline Sexual Identity & & & & & & & & \\
\hline Heterosexual $^{\mathrm{A}}$ & 9,942 & 94.4 & $83.0 \%$ & $17.0 \%$ & & & & \\
\hline Homosexual, gay, or lesbian'B & 246 & 2.3 & $87.0 \%$ & $13.0 \%$ & & & & \\
\hline Bisexual $^{\mathrm{C}}$ & 341 & 3.2 & $84.5 \%$ & $15.5 \%$ & & & & \\
\hline \multicolumn{9}{|l|}{ Sexual Behavior } \\
\hline No sexual experience with same-sex partner ${ }^{A}$ & 9,202 & 87.4 & $82.7 \%$ & $17.3 \%$ & & & & $\mathrm{~B}$ \\
\hline Sexual experience with same-sex partner ${ }^{B}$ & 1,327 & 12.6 & $86.6 \%$ & $13.4 \%$ & & & & A \\
\hline \multicolumn{9}{|l|}{ Sexual Attraction } \\
\hline Only attracted to opposite $\operatorname{sex}^{\mathrm{A}}$ & 9,280 & 88.1 & $82.7 \%$ & $17.3 \%$ & & & & $\mathrm{~B}$ \\
\hline Mostly attracted to opposite $\operatorname{sex}^{B}$ & 798 & 7.6 & $86.1 \%$ & $13.9 \%$ & & & & A \\
\hline Equally attracted to males $\&$ females ${ }^{\mathrm{C}}$ & 187 & 1.8 & $87.7 \%$ & $12.3 \%$ & & & & \\
\hline Mostly attracted to same $\operatorname{sex}^{\mathrm{D}}$ & 97 & 0.9 & $89.7 \%$ & $10.3 \%$ & & & & \\
\hline Only attracted to same $\operatorname{sex} x^{E}$ & 167 & 1.6 & $83.8 \%$ & $16.2 \%$ & & & & \\
\hline \multicolumn{9}{|l|}{ Sex } \\
\hline Female $^{\mathrm{A}}$ & 5,779 & 54.9 & $89.4 \%$ & $10.6 \%$ & & & & $\mathrm{~B}$ \\
\hline Male $^{B}$ & 4,750 & 45.1 & $75.6 \%$ & $24.4 \%$ & & & & A \\
\hline
\end{tabular}

Note. Table presents unweighted data analyses. Row totals may not equal 100.0 due to rounding.

This sample does not include pregnant females or those missing values listwise on these and other variables explored in later regression analyses.

*Items in same greyscale were grouped together in above Pearson $\chi^{2}$ tests due to small cell sizes and prior examples in the literature.

Superscripts denote a Pearson $\chi^{2}$ test of $p<0.05$ between that category and its corresponding superscript. Fisher's exact two-tailed test also run for $2 \times 2$ tables; results are consistent. 
TABLE 3-L. CH. 3 REGRESSIONS: IDENTITY: 35-44

Table 3-L. Regressions of Health Outcomes on Sexual Identity for Adults Aged 35-44 (N=10,529)

\begin{tabular}{|c|c|c|c|c|c|c|c|c|}
\hline & \multicolumn{2}{|c|}{ Self-Rated Health } & \multicolumn{2}{|c|}{ Overweight } & \multicolumn{2}{|c|}{ Obese } & \multicolumn{2}{|c|}{ Usual Source of Care } \\
\hline & OR & $95 \%$ CI & RRR & $95 \%$ CI & RRR & $95 \%$ CI & OR & $95 \% \mathrm{CI}$ \\
\hline Intercept & $7.31 * * *$ & $(4.30,12.43)$ & 0.80 & $(0.52,1.22)$ & 1.18 & $(0.83,1.68)$ & $9.87 * * *$ & $(6.21,15.70)$ \\
\hline \multicolumn{9}{|l|}{ Sexual Identity } \\
\hline Heterosexual (ref) & 1.0 & & 1.0 & & 1.0 & & 1.0 & \\
\hline Homosexual, gay, or lesbian & 0.86 & $(0.53,1.41)$ & 0.65 & $(0.41,1.02)$ & 0.90 & $(0.58,1.39)$ & 1.22 & $(0.56,2.64)$ \\
\hline Bisexual & 0.78 & $(0.50,1.19)$ & 0.99 & $(0.64,1.55)$ & 1.04 & $(0.70,1.56)$ & 1.07 & $(0.70,1.63)$ \\
\hline \multicolumn{9}{|l|}{ Survey Cycle } \\
\hline 2011-2013 (ref) & 1.0 & & 1.0 & & 1.0 & & 1.0 & \\
\hline 2013-2015 & $0.75^{*}$ & $(0.59,0.94)$ & $0.79 *$ & $(0.65,0.96)$ & 0.86 & $(0.70,1.06)$ & 1.01 & $(0.77,1.33)$ \\
\hline 2015-2017 & 0.81 & $(0.62,1.07)$ & 0.88 & $(0.70,1.09)$ & 0.87 & $(0.69,1.08)$ & 1.16 & $(0.88,1.52)$ \\
\hline 2017-2019 & $0.79 *$ & $(0.62,1.00)$ & 0.93 & $(0.74,1.16)$ & 1.07 & $(0.84,1.36)$ & $0.72 * *$ & $(0.56,0.92)$ \\
\hline \multicolumn{9}{|l|}{ Sex } \\
\hline Female (ref) & 1.0 & & 1.0 & & 1.0 & & 1.0 & \\
\hline Male & 1.03 & $(0.84,1.26)$ & $2.64 * * *$ & $(2.26,3.08)$ & $1.66 * * *$ & $(1.42,1.94)$ & $0.34 * * *$ & $(0.29,0.41)$ \\
\hline \multicolumn{9}{|l|}{ Race/ethnicity } \\
\hline White, non-Hispanic (ref) & 1.0 & & 1.0 & & 1.0 & & 1.0 & \\
\hline Hispanic & 0.78 & $(0.59,1.03)$ & $1.58^{* * *}$ & $(1.26,1.97)$ & $1.84 * * *$ & $(1.47,2.30)$ & 0.87 & $(0.68,1.10)$ \\
\hline Black, non-Hispanic & 1.07 & $(0.77,1.48)$ & $1.44 * *$ & $(1.13,1.82)$ & $2.54 * * *$ & $(2.02,3.18)$ & $1.33 *$ & $(1.00,1.78)$ \\
\hline Other, non-Hispanic & 0.73 & $(0.50,1.07)$ & $0.67 * *$ & $(0.53,0.85)$ & 0.88 & $(0.68,1.15)$ & 1.31 & $(0.98,1.75)$ \\
\hline \multicolumn{9}{|l|}{ Nativity } \\
\hline US-born (ref) & 1.0 & & 1.0 & & 1.0 & & 1.0 & \\
\hline Foreign-born & $1.56^{* *}$ & $(1.19,2.06)$ & $0.72 * *$ & $(0.57,0.89)$ & $0.42 * * *$ & $(0.33,0.53)$ & $0.74 * *$ & $(0.59,0.92)$ \\
\hline \multicolumn{9}{|l|}{ Residence } \\
\hline Principal MSA city (ref) & 1.0 & & 1.0 & & 1.0 & & 1.0 & \\
\hline Other MSA & 1.21 & $(0.99,1.48)$ & $1.19 *$ & $(1.03,1.38)$ & $1.30 * *$ & $(1.11,1.52)$ & 1.13 & $(0.95,1.35)$ \\
\hline Not MSA & 0.95 & $(0.72,1.25)$ & 1.11 & $(0.90,1.37)$ & $1.41 * *$ & $(1.11,1.80)$ & 1.28 & $(0.97,1.68)$ \\
\hline \multicolumn{9}{|l|}{ Education } \\
\hline Some high school or less (ref) & 1.0 & & 1.0 & & 1.0 & & 1.0 & \\
\hline High school diploma or GED & $1.88^{* * *}$ & $(1.44,2.45)$ & 1.01 & $(0.77,1.34)$ & 1.02 & $(0.78,1.34)$ & 1.04 & $(0.81,1.35)$ \\
\hline Some college & $1.63 * *$ & $(1.17,2.26)$ & 1.02 & $(0.75,1.37)$ & 1.02 & $(0.76,1.37)$ & 1.26 & $(0.93,1.70)$ \\
\hline College or graduate degree & $3.09 * * *$ & $(2.22,4.29)$ & $0.73 *$ & $(0.55,0.98)$ & $0.57 * * *$ & $(0.44,0.74)$ & 1.24 & $(0.95,1.62)$ \\
\hline \multicolumn{9}{|l|}{ Insurance } \\
\hline Private or Medi-Gap (ref) & 1.0 & & 1.0 & & 1.0 & & 1.0 & \\
\hline Medicaid, CHIP, State-sponsored & $0.47 * * *$ & $(0.35,0.63)$ & 1.05 & $(0.80,1.38)$ & 1.03 & $(0.79,1.33)$ & 0.91 & $(0.66,1.27)$ \\
\hline Medicare, military, government & $0.31^{* * *}$ & $(0.21,0.47)$ & 1.04 & $(0.77,1.41)$ & 0.89 & $(0.64,1.22)$ & 1.53 & $(0.97,2.42)$ \\
\hline Single-service, Indian Health Service, not covered & $0.65 * *$ & $(0.49,0.86)$ & 0.83 & $(0.65,1.05)$ & 0.84 & $(0.68,1.04)$ & $0.21 * * *$ & $(0.17,0.25)$ \\
\hline \multicolumn{9}{|l|}{ Household income, \% of federal poverty level } \\
\hline $0-99$ (ref) & 1.0 & & 1.0 & & 1.0 & & 1.0 & \\
\hline $100-299$ & $1.47 * *$ & $(1.16,1.87)$ & 1.10 & $(0.84,1.43)$ & 1.03 & $(0.82,1.30)$ & 1.00 & $(0.80,1.26)$ \\
\hline$>\mathbf{3 0 0} \%$ & $2.99 * * *$ & $(2.11,4.21)$ & 1.12 & $(0.84,1.49)$ & 0.88 & $(0.68,1.13)$ & $1.35^{*}$ & $(1.02,1.78)$ \\
\hline \multicolumn{9}{|l|}{ Access to Usual Source of Care } \\
\hline Yes (ref) & 1.0 & & 1.0 & & 1.0 & & & \\
\hline No & $0.66^{* *}$ & $(0.51,0.84)$ & 1.02 & $(0.84,1.24)$ & 0.82 & $(0.66,1.02)$ & & \\
\hline \multicolumn{9}{|l|}{ BMI } \\
\hline 18.5-24.9 (Normal) (ref) & 1.0 & & & & & & 1.0 & \\
\hline$<18.5$ (Underweight) & 0.88 & $(0.28,2.78)$ & & & & & 1.05 & $(0.44,2.51)$ \\
\hline 25.0-29.9 (Overweight) & 1.07 & $(0.82,1.39)$ & & & & & 0.96 & $(0.79,1.18)$ \\
\hline$>30.0$ (Obese) & $0.53 * * *$ & $(0.42,0.69)$ & & & & & 1.19 & $(0.95,1.48)$ \\
\hline
\end{tabular}

Note. Sample does not include pregnant females or those missing values listwise on these variables.

Self-rated general health $=0$ ) Poor, Fair 1) Good, Very good, Excellent. Logistic regression

The base outcome for the overweight and obese models is body mass index (BMI) that is low/normal ( $<25)$; overweight BMI is $25-29$, and obese BMI is $>30$. Multinomial regression.

Access to a usual source of care $=0$ ) No 1) Yes. Logistic regression.

$\mathrm{OR}=$ odds ratio $\mathrm{RRR}=$ relative risk ratio $\mathrm{CI}=$ confidence interval; $\mathrm{MSA}=$ metropolitan statistical area.

The $p$-values are determined by the "Pearson $\chi^{2}$ test with the Rao and Scott $(1981,1984)$ second-order correction" or the $F$ test (the default output from the Stata svy

tabulate command); ${ }^{*} p<0.05, * * p<0.01,{ }^{* * *} p<0.001$ 
TABLE 3-M. CH. 3 REGRESSIONS: ATTRACTION: 35-44

Table 3-M. Regressions of Health Outcomes on Sexual Attraction for Adults Aged 35-44 (N=10,529)

\begin{tabular}{|c|c|c|c|c|c|c|c|c|}
\hline & \multicolumn{2}{|c|}{ Self-Rated Health } & \multicolumn{2}{|c|}{ Overweight } & \multicolumn{2}{|c|}{ Obese } & \multicolumn{2}{|c|}{ Usual Source of Care } \\
\hline & OR & $95 \%$ CI & RRR & $95 \%$ CI & RRR & $95 \%$ CI & OR & $95 \%$ CI \\
\hline Intercept & $7.24 * * *$ & $(4.29,12.23)$ & 0.83 & $(0.55,1.27)$ & 1.20 & $(0.84,1.71)$ & $9.80 * * *$ & $(6.16,15.60)$ \\
\hline \multicolumn{9}{|l|}{ Sexual Attraction } \\
\hline Only attracted to opposite sex (ref) & 1.0 & & 1.0 & & 1.0 & & 1.0 & \\
\hline Mostly attracted to opposite sex & 1.03 & $(0.69,1.56)$ & $0.76^{*}$ & $(0.55,0.94)$ & 0.91 & $(0.70,1.19)$ & 1.02 & $(0.74,1.41)$ \\
\hline Equally attracted to males $\&$ females & 0.75 & $(0.45,1.26)$ & 0.59 & $(0.33,1.07)$ & 1.01 & $(0.61,1.67)$ & 1.62 & $(0.91,2.88)$ \\
\hline Mostly attracted to same sex & 0.90 & $(0.30,2.68)$ & 0.58 & $(0.28,1.21)$ & 0.69 & $(0.37,1.30)$ & 0.82 & $(0.19,3.43)$ \\
\hline Only attracted to same sex & 0.70 & $(0.40,1.21)$ & 0.70 & $(0.41,1.19)$ & 0.99 & $(0.58,1.69)$ & 1.53 & $(0.70,3.39)$ \\
\hline \multicolumn{9}{|l|}{ Survey Cycle } \\
\hline 2011-2013 (ref) & 1.0 & & 1.0 & & 1.0 & & 1.0 & \\
\hline 2013-2015 & $0.75^{*}$ & $(0.60,0.95)$ & $0.79 *$ & $(0.65,0.96)$ & 0.86 & $(0.70,1.06)$ & 1.01 & $(0.77,1.33)$ \\
\hline 2015-2017 & 0.81 & $(0.61,1.07)$ & 0.87 & $(0.70,1.09)$ & 0.87 & $(0.69,1.08)$ & 1.16 & $(0.88,1.52)$ \\
\hline 2017-2019 & $0.79 *$ & $(0.62,1.00)$ & 0.94 & $(0.75,1.17)$ & 1.07 & $(0.84,1.36)$ & $0.71 * *$ & $(0.56,0.91)$ \\
\hline \multicolumn{9}{|l|}{ Sex } \\
\hline Female (ref) & 1.0 & & 1.0 & & 1.0 & & 1.0 & \\
\hline Male & 1.03 & $(0.84,1.26)$ & $2.56 * * *$ & $(2.19,3.00)$ & $1.64 * * *$ & $(1.40,1.92)$ & $0.34 * * *$ & $(0.29,0.41)$ \\
\hline \multicolumn{9}{|l|}{ Race/ethnicity } \\
\hline White, non-Hispanic (ref) & 1.0 & & 1.0 & & 1.0 & & 1.0 & \\
\hline Hispanic & 0.78 & $(0.59,1.03)$ & $1.58 * * *$ & $(1.26,1.97)$ & $1.84 * * *$ & $(1.47,2.30)$ & 0.87 & $(0.68,1.10)$ \\
\hline Black, non-Hispanic & 1.07 & $(0.78,1.48)$ & $1.42 * *$ & $(1.12,1.79)$ & $2.53 * * *$ & $(2.01,3.17)$ & $1.34 *$ & $(1.00,1.78)$ \\
\hline Other, non-Hispanic & 0.73 & $(0.50,1.07)$ & $0.67 * *$ & $(0.53,0.86)$ & 0.89 & $(0.68,1.15)$ & 1.31 & $(0.98,1.76)$ \\
\hline \multicolumn{9}{|l|}{ Nativity } \\
\hline US-born (ref) & 1.0 & & 1.0 & & 1.0 & & 1.0 & \\
\hline Foreign-born & $1.57 * *$ & $(1.19,2.06)$ & $0.70 * *$ & $(0.56,0.88)$ & $0.41 * * *$ & $(0.33,0.52)$ & $0.74 * *$ & $(0.60,0.93)$ \\
\hline \multicolumn{9}{|l|}{ Residence } \\
\hline Principal MSA city (ref) & 1.0 & & 1.0 & & 1.0 & & 1.0 & \\
\hline Other MSA & 1.21 & $(0.99,1.48)$ & $1.18^{*}$ & $(1.02,1.37)$ & $1.30 * *$ & $(1.11,1.52)$ & 1.14 & $(0.96,1.35)$ \\
\hline Not MSA & 0.95 & $(0.72,1.25)$ & 1.09 & $(0.88,1.35)$ & $1.40 * *$ & $(1.10,1.78)$ & 1.28 & $(0.98,1.69)$ \\
\hline \multicolumn{9}{|l|}{ Education } \\
\hline Some high school or less (ref) & 1.0 & & 1.0 & & 1.0 & & 1.0 & \\
\hline High school diploma or GED & $1.88 * * *$ & $(1.44,2.46)$ & 1.02 & $(0.77,1.35)$ & 1.03 & $(0.78,1.34)$ & 1.04 & $(0.80,1.34)$ \\
\hline Some college & $1.63^{* *}$ & $(1.17,2.27)$ & 1.02 & $(0.76,1.38)$ & 1.02 & $(0.76,1.37)$ & 1.25 & $(0.93,1.69)$ \\
\hline College or graduate degree & $3.10 * * *$ & $(2.23,4.30)$ & $0.74 *$ & $(0.55,0.99)$ & $0.57 * * *$ & $(0.44,0.75)$ & 1.23 & $(0.94,1.61)$ \\
\hline \multicolumn{9}{|l|}{ Insurance } \\
\hline Private or Medi-Gap (ref) & 1.0 & & 1.0 & & 1.0 & & 1.0 & \\
\hline Medicaid, CHIP, State-sponsored & $0.47 * * *$ & $(0.35,0.63)$ & 1.06 & $(0.81,1.40)$ & 1.03 & $(0.80,1.33)$ & 0.91 & $(0.65,1.26)$ \\
\hline Medicare, military, government & $0.31 * * *$ & $(0.21,0.47)$ & 1.05 & $(0.78,1.41)$ & 0.89 & $(0.65,1.23)$ & 1.53 & $(0.97,2.43)$ \\
\hline Single-service, Indian Health Service, not covered & $0.65^{* *}$ & $(0.49,0.86)$ & 0.84 & $(0.66,1.06)$ & 0.84 & $(0.68,1.04)$ & $0.21 * * *$ & $(0.17,0.25)$ \\
\hline \multicolumn{9}{|l|}{ Household income, \% of federal poverty level } \\
\hline $0-99$ (ref) & 1.0 & & 1.0 & & 1.0 & & 1.0 & \\
\hline $100-299$ & $1.47 * *$ & $(1.16,1.87)$ & 1.10 & $(0.84,1.44)$ & 1.04 & $(0.83,1.30)$ & 1.01 & $(0.80,1.27)$ \\
\hline$>\mathbf{3 0 0} \%$ & $2.97 * * *$ & $(2.10,4.21)$ & 1.13 & $(0.85,1.50)$ & 0.88 & $(0.69,1.14)$ & $1.35^{*}$ & $(1.02,1.79)$ \\
\hline \multicolumn{9}{|l|}{ Access to Usual Source of Care } \\
\hline Yes (ref) & 1.0 & & 1.0 & & 1.0 & & & \\
\hline No & $0.66 * *$ & $(0.51,0.85)$ & 1.02 & $(0.84,1.24)$ & 0.82 & $(0.66,1.02)$ & & \\
\hline \multicolumn{9}{|l|}{ BMI } \\
\hline 18.5-24.9 (Normal) (ref) & 1.0 & & & & & & 1.0 & \\
\hline$<18.5$ (Underweight) & 0.88 & $(0.28,2.77)$ & & & & & 1.05 & $(0.44,2.52)$ \\
\hline 25.0-29.9 (Overweight) & 1.07 & $(0.82,1.39)$ & & & & & 0.97 & $(0.79,1.18)$ \\
\hline$>30.0$ (Obese) & $0.54 * * *$ & $(0.42,0.69)$ & & & & & 1.19 & $(0.95,1.48)$ \\
\hline
\end{tabular}

Note. Sample does not include pregnant females or those missing values listwise on these variables.

Self-rated general health $=0$ ) Poor, Fair 1) Good, Very good, Excellent. Logistic regression

The base outcome for the overweight and obese models is body mass index (BMI) that is low/normal $(<25)$; overweight BMI is $25-29$, and obese BMI is $>30$. Multinomial regression.

Access to a usual source of care $=0$ ) No 1) Yes. Logistic regression.

$\mathrm{OR}=$ odds ratio $\mathrm{RRR}=$ relative risk ratio $\mathrm{CI}=$ confidence interval; $\mathrm{MSA}=$ metropolitan statistical area.

The $p$-values are determined by the "Pearson $\chi^{2}$ test with the Rao and Scott $(1981,1984)$ second-order correction" or the $F$ test (the default output from the Stata svy

tabulate command); ${ }^{*} p<0.05, * * p<0.01, * * * p<0.001$ 
TABLE 3-N. CH. 3 REGRESSIONS: BEHAVIOR: 35-44

Table 3-N. Regressions of Health Outcomes on Sexual Behavior for Adults Aged 35-44 (N=10,529)

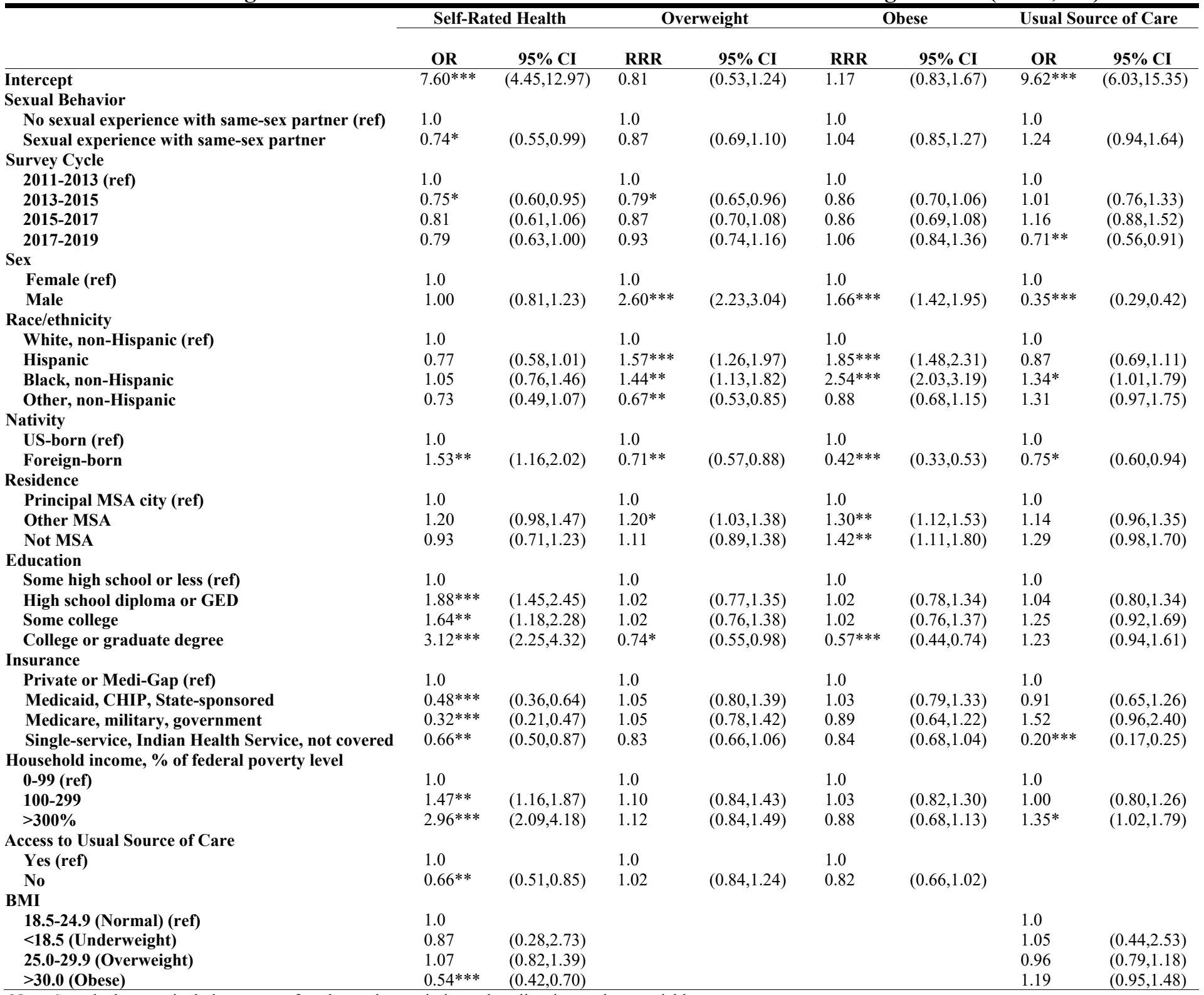

Note. Sample does not include pregnant females or those missing values listwise on these variables.

Self-rated general health $=0$ ) Poor, Fair 1) Good, Very good, Excellent. Logistic regression

The base outcome for the overweight and obese models is body mass index (BMI) that is low/normal (<25); overweight BMI is $25-29$, and obese BMI is $>30$. Multinomial regression.

Access to a usual source of care $=0$ ) No 1) Yes. Logistic regression.

$\mathrm{OR}=$ odds ratio $\mathrm{RRR}=$ relative risk ratio $\mathrm{CI}=$ confidence interval; $\mathrm{MSA}=$ metropolitan statistical area.

The $p$-values are determined by the "Pearson $\chi^{2}$ test with the Rao and Scott $(1981,1984)$ second-order correction" or the $F$ test (the default output from the Stata svy

tabulate command); ${ }^{*} p<0.05, * * p<0.01, * * * p<0.001$ 